\title{
TRANSCRANIAL DIRECT CURRENT STIMULATION AND THE THETA BETA RATIO IN THE CONTEXT OF ATTENTION DEFICIT HYPERACTIVITY DISORDER AND PEDIATRIC NEUROPSYCHIATRY
}

\author{
Derrick Matthew Buchanan
}

A dissertation submitted to the Faculty of Graduate and Postdoctoral Affairs in partial fulfillment of the requirements for the degree of

Doctor of Philosophy

In Neuroscience

Carleton University

Ottawa, ON, Canada

(C) 2020

DM Buchanan 


\begin{abstract}
Background: Attention deficit hyperactivity disorder (ADHD) is the most common neurodevelopmental condition worldwide. Transcranial direct current stimulation (tDCS) is a potential novel treatment option for ADHD. tDCS is widely used in adults, but disproportionately understudied in children. This thesis aimed to investigate the safety, tolerability, and acceptability of tDCS via a systematic review, a randomized, double-blind, placebo-controlled trial (RCT) in children and adults, and a qualitative acceptability study. Finally, tDCS treatment of ADHD could be optimized by using electroencephalography (EEG) and targeting a biomarker such as the theta-beta ratio (TBR). Since TBR remains controversial we aimed to test its validity. Methods: In the first study, a systematic review of tDCS safety in youth was conducted focusing on adverse effects and cognitive/neuroimaging/neuropsychiatric outcomes. In the second study, 60 participants (30 children aged 6-17 and 30 adults 18-45) received 10 minutes of $\mathrm{tDCS}$ twice, separated by a 1-hour interval. The stimulation amperage (sham, $0.5 \mathrm{~mA}, 1 \mathrm{~mA}$, and $2 \mathrm{~mA}$ ), and anode/cathode locations were randomized. The researcher conducting the outcome measures (at all 6 time points) and the participant were blinded to the amperage. In the third study, parents of 14 children who participated in the second study were interviewed regarding the acceptability/desirability/feasibility of tDCS compared to medication for their child. The fourth study used EEG and derived statistical and machine learning models to test the validity of TBR thresholds in a sample of healthy controls evaluated for ADHD ( $\mathrm{n}=189)$. Results: There were no serious adverse events reported across 1067 sessions in 156 children from 12 studies nor did any participants report any serious adverse effects in the randomized controlled trial. Moreover, children tolerated tDCS in a similar manner as adults. Parents find tDCS safe and acceptable and prefer it as an alternative to stimulant medication. The lack of side
\end{abstract}


effects was important for acceptability. TBR thresholds appear to be unrelated to ADHD.

Conclusion: tDCS is safe, tolerable, and acceptable to use in pediatric neuropsychiatry and there is a demand for clinically available tDCS especially in patients who are medication-resistant. The TBR threshold lacks clinical relevance and needs to be reconsidered in ADHD. 
This thesis is based upon the following four manuscripts:

Chapter 2 - Study 1: Buchanan, D.M.; Bogdanowiz, T.; Khanna, N.; Lockman-Dufour, G.; Robaey, P.; D'Angiulli, A. Systematic Review on the Safety and Tolerability of Transcranial Direct Current Stimulation in Children and Adolescents. In preparation for submission to Jama Pediatrics.

Chapter 3 - Study 2: Buchanan, D.M.; Amare, S.; Gaumon, G.; D'Angiulli, A.; Robaey, P. Safety and Tolerability of Transcranial Direct Current Stimulation in Children Adolescents and Adults: A Randomized Double-Blind Sham-Controlled Trial. In preparation for submission to the Journal of Child Psychology and Psychiatry and Allied Disciplines

Chapter 4 - Study 3: Buchanan, D.M.; D'Angiulli, A.; Samson, A.; Maisonneuve, A.R.; Robaey, P. Acceptability of transcranial direct current stimulation in children and adolescents with ADHD: The point of view of parents. J. Health Psychol. 2020, doi: $10.1177 / 1359105320937059$. This text is copied in full.

Chapter 5 - Study 4: Buchanan, D.M.; D'Angiulli, A.; Robaey, P. High and Low Clusters of Theta Beta Ratio have no Relationship with Attention Deficit Hyperactivity Disorder. In preparation for submission to Biological Psychiatry. 


\section{Other published works by Derrick Matthew Buchanan}

Buchanan, D.M.; Robaey, P.; D'Angiulli, A. (2020) What Do We Know about Transcranial Direct Current Stimulation for Major Depression? Brain Sciences. Vol. 10, 480. 2020. DOI:10.3390/brainsci10080480.

Buchanan, D.M.; Grant, J.; D'Angiulli A. (2019) Commercial Wireless vs. Standard Stationary EEG systems for Personalized Emotional Brain-Computer Interfaces: A Preliminary Reliability Check. Neuroscience Research Notes. 2(1): 7-15. DOI: 10.31117/neuroscirn.v2i1.21

Buchanan, D.M.; Croarkin, P.; Cohen Kadosh, R.; Kirton, A.; Masuda, F.; Noda, Y.; Gilbert, D.; Donaldson, P. (2019) Advances in Pediatric and Neurodevelopmental Brain Stimulation. International Brain Stimulation Conference Symposium. DOI: 10.13140/RG.2.2.12081.02408

Piexto, C.; Hyland, L.; Buchanan, D.M.; Langille, E.; Nahas, R. (2018) The Polytrauma Clinical Triad in Patients with Chronic Pain after Motor Vehicle Collision. Journal of Pain Research. 11:1927-1936. DOI: 10.2147/JPR.S165077

Buchanan, D.M.; Ros, T.; Nahas, R. (2020) Elevated and Slowed EEG Oscillations as a Signature of Post-Concussion Syndrome and Chronic Pain After Motor Vehicle Collision. Under review at Brain Injury.TBIN-2020-0292.

Buchanan, D.M. (2013) Sound Changes of the /h/-phoneme from Old English to Modern English. Canadian Undergraduate Journal of Cognitive Science; 47-54.

Buchanan, D.M; D’Angiulli, A.; Samson, A,; Robaey, P. (2019) Transcranial Direct Current Stimulation in Pediatric Neuropsychiatry: Safety Tolerability and Acceptability. The Journal of Psychiatry and Neuroscience.

Buchanan, D.M.; D'Angiulli, A.; Samson, A.; Amare, S.; Gaumond, G.; Robaey, P. (2019) Making transcranial direct current stimulation treatment in atypical child and adolescent neurodevelopment a reality: Translating safety tolerability and acceptability evidence from the laboratory into the doctor's office, the classroom, and home. Brain Stimulation. 12(2):474. DOI: $10.1016 /$ j.brs.2018.12.545 


\section{Co-Authors}

Amedeo D'Angiulli ${ }^{\mathrm{a}, \mathrm{b}}$

Philippe Robaey ${ }^{\mathrm{a}, \mathrm{c}, \mathrm{d}}$

Andreé Samson ${ }^{\mathrm{e}}$

Sarah Amare

Genvienve Gaumond ${ }^{c}$

Thomas Bogdanowicz ${ }^{\mathrm{a}, \mathrm{b}}$

Neha Khanna ${ }^{\mathrm{a}, \mathrm{b}}$

Guillaume Lockman-Dufour ${ }^{\mathrm{b}}$

Alexander R. Maisonneuve ${ }^{\mathrm{f}}$

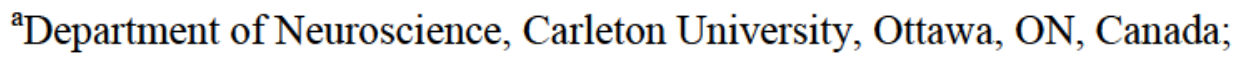

${ }^{\mathrm{b}}$ Neuroscience of Imagination Cognition Emotion Research Lab, Carleton University, Ottawa, ON, Canada;

'Neuropsychiatric Lab, Children's Hospital of Eastern Ontario, Ottawa, ON, Canada;

${ }^{\mathrm{d}}$ Department of Psychiatry, University of Ottawa, Ottawa, ON, Canada;

${ }^{\mathrm{e}}$ Faculty of Education, University of Ottawa, Ottawa, ON, Canada;

finterdisciplinary School of Health Sciences, University of Ottawa, Ottawa, ON, Canada. 


\section{Acknowledgements}

I would like to extend my most sincerest and grateful appreciation to my supervisor Dr. Amedeo D'Angiulli and co-supervisor Dr. Philippe Robaey. Each of you has provided me with incredible career changing opportunities and invaluable lessons about academia and neuroscience. $\mathrm{My} \mathrm{PhD}$ experience has been truly fantastic and I owe you each a debt of gratitude. I am also so grateful to all of my $\mathrm{PhD}$ committee members and other advisors in the department who were there for every major milestone. My sincerest thanks also goes out to the jurors of my defence and everyone else who has acted as a juror or referee for other applications of mine. I am also very thankful for each of my co-authors for their interest in collaboration, valuable contributions, and life lessons in the product of teamwork. I am eager to pay it forward with dividends. Finally I would like to thank my family and friends whose love and support have made my $\mathrm{PhD}$ journey the happiest and most enjoyable time of my life. I could write a second dissertation on how important you all are to me. I will summarize by saying you are all loved and I am grateful for you all. 


\section{Table of Contents}

\section{Dissertation}

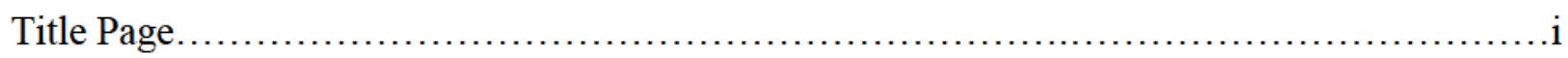

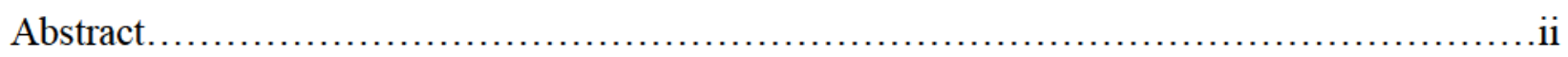

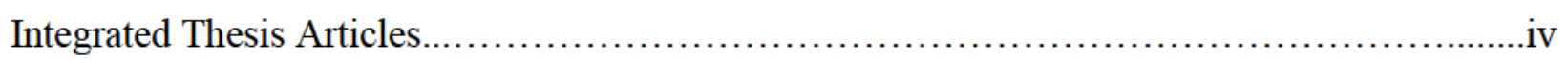

Other Published Articles by Derrick Matthew Buchanan........................................v

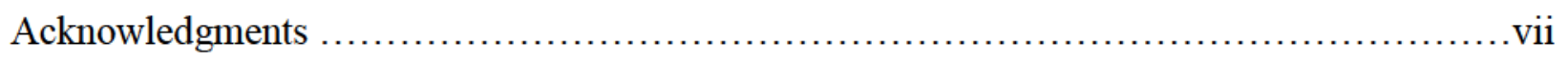

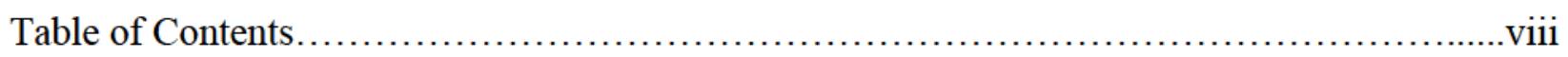

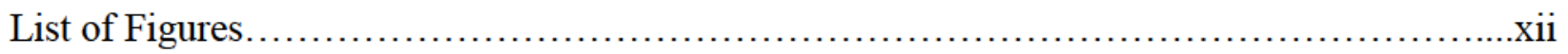

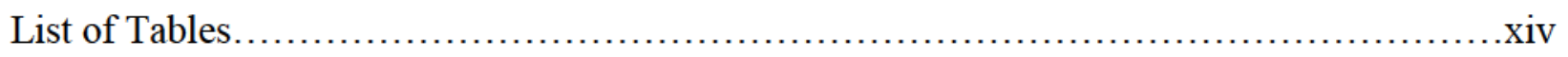

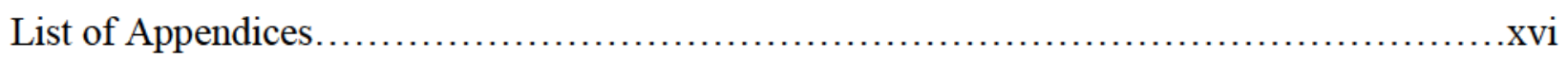

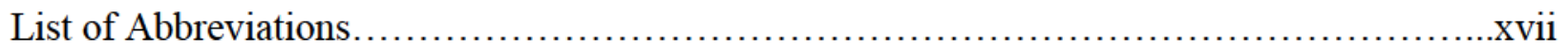

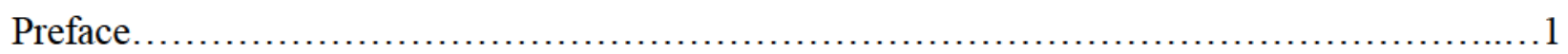

Chapter 1

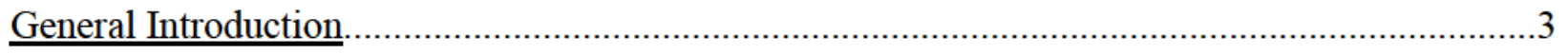

Chapter 2

Study One: Systematic Review on the Safety and Tolerability of Transcranial Direct Current

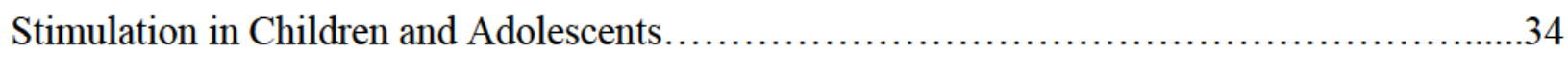

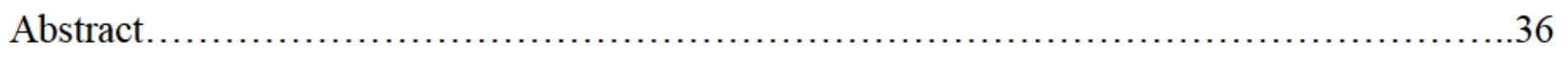

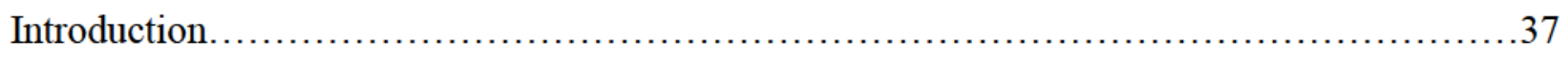

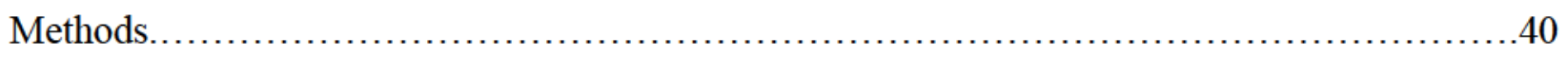

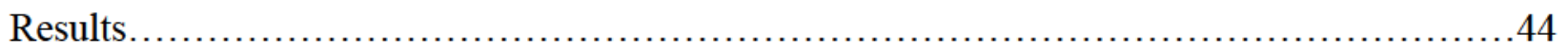




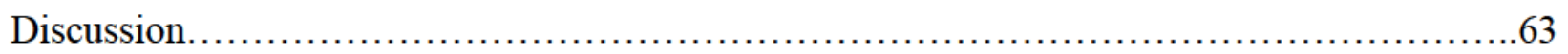

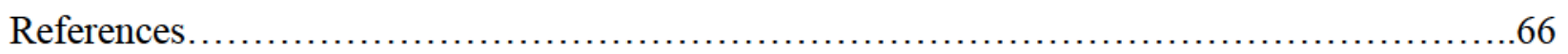

Chapter 3

Study Two: Safety and Tolerability of Transcranial Direct Current Stimulation in Children Adolescents and Adults: A Randomized Double-Blind Sham-Controlled Trial...................76

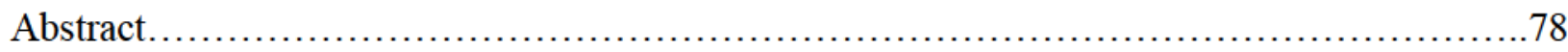

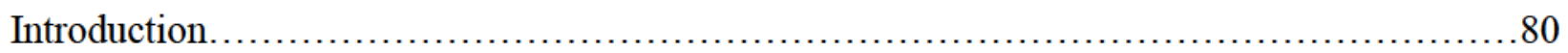

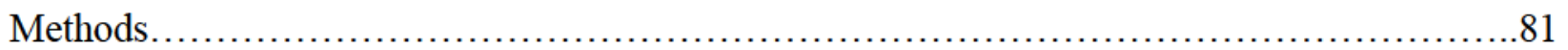

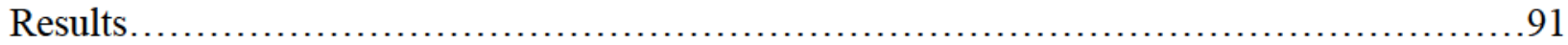

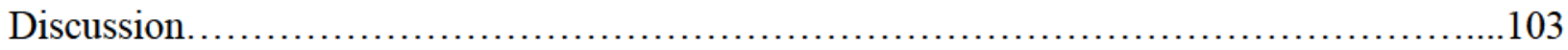

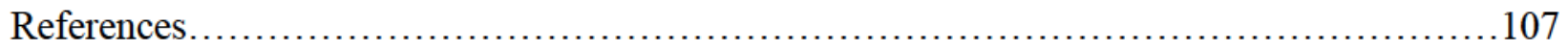

Chapter 4

Study Three: Acceptability of transcranial direct current stimulation in children and adolescents

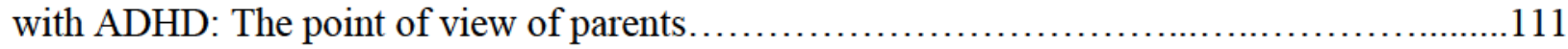

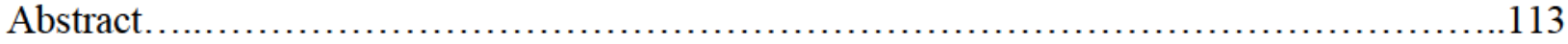

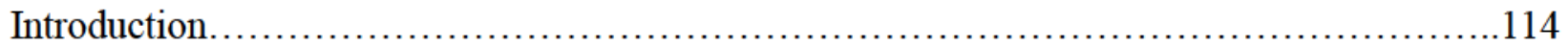

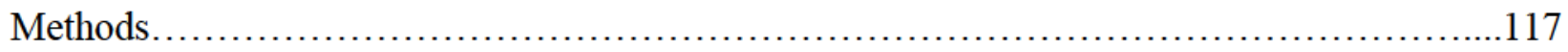

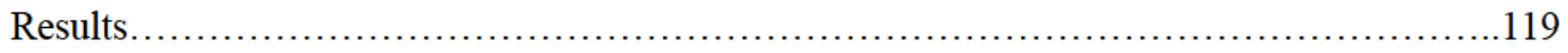

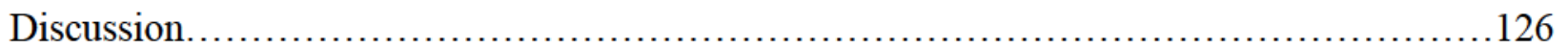

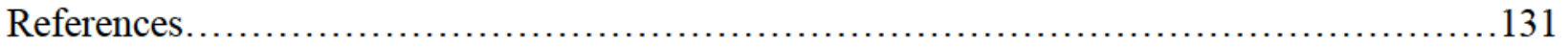




\section{Chapter 5}

Study Four: High and Low Clusters of Theta Beta Ratio have no Relationship with Attention

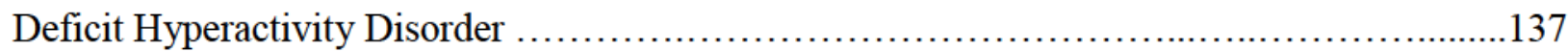

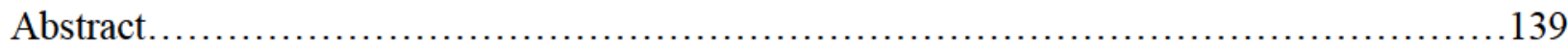

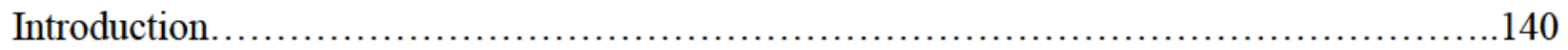

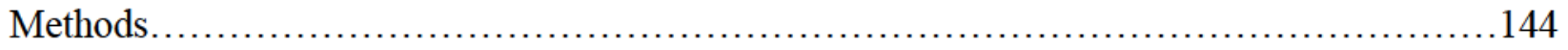

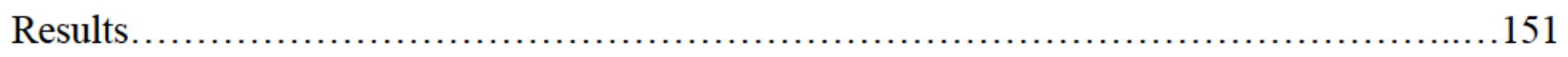

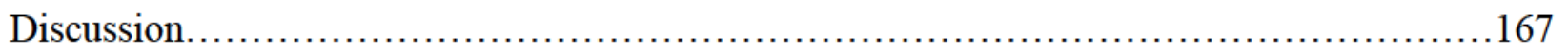

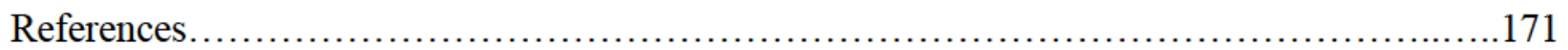

Chapter 6

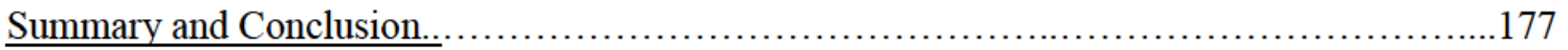

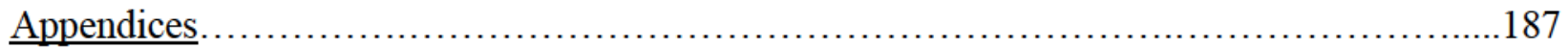

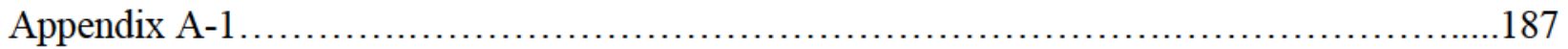

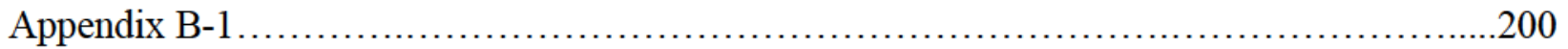

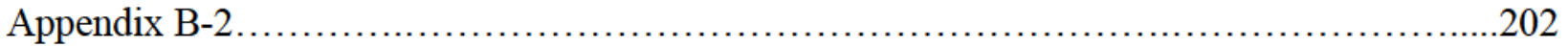

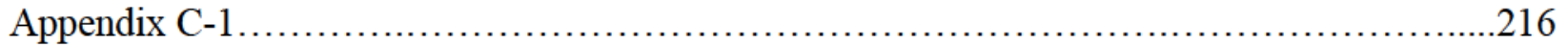

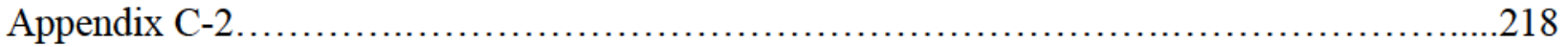

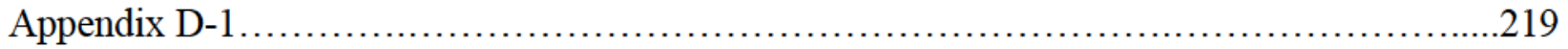


Appendix D-2.

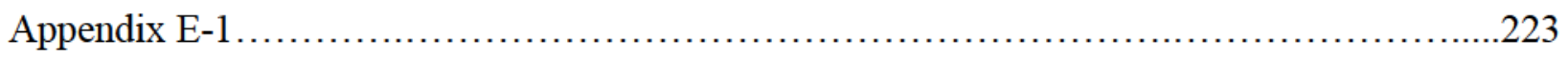

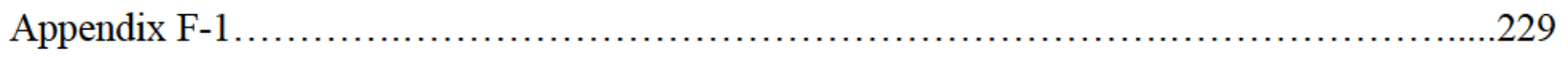




\section{List of Figures}

\section{Chapter 1}

Figure 1 - Research Program Progression

Figure 2 - Proposed Mechanisms of tDCS

\section{Chapter 2}

Figure 1 - Literature search PRISMA flow diagram

Figure 2 - Risk of bias assessment of each included study

\section{Chapter 3}

Figure 1 - 3D bar graph of side effects at each time point for children and adults

Figure 2 - 3D bar graph of side effects at each time point for females and males

Figure 3 - 3D bar graph of side effects at each time point for clinical and controls

Figure 4 - Boxplot of Bayesian factor average for age sex and clinical status

\section{Chapter 4}

This chapter has no figures

\section{Chapter 5}

Figure 1a - Histogram of subjects in the high and low TBR clusters

Figure 1b - Overlay of the high and low TBR cluster distributions

Figure 1c - Histogram of subjects in the high and low TBR clusters adopted from Bussalb for comparison. Note: their values should be squared for comparison.

Figure 1d - Overlay of the high and low TBR cluster distributions adopted from Bussalb for comparison. Note: their values should be squared for comparison.

Figure 1e - Boxplot of high and low TBR clusters averaged at $\mathrm{Fz}$ and $\mathrm{Cz}$ 
Figure 2a - Population pyramid count of inattentive ADHD symptoms in subjects from the high and low TBR clusters

Figure 2b - Population pyramid count of hyperactive ADHD symptoms in subjects from the high and low TBR clusters

Figure 2c - Population pyramid count of overall ADHD symptoms in subjects from the high and low TBR clusters

Figure 3a - Boxplot for inattention continuous measurement in the high and low TBR clusters

Figure 3b - Boxplot for hyperactive continuous measurement in the high and low TBR clusters

Figure 3c - Boxplot for overall ADHD continuous measurement in the high and low TBR clusters

Figure 4 - Boxplot of male and female TBR averaged at Fz and $\mathrm{Cz}$

Figure 5a - Boxplot for inattention continuous measurement in males and females

Figure 5b - Boxplot for hyperactive continuous measurement in males and females

Figure 5c - Boxplot for overall ADHD continuous measurement in males and females

\section{Chapter 6}

This chapter has no figures 


\section{List of Tables}

\section{Chapter 1}

This chapter has no tables.

\section{Chapter 2}

Table 1 - Table of common, uncommon, and rare side effects previously investigated in tDCS

Table 2 - tDCS exposure for age and population

Table 3 - Author demographic and materials used

Table 4 - Adverse events reports

\section{Chapter 3}

Table 1 - Distribution of participants across different conditions of amperages as a function of age, sex and clinical status. The average amplitude is presented in the last column.

Table 2 - Distribution of participants across different electrode locations as a function of age, sex and clinical status

Table 3 - Distribution of participants across different amperages as a function of age, sex and clinical status

Table 4 - F statistics of each group effect and associated partial $\eta$, as well as Bayes factor

Table 5 - Effect of Low vs. High amperage across the whole sample, and interactions with age, sex and clinical status

\section{Chapter 4}

This chapter has no tables

\section{Chapter 5}

Table 1 - Symptoms of ADHD categorized by two different symptomologies: Inattention and Hyperactivity/Impulsivity 
Table 2 - ADHD Diagnostic Criteria by DSM-IV-TR

Table 3 - TBR thresholds from the literature compared to the present sample

Table 4 - Chi-square comparisons for 1-4+ inattentive, hyperactive, and overall ADHD symptoms between high and low TBR cluster

Table 5 - Symptom counts for inattentive, hyperactive, and overall ADHD symptoms

Table 6 - T-tests and Bayes comparisons for averaged continuous inattentive, hyperactive, and overall ADHD scores for clusters

Table 7 - Chi-square comparisons for 1-4+ inattentive, hyperactive, and overall ADHD symptoms between males and females

Table 8 - T-tests and Bayes comparisons for averaged continuous inattentive, hyperactive, and overall ADHD scores for sex

\section{Chapter 6}

This chapter has no tables 


\section{List of Appendices}

Appendix A-1 - Study 1 supplementary data

Appendix B-1 - Study 2 supplementary data

Appendix B-2 - Study 2 data collection forms

Appendix C-1 - Study 3 data collection forms

Appendix C-2 - Study 3 Theoretical Framework of Acceptability and tDCS

Appendix D-1 - Study 4 supplementary data

Appendix D-2 - Study 4 EEG acquisition set up and testing battery

Appendix E-1 - Publication by DMB - What do we know about transcranial direct current stimulation for Major Depression.

Appendix F-1 - Publication by DMB - Commercial wireless versus standard stationary EEG systems for personalized emotional brain-computer interfaces: a preliminary reliability check. 


\section{List of Abbreviations}

ADHD - attention deficit hyperactivity disorder

$\mathrm{AE}$ - adverse event

AHA - assisting hand assessment

AMPA - $\alpha$-amino-3-hydroxy-5-methyl-4-isoxazolepropionic acid

ANCOVA - analysis of covariance

ANOVA - analysis of variance

AS - Asperger syndrome

ASD - autism spectrum disorder

CANTAB - Cambridge neuropsychological test automated battery

CHEO - Children's Hospital of Eastern Ontario

COS - childhood onset schizophrenia

$\mathrm{CP}$ - cerebral palsy

CSD - current source density

CSWS - continuous spikes and waves during sleep

CVLT - California verbal learning test

$\mathrm{Cz}$ - electrode placed over the sensory motor area

DA - dopamine

DAT - dopamine transporter

DLPFC - dorsolateral prefrontal cortex

DSM-IV-TR - diagnostical statistical manual version IV

EEG - electroencephalogram

EKG - electrocardiography 
ELD - expressive language disorder;

EMG - electromyography

EOG - electrooculography

ERP - event related potentials

$\mathrm{Fz}$ - electrode placed over the middle of the frontal cortex

GP - global dyspraxia

HC - healthy control

HD - high definition

IAF - individualized alpha frequency

IFG - inferior frontal gyrus

iTBS - intermittent theta burst stimulation (type of TMS)

LKS - Landau-Kleffner syndrome

LMIFG - left medial inferior frontal gyrus

M1 - primary motor cortex

MANOVA - multivariate analysis of variance

MEP - motor evoked response

MMSE - mini mental status examination

MRI - magnetic resonance imaging

NA - norepinephrine

NBT - neurophysiological biomarker toolbox

NFB - neurofeedback

$\mathrm{NNH}$ - number needed to harm

OFC - orbitofrontal gyrus 
PDD-NOS - pervasive developmental disorder not otherwise specified

PET - positron emission tomography

PRISMA - preferred reporting in systematic reviews and meta-analyses

PVL - periventricular leukomalacia

qEEG - quantitative electroencephalography

QOL - quality of life

$\mathrm{RCT}$ - randomized controlled trial

REB - research ethics board

rTMS - repetitive transcranial magnetic stimulation (implies repeated pulses)

STG - superior temporal gyrus

SVM - support vector machine

TBR - theta beta ratio

tDCS - transcranial direct current stimulation

TFA - theoretical framework of acceptability

TMS - transcranial magnetic stimulation (implies single pulse)

$\mathrm{UCP}$ - unilateral cerebral palsy 


\section{Preface}

Supervisor statement: In this integrated thesis Derrick Matthew Buchanan (DMB) was the lead author of each article and contributed substantially to this overall program of research. DMB contributed to each of these articles throughout the entire research process from inception to publication. With the help of his supervisors he personally designed each study and wrote the corresponding protocols, consent forms, data collection forms, etc. DMB also managed all aspects of our cross appointed research ethics board submissions (Carleton and CHEO) and personally coordinated recruitment of participants, scheduling of participants, and every aspect of data collection. DMB was also in charge of handling an internal quality assurance audit at CHEO. Moreover, DMB was the leader for each study on data pre-processing, processing, and analysis. With the help of his supervisors he personally managed multiple research assistants who contributed to data collection. Five undergraduate research assistants that contributed to data collection and processing have been included as co-authors across different manuscripts in this thesis. Thomas Bogdanowicz, Neha Khanna, and Guillaume Lockman-Dufour contributed the data processing in Study 1. Their role was to help extract data from papers that were being included in our systematic review. The data extraction was validated by DMB and disputes were settled by Amedeo D’Angiulli (AD). Sarah Amare (SA) and Genvienve Gaumond (GG) contributed to data collection and pre-processing in Study 2. Since one researcher was blinded during our randomized controlled trial another had to be present during each session to collect questionnaire data from the participants, as well as the baseline and follow up outcome measures. SA also contributed to data processing in Study 3 by means of interview transcription. Transcripts were further validated and completed by DMB. In Study 3 DMB and his supervisors designed a qualitative research study and therefore pursued collaboration with Andre Samson (AS), an expert in the field. AS contributed to the conceptual and methodological interpretation 
of the data in this study and provided revisions for the manuscript. Alexandre Maisonneuve, A $\mathrm{PhD}$ student of AS, contributed as a research assistant by coding the themes that emerged from the qualitative interviews. PR was responsible for all of the EEG data acquisition that was part of the Quebec New Born Twin database used in Study 4. DMBs role in Study 4 involved all of the EEG pre-processing, processing, and analysis. This included creating, contributing, and merging, an entire EEG biomarker database with the behavioural and psychological data collected during the Quebec New Born Twin study. DMB also conducted all of the statistical and machine learning analyses, and wrote the manuscript. $\mathrm{AD}$ and $\mathrm{PR}$ share senior authorship across these manuscripts. Both $\mathrm{AD}$ and PR contributed substantially to each manuscript: by providing expertise, conceptual design input, generating hypotheses, generating resources, monitoring statistical analyses, critical feedback, manuscript revisions, and ultimate oversight of the included articles, research design, and research staff. During his $\mathrm{PhD}$ DMB also: taught 4 undergraduate courses in the Department of Neuroscience focused on clinical neuroscience and neuropsychiatry, presented at dozens of conferences, completed 16 TAships, a semester in the Neurology Imaging Cognition Lab in Geneva, a certificate in Transcranial direct current stimulation for neuropsychiatry from Harvard Medical School, and a certificate for Positron Emission Tomography from McGill Montreal Neurological Institute.
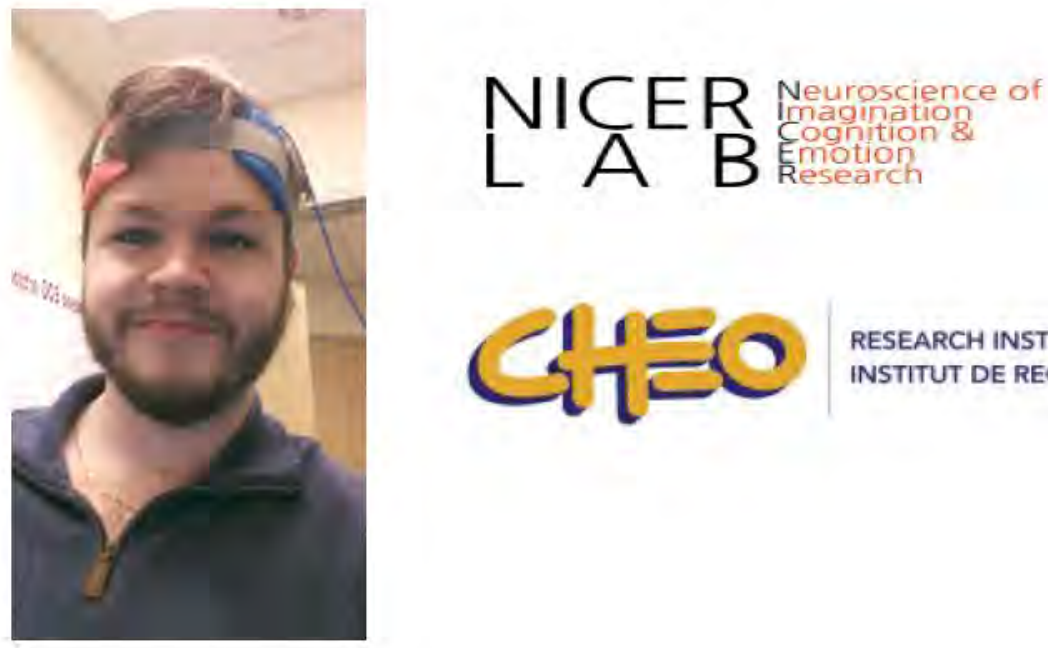

RESEARCH INSTITUTE INSTITUT DE RECHERCHE

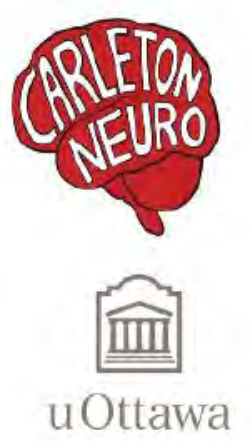




\section{Chapter 1:}

General Introduction 


\section{General Introduction}

The objective of this thesis is to investigate the safety, tolerability, and acceptability of transcranial direct current stimulation and the validity of the theta beta ratio signature in the context of pediatric attention deficit hyperactivity disorder. This introduction will outline pertinent literature that has shaped the field of pediatric tDCS while highlighting the specific contributions made by the present program of research.

Attention deficit hyperactivity disorder (ADHD) is the most common neurodevelopmental condition affecting $5 \%$ of children worldwide [1,2]. Current treatment methods are generally limited to long term commitments to pharmaceuticals, behavioural or psychosocial therapies, or neurofeedback (NFB) which are not always effective or manageable for parents and children. Currently there is no intensive short term long lasting treatment option for ADHD [3,4]. Transcranial direct current stimulation (tDCS) is a novel potential treatment option for children with ADHD [5-8] and other neuropsychiatric conditions $[9,10]$ that may be more manageable and less of a risk than medication [11]. tDCS is a portable non-invasive neuromodulation technique that utilizes low amplitude $(0-2 \mathrm{~mA})$ direct currents applied via scalp electrodes to modulate the level of excitability in specific cortical regions over the course of typically $10-20$ sessions (2-5x weekly) [12]. The potential for short term intensive tDCS interventions to provide long lasting remission in $\mathrm{ADHD}$ and other neuropsychiatric conditions is what makes it worthy of investigation.

The interest around tDCS is driven by its potential to induce long lasting neuroplastic changes to aid in the treatment or management of disorders like ADHD [8], major depression [13], memory deficits and motor control in Parkinson's [14], memory deterioration in Alzheimer's dementia [15], reduction of pain in chronic pain conditions [16], and expediting 
stroke recovery [17]. There are now thousands of publications for tDCS use in adults and this has led to a growing interest in its applications for children [10,18]. Rivera-Urbina et al. [10] highlighted evidence for pediatric tDCS in epilepsy, autism, dyslexia, ADHD, and other psychiatric disorders like depression and schizophrenia. Despite the growing evidence and interest, there remains a degree of uncertainty surrounding tDCS use in children. Such reservations are primarily centered on the current lack of evidence for the short and long term safety and tolerability of tDCS in the developing brain.

Presently, tDCS is considered to be safe, tolerable, and acceptable to use in adults [1825] however it is significantly under researched in pediatrics [26]. In 2016 Bikson and colleagues [23] published an evidenced based update on tDCS safety noting that only $5 \%$ of all tDCS studies were conducted in children and adolescents. Within this narrow $5 \%$ margin only a handful of studies actually measured or assessed safety and tolerability [26-35]. This disproportionately low representation of children in the literature may be partially due to the scarcity of available safety evidence. It is critical to firmly establish the safety and tolerability of tDCS in pediatrics in order to move forward with more efficacy trials. Since $95 \%$ of the tDCS literature has been conducted in adults, it would be advantageous to utilize a translational paradigm that compares the safety and tolerability of tDCS in adults with children. Effectively, this would facilitate the translation of efficacious adult protocols into pediatrics. This thesis contributed several articles to fill this gap.

\section{The Present Thesis}

1) A systematic review of the literature including all of the published studies that have objectively evaluated tDCS safety and tolerability in pediatrics. The aim of this study was to 
establish a firm foundation of tDCS safety in pediatrics to facilitate knowledge transfer to researchers and clinicians, and to uncover the strengths and limitations of the current safety evidence. 2) A randomized double-blind sham-controlled safety trial of tDCS in children, adolescents, and adults. The aim of this study was to directly compare safety and tolerability of tDCS between children and adults in order to facilitate the transferability of adult protocols to pediatrics. 3) A qualitative acceptability study on the use of tDCS in children with ADHD. The aim of this study was to facilitate the practical translation of tDCS by listening to parents' points of view and decision making process surrounding using tDCS as a treatment in their child. 4) An ADHD biomarker study in children. The aim of this study was to investigate the validity of a potential electrophysiological biomarker called the theta beta ratio (TBR) in ADHD.

Figure 1. Research Program Progression

Study 1

Systematic Review of

Safety, Tolerability of

tDCS in Youth
Study 2

RCT Safety/

Tolerability tDCS

Study in healthy and

ADHD youth
Study 4

Validating

Follow up tDCS

Acceptability Study

on Study 2 electrophysiological

biomarkers for

ADHD

neuromodulation 
In the first study, we conducted a systematic review of tDCS safety studies in children and adolescents. Since the tDCS safety literature is largely limited to reports of adverse events, it was beneficial to conduct a systematic review that aggregated objective measures of tDCS safety. In our systematic review safety studies were defined as studies that assessed pathological changes in physiology, and other objective medical evaluations like change in performance in a given physical, cognitive, or psychological domain as primary outcomes. The primary outcomes of this study were in line with previous adult safety and tolerability reviews [20,21,23]: tolerability was measured via rate and severity of side effects/adverse events (AEs), and acceptability via rate of dropouts. We hypothesized that there would be a positive consensus between the investigations such that tDCS is safe and tolerable in children and adolescents.

Our second study prospectively examined children and adults from healthy and clinical samples in a randomized double-blind sham-controlled safety protocol. Children and adults were assigned to multiple sessions of tDCS with randomized amperage and electrode locations based on common adult protocols [20]. Since tDCS is very well evidenced in adults, use of a protocol that directly compared adult and pediatric safety was beneficial to the translation of common adult protocols to youth. The primary outcomes of this study were safety (adverse events), tolerability (side effects), and acceptability (drop outs); where the operationalization of these variables can be found in previous tDCS safety literature [23]. We hypothesized that tDCS would be safe, tolerable, and acceptable and that there would be no differences in safety or tolerability between children and adults. We further hypothesized that there would be no differences between males vs. females, or clinical vs. healthy samples. Through this design and the use of Bayesian statistics [36-38] we analyzed and determined if children and adults experienced the same frequency, intensity, and type of side effects from tDCS. 
The third study addressed tDCS acceptability by interviewing families of the children who participated in the randomized controlled safety study (Study 2). Since Study 1 and Study 2 have confirmed the general safety and tolerability of tDCS in youth, it was prudent to next evaluate whether or not this treatment is even acceptable among parents for treating ADHD. Here we gained a deeper understanding of families' experiences of tDCS use in their child and what makes it acceptable compared to medication. Given the subjective nature of each family's experience it was advantageous to utilize a semi-structured interview approach $[39,40]$. The primary outcome of this study was a theory of what makes tDCS acceptable; based on the themes that emerged from parental interviews (e.g., concerns for safety of tDCS, cost, insurance coverage, side effects vs. medication, efficacy vs. medication). We hypothesized that tDCS would be acceptable in pediatric ADHD.

The fourth and final study aimed to investigate the validity of the theta beta ratio as an electrophysiological marker such that it may be helpful in the characterization of ADHD and a useful target for tDCS. This study replicated the analysis of a recently published study from Bussalb et al. [41] who found that patients with ADHD could be statistically sorted into groups with very high theta beta ratio (TBR) and those with normal TBR. To that end, Bussalb suggested that ADHD patients falling above the threshold, putting them in the high TBR category, would be good candidates for neuromodulation treatment. Since high TBR is alleged to be a marker of ADHD, it should have little to no prevalence in a healthy sample. Therefore, to test the validity of TBR we replicated the analysis of Bussalb et al., [41] using a healthy sample of age controlled children; vs. Bussalb who used a sample of adults and children with ADHD. If elevated TBR is a good marker for ADHD, we should not find this marker in healthy children. The primary outcome of this study was the high and low TBR clusters that we derived in our 
healthy sample. Our secondary outcomes involved testing the relationship between the TBR threshold and ADHD symptoms among otherwise typically developing children. We hypothesized that even a normative sample of healthy controls would exhibit a high and low cluster of TBR and that these clusters would not be related to ADHD symptomology. The clinical implication of this study is such that TBR thresholds are irrelevant for prescribing ADHD treatments.

\section{tDCS in ADHD}

The heterogeneous nature of ADHD psychology makes elucidating a clear neurobiological mechanism a challenging research question; but for the same reasons an important one. This is further complicated by the fact that typically developing children also exhibit a wide range of ADHD symptoms making it more difficult to parse out abnormalities between patients and controls. The literature is still lacking a clear understanding of ADHD neurobiology. In this section I will explain some of the leading neurobiological theories of $\mathrm{ADHD}$ from the top down. There are multiple approaches that aim to characterize ADHD, but so far the behavioural psychology approach is the only one used for diagnostic purposes [42]. To that end, researchers have aimed to understand how specific psychological symptom profiles (sub-types) $[43,44]$ associate with neurobiological mechanisms of ADHD such as anatomical [45-47], functional [48-52], or neurochemical markers [53-55].

There are a few leading theories that each characterize ADHD from a different level. From a top down point of view most of what we know about ADHD is at the top level and this is based on the psychological diagnostic criteria and behavioural / cognitive dysfunctions associated with ADHD [42]. For instance, children with ADHD have increased impulsiveness 
and decreased attention and these symptoms are clinically relevant to prescribing ADHD treatments. Next is the cortical network level known as the prefrontal hypothesis of ADHD [46,47]. The cortical level attempts to explain ADHD symptomology by examining neuroanatomy and its corresponding function. In the case of ADHD, the neuropathological regions tend to include hypoactive dorsolateral prefrontal cortex (DLPFC), orbitofrontal cortex (OFC), and right inferior frontal gyrus (rIFG). The DLPFC for example is implicated in impulse control and working memory and therefore increasing the activity of DLPFC should subsequently decrease impulsivity and increase working memory. Down another level, these regions may be further characterized by measuring the electrophysiological activity within the cortex using electroencephalography (EEG) [41,56-60]. The sum of excitatory and inhibitory post synaptic potentials can be monitored via EEG and enable a more dynamic understanding of the cortex. In ADHD one of the prevailing hypotheses based on EEG oscillations is the TBR hypothesis $[41,44,57,61-66]$. The TBR hypothesis aims to characterize ADHD using electrophysiological oscillations emitting from the cortex at sites $\mathrm{Fz}$ and $\mathrm{Cz}$ in the theta $(4-8 \mathrm{~Hz})$ and beta $(12-30 \mathrm{~Hz})$ frequency range. The TBR hypothesis posits that patient's with ADHD have elevated theta band activity in ratio to beta band activity. TDCS may be able to modulate these oscillations and decrease theta activity. Finally, the lowest level is neurochemical [53-55]. The neurochemical hypothesis attempts to characterize ADHD through the identification of changes in neurotransmitter systems. Patients with ADHD are proposed to have irregular monoamine reward circuitry; in turn, tDCS has been shown to promote the release of dopamine [67]. Therefore, tDCS may increase the amount of dopamine available in the synapse through mechanisms like increased DA transporter (DATs) or DA1 and DA2 receptors $[55,68]$. 
Putting it all together from the top down: tDCS can be applied to scalp regions that correspond to cortical sites such as DLPFC. The positive electrical current will flow from the scalp to the cortex via the apical dendrites of pyramidal neurons $[69,70]$. As the amount of positive electrical activity in a given cortical region increases the sum of excitatory post synaptic potentials will become strong enough to depolarize the soma of neurons [71] leading to action potentials and the release of neurotransmitters. Evidence from tDCS animal studies has shown that the propagation of excitatory post synaptic potentials and release of neurotransmitters might have different effects / mechanisms such as: the phosphorylation and translocation of $\alpha$-amino-3hydroxy-5-methyl-4-isoxazolepropionic acid (AMPA) receptors in the hypothalamus and hippocampus [72], and the migration of axon terminal or dendritic spines [73]. A recent human study using positron emission tomography (PET) also revealed that anodal tDCS over the LDLPFC, the target for ADHD, leads to the release of dopamine in the right ventral striatum and this was significantly correlated with subjects' accuracy on the Cambridge Neuropsychological Test Automated Battery (CANTAB) [67]. In summary, the successful modulation of these cortical regions and neurotransmitter systems in ADHD should be accompanied by a subsequent reduction in ADHD symptomology. Theoretically, this is how tDCS is expected to treat ADHD (Figure 2).

In many ways tDCS is similar and often interchangeable with another kindred brain stimulation technique: transcranial magnetic stimulation. The theories, implications, and applications for tDCS and TMS are highly congruent across the different levels in Figure 2 [7482]. Moreover, the treatment protocols for tDCS and TMS are highly similar, both regularly targeting the same cortical sites for the same conditions, and both requiring daily sessions, for around 4 weeks to achieve long lasting treatment effects. The main distinction is that tDCS only 
modulates the likelihood of action potentials whereas TMS uses strong magnetic pulses to rapidly change the polarity of neurons causing action potentials in a specific cortical region [83]. In this way the immediate effects of TMS also tend to be more focalized whereas the immediate effects of tDCS are more diffuse. For these reasons, the literature often describes tDCS as a modulation technique and TMS as a stimulation technique.

Within the TMS family there is also repetitive TMS (rTMS) and intermittent theta burst TMS (iTBS). While TMS is useful in preclinical cognitive neuroscience experiments rTMS and iTBS are the tools used in clinical trials and real world treatments. rTMS and iTBS work by the same mechanistic principle as TMS; using a magnetic current to rapidly change the polarity of neurons. Also, rTMS and iTBS both pulse the cortex hundreds of times in a given treatment session. The difference between rTMS and iTBS is that conventional rTMS is delivered at $10 \mathrm{~Hz}$ for 37.5 minutes, whereas iTBS is delivered at $50 \mathrm{~Hz}$ for 3 minutes [84]. Although TMS seems to work similarly at the same levels as tDCS there are some distinct practical advantages of tDCS such as its portability, low cost, and the device is simple to operate. Future research will have to involve non-inferiority trials of tDCS and rTMS/iTBS in order to elucidate if either has superior efficacy. In the next paragraphs we will explore the current literature on tDCS in ADHD. 
Figure 2. Proposed Mechanisms of tDCS

\section{Levels of tDCS Mechanisms of Action in ADHD}

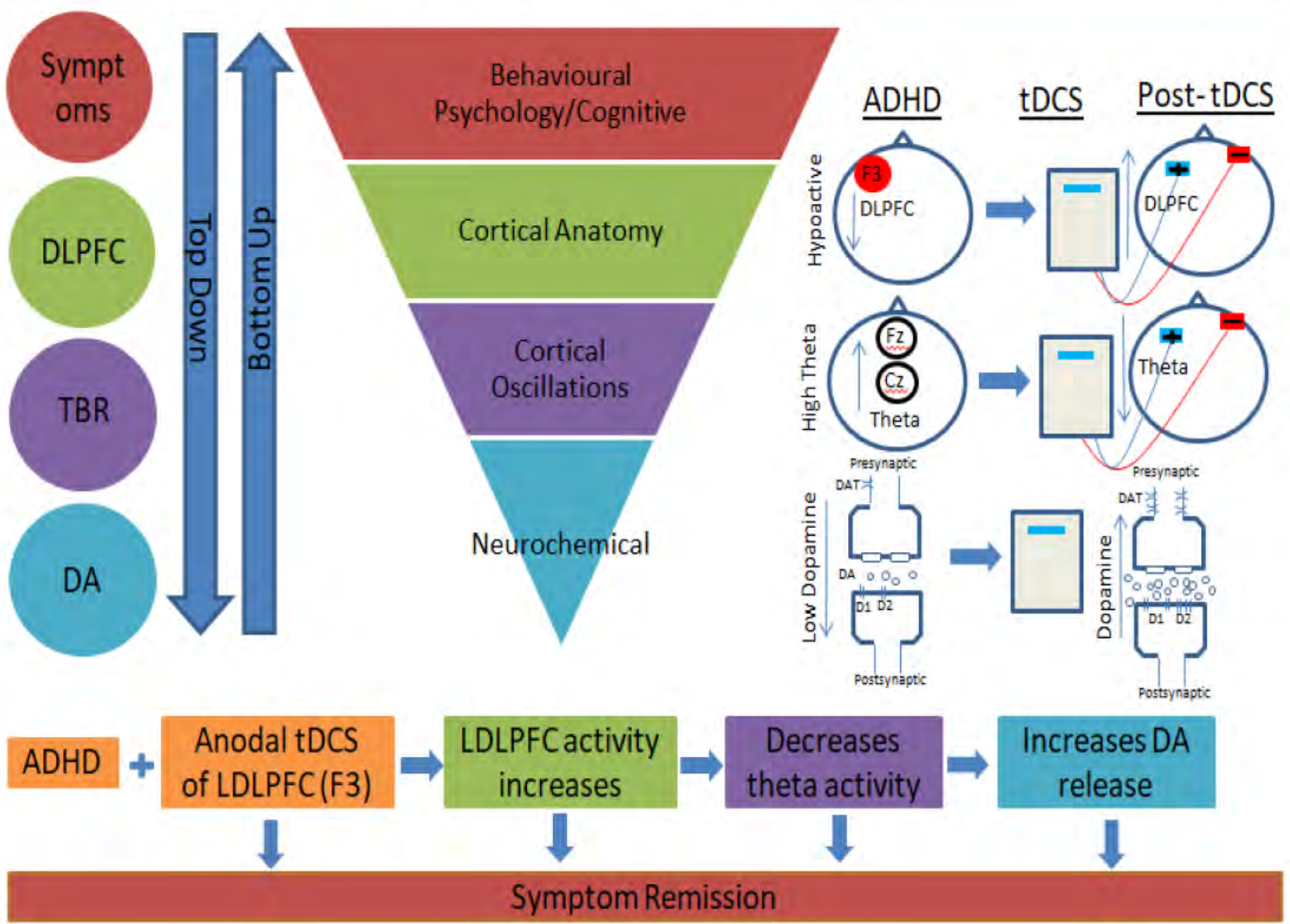

A PubMed search for "attention deficit hyperactivity disorder transcranial direct current stimulation" will yield 48 results (September 2020) with only about a dozen empirical trials - the first being from 2004. Over the next 10 years however only a handful of papers were published, each taking a preclinical cognitive neuroscience approach. Before launching into tDCS ADHD clinical trials researchers first investigated the effects of tDCS on cognitive domains related to tDCS such as: modulating inhibitory control by stimulating the superior medial frontal cortex, 
and modulating behavioural inhibition using cognitive training combined with tDCS over the right inferior frontal gyrus. The success of these studies incrementally led to the first clinical trial of tDCS for ADHD in children in 2014 which found improvements in declarative memory consolidation in children with ADHD compared to healthy controls [85]. From 2014-2020 eleven studies utilizing tDCS in ADHD were published which have been thoroughly summarized in a 2019 meta-analysis [86], and 2020 systematic review [87].

The results from the aforementioned systematic review and meta-analysis have generally sorted papers into two ADHD categories that coincide with the two psychological ADHD subtypes. The first category is tDCS effects on inhibitory control (hyperactive subtype), and the second is tDCS effects on working memory (inattentive subtype). $78 \%$ of the studies investigating inhibitory control targeted F3 dorsolateral prefrontal cortex, the remainder targeted F8 right inferior frontal gyrus. $100 \%$ of the studies aiming to improve working memory targeted F3. All but one of these experiments reported by Salehinejad et al. [86] were conducted in children, total overall $\mathrm{N}=132.54 .5 \%$ of the studies only exposed participants to a single session of tDCS (8-30 min, $1 \mathrm{~mA})$ with the max number of sessions in any study being 5 . This is important to note as a real tDCS dose for treating ADHD would likely consist of at least 20 sessions, 5 days a week, for 4 weeks.

Based on these articles $[86,87]$ it is evident that tDCS protocols for ADHD are still limited to the psychological level + network level. Although these top down approaches have been sufficient for gathering preliminary evidence about tDCS in ADHD it is possible to further optimize tDCS treatments by going a level lower to target EEG oscillations in the cortex. The TBR hypothesis is a prime example. The final study in the present thesis will explore how TBR 
may be used to inform and optimize tDCS treatments for ADHD and whether or not it is actually valid for this purpose.

\section{TBR in ADHD}

Combined with electroencephalography tDCS can anatomically and functionally target specific electrophysiological biomarkers offering an improvement to stand alone tDCS [88-90]. Given the heterogeneity of ADHD [59,91], validation of a biomarker that can provide a more homogenous phenotype would be useful for treatment and diagnostic models. To that end, the most highly cited yet controversial EEG biomarker for ADHD is the theta/beta ratio (TBR) [64]. The TBR is an electrophysiological biomarker derived from EEG by dividing theta power (microvoltage measured between $4-8 \mathrm{~Hz}$ associated with inattention drowsiness) by beta power (microvoltage measured between $12-30 \mathrm{~Hz}$ associated with awake/attentive). If validated, TBR could be a viable target for tDCS, TMS, or NFB. Individualizing psychiatric treatments based on phenotypes within a disease is consistent with the Research Domain Criteria (RDoC) framework [92] recommended by the National Institute of Mental Health for new modes of investigating mental health disorders. Basically, the RDoC approach aims to combine behavioural neuroscience with psychopathology in order to move toward a more integrative definition of diseases and their phenotypes as opposed to a purely clinical or purely systems level explanation of disease. As we saw in the previous section there are several levels for understanding tDCS effects and mechanisms and elucidating these multidimensional relationships is critical to optimizing our understanding of ADHD. However, in order to be clinically useful the proposed biomarker or phenotype needs to be rigorously tested and clearly validated. Individualizing tDCS protocols based on biomarkers and thresholds measured from scalp potentials is really an adaptation from the standardized practice used in TMS to optimize dose response curves by 
locating the M1 (motor cortex) hotspot by measuring the threshold of the motor evoked response (MEP) [93-95].

While tDCS and TBR have mostly been used experimentally in the laboratory both are garnering a great deal of clinical interest for ADHD. There are however several stages of research before a new treatment like tDCS or biomarker like TBR is adopted into routine clinical practice. Before tDCS can become a routine clinical tool in pediatric ADHD it is necessary to first establish that it is safe and tolerable, efficacious, and that it is acceptable among parents of children with ADHD. Similarly, for TBR to be considered a useful diagnostic biomarker and neuromodulatory treatment target in $\mathrm{ADHD}$ it needs to be validated.

Like any biomarker, the proposed clinical importance of TBR is to enhance diagnostics/prognostics and to act as a target in treatment models. Some authors have found that theta appears to be elevated compared to beta in ADHD patients versus healthy controls (HC) [96-98] and that a ratio of these values (TBR) could be used to help diagnose or predict ADHD $[63,66]$. An early meta-analysis from Synder [97] $(\mathrm{n}=1498)$ reported an effect size of 3.08 for $\mathrm{TBR}$ recorded at $\mathrm{Cz}$ (sensory motor area) for differentiating $\mathrm{ADHD}$ from healthy controls. However, the literature appears to have taken a step back since then. A number of more recent studies $[59,99,100]$ including two meta-analyses $[65,101]$ found no differences in TBR at $\mathrm{Cz}$ between healthy controls and children with ADHD. This is of course problematic because TBR cannot be considered a clinically useful biomarker in ADHD if it is equally prevalent and highly similar in healthy controls.

The efficacy of TBR at $\mathrm{Cz}$ has also been investigated as a biological index/target for clinical treatments such as neurofeedback (NFB) $[102,103]$, and methylphenidate [58] however has only recently been considered in tDCS. Arnold et al. [102] conducted a double-blind sham 
controlled neurofeedback trial $(n=39)$ in children with $\mathrm{ADHD}$ that aimed to reduce theta amplitude and increase beta amplitude (e.g., decrease the TBR) over 40 sessions of NFB (2-3x weekly). Although both active and sham neurofeedback produced a reduction in pre-post parental ratings of ADHD, active NFB was not superior to sham. Moreover, the same was replicated in a larger triple-blind sample $(\mathrm{n}=118)$, with extended results showing that ADHD scores decreased despite no significant increases or decreases in the TBR [103]. Therefore, according to most recent evidence, TBR does not appear to be clinically meaningful for children with ADHD.

In order to reconcile these negative results surrounding TBR some authors have suggested that perhaps TBR does not represent a diagnosis of ADHD but rather a specific EEG phenotype $[41,104]$. They further suggested that this EEG phenotype should be characterized by a threshold or cut off point (much like you would for high blood pressure or insulin levels). It has been proposed that patients who exceed this threshold would be considered good candidates for TBR targeted neuromodulation (e.g., NFB, tDCS, rTMS, iTBS). To that end, Bussalb et al. [41] aimed to validate the idea of a TBR threshold. They did this by clustering TBR values from 363 patients with ADHD into two clusters. Their analysis yielded a high and a low TBR cluster with a threshold of 3.706. Bussalb stated that the significance of these clusters is to validate a TBR threshold for patient selection in clinical trials in ADHD, but they failed to report any relationship between $\mathrm{ADHD}$ and the high and low clusters. In the present thesis we overcame this limitation by investigating the relationship between high and low TBR clusters and ADHD symptomology.

The current literature on TBR in ADHD is clearly controversial and one line of evidence posits that a threshold may be the solution [41]. This threshold has not yet been validated. 
Therefore, we contributed a replication and extension of Bussalb's analysis to rigorously test the validity of TBR. The aim of this study was to replicate the TBR clustering analysis of Bussalb et al. [41] in a normative sample, and to extend their analysis by investigating if ADHD symptomology could explain any of the variability in the clusters.

\section{In summary}

This thesis focussed on establishing the safety, tolerability, and acceptability of tDCS, and testing the validity of the TBR in pediatric $\mathrm{ADHD}$ and pediatric neuropsychiatry. 


\section{References}

1. Polanczyk, G.; De Lima, M.S.; Horta, B.L.; Biederman, J.; Rohde, L.A. The worldwide prevalence of ADHD: A systematic review and metaregression analysis. Am. J. Psychiatry 2007, 164, 942-948, doi:10.1176/ajp.2007.164.6.942.

2. Polanczyk, G. V.; Willcutt, E.G.; Salum, G.A.; Kieling, C.; Rohde, L.A. ADHD prevalence estimates across three decades: An updated systematic review and metaregression analysis. Int. J. Epidemiol. 2014, 43, 434-442, doi:10.1093/ije/dyt261.

3. Rubio, B.; Boes, A.D.; Laganiere, S.; Rotenberg, A.; Jeurissen, D.; Pascual-Leone, A. Noninvasive brain stimulation in pediatric attention-deficit hyperactivity disorder (ADHD): A review. J. Child Neurol. 2016, 31, 784-796.

4. Sikirica, V.; Flood, E.; Dietrich, C.N.; Quintero, J.; Harpin, V.; Hodgkins, P.; Skrodzki, K.; Beusterien, K.; Erder, M.H. Unmet Needs Associated with AttentionDeficit/Hyperactivity Disorder in Eight European Countries as Reported by Caregivers and Adolescents: Results from Qualitative Research. Patient 2015, 8, 269-281, doi:10.1007/s40271-014-0083-y.

5. Cosmo, C.; Baptista, A.F.; De Araújo, A.N.; Do Rosário, R.S.; Miranda, J.G.V.; Montoya, P.; De Sena, E.P. A randomized, double-blind, sham-controlled trial of transcranial direct current stimulation in attention-deficit/hyperactivity disorder. PLoS One 2015, 10, doi:10.1371/journal.pone.0135371.

6. Sotnikova, A.; Soff, C.; Becker, K.; Siniatchkin, M. Transcranial direct current stimulation (tDCS) in the treatment of adolescents with attention deficit hyperactivity disorder (ADHD). Clin. Neurophysiol. 2014, 125, S225-s226, doi:10.1007/s00702-0161646-y. 
7. Breitling, C.; Zaehle, T.; Dannhauer, M.; Bonath, B.; Tegelbeckers, J.; Flechtner, H.-H.; Krauel, K. Improving Interference Control in ADHD Patients with Transcranial Direct Current Stimulation (tDCS). Front. Cell. Neurosci. 2016, 10, doi:10.3389/fncel.2016.00072.

8. Soff, C.; Sotnikova, A.; Christiansen, H.; Becker, K.; Siniatchkin, M. Transcranial direct current stimulation improves clinical symptoms in adolescents with attention deficit hyperactivity disorder. J. Neural Transm. 2017, 124, doi:10.1007/s00702-016-1646-y.

9. Lee, J.C.; Kenney-Jung, D.L.; Blacker, C.J.; Doruk Camsari, D.; Lewis, C.P. Transcranial Direct Current Stimulation in Child and Adolescent Psychiatric Disorders. Child Adolesc. Psychiatr. Clin. N. Am. 2019, 28.

10. Rivera-Urbina, G.N.; Nitsche, M.A.; Vicario, C.M.; Molero-Chamizo, A. Applications of transcranial direct current stimulation in children and pediatrics. Rev. Neurosci. 2017, 28 , 173-184, doi:10.1515/revneuro-2016-0045.

11. Buchanan, D.M.; D'Angiulli, A.; Samson, A.; Maisonneuve, A.R.; Robaey, P. Acceptability of transcranial direct current stimulation in children and adolescents with ADHD: The point of view of parents. J. Health Psychol. 2020, 135910532093705, doi:10.1177/1359105320937059.

12. Giordano, J.; Bikson, M.; Kappenman, E.S.; Clark, V.P.; Coslett, H.B.; Hamblin, M.R.; Hamilton, R.; Jankord, R.; Kozumbo, W.J.; McKinley, R.A.; et al. Mechanisms and Effects of Transcranial Direct Current Stimulation. Dose. Response. 2017, 15, 1559325816685467, doi:10.1177/1559325816685467.

13. Buchanan, D.M.; Robaey, P.; D'angiulli, A. brain sciences Editorial What Do We Know about Transcranial Direct Current Stimulation for Major Depression? Brain Sci. 2020, 
Vol. 10, Page 480 2020, 10, 480, doi:10.3390/brainsci10080480.

14. Fregni, F.; Boggio, P.S.; Santos, M.C.; Lima, M.; Vieira, A.L.; Rigonatti, S.P.; Silva, M.T.A.; Barbosa, E.R.; Nitsche, M.A.; Pascual-Leone, A. Noninvasive cortical stimulation with transcranial direct current stimulation in Parkinson's disease. Mov. Disord. 2006, 21, 1693-1702, doi:10.1002/mds.21012.

15. Chi, R.P.; Snyder, A.W. Brain stimulation enables the solution of an inherently difficult problem. Neurosci. Lett. 2012, 515, 121-124, doi:10.1016/j.neulet.2012.03.012.

16. Jensen, M.P.; Sherlin, L.H.; Askew, R.L.; Fregni, F.; Witkop, G.; Gianas, A.; Howe, J.D.; Hakimian, S. Effects of non-pharmacological pain treatments on brain states. Clin. Neurophysiol. 2013, 124, 2016-2024, doi:10.1016/j.clinph.2013.04.009.

17. Kim, D.Y.; Lim, J.Y.; Kang, E.K.; You, D.S.; Oh, M.K.; Oh, B.M.; Paik, N.J. Effect of transcranial direct current stimulation on motor recovery in patients with subacute stroke. Am. J. Phys. Med. Rehabil. 2010, doi:10.1097/PHM.0b013e3181f70aa7.

18. Zhao, H.; Qiao, L.; Fan, D.; Zhang, S.; Turel, O.; Li, Y.; Li, J.; Xue, G.; Chen, A.; He, Q. Modulation of brain activity with noninvasive transcranial direct current stimulation (tDCS): Clinical applications and safety concerns. Front. Psychol. 2017, 8.

19. Nitsche, M.; Liebetanz, D.; Lang, N.; Antal, A.; Tergau, F.; Paulus, W.; Priori, A. Safety criteria for transcranial direct current stimulation (tDCS) in humans [1] (multiple letters). Clin. Neurophysiol. 2003, 114, 2220-2223, doi:10.1016/S1388-2457(03)00235-9.

20. Poreisz, C.; Boros, K.; Antal, A.; Paulus, W. Safety aspects of transcranial direct current stimulation concerning healthy subjects and patients. Brain Res. Bull. 2007, 72, 208-214, doi:10.1016/j.brainresbull.2007.01.004.

21. Brunoni, A.R.; Amadera, J.; Berbel, B.; Volz, M.S.; Rizzerio, B.G.; Fregni, F. A 
systematic review on reporting and assessment of adverse effects associated with transcranial direct current stimulation. Int J Psychophysiol 2011, 14, 1133-45, doi:10.1017/S1461145710001690.

22. Fertonani, A.; Ferrari, C.; Miniussi, C. What do you feel if I apply transcranial electric stimulation? Safety, sensations and secondary induced effects. Clin. Neurophysiol. 2015, 126, doi:10.1016/j.clinph.2015.03.015.

23. Bikson, M.; Grossman, P.; Thomas, C.; Zannou, A.L.; Jiang, J.; Adnan, T.; Mourdoukoutas, A.P.; Kronberg, G.; Truong, D.; Boggio, P.; et al. Safety of Transcranial Direct Current Stimulation: Evidence Based Update 2016. Brain Stimul. 2016, 9, 641661, doi:10.1016/j.brs.2016.06.004.

24. Aparício, L.V.M.; Guarienti, F.; Razza, L.B.; Carvalho, A.F.; Fregni, F.; Brunoni, A.R. A Systematic Review on the Acceptability and Tolerability of Transcranial Direct Current Stimulation Treatment in Neuropsychiatry Trials. Brain Stimul. 2016.

25. Buchanan, D.M.; Samson, A.; Maisonneuve R, A.; D’Angiulli, A.; Robaey, P. Acceptability of transcranial direct current stimulation in children and adolescents with ADHD: the point of view of parents. J. Health Psychol.

26. Buchanan, D.; D’Angiulli, A.; Samson, A.; Amare, S.; Gaumond, G.; Robaey, P. Making transcranial direct current stimulation treatment in atypical child and adolescent neurodevelopment a reality: Translating safety tolerability and acceptability evidence from the laboratory into the doctors office, the classroom, and home. Brain Stimul. 2019, 12, 474, doi:10.1016/j.brs.2018.12.545.

27. Gillick, B.T.; Feyma, T.; Menk, J.; Usset, M.; Vaith, A.; Wood, T.J.; Worthington, R.; Krach, L.E. Safety and feasibility of transcranial direct current stimulation in pediatric 
hemiparesis: randomized controlled preliminary study. Phys. Ther. 2015, 95, 337-49, doi:10.2522/ptj.20130565.

28. Zewdie, E.; Ciechanski, P.; Kuo, H.; Giuffre, A.; Kahl, C.; King, R.; Cole, L.; Grant, H.; Seeger, T.; Damji, O.; et al. Safety and tolerability of non-invasive neurostimulation in children. Brain Stimul. 2019, 12, 550, doi:10.1016/j.brs.2018.12.817.

29. Carlson, H.L.; Ciechanski, P.; Harris, A.D.; MacMaster, F.P.; Kirton, A. Changes in spectroscopic biomarkers after transcranial direct current stimulation in children with perinatal stroke. Brain Stimul. 2018, 11, 94-103, doi:10.1016/j.brs.2017.09.007.

30. Moliadze, V.; Andreas, S.; Lyzhko, E.; Schmanke, T.; Gurashvili, T.; Freitag, C.M.; Siniatchkin, M. Ten minutes of 1mA transcranial direct current stimulation was well tolerated by children and adolescents: Self-reports and resting state EEG analysis. Brain Res. Bull. 2015, 119, 25-33, doi:10.1016/j.brainresbull.2015.09.011.

31. Andrade, A.C.; Magnavita, G.M.; Allegro, J.V.B.N.; Neto, C.E.B.P.; Lucena, R. de C.S.; Fregni, F. Feasibility of Transcranial Direct Current Stimulation Use in Children Aged 5 to 12 Years. J. Child Neurol. 2013, 29, 0883073813503710 , doi:10.1177/0883073813503710.

32. Mattai, A.; Miller, R.; Weisinger, B.; Greenstein, D.; Bakalar, J.; Tossell, J.; David, C.; Wassermann, E.M.; Rapoport, J.; Gogtay, N. Tolerability of transcranial direct current stimulation in childhood-onset schizophrenia. Brain Stimul. 2011, 4, 275-280, doi:10.1016/j.brs.2011.01.001.

33. Gómez, L.; Vidal, B.; Maragoto, C.; Morales, L.M.; Berrillo, S.; Cuesta, H.V.; Baez, M.; Denis, M.; Marín, T.; Cabrera, Y.; et al. Non-invasive brain stimulation for children with autism spectrum disorders: A short-term outcome study. Behav. Sci. (Basel). 2017, 7, 
doi:10.3390/bs7030063.

34. Rich, T.L.; Nemanich, S.; Chen, M.; Friel, K.; Feyma, T.; Krach, L.; Nawshin, T.;

Meekins, G.; Gillick, B.T. Clinical Study Transcranial Direct Current Stimulation (tDCS)

Paired with Occupation-Centered Bimanual Training in Children with Unilateral Cerebral

Palsy: A Preliminary Study. 2018, doi:10.1155/2018/9610812.

35. Nemanich, S.T.; Rich, T.L.; Chen, C.Y.; Menk, J.; Rudser, K.; Chen, M.; Meekins, G.;

Gillick, B.T. Influence of combined transcranial direct current stimulation and motor training on corticospinal excitability in children with unilateral cerebral palsy. Front.

Hum. Neurosci. 2019, 13, doi:10.3389/fnhum.2019.00137.

36. Rouder, J.N.; Speckman, P.L.; Sun, D.; Morey, R.D.; Iverson, G. Bayesian t tests for accepting and rejecting the null hypothesis. Psychon. Bull. Rev. 2009, 16, 225-237.

37. Biel, A.L.; Friedrich, E.V.C. Why You Should Report Bayes Factors in Your Transcranial Brain Stimulation Studies. Front. Psychol. 2018, 9, 1125, doi:10.3389/fpsyg.2018.01125.

38. Dienes, Z.; Mclatchie, N. Four reasons to prefer Bayesian analyses over significance testing. Psychon. Bull. Rev. 2018, 25, 207-218, doi:10.3758/s13423-017-1266-z.

39. Glaser, B.; Strauss, A. Grounded Theory: The Discovery of Grounded Theory; 1967; ISBN 978-3-456-84906-5.

40. Taylor, B. Grounded theory. In Qualitative Research in the Health Sciences: Methodologies, Methods and Processes; 2013 ISBN 9780203777176.

41. Bussalb, A.; Collin, S.; Barthélemy, Q.; Ojeda, D.; Bioulac, S.; Blasco-Fontecilla, H.; Brandeis, D.; Purper Ouakil, D.; Ros, T.; Mayaud, L. Is there a cluster of high theta-beta ratio patients in attention deficit hyperactivity disorder? Clin. Neurophysiol. 2019, 130, 1387-1396, doi:10.1016/j.clinph.2019.02.021. 
42. First, M.B. DSM-5® Handbook of Differential Diagnosis; American Psychiatric Publishing, 2013; ISBN 1-58562-462-4.

43. Swanson, J.M.; Kinsbourne, M.; Nigg, J.; Lanphear, B.; Stefanatos, G.A.; Volkow, N.; Taylor, E.; Casey, B.J.; Castellanos, F.X.; Wadhwa, P.D. Etiologic subtypes of attentiondeficit/hyperactivity disorder: Brain imaging, molecular genetic and environmental factors and the dopamine hypothesis. Neuropsychol. Rev. 2007.

44. Loo, S.K.; McGough, J.J.; McCracken, J.T.; Smalley, S.L. Parsing heterogeneity in attention-deficit hyperactivity disorder using EEG-based subgroups. J. Child Psychol. Psychiatry Allied Discip. 2018, 59, doi:10.1111/jcpp.12814.

45. Fair, D.A.; Nigg, J.T.; Iyer, S.; Bathula, D.; Mills, K.L.; Dosenbach, N.U.F.; Schlaggar, B.L.; Mennes, M.; Gutman, D.; Bangaru, S.; et al. Distinct neural signatures detected for ADHD subtypes after controlling for micro-movements in resting state functional connectivity MRI data. Front. Syst. Neurosci. 2013, 1-31, doi:10.3389/fnsys.2012.00080.

46. Dickstein, S.G.; Bannon, K.; Xavier Castellanos, F.; Milham, M.P. The neural correlates of attention deficit hyperactivity disorder: an ALE meta-analysis. J. Child Psychol. Psychiatry 2006, 47, 1051-1062, doi:10.1111/j.1469-7610.2006.01671.x.

47. Seidman, L.J.; Valera, E.M.; Makris, N. Structural brain imaging of attentiondeficit/hyperactivity disorder. Biol. Psychiatry 2005.

48. Dunkley, B.T.; Urban, K.; Da Costa, L.; Wong, S.M.; Pang, E.W.; Taylor, M.J. Default Mode Network Oscillatory Coupling Is Increased Following Concussion. Front. Neurol. 2018, 9, 280, doi:10.3389/fneur.2018.00280.

49. Ellis, A.J.; Kinzel, C.; Salgari, G.C.; Loo, S.K. Frontal alpha asymmetry predicts inhibitory processing in youth with attention deficit/hyperactivity disorder. 
Neuropsychologia 2017, 102, doi:10.1016/j.neuropsychologia.2017.06.003.

50. Castellanos, F.X.; Proal, E. Large-scale brain systems in ADHD: Beyond the prefrontalstriatal model. Trends Cogn. Sci. 2012, 16, 17-26, doi:10.1016/j.tics.2011.11.007.

51. Salehinejad, M.A.; Ghayerin, E.; Nejati, V.; Yavari, F.; Nitsche, M.A. Domain-specific Involvement of the Right Posterior Parietal Cortex in Attention Network and Attentional Control of ADHD: A Randomized, Cross-over, Sham-controlled tDCS Study.

Neuroscience 2020, 444, 149-159, doi:10.1016/j.neuroscience.2020.07.037.

52. Sotnikova, A.; Soff, C.; Tagliazucchi, E.; Becker, K.; Siniatchkin, M. Transcranial Direct Current Stimulation Modulates Neuronal Networks in Attention Deficit Hyperactivity Disorder. Brain Topogr. 2017, 30, 656-672, doi:10.1007/s10548-017-0552-4.

53. Pliszka, S.R. The neuropsychopharmacology of attention-deficit/hyperactivity disorder. Biol. Psychiatry 2005.

54. Cortese, S. The neurobiology and genetics of Attention-Deficit/Hyperactivity Disorder (ADHD): What every clinician should know. Eur. J. Paediatr. Neurol. 2012, 16, 422-433.

55. Durston, S.; Fossella, J.A.; Mulder, M.J.; Casey, B.J.; Ziermans, T.B.; Vessaz, M.N.; Van Engeland, H. Dopamine transporter genotype conveys familial risk of attention-deficit/ hyperactivity disorder through striatal activation. J. Am. Acad. Child Adolesc. Psychiatry 2008, doi:10.1097/chi.0b013e31815a5f17.

56. Buyck, I.; Wiersema, J.R. Resting electroencephalogram in attention deficit hyperactivity disorder: Developmental course and diagnostic value. Psychiatry Res. 2014, 216, 391397, doi:10.1016/j.psychres.2013.12.055.

57. Kiiski, H.; Bennett, M.; Rueda-Delgado, L.M.; Farina, F.R.; Knight, R.; Boyle, R.; Roddy, D.; Grogan, K.; Bramham, J.; Kelly, C.; et al. EEG spectral power, but not theta/beta ratio, 
is a neuromarker for adult ADHD. Eur. J. Neurosci. 2020, 51, 2095-2109, doi:10.1111/ejn.14645.

58. Arns, M.; Vollebregt, M.A.; Palmer, D.; Spooner, C.; Gordon, E.; Kohn, M.; Clarke, S.; Elliott, G.R.; Buitelaar, J.K. Electroencephalographic biomarkers as predictors of methylphenidate response in attention-deficit/hyperactivity disorder. Eur.

Neuropsychopharmacol. 2018, doi:10.1016/j.euroneuro.2018.06.002.

59. Liechti, M.D.; Valko, L.; Müller, U.C.; Döhnert, M.; Drechsler, R.; Steinhausen, H.C.; Brandeis, D. Diagnostic value of resting electroencephalogram in attention-deficit/ hyperactivity disorder across the lifespan. Brain Topogr. 2013, doi:10.1007/s10548-012$0258-6$.

60. Clarke, A.R.; Barry, R.J.; McCarthy, R.; Selikowitz, M. Electroencephalogram differences in two subtypes of Attention-Deficit/Hyperactivity Disorder. Psychophysiology 2001, doi:10.1111/1469-8986.3820212.

61. Clarke, A.R.; Barry, R.J.; McCarthy, R.; Selikowitz, M. Excess beta activity in children with attention-deficit/hyperactivity disorder: An atypical electrophysiological group. Psychiatry Res. 2001, doi:10.1016/S0165-1781(01)00277-3.

62. Barry, R.J.; Clarke, A.R.; Johnstone, S.J.; McCarthy, R.; Selikowitz, M. Electroencephalogram theta/beta ratio and arousal in attention-deficit/hyperactivity disorder: evidence of independent processes. Biol. Psychiatry 2009, 66, 398-401, doi:10.1016/j.biopsych.2009.04.027.

63. Picken, C.; Clarke, A.R.; Barry, R.J.; McCarthy, R.; Selikowitz, M. The Theta/Beta Ratio as an Index of Cognitive Processing in Adults With the Combined Type of Attention Deficit Hyperactivity Disorder. Clin. EEG Neurosci. 2020, 51, 167-173, 
doi:10.1177/1550059419895142.

64. Saad, J.F.; Kohn, M.R.; Clarke, S.; Lagopoulos, J.; Hermens, D.F. Is the Theta/Beta EEG Marker for ADHD Inherently Flawed? J. Atten. Disord. 2018, 22, 815-826.

65. Arns, M.; Conners, C.K.; Kraemer, H.C. A Decade of EEG Theta/Beta Ratio Research in ADHD: A Meta-Analysis. J. Atten. Disord. 2013, doi:10.1177/1087054712460087.

66. Snyder, S.M.; Rugino, T.A.; Hornig, M.; Stein, M.A. Integration of an EEG biomarker with a clinician's ADHD evaluation. Brain Behav. 2015, 5, doi:10.1002/brb3.330.

67. Fukai, M.; Bunai, T.; Hirosawa, T.; Kikuchi, M.; Ito, S.; Minabe, Y.; Ouchi, Y. Endogenous dopamine release under transcranial direct-current stimulation governs enhanced attention: a study with positron emission tomography. Transl. Psychiatry 2019, 9, 1-10, doi:10.1038/s41398-019-0443-4.

68. Kirley, A.; Hawi, Z.; Daly, G.; McCarron, M.; Mullins, C.; Millar, N.; Waldman, I.; Fitzgerald, M.; Gill, M. Dopaminergic system genes in ADHD: Toward a biological hypothesis. Neuropsychopharmacology 2002, doi:10.1016/S0893-133X(02)00315-9.

69. Stagg, C.J.; Antal, A.; Nitsche, M.A. Physiology of Transcranial Direct Current Stimulation. J. ECT 2018, 34.

70. Bogaard, A.; Lajoie, G.; Boyd, H.; Morse, A.; Zanos, S.; Fetz, E. Cortical network mechanisms of anodal and cathodal transcranial direct current stimulation in awake primates. bioRxiv 2019, 516260, doi:10.1101/516260.

71. Liebetanz, D.; Fregni, F.; Monte-Silva, K.K.; Oliveira, M.B.; Amâncio-dos-Santos, Â.; Nitsche, M.A.; Guedes, R.C.A. After-effects of transcranial direct current stimulation (tDCS) on cortical spreading depression. Neurosci. Lett. 2006, 398, 85-90, doi:10.1016/j.neulet.2005.12.058. 
72. Stafford, J.; Brownlow, M.L.; Qualley, A.; Jankord, R. AMPA receptor translocation and phosphorylation are induced by transcranial direct current stimulation in rats. Neurobiol. Learn. Mem. 2018, doi:10.1016/j.nlm.2017.11.002.

73. Jackson, M.P.; Rahman, A.; Lafon, B.; Kronberg, G.; Ling, D.; Parra, L.C.; Bikson, M. Animal models of transcranial direct current stimulation: Methods and mechanisms. Clin. Neurophysiol. 2016, 127.

74. Demirtas-Tatlidede, A.; Vahabzadeh-Hagh, A.M.; Pascual-Leone, A. Can noninvasive brain stimulation enhance cognition in neuropsychiatric disorders? Neuropharmacology 2013,64 .

75. Zewdie, E.; Ciechanski, P.; Kuo, H.C.; Giuffre, A.; Kahl, C.; King, R.; Cole, L.; Godfrey, H.; Seeger, T.; Swansburg, R.; et al. Safety and tolerability of transcranial magnetic and direct current stimulation in children: Prospective single center evidence from 3.5 million stimulations. Brain Stimul. 2020, doi:10.1016/j.brs.2019.12.025.

76. Bloch, Y.; Harel, E. V.; Aviram, S.; Govezensky, J.; Ratzoni, G.; Levkovitz, Y. Positive effects of repetitive transcranial magnetic stimulation on attention in ADHD Subjects: A randomized controlled pilot study. World J. Biol. Psychiatry 2010, doi:10.3109/15622975.2010.484466.

77. Helfrich, C.; Pierau, S.S.; Freitag, C.M.; Roeper, J.; Ziemann, U.; Bender, S. Monitoring Cortical Excitability during Repetitive Transcranial Magnetic Stimulation in Children with ADHD: A Single-Blind, Sham-Controlled TMS-EEG Study. PLoS One 2012, doi:10.1371/journal.pone.0050073.

78. Hoogendam, J.M.; Ramakers, G.M.J.; Di Lazzaro, V. Physiology of repetitive transcranial magnetic stimulation of the human brain. Brain Stimul. 2010. 
79. Leuchter, A.F.; Cook, I.A.; Jin, Y.; Phillips, B. The relationship between brain oscillatory activity and therapeutic effectiveness of transcranial magnetic stimulation in the treatment of major depressive disorder. Front. Hum. Neurosci. 2013, doi:10.3389/fnhum.2013.00037.

80. Bai, Y.; Xia, X.; Kang, J.; Yin, X.; Yang, Y.; He, J.; Li, X. Evaluating the Effect of Repetitive Transcranial Magnetic Stimulation on Disorders of Consciousness by Using TMS-EEG. Front. Neurosci. 2016, 10, 473, doi:10.3389/fnins.2016.00473.

81. Ohnishi, T.; Hayashi, T.; Okabe, S.; Nonaka, I.; Matsuda, H.; Iida, H.; Imabayashi, E.; Watabe, H.; Miyake, Y.; Ogawa, M.; et al. Endogenous dopamine release induced by repetitive transcranial magnetic stimulation over the primary motor cortex: An [11C]raclopride positron emission tomography study in anesthetized macaque monkeys. Biol. Psychiatry 2004, doi:10.1016/j.biopsych.2003.09.016.

82. Lisanby, S.H.; Belmaker, R.H. Animal models of the mechanisms of action of repetitive transcranial magnetic stimulation (RTMS): Comparisons with electroconvulsive shock (ECS). In Proceedings of the Depression and Anxiety; 2000.

83. Terao, Y.; Ugawa, Y. Basic mechanisms of TMS. J. Clin. Neurophysiol. 2002.

84. Blumberger, D.M.; Vila-Rodriguez, F.; Thorpe, K.E.; Feffer, K.; Noda, Y.; Giacobbe, P.; Knyahnytska, Y.; Kennedy, S.H.; Lam, R.W.; Daskalakis, Z.J.; et al. Effectiveness of theta burst versus high-frequency repetitive transcranial magnetic stimulation in patients with depression (THREE-D): a randomised non-inferiority trial. Lancet 2018, 391, 16831692, doi:10.1016/S0140-6736(18)30295-2.

85. Prehn-Kristensen, A.; Munz, M.; Göder, R.; Wilhelm, I.; Korr, K.; Vahl, W.; Wiesner, C.D.; Baving, L. Transcranial oscillatory direct current stimulation during sleep improves 
declarative memory consolidation in children with attention-deficit/hyperactivity disorder to a level comparable to healthy controls. Brain Stimul. 2014, doi:10.1016/j.brs.2014.07.036.

86. Salehinejad, M.A.; Wischnewski, M.; Nejati, V.; Vicario, C.M.; Nitsche, M.A. Transcranial direct current stimulation in attention-deficit hyperactivity disorder: A metaanalysis of neuropsychological deficits. PLoS One 2019, 14, 1-26, doi:10.1371/journal.pone.0215095.

87. Salehinejad, M.A.; Nejati, V.; Mosayebi-Samani, M.; Mohammadi, A.; Wischnewski, M.; Kuo, M.F.; Avenanti, A.; Vicario, C.M.; Nitsche, M.A. Transcranial Direct Current Stimulation in ADHD: A Systematic Review of Efficacy, Safety, and Protocol-induced Electrical Field Modeling Results. Neurosci. Bull. 2020.

88. Ruffini, G.; Fox, M.D.; Ripolles, O.; Miranda, P.C.; Pascual-Leone, A. Optimization of multifocal transcranial current stimulation for weighted cortical pattern targeting from realistic modeling of electric fields. Neuroimage 2014, doi:10.1016/j.neuroimage.2013.12.002.

89. Rich, T.L.; Menk, J.S.; Rudser, K.D.; Chen, M.; Meekins, G.D.; Peña, E.; Feyma, T.; Bawroski, K.; Bush, C.; Gillick, B.T. Determining Electrode Placement for Transcranial Direct Current Stimulation: A Comparison of EEG- Versus TMS-Guided Methods. Clin. EEG Neurosci. 2017, 48, 367-375, doi:10.1177/1550059417709177.

90. Spanish Foundation for Neurometrics Development ADHD Electrophysiological Subtypes and Implications in Transcranial Direct-current Stimulation - Full Text View ClinicalTrials.gov Available online: https://clinicaltrials.gov/ct2/show/NCT01649232 (accessed on Jan 16, 2019). 
91. Loo, S.K.; McGough, J.J.; McCracken, J.T.; Smalley, S.L. Parsing heterogeneity in attention-deficit hyperactivity disorder using EEG -based subgroups. J. Child Psychol. Psychiatry 2018, 59, 223-231, doi:10.1111/jcpp.12814.

92. Sanislow, C.A.; Pine, D.S.; Quinn, K.J.; Kozak, M.J.; Garvey, M.A.; Heinssen, R.K.; Wang, P.S.E.; Cuthbert, B.N. Developing constructs for psychopathology research: Research domain criteria. J. Abnorm. Psychol. 2010, doi:10.1037/a0020909.

93. Wolf, S.L.; Butler, A.J.; Campana, G.I.; Parris, T.A.; Struys, D.M.; Weinstein, S.R.; Weiss, P. Intra-subject reliability of parameters contributing to maps generated by transcranial magnetic stimulation in able-bodied adults. Clin. Neurophysiol. 2004, doi:10.1016/j.clinph.2004.02.027.

94. Meincke, J.; Hewitt, M.; Batsikadze, G.; Liebetanz, D. Automated TMS hotspot-hunting using a closed loop threshold-based algorithm. Neuroimage 2016, doi:10.1016/j.neuroimage.2015.09.013.

95. Aonuma, S.; Gomez-Tames, J.; Laakso, I.; Hirata, A.; Takakura, T.; Tamura, M.; Muragaki, Y. A high-resolution computational localization method for transcranial magnetic stimulation mapping. Neuroimage 2018, doi:10.1016/j.neuroimage.2018.01.039.

96. Clarke, A.R.; Barry, R.J.; McCarthy, R.; Selikowitz, M. Children with attentiondeficit/hyperactivity disorder and comorbid oppositional defiant disorder: An EEG analysis. Psychiatry Res. 2002, doi:10.1016/S0165-1781(02)00137-3.

97. Snyder, S.M.; Hall, J.R. A meta-analysis of quantitative EEG power associated with attention-deficit hyperactivity disorder. J. Clin. Neurophysiol. 2006, doi:10.1097/01.wnp.0000221363.12503.78.

98. Arns, M.; Conners, C.K.; Kraemer, H.C. A Decade of EEG Theta/Beta Ratio Research in 
ADHD. J. Atten. Disord. 2013, 17, 374-383, doi:10.1177/1087054712460087.

99. Ogrim, G.; Kropotov, J.; Hestad, K. The quantitative EEG theta/beta ratio in attention deficit/hyperactivity disorder and normal controls: Sensitivity, specificity, and behavioral correlates. Psychiatry Res. 2012, 198, 482-488, doi:10.1016/j.psychres.2011.12.041.

100. Snyder, S.M.; Quintana, H.; Sexson, S.B.; Knott, P.; Haque, A.F.M.; Reynolds, D.A. Blinded, multi-center validation of EEG and rating scales in identifying ADHD within a clinical sample. Psychiatry Res. 2008, 159, 346-358, doi:10.1016/j.psychres.2007.05.006.

101. Loo, S.K.; Cho, A.; Hale, T.S.; McGough, J.; McCracken, J.; Smalley, S.L. Characterization of the Theta to Beta Ratio in ADHD: Identifying Potential Sources of Heterogeneity. J. Atten. Disord. 2013, 17, 384-392, doi:10.1177/1087054712468050.

102. Arnold, L.E.; Lofthouse, N.; Hersch, S.; Pan, X.; Hurt, E.; Bates, B.; Kassouf, K.; Moone, S.; Grantier, C. EEG Neurofeedback for ADHD: Double-Blind Sham-Controlled Randomized Pilot Feasibility Trial. J. Atten. Disord. 2013, 17, 410-419, doi:10.1177/1087054712446173.

103. Schönenberg, M.; Wiedemann, E.; Schneidt, A.; Scheeff, J.; Logemann, A.; Keune, P.M.; Hautzinger, M. Neurofeedback, sham neurofeedback, and cognitive-behavioural group therapy in adults with attention-deficit hyperactivity disorder: a triple-blind, randomised, controlled trial. The Lancet Psychiatry 2017, 4, 673-684, doi:10.1016/S22150366(17)30291-2.

104. Bussalb, A.; Congedo, M.; Barthélemy, Q.; Ojeda, D.; Acquaviva, E.; Delorme, R.; Mayaud, L. Clinical and experimental factors influencing the efficacy of neurofeedback in ADHD: A meta-analysis. Front. Psychiatry 2019, 10, doi:10.3389/fpsyt.2019.00035. 


\section{Chapter 2:}

Systematic Review on the Safety and Tolerability of Transcranial Direct

Current Stimulation in Children and Adolescents 


\section{Study 1}

\section{SYSTEMATIC REVIEW ON THE SAFETY AND TOLERABILITY OF TRANSCRANIAL DIRECT CURRENT STIMULATION IN CHILDREN AND ADOLESCENTS}

Buchanan, D.M.; Bogdanowiz, T.; Khanna, N.; Lockman-Dufour, G.; Robaey, P.; D’Angiulli, A. Systematic Review on the Safety and Tolerability of Transcranial Direct Current Stimulation in Children and Adolescents. In preparation for submission to Jama Pediatrics.

Authors: Derrick Matthew Buchanan ${ }^{\mathrm{a}, \mathrm{b}, \mathrm{c}}$, Thomas Bogdanowiz ${ }^{\mathrm{a}, \mathrm{b}}$, Neha Khanna ${ }^{\mathrm{a}, \mathrm{b}}$, Guillaume Lockman-Dufour ${ }^{\mathrm{a}, \mathrm{b}}$, Philippe Robaey ${ }^{\mathrm{a}, \mathrm{c}, \mathrm{d}}$, Amedeo D'Angiulli ${ }^{\mathrm{a}, \mathrm{b}}$

${ }^{a}$ Department of Neuroscience, Carleton University, Ottawa, ON, Canada;

${ }^{b}$ Neuroscience of Imagination Cognition Emotion Research Lab, Carleton University, Ottawa, ON, Canada;

${ }^{c}$ Neuropsychiatric Lab, Children's Hospital of Eastern Ontario, Ottawa, ON, Canada;

${ }^{d}$ Department of Psychiatry, University of Ottawa, Ottawa, ON, Canada.

Corresponding Author: Derrick Matthew Buchanan, Neuroscience of Imagination Cognition Emotion Research Lab, Loeb B150, 1125 Colonel By Dr, K1S 5B6, Ottawa, ON, Canada. Email: matthewbuchanan@cmail.carleton.ca

Declarations of interest: none. 
Abstract

Background: Transcranial direct current stimulation (tDCS) is a safe, tolerable, and acceptable technique in adults, however there is limited evidence for its safety in youths. Although limited, there are a handful of important empirical articles that have evaluated safety and tolerability outcomes in youth. However, a synthesis of pediatric studies designed to evaluate safety factors is not currently available.

Objective: To provide an update on the safety and tolerability of pediatric tDCS based on the current state of the literature.

Methods: Our search and report used PRISMA guidelines. Our method systematically examined investigations purposefully designed to evaluate the safety, tolerability, and acceptability of tDCS in healthy and atypical youths that were submitted to three databases, from the beginning of the database to November 2019. Safety considerations were evaluated by studies utilizing neuroimaging, physiological changes, performance on tasks, and by analyzing reported and objective side effects; tolerability via rate of adverse events; and acceptability via rate of dropouts.

Results: We report on 203 sham sessions, 864 active sessions up to $2 \mathrm{~mA}$, and 257 active hours of stimulation in 156 children. $4.4 \%$ of the active sessions were in neurotypical controls with the other $95.6 \%$ in clinical subjects.

Conclusion: In spite of the fact that the current evidence is sporadic and scarce, the presently reviewed literature provides support for the safety, tolerability, and acceptability, of tDCS in youth. Future pediatric tDCS research is encouraged.

Keywords: tDCS, children, safety, tolerability, brain stimulation 


\section{Introduction}

Transcranial direct current stimulation (tDCS) is a non-invasive neuromodulation technique considered to be safe, tolerable, and acceptable to use in adults [1-8] however it is minimally researched and still controversial in pediatrics [9]. tDCS utilizes a direct, low amplitude electrical current to enhance or depresses cell membrane excitability [10]. The interest around tDCS is driven by its potential to induce neuroplastic changes and potentially aid in the treatment or management of mood disorders like depression [11], memory deficits and motor control in Parkinson's [12], memory deterioration in Alzheimer's Dementia [13], impairments in attention deficit hyperactivity disorder (ADHD) [14], reduction of pain in chronic pain conditions [15], and expediting stroke recovery [16]. With the mounting experimental evidence for tDCS use in adults its attention for applications in children is growing considerably [7,17]. Rivera-Urbina et al [17] highlighted evidence for pediatric tDCS in epilepsy, autism, dislexia, $\mathrm{ADHD}$, and other psychiatric disorders like depression and schizophrenia. This is further supported by the review from Lee et al [18] which presented evidence for overall interest and efficacy of tDCS in child and adolescent psychiatric disorders. Despite the growing evidence, there is still a degree of uncertainty regarding tDCS use in children. Such reservations are primarily centered on the present lack of evidence for the short and long term safety and tolerability of tDCS in the developing brain.

\section{Current Safety Evidence}

In the last 13 years there have been a few very important safety reviews for tDCS in adults $[2,3,5,6]$ and children $[5,7,19]$. The first from Poriesz et al [2] reported frequency of side effects during and after tDCS in 102 participants across 567 sessions and demonstrated that 
tDCS is only associated with very minor side effects like scalp tingling (70.6\%), itching (30.4\%), and fatigue (35.3\%). In rare cases some minor adverse events like headache (11.8\%) or trouble sleeping emerged (0.98\%). These side effects are summarized in below in Table 1 .

Table 1. Table of common, uncommon, and rare side effects previously investigated in tDCS for adults and children.

\begin{tabular}{lll}
\hline Common & Uncommon & Rare and/or Serious \\
\hline Tingling & Headache & Visual perceptual changes \\
Itching & Nausea & Transient decrease in attention \\
Redness at site & Small blisters at the site & Transient decrease in memory \\
Discomfort & Nervousness & Difficulty concentrating \\
Mild burning sensation & Sleep disturbance & Mood changes \\
\hline
\end{tabular}

Another important review from Bikson et al [5] extensively built on these results with nearly 7000 subjects (more than 1000 of which were exposed to 5 or more sessions), including $33,000+$ total sessions: zero serious adverse events were reported. See Bikson [5] for clear criteria and a definition of serious adverse events. Bikson also reported 2800 sessions in children across nearly 500 subjects: no serious adverse events occured. Finally, Zewdie et al [19] reported no serious adverse events across 612 sessions in 92 child and adolescent subjects all collected from the same research centre. Similar to adults, itching/tingling was the most common side effect (37\%).

It is evident that the evidence in children is growing, however, the review from Bikson et al [5] also reported that less than 5\% of all tDCS research was conducted with children. This is an important discrepancy in the literature that may be due to minimal safety evidence surrounding pediatric tDCS. Some authors have indirectly reported on safety and tolerability in youth, but studies specifically designed with the intent to evaluate this are scarce. Without adequate evidence for tDCS safety in youth, researchers and clinicians will remain aptly cautious 
to use tDCS in children. The current investigation was an attempt to systematically address and respond to this discrepancy.

\section{The Current Study}

Most safety reviews to date have focussed on the broad subjective reporting of adverse events across a wide scope of the literature. Although these articles were more inclusive, it was possible to look deeper into the safety of tDCS by reviewing the outcomes of objective measures used to assess safety and tolerability: Such as pathological changes in cognition, behaviour, or neurophysiology. These factors are of particular importance for understanding the safety of tDCS in the developing brain.

Accordingly, we conducted a systematic review of tDCS safety studies in children and adolescents. In this case, safety studies were defined as studies that assessed pathological changes in physiology, and other medical evaluations like change in performance in a given physical, cognitive, or psychological domain as primary outcomes. The aim of the present review was to investigate the current consensus on the safety and tolerability of tDCS in youth based on this scope of the literature. In line with previous reviews $[2,3,5]$ tolerability was measured via rate and severity of side effects/adverse events (AEs), and acceptability via rate of dropouts. We hypothesized that there would be a positive consensus between the investigations such that tDCS is safe and tolerable in children and adolescents. 


\section{Methods}

\section{Literature Search}

This review was designed around the following primary research question: "is tDCS safe and tolerable to use in children and adolescents". To capture as many citations as possible three scholarly databases were searched: PubMed, Scopus, and Scholar Portal. The following search terms were used for all three databases: "tDCS child safety", "tDCS child tolerability", "tDCS adolescent safety", "tDCS adolescent tolerability". These search terms returned a total of 682 hits to be screened (Pubmed=155, Scopus=118, Scholar Portal=409). A graphical depiction of the study screening and selection process is illustrated in Figure 1. Finally, references in child and adolescent tDCS review articles were investigated; this did not lead to any additional articles. The literature searches and article screening process was conducted by two authors DMB and TB. References were screened from the origin of each database to November 7, 2019. This research design was derived in consultation with a professional university research librarian, Flavia Renon, and with due consideration of the preferred reporting in systematic review and meta-analyses (PRISMA) guidelines [20].

\section{Inclusion and Exclusion Criteria}

Only articles that specifically addressed safety and tolerability of pediatric tDCS via neuroimaging plus other objective outcomes were considered. The following criteria were used: (1) English manuscripts; (2) human participants below the age of 18 (we did include two studies $[21,22]$ where age ranged from 7-21 in UCP); (3) empirical research specifically reporting on child or adolescent safety, and tolerability; (4) measure and report on side effects; (5) measure and report neuroimaging outcomes. Exclusion criteria: (1) purely computational/simulated 
studies, (2) studies that did not directly measure safety and tolerability, studies using rTMS as a concurrent treatment (although studies using single-pulse TMS for neuronavigation / MEP measurements were included). One study was also included from Gomez et al [23] which used tDCS and rTMS in different groups however we only included the data from the tDCS group. The relevant tDCS data from this study were obtained through personal communication with the corresponding author of Gomez et al. 
Figure 1. Literature search PRISMA flow diagram
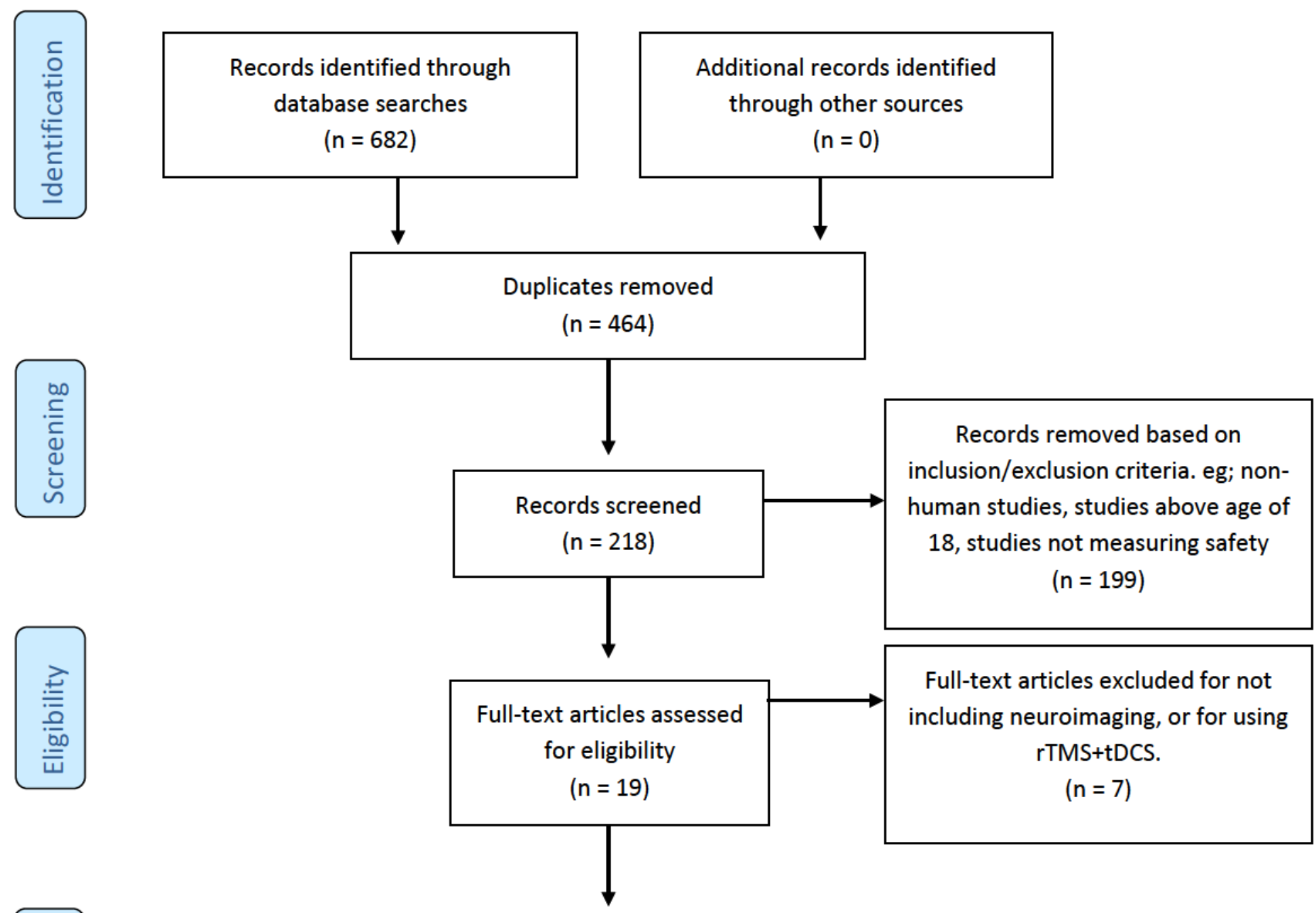

\section{Studies included in \\ qualitative synthesis \\ $(n=12)$}

Figure 1. summarizes the results yielded from our literature search. Databases were searched from inception to November 2019. Every initial paper's abstract was reviewed by two authors for eligibility for our study. Our inclusion and exclusion criteria were then applied during a second abstract and partial paper review. Finally three authors conducted a full text review and data extraction of the remaining papers. 


\section{Data Collection and Outcome Measures}

After reviewing the articles yielded from the database searches, a structured checklist of variables was developed: (1) metadata (i.e. author's names, laboratories and location, contact info, publication date and journal, one and five year impact factor); (2) participant demographics (age, number of males and females, atypical or neurotypical), (3) methods (randomized controlled trial, cognitive and psychological tests/questionnaires used, imaging techniques used, method of examining side effects, any other tasks performed); (4) tDCS protocol (amperage, sham vs. active, duration of stimulation, number of sessions, electrode location, electrode size, current density, content of saline, tDCS device used); (5) safety (outcome of neuroimaging, physiological changes, performance on tasks, rate and severity of side effects); (6) tolerability (derived outcome based on rate and description of AEs); (7) acceptability (derived outcome based on the rate and description of dropouts); (8) reported medications; (9) author's conclusions (whether pediatric tDCS is safe, tolerable, and acceptable based on their various assessments).

The main outcomes were the safety and tolerability of tDCS in youths. The secondary outcomes also included acceptability, and a verification of the influence of other variables on the primary outcomes.

\section{Risk of Assessment Bias}

In order to assess the risk of bias of among the included studies the author judged the risk of bias using Cochrane Collaboration's tool for risk of bias in randomized trials (ref). This includes selection bias, performance bias, detection bias, attrition bias, reporting bias, and any other bias. These biases were rated as low, uncertain, or high (Figure 2). 
Figure 2. Risk of bias assessment of each included study

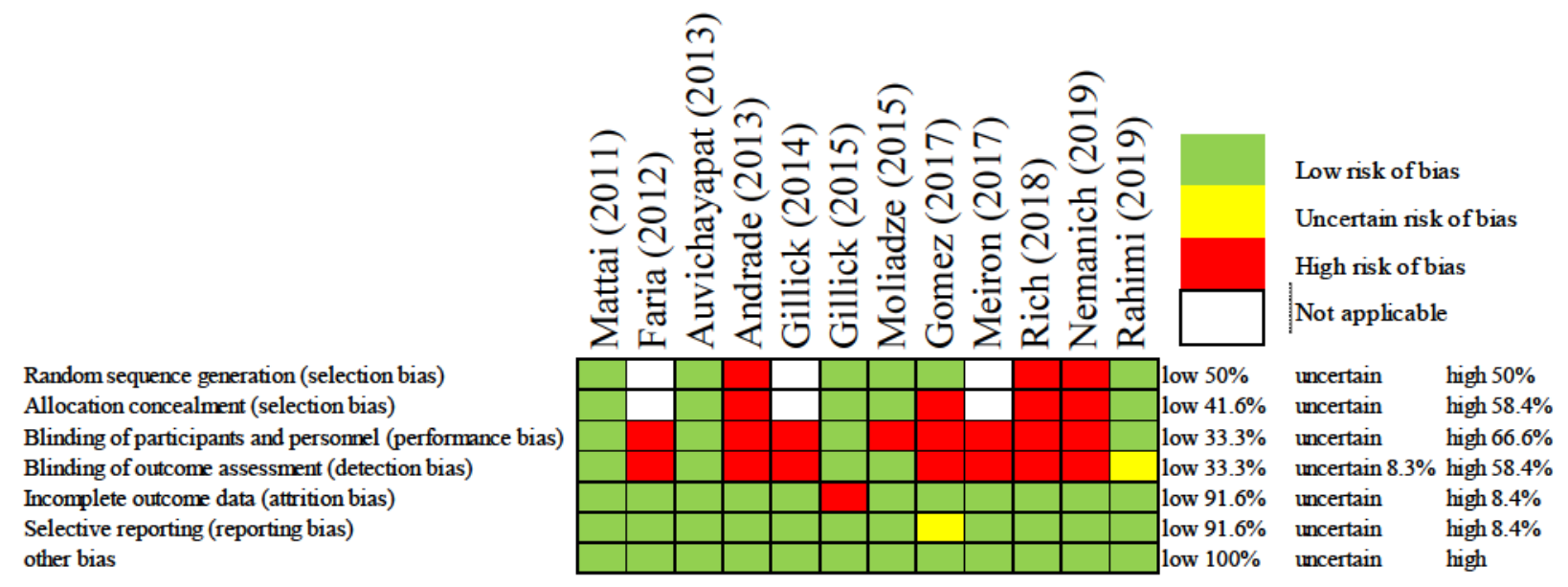

\section{Results}

\section{Literature Search}

Our initial search yielded 682 references for title and abstract review. After removing duplicates 218 abstracts were reviewed a second time to apply our inclusion and exclusion criteria. Full text review and data extraction was conducted for a total of 19 articles. Text reviews and data extraction was completed by DMB, TB, NK, and GLD. All text reviews and data extraction was verified by DMB. Discrepancies were resolved by AD. After applying our final inclusion and exclusion criteria 12 papers remained for qualitative synthesis and review (Figure 1). The full database with all 19 articles is being published separately as a data report with an open sourced database. 


\section{Quality of Studies}

The risks of bias judements associated with the included studies are displayed in Figure 2. Overall, the risk of bias was high as the majority of studies were not randomized or blinded. That being said, some of these studies had only one or two participants (refs) making randomization impractical. In this same way, other studies such as Faria's epilepsy investigation and Gillick (refs) the authors are intentionally optimizing the patient's protocol and therefore it is a necessary and apriori bias meant to improve the patient's treatment outcomes. In these cases, the judement of bias was considered "not applicable". Despite the overall high risk of bias, some studies did mitigate bias through randomization, allocation concealment, and single / double blinding. All but one of the studies had low risk for attrition bias, and reporting bias. Improvement in study design and more systematic reporting is needed in future tDCS research studies.

\section{Safety and Tolerability Literature Characteristics}

The results presented in Table 1 provide an overview of the available tDCS safety and tolerability research in children and adolescents. Of the 12 articles included, there were 7 randomized controlled trials, 156 children, and 864 active tDCS sessions $(0.5-2 \mathrm{~mA})$ totalling 257 hours of stimulation in boys and girls typically aged 6 to 17 (mean 10.75) (Table 1). It is important to remind that these numbers represent only a fraction of all the published pediatric tDCS literature $[18,19,24]$, but an important fraction which specifically investigated objective measures of safety and tolerability. Moreover, 5 of the present studies, including a total of 69 subjects, involved repeated tDCS exposure of 10 or more sessions (max 20). This is particularly important for understanding tDCS safety as the effects of tDCS are expected to aggregate over 
multiple sessions $[1,25,26]$. Additionally, only $4.4 \%$ of the subjects were neurotypical controls, the other $95.6 \%$ of subjects were from various clinical populations. Lastly, it has been demonstrated in adults that the polarizing effects of tDCS vary based on titrated amperage and the duration of the session [27-29]. It is remains to be seen if these varying polarizing effects are consistent in children, or if they have an effect on safety and tolerability. Here we report on studies stimulating for 10-20 minutes at 0-2mA with follow up measures extending up to 6 months. So far all protocols have yielded positive safety evidence. 
Table 2. tDCS exposure for age and population

\begin{tabular}{|c|c|c|c|c|c|c|c|c|}
\hline Study & $\begin{array}{l}\mathrm{N} \\
\text { (total) }\end{array}$ & $\begin{array}{l}\mathrm{N} \\
\text { (active) }\end{array}$ & $\begin{array}{l}\mathrm{N} \\
\text { (sham) }\end{array}$ & $\begin{array}{l}\mathrm{N} \\
(\mathrm{min})\end{array}$ & $\begin{array}{l}\text { Amperage } \\
(\mathrm{mA})\end{array}$ & $\begin{array}{l}\text { Age } \\
\text { (years) }\end{array}$ & Population & $\mathrm{RCT}$ \\
\hline $\begin{array}{l}\text { Mattai } \\
\text { (2011) [30] }\end{array}$ & $\begin{array}{l}12 \\
175\end{array}$ & $\begin{array}{l}5(7) \\
125\end{array}$ & $\begin{array}{l}3(2) \\
50\end{array}$ & $\begin{array}{l}20 \\
2500\end{array}$ & 2, sham & $\begin{array}{l}10-17 \\
(16.37)\end{array}$ & $\mathrm{COS}$ & Yes \\
\hline $\begin{array}{l}\text { Faria } \\
(2012)[31]\end{array}$ & $\begin{array}{l}2 \\
6\end{array}$ & $\begin{array}{l}2 \\
4\end{array}$ & $\begin{array}{l}2 \\
2\end{array}$ & $\begin{array}{l}15 \\
60\end{array}$ & $.5,1$, sham & $\begin{array}{l}7-11 \\
(9)\end{array}$ & CSWS/LKS & No \\
\hline $\begin{array}{l}\text { Auvichayapat } \\
\text { (2013) [32] }\end{array}$ & $\begin{array}{l}36 \\
36\end{array}$ & $\begin{array}{l}20(7) \\
27\end{array}$ & $\begin{array}{l}6(3) \\
9\end{array}$ & $\begin{array}{l}20 \\
540\end{array}$ & 1 & $\begin{array}{l}6-15 \\
(11.46)\end{array}$ & Epilepsy & Yes \\
\hline $\begin{array}{l}\text { Andrade } \\
(2013) \text { [33] }\end{array}$ & $\begin{array}{l}14 \\
140\end{array}$ & $\begin{array}{l}10(4) \\
140\end{array}$ & 0 & $\begin{array}{l}10 \\
1400\end{array}$ & 1,2 & $\begin{array}{l}7-12 \\
(7.57)\end{array}$ & $\begin{array}{l}\text { ELD/PDD- } \\
\text { NOS/AS/GD }\end{array}$ & No \\
\hline $\begin{array}{l}\text { Gillick } \\
\text { (2014) [34] }\end{array}$ & $\begin{array}{l}1 \\
1\end{array}$ & $\begin{array}{l}1 \\
1\end{array}$ & 0 & $\begin{array}{l}10 \\
10\end{array}$ & 0.7 & 10 & $\begin{array}{l}\text { Stroke/Hemi- } \\
\text { paretic } \mathrm{CP}\end{array}$ & Yes \\
\hline $\begin{array}{l}\text { Gillick } \\
(2015) \text { [35] }\end{array}$ & $\begin{array}{l}11 \\
11\end{array}$ & $\begin{array}{l}3(2) \\
5\end{array}$ & $\begin{array}{l}1(5) \\
6\end{array}$ & $\begin{array}{l}10 \\
50\end{array}$ & 0.7 & $\begin{array}{l}7-18 \\
(14)\end{array}$ & $\begin{array}{l}\text { Congenital } \\
\text { Hemiparesis }\end{array}$ & Yes \\
\hline $\begin{array}{l}\text { Moliadze [36] } \\
(2015)\end{array}$ & $\begin{array}{l}19 \\
57\end{array}$ & $\begin{array}{l}8(11) \\
38\end{array}$ & $\begin{array}{l}8(11) \\
19\end{array}$ & $\begin{array}{l}10 \\
380\end{array}$ & 1 & $\begin{array}{l}11-16 \\
(13.9)\end{array}$ & Neurotypical & Yes \\
\hline $\begin{array}{l}\text { Gomez } \\
\text { (2017) [23] }\end{array}$ & $\begin{array}{l}15 \\
300\end{array}$ & $\begin{array}{l}10(5) \\
300\end{array}$ & 0 & $\begin{array}{l}20 \\
6000\end{array}$ & 1 & $\begin{array}{l}5-10 \\
(7.7)\end{array}$ & ASD & Yes \\
\hline $\begin{array}{l}\text { Meiron } \\
\text { (2017) [37] }\end{array}$ & $\begin{array}{l}1 \\
10\end{array}$ & $\begin{array}{l}1 \\
10\end{array}$ & 0 & $\begin{array}{l}20 \\
200\end{array}$ & $0.1-1$ & 2.5 & $\begin{array}{l}\text { Epileptic } \\
\text { Encephalopath } \\
\text { y }\end{array}$ & No \\
\hline $\begin{array}{l}\text { Rich } \\
(2018)[21]\end{array}$ & $\begin{array}{l}8 \\
80\end{array}$ & $\begin{array}{l}3(5) \\
80\end{array}$ & 0 & $\begin{array}{l}20 \\
1600\end{array}$ & 1.5 & $\begin{array}{l}7-21 \\
(13.4)\end{array}$ & $\begin{array}{l}\text { Perinatal } \\
\text { stroke/UCP }\end{array}$ & No \\
\hline $\begin{array}{l}\text { Nemanich } \\
(2019) \text { [22] }\end{array}$ & $\begin{array}{l}20 \\
200\end{array}$ & $\begin{array}{l}5(5) \\
100\end{array}$ & $\begin{array}{l}4(6) \\
100\end{array}$ & $\begin{array}{l}20 \\
2000\end{array}$ & 0.7 , sham & $\begin{array}{l}7-21 \\
(12.75)\end{array}$ & $\begin{array}{l}\text { UCP via } \\
\text { Hemispheric } \\
\text { stroke/PVL }\end{array}$ & Yes \\
\hline $\begin{array}{l}\text { Rahimi } \\
\text { (2019) [38] }\end{array}$ & $\begin{array}{l}17 \\
51\end{array}$ & $\begin{array}{l}9(8) \\
34\end{array}$ & $\begin{array}{l}9(8) \\
17\end{array}$ & $\begin{array}{l}20 \\
680\end{array}$ & 1 , sham & $\begin{array}{l}9-12 \\
(10.35)\end{array}$ & Dyslexia & No \\
\hline Overall & $\begin{array}{l}156 \\
1067\end{array}$ & $\begin{array}{l}77(54) \\
864\end{array}$ & $\begin{array}{l}33(35) \\
203\end{array}$ & $\begin{array}{l}15420 \\
257(\mathrm{~h})\end{array}$ & 1,2 & $\begin{array}{l}6-17 \\
(10.75)\end{array}$ & & \\
\hline
\end{tabular}

Table 1 summarizes the extent of tDCS exposure for age and population. $\mathrm{N}$ (total) is the total number of individual participants on top and the total quantity of tDCS sessions below. $\mathrm{N}$ (active) and $\mathrm{N}$ (sham) represents the number of males and females (in parentheses) who received active versus sham tDCS and the number below indicates the quantity of tDCS sessions. $\mathrm{N}$ (min) indicates the duration of each single tDCS session on top, with the number below representing the number of total minutes of active tDCS exposure in each study. Amperage indicates the level of current used in the given sessions. Age is identified first as a range on top and below as an average. Population includes children and adolescents aged $6-18$ years $(\mathrm{COS}=$ Childhood Onset Schizophrenia; CSWS=Continuous Spikes and Waves during Sleep - Rare Epilepsy; LKS=Landau-Kleffner Syndrome; ELD=Expressive Language Disorder; PDDNOS=Pervasive Developmental Disorder Not Otherwise Specified; AS=Asperger Syndrome; GP=Global Dyspraxia; $\mathrm{CP}=$ Cerebral Palsy; $\mathrm{UCP}=$ Unilatereal Cerebral Palsy; $\mathrm{PVL}=$ Periventricular leukomalacia). $\mathrm{RCT}$ indicates if the experiment was a randomized controlled trial. 


\section{Safety and Electrode Location}

As part of the dosing considerations researchers and clinicians have to consider the safety and tolerability of tDCS with electrodes placed at different sites. Since each potential anatomical site may correspond to a particular physiological response, testing one site or using one montage is not entirely sufficient for drawing conclusions about each of the other potential sites. Indeed, entire literature reviews have been published on single brain regions [39]. Our review documents at least 10 unique montages corresponding to 6 brain regions: the dorsolateral prefrontal cortex (DLPFC), superior temporal gyrus (STG), Brodmann area 6 (brain region for CP5/6), Broca's area; left medial inferior frontal gyrus (LMIFG), and the primary motor cortex (M1). That being said, in the case of Auvichayapat [32] the location varied by participant by placing the cathodal electrode over the epileptogenic region and in Gillick [34,35] a bilateral M1 montage remained, however anode/cathode location was dependent on the lesioned hemisphere where the anode is always placed over the ipsilesional cortex. Rich [21] and Nemanich [22] on the other hand positioned their electrodes according the M1 TMS hotspot [40,41]. The DLPFC montage was selected to improve cognition, STG to reduce hallucinations, Brodmann area 6 to improve movement planning, LMIFG to improve language processing, and M1 to improve motor control. tDCS appears to be safe and well tolerated at each of these electrode sites. In the section Safety and Neuroimaging the notion of electrode location optimization will be further discussed.

\section{Electrode Size and Conducting Material}

Electrode size and conducting material impacts the diffusivity or locality of the stimulation via current density and therefore it was imperative to report. $100 \%$ of the present studies included precise electrode size. The majority of the reviewed studies used the common 
$5 \mathrm{X} 7\left(35 \mathrm{~cm}^{2}\right)$ or $5 \mathrm{X} 5\left(25 \mathrm{~cm}^{2}\right)$ electrodes, however $1.09 \mathrm{~cm}^{2} \mathrm{HD}$ electrode sizes were also used. Unfortunately only $58.3 \%$ articles reported the composition of their saline. Electrode preparation is important because certain solutions have been shown to be more irritable [42]. For example, water is not ideal as it contains metals that may increase the irritability of the scalp when a current is passed through them [42]. Indeed, the review from Bikson et al [5] even went so far as to exclude studies that did not use normal saline solution. Recent communications from the International Brain Stimulation Conference have actually suggested that a syringe should be used to apply saline to each electrode such that the specific amount of saline content is controlled for each subject (as opposed to soaking the entire electrode). Henceforth studies should precisely report their electrode preparation. It is therefore recommended that authors clearly report not only electrode size, but also saline and other electrode preparation materials.

\section{Safety and Medication}

Assessing medication contraindications is an important criterion for safely implementing tDCS in clinical populations. There is currently a lack of medication reporting among the present studies and tDCS literature in general. A review from McLaren (2018) clearly illustrated that medication can influence tDCS effects on tissue excitability [43] and therefore it is possible that medication may influence the safety and tolerability of tDCS. Only 2 of the present studies [30,37] actually reported specific medications including type and dose. Nemanich [22] provided medication type but not dose in the supplementary materials. In each instance the authors admitted that it is possible the medication influenced cortical excitability, but in any case no contraindications were reported. Other studies made a general "all or none" statement about medication use indicating either no medication, or medication as normal. It appears the most common approach to medication involvement in tDCS protocols is to continue medication as 
usual. So far this has yielded safe outcomes. However, it is possible that there remain unsafe medication contraindications that can only be elucidated with precise and consistent reporting of medication use among participants. In kind with the suggestions from McLaren [43] it is advisable, where applicable, that all brain stimulation studies utilize a concomitant medication log when screening participants (a template has been provided in Appendix B-2).

\section{Safety Indications for Specific tDCS Devices}

The devices used in each study were clearly reported (5 different devices used) and adverse effects do not appear to be dependent on specific tDCS devices. That being said, it is possible that certain electrode pads, or amperage limits may lead to potentially harmful ranges in current / voltage density. In general, each manufacturer has built in common safety features to help mitigate unprecedented risk that could lead to harmful stimulation. Although the materials and features used by device manufacturers vary the physics of the method remains the same. Additionally, each device has general clearance by governing bodies such as the FDA for use as an experimental device and some for clinical purposes. As long as the devices are used properly, and the electrode materials are prepared properly, by trained individuals within the evidenced amperage range of $0-2 \mathrm{~mA}$ they should each offer the same degree of tolerability. To date, there are no publications formally examining tolerability of different devices. 
Table 3. Author demographic and materials used

\begin{tabular}{|c|c|c|c|c|c|}
\hline Study & $\begin{array}{l}\text { Location of } \\
\text { research } \\
\text { and device } \\
\text { used }\end{array}$ & Medications Reported & Electrode location & $\begin{array}{l}\text { Electrode } \\
\text { size }\left(\mathrm{cm}^{2}\right)\end{array}$ & $\begin{array}{l}\text { Sponges } \\
\text { soaked } \\
\text { with }\end{array}$ \\
\hline $\begin{array}{l}\text { Mattai [30] } \\
(2011)\end{array}$ & $\begin{array}{l}\text { USA } \\
\text { Phoresor II } \\
850\end{array}$ & $\begin{array}{l}\text { Medication as usual. The } \\
\text { atypical antipsychotic } \\
\text { clozapine ranging from } \\
\text { 100-450mg. Antipsychotic } \\
\text { medication may have } \\
\text { altered cortical excitability } \\
\text { in the cohort, thereby } \\
\text { preventing the functional } \\
\text { effects of tDCS. }\end{array}$ & $\begin{array}{l}\text { Bilateral anodal } \\
\text { DLPFC to improve } \\
\text { cognitive difficulty or } \\
\text { bilateral cathode STG } \\
\text { to reduce hallucinations }\end{array}$ & 25 & $\begin{array}{l}\text { Tap water } \\
\text { or normal } \\
\text { saline }\end{array}$ \\
\hline $\begin{array}{l}\text { Faria [31] } \\
(2012)\end{array}$ & $\begin{array}{l}\text { Portugal/U } \\
\text { SA } \\
\text { Phoresor } \\
850\end{array}$ & None to be reported. & $\begin{array}{l}\text { Cathode placed at } \\
\text { CP6/CP5 }\end{array}$ & 1.09 & $\begin{array}{l}\text { Skin } \\
\text { Pure/ } \\
\text { Electrogel }\end{array}$ \\
\hline $\begin{array}{l}\text { Auvichayapat } \\
{[32]} \\
(2013)\end{array}$ & $\begin{array}{l}\text { Thailand } \\
\text { /USA } \\
\text { Soterix } \\
\text { constant } \\
\text { current }\end{array}$ & $\begin{array}{l}\text { Medication as usual. } \\
\text { Antiepileptic medication } \\
\text { regimens. }\end{array}$ & $\begin{array}{l}\text { The cathodal electrode } \\
\text { was placed over the } \\
\text { epileptogenic focus. } \\
\text { The anodal electrode } \\
\text { was placed over the } \\
\text { contralateral shoulder } \\
\text { area. }\end{array}$ & 35 & $\begin{array}{l}\text { Normal } \\
\text { saline }\end{array}$ \\
\hline $\begin{array}{l}\text { Andrade [33] } \\
\text { (2013) }\end{array}$ & $\begin{array}{l}\text { Brazil/USA } \\
\text { Striat }\end{array}$ & None to be reported. & $\begin{array}{l}\text { Anode was positioned } \\
\text { in the Broca area (mid- } \\
\text { left inferior frontal } \\
\text { gyrus) and the cathode } \\
\text { in the right supraorbital } \\
\text { area. In patient } 3 \text {, the } \\
\text { electrodes were placed } \\
\text { in the opposite } \\
\text { hemisphere (however, } \\
\text { in the same location). }\end{array}$ & 35 & $\begin{array}{l}\text { Normal } \\
\text { saline }\end{array}$ \\
\hline $\begin{array}{l}\text { Gillick [34] } \\
\text { (2014) }\end{array}$ & $\begin{array}{l}\text { USA/Cana } \\
\text { da } \\
\text { Soterix } \\
\text { LTE }\end{array}$ & $\begin{array}{l}\text { The subject was } \\
\text { developmentally normal, } \\
\text { did not have epilepsy, and } \\
\text { was not taking any } \\
\text { neuroactive medications. }\end{array}$ & $\begin{array}{l}\text { Cathode placed over } \\
\text { the contralesional } \\
\text { motor cortex and anode } \\
\text { over the ipsilesional } \\
\text { supraorbital region } \\
\text { with the intent to } \\
\text { inhibit contralesional } \\
\text { effects upon the } \\
\text { ipsilesional cortex. }\end{array}$ & 35 & $\begin{array}{l}\text { Normal } \\
\text { saline }\end{array}$ \\
\hline
\end{tabular}




\begin{tabular}{|c|c|c|c|c|c|}
\hline $\begin{array}{l}\text { Gillick [35] } \\
(2015)\end{array}$ & $\begin{array}{l}\text { USA } \\
\text { Soterix } \\
\text { LTE }\end{array}$ & $\begin{array}{l}\text { One participant was taking } \\
\text { Levetiracetam }\end{array}$ & $\begin{array}{l}\text { Primary motor cortex. } \\
\text { The cathode rubber } \\
\text { electrode was placed } \\
\text { over the M1 FDI } \\
\text { hotspot of the } \\
\text { nonlesioned } \\
\text { hemisphere, and the } \\
\text { anode rubber electrode } \\
\text { was placed over the M1 } \\
\text { FDI hotspot of the } \\
\text { lesioned hemisphere. }\end{array}$ & 35 & $\begin{array}{l}\text { Normal } \\
\text { saline / } \\
\text { disinfecte } \\
\text { d }\end{array}$ \\
\hline $\begin{array}{l}\text { Moliadze [36] } \\
(2015)\end{array}$ & $\begin{array}{l}\text { Germany/R } \\
\text { ussia } \\
\text { neuroConn } \\
\text { constant } \\
\text { current }\end{array}$ & $\begin{array}{l}\text { None to be reported. None } \\
\text { of the subjects took any } \\
\text { psychoactive drugs, } \\
\text { smoked, or drank alcohol } \\
\text { regularly. }\end{array}$ & $\begin{array}{l}\text { Anodal, cathodal, and } \\
\text { sham tDCS were } \\
\text { applied over the left } \\
\text { primary motor cortex } \\
\text { (M1, over C3). The } \\
\text { reference electrode was } \\
\text { placed over the } \\
\text { contralateral orbit. }\end{array}$ & 35 & $\begin{array}{l}\text { Normal } \\
\text { saline/ } \\
70 \% \\
\text { cellulose } \\
30 \% \\
\text { cotton } \\
\text { sponge }\end{array}$ \\
\hline $\begin{array}{l}\text { Gomez } \\
\text { [23] (2017) }\end{array}$ & $\begin{array}{l}\text { Cuba } \\
\text { Neuroconn } \\
\text { tDCS } \\
\text { stimulator }\end{array}$ & $\begin{array}{l}\text { Medication as usual. Only } \\
\text { patients with no changes in } \\
\text { their therapeutic scheme, } \\
\text { pharmacologically or non- } \\
\text { pharmacologically, were } \\
\text { accepted. Patients who } \\
\text { needed any change were } \\
\text { excluded from the trial. }\end{array}$ & $\begin{array}{l}\text { Cathode was placed } \\
\text { over area F3 (based on } \\
\text { the } 10 / 20 \text { international } \\
\text { EEG electrode system), } \\
\text { with the anode over the } \\
\text { proximal right arm. }\end{array}$ & $\begin{array}{l}\text { Not } \\
\text { specified }\end{array}$ & $\begin{array}{l}0.9 \% \\
\mathrm{NaCl} \\
\text { solution }\end{array}$ \\
\hline $\begin{array}{l}\text { Meiron [37] } \\
(2017)\end{array}$ & $\begin{array}{l}\text { Israel } \\
\text { Soterix } \\
\text { LTE }\end{array}$ & $\begin{array}{l}\text { The infant was given } \\
\text { anticonvulsants (Phenytoin } \\
\text { and Phenobarbital) with no } \\
\text { effect. Epileptic } \\
\text { medication then changed } \\
\text { to Clonazepam } \\
\text { (1.5mg/day), Vigabatrin } \\
\text { (500mg/day) and } \\
\text { Topiramate (100mg/day). }\end{array}$ & $\begin{array}{l}\text { A } 4 \times 1 \text { ring } \\
\text { configuration was } \\
\text { applied so that the } \\
\text { cathode was placed } \\
\text { over the right temporal } \\
\text { area and the four } \\
\text { anodes were situated } \\
\text { around the cathode. } \\
\text { The right hemisphere } \\
\text { received } 90 \% \text { of } \\
\text { current. }\end{array}$ & 1.09 & $\begin{array}{l}\text { Not } \\
\text { specified }\end{array}$ \\
\hline
\end{tabular}




\begin{tabular}{|c|c|c|c|c|c|}
\hline $\begin{array}{l}\text { Rich [21] } \\
(2018)\end{array}$ & $\begin{array}{l}\text { USA } \\
\text { Soterix } \\
\text { LTE }\end{array}$ & $\begin{array}{l}\text { No patients reported use of } \\
\text { medications acting on the } \\
\text { central nervous system for } \\
\text { seizure control. No patients } \\
\text { had phenol or botulinum } \\
\text { toxin injections in the six } \\
\text { months prior to the } \\
\text { experiment. }\end{array}$ & $\begin{array}{l}\text { The cathode was } \\
\text { placed on the TMS- } \\
\text { derived hotspot } \\
\text { (primary motor cortex) } \\
\text { of the non-lesioned } \\
\text { hemisphere. The } \\
\text { reference electrode was } \\
\text { placed on the } \\
\text { contralateral } \\
\text { supraorbital region. }\end{array}$ & 25 & $\begin{array}{l}\text { Not } \\
\text { specified }\end{array}$ \\
\hline $\begin{array}{l}\text { Nemanich } \\
{[22]} \\
(2019)\end{array}$ & $\begin{array}{l}\text { USA } \\
\text { Soterix } \\
\text { LTE }\end{array}$ & None to be reported. & $\begin{array}{l}\text { The cathode was } \\
\text { placed over the TMS- } \\
\text { derived motor hotspot } \\
\text { of the contralesional } \\
\text { hemisphere, and the } \\
\text { anode was placed over } \\
\text { the contralateral } \\
\text { forehead. }\end{array}$ & $\begin{array}{l}\text { Not } \\
\text { specified }\end{array}$ & $\begin{array}{l}\text { Not } \\
\text { specified }\end{array}$ \\
\hline $\begin{array}{l}\text { Rahimi } \\
{[38]} \\
(2019)\end{array}$ & $\begin{array}{l}\text { Iran } \\
\text { Electrical } \\
\text { Brain } \\
\text { Stimulator } \\
\text { Neurostim }\end{array}$ & None to be reported. & $\begin{array}{l}\text { Anode on the left } \\
\text { superior temporal gyrus } \\
\text { (STG), with the } \\
\text { cathode either placed } \\
\text { on right STG or on the } \\
\text { right shoulder }\end{array}$ & 25 & $\begin{array}{l}0.9 \% \\
\mathrm{NaCl} \\
\text { solution }\end{array}$ \\
\hline
\end{tabular}

Table 2 summarizes the authors demographic, the tDCS materials that were used, any potential medications reported, and the anode/cathode electrode placement. Location of research and device used indicate the country or countries where the research was conducted and the brand/model of tDCS device that was used. Medications reported summarizes any psychoactive substances that subjects may have been exposed to during the trial. Electrode location provides a description of the placement of the anode and cathode electrodes. Electrode size indicates the surface area of the electrode in centimeters squared. Sponges soaked with indicates the materials used to prepare the electrode sponges.

\section{Safety and Neuroimaging}

All of the studies included in this review used neuroimaging to either evaluate safety or contribute to safety by mitigating risks. Several neuroimaging modalities have been used to evaluate the safety of tDCS in children including: electroencephalography (EEG), magnetic resonance imaging (MRI), transcranial magnetic stimulation (TMS), event related potentials (ERP), electrocardiogram (EKG), electromyography (EMG), and electrooculography (EOG). 


\section{EEG}

$67 \%(n=8)$ of the studies included in our review utilized EEG to measure changes related to tDCS $[23,30-33,36-38]$. In all of these instances no pathological changes emerged. Only $58.3 \%$ of the studies reported on epileptiform activity; no such activity emerged. Indeed, in both Faria's epilepsy study [31] and Auvichayapat [32] epileptic discharges were actually significantly decreased (evaluated up to 4 weeks post stimulation). Moreover, Moliadze et al [36] investigated quantitative EEG measures including spectral power analysis. They found that tDCS decreased power in all frequency bands other than alpha, and no pathological oscillations were reported. Additionally, recent studies have investigated how EEG can be used to optimize tDCS protocols in adults through current modeling [44,45]. Given the availability and low cost of EEG it is expected that EEG combined with tDCS will become more common as an effort to optimize treatment response.

\section{MRI}

$42 \%(n=5)$ of studies used MRI to evaluate / optimize safety [21,22,30,34,35]. Mattai et al [30] indicated that they used MRI for clinical monitoring of any neurologic deterioration. They reported no changes at all. That being said, it was not clear how the authors defined neurologic deterioration. Gillick and colleagues [34,35] on the other hand demonstrated another way that MRI can be used to assess, and enhance, the safety of tDCS through risk reduction. By deriving unique MRI head models her team modeled tDCS current flow to maximize dose response curves and optimize stimulation intensity. This means that the children were only exposed to the necessary and theoretically optimal amount of current of $0.7 \mathrm{~mA}$. Given the potentially non-linear relationship of amperage intensity-dependent effects $[28,29]$ the safest 
protocol would be optimized for dose response to avoid unnecessary exposure. This is a sound way to optimize safety in any tDCS trial. Like EEG, MRI can also serve to optimize tDCS. By collecting patient specific MRI, the tDCS protocol can be optimized based on physical characteristics like head and skull morphology, and pathological characteristics like sites of brain injuries. MRI can similarly enhance TMS treatments, and be used for overall 3D neuronavigation [40].

\section{TMS}

TMS use with MRI is also common. Rich [21] and Nemanich [22] used previously obtained MRIs from patients to create 3D head models for neuronavigation allowing for precise locating of the motor cortex. In this way TMS optimized tDCS safety through risk reduction by providing precise regions of interest. Additionally, motor evoked potentials (MEPs) are a clear measurement of cortico-spinal excitability making it useful for monitoring pathological alterations after tDCS. MEPs were measured by $33 \%$ of the present studies. No pathological alterations were reported, however the results from Rich et al [21] were inconsistent and often contrary to their hypotheses. Unexpected changes in cortical excitability could be interpreted as undesirable changes in physiology and possible adverse events. However, these changes could also be interpreted as individual differences in treatment response. Nemanich [22] on the other hand reported that $100 \%$ of participant's in the active tDCS condition experienced a decrease in MEP, as hypothesized. Overall, optimizing tDCS stimulation intensity and location should make a safer and more suitable protocol by mitigating risks of individual differences. 


\section{ERP, EKG, EMG, EOG}

ERP, EKG, EMG, EOG were each only used once out of the 12 safety studies we reviewed. Mattai [30] provided an all or none type statement, noting: " neither treatment group had significant changes in respiration, blood pressure, or heart rate during, or EEG, EKG, or MRI after tDCS" (pg. 277). While encouraging, how toxicity was defined in these evaluations is not described. On the other hand, Gillick [35] clearly defined their use of EMG as an attempt to continually monitor seizures. Another advantage of this is that it allowed the various investigators to monitor seizures while remaining blinded to group assignment. No seizures or related events occurred. Lastly, Gomez [23] utilized ERP (in which EOG electrodes were simultaneously affixed) to carry out a passive oddball paradigm. Their goal was to investigate P300 amplitude and latency. ERP was only obtained from 6 subjects, each of which had normal auditory brainstem response, however one child had no ERP response. After tDCS, P300 latency was significantly shortened, and amplitude trended toward an increase. The authors interpreted these changes as positive and in line with expectations regarding abnormal functional connectivity of the frontal lobes in ASD [23].

\section{Neuroimaging Conclusion}

It is fair to say that the reporting of neuroimaging evidence in the context of tDCS safety is inconsistent at best. Particularly, some studies claim to have used imaging to monitor abnormal or pathological changes in physiology however they scarcely defined the marker or index they evaluated as an indicator of safety. On the other hand, evaluating epileptiform activity with EEG or EMG is well defined and has pathological implications that can clearly translate to tDCS safety. Future research using neuroimaging to assess the effects of tDCS should clearly 
define the structural or functional markers that they are measuring and what their expectations are. If these expectations are violated it could possibly indicate a "neuroimaging adverse event". Of course, these studies are still novel and experimental in nature and therefore defining clear clinical expectations and predictions may not be possible. In this case, the approach from Gillick [34] is highly recommended. Gillick and colleagues clearly defined and outlined the expected risks, how they were mitigated, and the criteria for stopping protocol. Stopping protocol criteria is another important safety criterion that was only reported in this one study. It is recommended that future tDCS studies consider implementing and reporting the safety criteria that would result in the researcher having to stop the session (e.g., emergence of epileptiform signatures).

A more grossly detailed account of neuroimaging methods and results are included in the appendices (Appendix A-1). Outcomes related to neuroimaging and other adverse event assessments are summarized in Table 3.

Table 4. Adverse events reports

\begin{tabular}{|c|c|c|c|c|c|c|}
\hline Study & $\begin{array}{l}\text { Neuro- } \\
\text { imaging } \\
\text { AEs }\end{array}$ & $\begin{array}{l}\text { Clinical } \\
\text { AEs }\end{array}$ & $\begin{array}{l}\text { Psychiatric } \\
\text { AEs }\end{array}$ & Cognitive AEs & $\begin{array}{l}\text { Physical } \\
\text { AEs }\end{array}$ & $\begin{array}{l}\text { Drop } \\
\text { Outs }\end{array}$ \\
\hline $\begin{array}{l}\text { Mattai } \\
\text { (2011) [30] }\end{array}$ & $0 \%$ & $0 \%$ & $0 \%$ & $\begin{array}{l}\text { Fatigue } \\
30.7 \%\end{array}$ & $\begin{array}{l}\text { Itching } \\
53.8 \% \\
\text { Tingling } \\
46.1 \%\end{array}$ & $0 \%$ \\
\hline $\begin{array}{l}\text { Faria } \\
(2012)[31]\end{array}$ & $0 \%$ & - & - & - & $\begin{array}{l}\text { Felt Something } \\
40 \%\end{array}$ & $0 \%$ \\
\hline $\begin{array}{l}\text { Auvichayapat } \\
\text { (2013) [32] }\end{array}$ & $0 \%$ & $0 \%$ & - & - & $\begin{array}{l}\text { Skin Rash } \\
2.7 \%\end{array}$ & $0 \%$ \\
\hline $\begin{array}{l}\text { Andrade } \\
(2013) \text { [33] }\end{array}$ & $0 \%$ & $0 \%$ & $\begin{array}{l}\text { Mood } \\
42.9 \% \\
\text { Irritability } \\
35.7 \%\end{array}$ & $\begin{array}{l}\text { Fatigue } \\
14.3 \% \\
\text { Trouble } \\
\text { Concentrating } \\
14.3 \%\end{array}$ & $\begin{array}{l}\text { Tingling/itching } \\
28.6 \% \\
\text { Burning } \\
14.3 \% \\
\text { Headache } \\
14.3 \% \\
\text { Scalp Pain } \\
7.1 \%\end{array}$ & $0 \%$ \\
\hline
\end{tabular}




\begin{tabular}{|c|c|c|c|c|c|c|}
\hline $\begin{array}{l}\text { Gillick } \\
\text { (2014) [34] }\end{array}$ & - & - & - & - & $0 \%$ & $0 \%$ \\
\hline $\begin{array}{l}\text { Gillick } \\
\text { (2015) [35] }\end{array}$ & $0 \%$ & $0 \%$ & $0 \%$ & $\begin{array}{l}\text { Fatigue } \\
20 \% \\
\text { Trouble } \\
\text { Concentrating } \\
20 \%\end{array}$ & $\begin{array}{l}\text { Itching and tingling } \\
0 \% \\
\text { Burning } \\
16.7 \%\end{array}$ & $8.3 \%$ \\
\hline $\begin{array}{l}\text { Moliadze [36] } \\
\text { (2015) }\end{array}$ & $0 \%$ & - & $5.3 \%$ & $\begin{array}{l}\text { Fatigue (A) } \\
15.8 \% \\
\text { Fatigue (C) } \\
31.6\end{array}$ & $\begin{array}{l}\text { Tingling (A) } \\
31.6 \% \\
\text { Burning (A) } \\
31.6 \% \\
\text { Pain (mild) (A) } \\
15.5 \% \\
\text { Tingling (C) } \\
57.9 \% \\
\text { Burning (C) } \\
26.3 \% \\
\text { Pain (mild) (C) } \\
10.5 \%\end{array}$ & - \\
\hline $\begin{array}{l}\text { Gomez } \\
(2017) \text { [23] }\end{array}$ & $0 \%$ & $0 \%$ & $0 \%$ & $0 \%$ & - & - \\
\hline $\begin{array}{l}\text { Meiron } \\
\text { (2017) [37] }\end{array}$ & $0 \%$ & $0 \%$ & - & - & $0 \%$ & $0 \%$ \\
\hline $\begin{array}{l}\text { Rich } \\
(2018) \text { [21] }\end{array}$ & $0 \%$ & $0 \%$ & $0 \%$ & $\begin{array}{l}\text { Fatigue } \\
25 \% \\
\text { Trouble } \\
\text { Concentrating } \\
12.5 \%\end{array}$ & $\begin{array}{l}\text { Felt Something } \\
37.5 \% \\
\text { Itchiness } \\
25 \% \\
\text { Tingling } \\
25 \% \\
\text { Headache } \\
25 \%\end{array}$ & $0 \%$ \\
\hline $\begin{array}{l}\text { Nemanich } \\
(2019) \text { [22] }\end{array}$ & $0 \%$ & $0 \%$ & - & - & $0 \%$ & - \\
\hline $\begin{array}{l}\text { Rahimi } \\
(2019) \text { [38] }\end{array}$ & $0 \%$ & - & - & $0 \%$ & - & $0 \%$ \\
\hline
\end{tabular}

Table 3 summarizes adverse events reported by the included studies based on several domains.

Neuroimaging=any pathological changes; Clinical=any decline in prognosis, any abnormalities from medical exams; Psychiatric=any changes in behaviour, mood, or mental status; Cognitive=any decline in cognitive function; Physical=any physical side effects, Drop outs=any participant attrition due to stimulation. The table only includes minor adverse events that did occur and were reported. If a study reported a specific value it was included in the table. If the study did not report the value a - was used. If the outcome was measured but there was nothing to report $0 \%$ was used. 


\section{Safety and Medical Examination}

In addition to neuroimaging multiple studies included baseline and follow up medical examinations such as: vital signs (pulse, blood pressure, temperature, respiration), blood work/biochemistry, clinical assessment of mental and functional status, physical exam. $41.6 \%$ of studies measured patients' vital signs in relation to tDCS [21,30,32,35,37]. In general, all vital signs remained in normal age-specific range with no significant fluctuations present. However, these were again "all or none statements"; only Meiron [37] reported the details of the vitals; but this was a case study in a 30 month old child warranting in depth details. Meiron also collected several other important data points: "The intervention was considered safe and had no negative impact on the patient's vital signs, electrolyte levels or blood biochemistry.... Neurological assessments indicated development did not change" (pg. 140). Auvichayapat [32] further confirmed no clinically relevant changes in vitals from baseline to post-test at 1 day, 2 days, and 1 month measurements in 36 subjects. Auvichayapat also employed a Quality of Life (QOL) questionnaire evaluating physical, social, emotional, and cognitive well-being. This was administered at the same aforementioned time points. There were no decreases in QOL. Andrade et al [33] provided complete medical exam before and after tDCS in 14 subjects but did not provide any statement about these outcomes; though their overall study conclusions were positive an indicative of tDCS safety.

From 80 sessions across 8 subjects with stroke / unilateral cerebral palsy (UCP), Rich et al [21] employed the Assisting Hand Assessment (AHA) and several other secondary outcome questionnaires. Only $38 \%$ of their subjects saw improvement on the AHA. Their results for efficacy were overall inconsistent; however there did not appear to be any deterioration of function. Nemanich [22] further evaluated a sample of 20 participants with UCP using the AHA 
and TMS. As noted in the TMS section, active tDCS consistently decreased MEP as predicted, however this was not correlated with increased hand function. Although there was an overall increase in AHA none were significant between the active and sham group.

\section{Safety and Psychiatric/Cognitive Outcomes}

Several assessment methods were employed across the included studies to monitor psychiatric outcomes such as pathological behaviour, mood changes, or cognitive outcomes like decreased task performance. $58.3 \%$ of studies $[21,23,30,33,35,36,38]$ reported on some psychological, neurological, or neurocognitive outcomes before or after tDCS. In the case where no side effects were present it was again common to just report an all or none statement. However, some studies did report specific cognitive side effects like fatigue (0-31.6\%), trouble concentrating (0-20\%) and psychological side effects like mood (0-42.9\%) and irritability (035.7\%). Once again, while encouraging, it would be useful for the literature if these studies provided a short description of their safety outcomes and how they defined pathological alterations in each assessment method.

A few studies employed specific questionnaires based on their samples that allowed them to evaluate systematic change in task performance or self-report. These included: California Verbal Learning Test (CVLT), Wechsler Memory Scale, clinical ratings (Symptom Assessment for Positive Symptoms [SAPS]), Brief Psychiatric Rating Scale for Children, mini mental status examination (MMSE) [30]; activities of social interaction and speech, Patient Global Impression of Improvement [33]; Autism Behavioural Checklist (ABC) and the Autism Treatment Evaluation Checklist (ATEC) [23]; Gap in Noise (GIN) test [38]. In general, none of the aforementioned measurements indicated any deterioration of verbal fluency, speech, memory, 
mental status, or behaviour. Due to the heterogeneity of these study measures they were not particularly generalizable.

\section{Tolerability and Adverse Event Reports}

Under the definition from Bikson [5] there were no serious adverse events in any of the 12 studies included in this review. $83 \%$ of the studies included some report on adverse events and tolerability. $66 \%$ of these $[21,22,30,31,33-36]$ employed relatively congruent structured questionnaires such as the one originally proposed in the Poreisz [2] safety review. These studies investigated side effects such as itching, tingling, pain, burning, rash, blisters, fatigue, insomnia, nausea, mood, difficulty concentrating, and changes in vision. These questionnaires were typically self-report and included scales for severity and duration (ie., during the session, and how long after the session). Concurrent with adult safety reviews, the most frequent side effect in children was also tingling and itching ranging from $25-57.9 \%$ and $25-53.8 \%$ respectively across the included studies. This is followed by fatigue $14.3-30.7 \%$, a burning sensation $14.3-31.6 \%$, headache 14.3-25\%, and trouble concentrating 12.5-20\%. Mattai [30] and Gillick [35] were the only studies that statistically compared side effects between active and sham groups. Neither found any significant differences between groups. This is in line with the results presented in Chapter 3. A few other studies also clearly presented similar tolerability between active and sham groups despite not comparing them statistically. Moreover, all of these side effects generally seemed to subside with cessation of stimulation. Only rarely did they persist beyond a couple of hours. Note, the studies in Table 3 only reflect the studies that reported quantitative measures; several studies simply made qualitative statements such as "participants did not experience any adverse events". 
The only minor adverse events that really stand out from our review are the changes in mood (42.9\%) and irritability (35.7\%) reported by Andrade [33]. As mood changes have not been commonly reported in tDCS it is not clear what would bring about this change in the children. Andrade addressed this discrepancy through a parent report by asking them to what degree they believed the individual side effects were related to stimulation. To that end Andrade "noted that burning sensation, scalp pain, and redness complaints were considered by the parents as definitively related to the intervention in all cases. Headache, sleepiness, and trouble concentrating were also considered to be possibly or probably related to stimulation in $50 \%$ of the cases. Other psychological symptoms, such as acute mood change and irritability, were not reported as being related to stimulation in $66.7 \%$ and $40 \%$ of the patients, respectively" ([33] pg. 1362).

\section{Acceptability and Rate of Dropouts}

$75 \%$ of the studies reported on participant attrition. In order to facilitate reviews on tDCS acceptability, and for general transparency, it is recommended that all tDCS studies make a statement about attrition. This statement should indicate "no attrition". Or, in the event of attrition, this statement should indicate the reason, and whether or not it was related to stimulation. In the present review, only 1 subject did not complete their study protocol due to discomfort. Under this definition "rate of drop outs = acceptability", tDCS appears to be very acceptable in children and adolescents. That being said, recent research, such as that presented in Chapter $4,[8,46]$ has demonstrated that defining acceptability by rate of drop outs can be limited. Specifically, parents of children who have underwent tDCS were interviewed regarding tDCS acceptability: For them, tDCS acceptability was primarily dependent on efficacy, availability, cost, and side effects compared to medication [8]. 


\section{Discussion}

In this review we advanced the literature by providing a consensus of the leading studies evaluating tDCS safety and tolerability in children. Paradoxically, the only way to address future unknown outcomes is by conducting more tDCS research in children. The current safety results, (ie., lack of any serious adverse events, and lack of neurological / psychological damage) provide support for the continuation and extrapolation of experimental and clinical tDCS use in children. This evidence appears to be quite strong and consistent for at least up to 4 weeks post tDCS in more than 10 repeated sessions up to $2 \mathrm{~mA}$. There still remain outstanding questions regarding potential long term use and long term side effects of tDCS. Thus, longitudinal studies will be of great importance to the future of the field. We encourage all future tDCS studies, regardless of primary outcome, to specifically implement components for evaluating safety and tolerability outcomes to ensure the reliability of the above results and ongoing well-being of patients. The side effect questionnaire from Poreisz [2] or Brunoni [3] should be considered a minimum standard.

Recent trends in tDCS research have focussed on at home use. Indeed, multiple studies have found good compliance and outcomes using an at home tDCS approach [47-49]. Although this raises some obvious safety concerns, Charvet et al [50] and Knotkova et al [51] have published practical guidelines for at home or remotely supervised tDCS use. These are admirable steps forward and will help researchers and clinicians safely implement tDCS at home. This is particularly important in the current climate of COVID-19. A recent guideline was published by Bikson et al [52] to outline the necessity and importance of safely implementing and continuing tDCS and TMS treatments during the pandemic. That being said, the greatest threat to tDCS safety is not likely in academia or medicine, but rather among consumers pursuing self- 
prescribed at home tDCS. If used improperly tDCS may cause burns and potentially other unforeseen adverse events.

Fortunately or unfortunately tDCS is readily available for less than $\$ 200$ via ecommerce websites like Amazon and consumer reviews seem to indicate that it is in demand. A quick YouTube search of at home tDCS will reveal videos with tens of thousands of views further attesting to consumer interest and demand. Even parents of children with ADHD have expressed their desire to pursue tDCS treatment [8]. Despite expert panels [53] these devices are available for sale without any governing authority or regulation. Aside from the warranted safety concerns, this poses a serious healthcare dilemma. At the moment, tDCS is not a readily available treatment option in most hospitals or outpatient treatment centres, but it is available for unregulated consumer purchase online. This means that individuals who desire tDCS treatment will primarily have to pursue it on their own without the guidance of a medical professional. It would seem that the adoption of tDCS into community medicine would help to ensure the safety of those pursuing tDCS as a treatment option. Therefore, there is a consistent onus on researchers to disseminate evidence into community medicine and to educate clinicians on how to properly implement tDCS into their practice. It is evident that when used properly tDCS has the capability of being a safe and effective medical intervention; there is no reason why patients should have to put themselves at risk by pursuing this treatment on their own.

Another recent trend in tDCS research is dosage: such as individualized amperage and electrode montage based on MRI, EEG, or TMS $[27,28,54-58]$. This research has revealed that tDCS, much like medication, dosage and titration can be optimized based on individual characteristics like age and weight, but also skull and brain morphology. Arguably, individualized tDCS protocols should radically improve treatment efficacy and subsequently 
safety. If individualized tDCS is considered the highest level of safety precaution, then consumer prescribed tDCS would be the least. In the present review, 5 studies used methods to individualize tDCS, such as Auvichayapat [32] placing the electrode over the epileptogenic focus, or Rich [21] using the TMS motor hot spot and MEP threshold. Although the other 7 studies did not individualize their tDCS protocols they collectively provided sound evidence for the general safety of tDCS at multiple relevant brain regions such as the DLPFC, STG, and M1.

Finally, there are some limitations of this review that should be highlighted. Firstly, although the PRISMA guidelines were duly considered in the preparation of our systematic review, no formal risk bias analysis was conducted. Similarly, the researchers involved in the literature review were not blinded. It is possible that this could have led to some selection bias however, the authors are confident our method was robust and in line with our inclusion and exclusion criteria.

\section{Conclusion}

Overall, we report on 203 sham sessions, 864 active sessions up to $2 \mathrm{~mA}$, and 257 active hours of stimulation in 156 participants. $4.4 \%$ of the active sessions were in neurotypical controls with the other $95.6 \%$ in clinical subjects. Moreover, it is evident by the participation of nearly 100 authors from 10 countries across 4 continents that the safety of pediatric tDCS is of global interest. Internationally, tDCS appears to be safe and highly tolerable with only minor levels of discomfort in a minority of children and no serious adverse events. tDCS also appears to be very acceptable with nearly all enrolled subjects completing their respective protocol. Therefore, based on the present update, tDCS appears ready for widespread research and increased clinical use in children and adolescents. 


\section{References}

1. Nitsche, M.; Liebetanz, D.; Lang, N.; Antal, A.; Tergau, F.; Paulus, W.; Priori, A. Safety criteria for transcranial direct current stimulation (tDCS) in humans [1] (multiple letters). Clin. Neurophysiol. 2003, 114, 2220-2223, doi:10.1016/S1388-2457(03)00235-9.

2. Poreisz, C.; Boros, K.; Antal, A.; Paulus, W. Safety aspects of transcranial direct current stimulation concerning healthy subjects and patients. Brain Res. Bull. 2007, 72, 208-214, doi:10.1016/j.brainresbull.2007.01.004.

3. Brunoni, A.R.; Amadera, J.; Berbel, B.; Volz, M.S.; Rizzerio, B.G.; Fregni, F. A systematic review on reporting and assessment of adverse effects associated with transcranial direct current stimulation. Int J Psychophysiol 2011, 14, 1133-45, doi:10.1017/S1461145710001690.

4. Fertonani, A.; Ferrari, C.; Miniussi, C. What do you feel if I apply transcranial electric stimulation? Safety, sensations and secondary induced effects. Clin. Neurophysiol. 2015, 126, doi:10.1016/j.clinph.2015.03.015.

5. Bikson, M.; Grossman, P.; Thomas, C.; Zannou, A.L.; Jiang, J.; Adnan, T.; Mourdoukoutas, A.P.; Kronberg, G.; Truong, D.; Boggio, P.; et al. Safety of Transcranial Direct Current Stimulation: Evidence Based Update 2016. Brain Stimul. 2016, 9, 641661, doi:10.1016/j.brs.2016.06.004.

6. Aparício, L.V.M.; Guarienti, F.; Razza, L.B.; Carvalho, A.F.; Fregni, F.; Brunoni, A.R. A Systematic Review on the Acceptability and Tolerability of Transcranial Direct Current Stimulation Treatment in Neuropsychiatry Trials. Brain Stimul. 2016.

7. Zhao, H.; Qiao, L.; Fan, D.; Zhang, S.; Turel, O.; Li, Y.; Li, J.; Xue, G.; Chen, A.; He, Q. 
Modulation of brain activity with noninvasive transcranial direct current stimulation (tDCS): Clinical applications and safety concerns. Front. Psychol. 2017, 8.

8. Buchanan, D.M.; D'Angiulli, A.; Samson, A.; Maisonneuve, A.R.; Robaey, P. Acceptability of transcranial direct current stimulation in children and adolescents with ADHD: The point of view of parents. J. Health Psychol. 2020, 135910532093705, doi:10.1177/1359105320937059.

9. Buchanan, D.; D'Angiulli, A.; Samson, A.; Amare, S.; Gaumond, G.; Robaey, P. Making transcranial direct current stimulation treatment in atypical child and adolescent neurodevelopment a reality: Translating safety tolerability and acceptability evidence from the laboratory into the doctors office, the classroom, and home. Brain Stimul. 2019, 12, 474, doi:10.1016/j.brs.2018.12.545.

10. Giordano, J.; Bikson, M.; Kappenman, E.S.; Clark, V.P.; Coslett, H.B.; Hamblin, M.R.; Hamilton, R.; Jankord, R.; Kozumbo, W.J.; McKinley, R.A.; et al. Mechanisms and Effects of Transcranial Direct Current Stimulation. Dose. Response. 2017, 15, 1559325816685467, doi:10.1177/1559325816685467.

11. Buchanan, D.M.; Robaey, P.; D'angiulli, A. brain sciences Editorial What Do We Know about Transcranial Direct Current Stimulation for Major Depression? Brain Sci. 2020, Vol. 10, Page 480 2020, 10, 480, doi:10.3390/brainsci10080480.

12. Fregni, F.; Boggio, P.S.; Santos, M.C.; Lima, M.; Vieira, A.L.; Rigonatti, S.P.; Silva, M.T.A.; Barbosa, E.R.; Nitsche, M.A.; Pascual-Leone, A. Noninvasive cortical stimulation with transcranial direct current stimulation in Parkinson's disease. Mov. Disord. 2006, 21, 1693-1702, doi:10.1002/mds.21012. 
13. Chi, R.P.; Snyder, A.W. Brain stimulation enables the solution of an inherently difficult problem. Neurosci. Lett. 2012, 515, 121-124, doi:10.1016/j.neulet.2012.03.012.

14. Soff, C.; Sotnikova, A.; Christiansen, H.; Becker, K.; Siniatchkin, M. Transcranial direct current stimulation improves clinical symptoms in adolescents with attention deficit hyperactivity disorder. J. Neural Transm. 2017, 124, doi:10.1007/s00702-016-1646-y.

15. Jensen, M.P.; Sherlin, L.H.; Askew, R.L.; Fregni, F.; Witkop, G.; Gianas, A.; Howe, J.D.; Hakimian, S. Effects of non-pharmacological pain treatments on brain states. Clin. Neurophysiol. 2013, 124, 2016-2024, doi:10.1016/j.clinph.2013.04.009.

16. Kim, D.Y.; Lim, J.Y.; Kang, E.K.; You, D.S.; Oh, M.K.; Oh, B.M.; Paik, N.J. Effect of transcranial direct current stimulation on motor recovery in patients with subacute stroke. Am. J. Phys. Med. Rehabil. 2010, doi:10.1097/PHM.0b013e3181f70aa7.

17. Rivera-Urbina, G.N.; Nitsche, M.A.; Vicario, C.M.; Molero-Chamizo, A. Applications of transcranial direct current stimulation in children and pediatrics. Rev. Neurosci. 2017, 28 , 173-184, doi:10.1515/revneuro-2016-0045.

18. Lee, J.C.; Kenney-Jung, D.L.; Blacker, C.J.; Doruk Camsari, D.; Lewis, C.P. Transcranial Direct Current Stimulation in Child and Adolescent Psychiatric Disorders. Child Adolesc. Psychiatr. Clin. N. Am. 2019, 28.

19. Zewdie, E.; Ciechanski, P.; Kuo, H.C.; Giuffre, A.; Kahl, C.; King, R.; Cole, L.; Godfrey, H.; Seeger, T.; Swansburg, R.; et al. Safety and tolerability of transcranial magnetic and direct current stimulation in children: Prospective single center evidence from 3.5 million stimulations. Brain Stimul. 2020, doi:10.1016/j.brs.2019.12.025.

20. Moher, D.; Liberati, A.; Tetzlaff, J.; Altman, D.G.; Altman, D.; Antes, G.; Atkins, D.; 
Barbour, V.; Barrowman, N.; Berlin, J.A.; et al. Preferred reporting items for systematic reviews and meta-analyses: The PRISMA statement. PLoS Med. 2009.

21. Rich, T.L.; Nemanich, S.; Chen, M.; Friel, K.; Feyma, T.; Krach, L.; Nawshin, T.; Meekins, G.; Gillick, B.T. Transcranial direct current stimulation (tDCS) paired with occupation-centered bimanual training in children with unilateral cerebral palsy: A preliminary study. Neural Plast. 2018, 2018, doi:10.1155/2018/9610812.

22. Nemanich, S.T.; Rich, T.L.; Chen, C.-Y.; Menk, J.; Rudser, K.; Chen, M.; Meekins, G.; Gillick, B.T. Influence of Combined Transcranial Direct Current Stimulation and Motor Training on Corticospinal Excitability in Children With Unilateral Cerebral Palsy. Front. Hum. Neurosci. 2019, 13, doi:10.3389/fnhum.2019.00137.

23. Gómez, L.; Vidal, B.; Maragoto, C.; Morales, L.M.; Berrillo, S.; Cuesta, H.V.; Baez, M.; Denis, M.; Marín, T.; Cabrera, Y.; et al. Non-invasive brain stimulation for children with autism spectrum disorders: A short-term outcome study. Behav. Sci. (Basel). 2017, 7, doi:10.3390/bs7030063.

24. Rivera-Urbina, G.N.; Nitsche, M.A.; Vicario, C.M.; Molero-Chamizo, A. Applications of transcranial direct current stimulation in children and pediatrics. Rev. Neurosci 2017, 28 , 173-184, doi:10.1515/revneuro-2016-0045.

25. Alwardat, M. Comments on: "Safety of repeated sessions of transcranial direct current stimulation: A systematic review." Brain Stimul. 2018, 11.

26. Ulam, F.; Shelton, C.; Richards, L.; Davis, L.; Hunter, B.; Fregni, F.; Higgins, K. Cumulative effects of transcranial direct current stimulation on EEG oscillations and attention/working memory during subacute neurorehabilitation of traumatic brain injury. 
Clin. Neurophysiol. 2015, doi:10.1016/j.clinph.2014.05.015.

27. Samani, M.M.; Agboada, D.; Jamil, A.; Kuo, M.-F.; Nitsche, M.A. Titrating the neuroplastic effects of cathodal transcranial direct current stimulation (tDCS) over the primary motor cortex. Cortex 2019, doi:10.1016/J.CORTEX.2019.04.016.

28. Jamil, A.; Batsikadze, G.; Kuo, H.I.; Labruna, L.; Hasan, A.; Paulus, W.; Nitsche, M.A. Systematic evaluation of the impact of stimulation intensity on neuroplastic after-effects induced by transcranial direct current stimulation. J. Physiol. 2017, 595, doi:10.1113/JP272738.

29. Batsikadze, G.; Moliadze, V.; Paulus, W.; Kuo, M.F.; Nitsche, M.A. Partially non-linear stimulation intensity-dependent effects of direct current stimulation on motor cortex excitability in humans. J. Physiol. 2013, doi:10.1113/jphysiol.2012.249730.

30. Mattai, A.; Miller, R.; Weisinger, B.; Greenstein, D.; Bakalar, J.; Tossell, J.; David, C.; Wassermann, E.M.; Rapoport, J.; Gogtay, N. Tolerability of transcranial direct current stimulation in childhood-onset schizophrenia. Brain Stimul. 2011, 4, 275-280, doi:10.1016/j.brs.2011.01.001.

31. Faria, P.; Fregni, F.; Sebastião, F.; Dias, A.I.; Leal, A. Feasibility of focal transcranial DC polarization with simultaneous EEG recording: Preliminary assessment in healthy subjects and human epilepsy. Epilepsy Behav. 2012, 25, 417-425, doi:10.1016/j.yebeh.2012.06.027.

32. Auvichayapat, N.; Rotenberg, A.; Gersner, R.; Ngodklang, S.; Tiamkao, S.; Tassaneeyakul, W.; Auvichayapat, P. Transcranial direct current stimulation for treatment of refractory childhood focal epilepsy. Brain Stimul. 2013, 6, 696-700, 
doi:10.1016/j.brs.2013.01.009.

33. Andrade, A.C.; Magnavita, G.M.; Allegro, J.V.B.N.; Neto, C.E.B.P.; Lucena, R. de C.S.; Fregni, F. Feasibility of Transcranial Direct Current Stimulation Use in Children Aged 5 to 12 Years. J. Child Neurol. 2013, 29, 0883073813503710 , doi:10.1177/0883073813503710.

34. Gillick, B.T.; Feyma, T.; Menk, J.; Krach, L.E. Safety of transcranial direct current stimulation in pediatric hemiparesis: determination of the method for locating the optimal stimulation site. Brain Stimul. 2014, 7, e12, doi:10.1016/j.brs.2014.01.042.

35. Gillick, B.T.; Feyma, T.; Menk, J.; Usset, M.; Vaith, A.; Wood, T.J.; Worthington, R.; Krach, L.E. Safety and feasibility of transcranial direct current stimulation in pediatric hemiparesis: randomized controlled preliminary study. Phys. Ther. 2015, 95, 337-49, doi:10.2522/ptj.20130565.

36. Moliadze, V.; Andreas, S.; Lyzhko, E.; Schmanke, T.; Gurashvili, T.; Freitag, C.M.; Siniatchkin, M. Ten minutes of 1mA transcranial direct current stimulation was well tolerated by children and adolescents: Self-reports and resting state EEG analysis. Brain Res. Bull. 2015, 119, 25-33, doi:10.1016/j.brainresbull.2015.09.011.

37. Meiron, O.; Gale, R.; Namestnic, J.; Bennet-Back, O.; David, J.; Gebodh, N.; Adair, D.; Esmaeilpour, Z.; Bikson, M. High-Definition transcranial direct current stimulation in early onset epileptic encephalopathy: a case study. Brain Inj. 2018, 32, 135-143, doi:10.1080/02699052.2017.1390254.

38. Rahimi, V.; Mohamadkhani, G.; Alaghband-Rad, J.; Kermani, F.R.; Nikfarjad, H.; Marofizade, S. Modulation of temporal resolution and speech long-latency auditory- 
evoked potentials by transcranial direct current stimulation in children and adolescents with dyslexia. Exp. Brain Res. 2019, 237, 873-882, doi:10.1007/s00221-019-05471-9.

39. Dedoncker, J.; Brunoni, A.R.; Baeken, C.; Vanderhasselt, M.-A. A Systematic Review and Meta-Analysis of the Effects of Transcranial Direct Current Stimulation (tDCS) Over the Dorsolateral Prefrontal Cortex in Healthy and Neuropsychiatric Samples: Influence of Stimulation Parameters. Brain Stimul. 2016, 9, doi:10.1016/j.brs.2016.04.006.

40. Meincke, J.; Hewitt, M.; Batsikadze, G.; Liebetanz, D. Automated TMS hotspot-hunting using a closed loop threshold-based algorithm. Neuroimage 2016, doi:10.1016/j.neuroimage.2015.09.013.

41. Aonuma, S.; Gomez-Tames, J.; Laakso, I.; Hirata, A.; Takakura, T.; Tamura, M.; Muragaki, Y. A high-resolution computational localization method for transcranial magnetic stimulation mapping. Neuroimage 2018, doi:10.1016/j.neuroimage.2018.01.039.

42. Woods, A.J.; Antal, A.; Bikson, M.; Boggio, P.S.; Brunoni, A.R.; Celnik, P.; Cohen, L.G.; Fregni, F.; Herrmann, C.S.; Kappenman, E.S.; et al. A technical guide to tDCS, and related non-invasive brain stimulation tools. Clin. Neurophysiol. 2016, 127, 1031-1048.

43. McLaren, M.E.; Nissim, N.R.; Woods, A.J. The effects of medication use in transcranial direct current stimulation: A brief review. Brain Stimul. 2018, 11.

44. Ruffini, G.; Fox, M.D.; Ripolles, O.; Miranda, P.C.; Pascual-Leone, A. Optimization of multifocal transcranial current stimulation for weighted cortical pattern targeting from realistic modeling of electric fields. Neuroimage 2014, doi:10.1016/j.neuroimage.2013.12.002.

45. Badran, B.W.; Kofmehl, E.; Borckardt, J.J.; Bikson, M.; Mullins, C.; Summers, P.; 
George, M.S. Using transcranial electrical stimulation (TES) motor threshold to potentially determine individual transcranial direct current stimulation (tDCS) dosing. Brain Stimul. 2017, 10, 509-510, doi:10.1016/j.brs.2017.01.488.

46. Sekhon, M.; Cartwright, M.; Francis, J.J. Acceptability of healthcare interventions: An overview of reviews and development of a theoretical framework. BMC Health Serv. Res. 2017, 17, doi:10.1186/s12913-017-2031-8.

47. Riggs, A.; Patel, V.; Paneri, B.; Portenoy, R.K.; Bikson, M.; Knotkova, H. At-Home Transcranial Direct Current Stimulation (tDCS) With Telehealth Support for Symptom Control in Chronically-Ill Patients With Multiple Symptoms. Front. Behav. Neurosci. 2018, doi:10.3389/fnbeh.2018.00093.

48. André, S.; Heinrich, S.; Kayser, F.; Menzler, K.; Kesselring, J.; Khader, P.H.; Lefaucheur, J.P.; Mylius, V. At-home tDCS of the left dorsolateral prefrontal cortex improves visual short-term memory in mild vascular dementia. J. Neurol. Sci. 2016, doi:10.1016/j.jns.2016.07.065.

49. Charvet, L.; Shaw, M.; Dobbs, B.; Frontario, A.; Sherman, K.; Bikson, M.; Datta, A.; Krupp, L.; Zeinapour, E.; Kasschau, M. Remotely Supervised Transcranial Direct Current Stimulation Increases the Benefit of At-Home Cognitive Training in Multiple Sclerosis. Neuromodulation 2018, doi:10.1111/ner.12583.

50. Charvet, L.E.; Kasschau, M.; Datta, A.; Knotkova, H.; Stevens, M.C.; Alonzo, A.; Loo, C.; Krull, K.R.; Bikson, M. Remotely-supervised transcranial direct current stimulation (tDCS) for clinical trials: guidelines for technology and protocols. Front. Syst. Neurosci. 2015, 9, 26, doi:10.3389/fnsys.2015.00026. 
51. Knotkova, H.; Clayton, A.; Stevens, M.; Riggs, A.; Charvet, L.E.; Bikson, M. HomeBased Patient-Delivered Remotely Supervised Transcranial Direct Current Stimulation. In Practical Guide to Transcranial Direct Current Stimulation; Springer International Publishing: Cham, 2019; pp. 379-405.

52. Bikson, M.; Hanlon, C.A.; Woods, A.J.; Gillick, B.T.; Charvet, L.; Lamm, C.; Madeo, G.; Holczer, A.; Almeida, J.; Antal, A.; et al. Guidelines for TMS/tES clinical services and research through the COVID-19 pandemic. Brain Stimul. 2020, doi:10.1016/j.brs.2020.05.010.

53. Fregni, F.; Nitsche, M.A.; Loo, C.K.; Brunoni, A.R.; Marangolo, P.; Leite, J.; Carvalho, S.; Bolognini, N.; Caumo, W.; Paik, N.J.; et al. Regulatory considerations for the clinical and research use of transcranial direct current stimulation (tDCS): Review and recommendations from an expert panel. Clin Res Regul Aff 2014, 1333, 1060-1333, doi:10.3109/10601333.2015.980944.

54. Dmochowski, J.P.; Datta, A.; Huang, Y.; Richardson, J.D.; Bikson, M.; Fridriksson, J.; Parra, L.C. Targeted transcranial direct current stimulation for rehabilitation after stroke. Neuroimage 2013, 75, 12-19, doi:10.1016/j.neuroimage.2013.02.049.

55. Kessler, S.K.; Minhas, P.; Woods, A.J.; Rosen, A.; Gorman, C.; Bikson, M. Dosage Considerations for Transcranial Direct Current Stimulation in Children: A Computational Modeling Study. PLoS One 2013, 8, 1-15, doi:10.1371/journal.pone.0076112.

56. Truong, D.Q.; Magerowski, G.; Blackburn, G.L.; Bikson, M.; Alonso-Alonso, M. Computational modeling of transcranial direct current stimulation (tDCS) in obesity: Impact of head fat and dose guidelines. NeuroImage Clin. 2013, 2, 759-766, 
doi:10.1016/j.nicl.2013.05.011.

57. Gallo, S.; B., L.; Keysers, C.; Gazzola, V. Individual respond to stimulation does matter: Combining bi-hemispheric hd tdes and sep in empathy for pain. Brain Stimul. 2015, 8, 351, doi:10.1016/j.brs.2015.01.134.

58. Rich, T.L.; Menk, J.S.; Rudser, K.D.; Chen, M.; Meekins, G.D.; Peña, E.; Feyma, T.;

Bawroski, K.; Bush, C.; Gillick, B.T. Determining Electrode Placement for Transcranial Direct Current Stimulation: A Comparison of EEG- Versus TMS-Guided Methods. Clin. EEG Neurosci. 2017, 48, 367-375, doi:10.1177/1550059417709177. 


\section{Chapter 3:}

Safety and Tolerability of Transcranial Direct Current Stimulation in Children Adolescents and Adults: A Randomized Double-Blind ShamControlled Trial 


\section{Study 2}

SAFETY AND TOLERABILITY OF TRANSCRANIAL DIRECT CURRENT STIMULATION IN CHILDREN ADOLESCENTS AND ADULTS: A RANDOMIZED DOUBLE-BLIND SHAM-CONTROLLED TRIAL

Buchanan, D.M.; Amare, S.; Gaumon, G.; D’Angiulli, A.; Robaey, P. Safety and Tolerability of Transcranial Direct Current Stimulation in Children Adolescents and Adults: A Randomized Double-Blind Sham-Controlled Trial. In preparation for submission to the Journal of Child Psychology and Psychiatry and Allied Disciplines

Authors: Derrick Matthew Buchanan ${ }^{\mathrm{a}, \mathrm{b}, \mathrm{c}}$, Sarah Amare $^{c}$, Genevieve Gaumond ${ }^{c}$, Amedeo D'Angiulli ${ }^{\mathrm{a}, \mathrm{b}}$, Philippe Robaey ${ }^{\mathrm{a}, \mathrm{c}, \mathrm{d}}$

${ }^{a}$ Department of Neuroscience, Carleton University, Ottawa, ON, Canada;

${ }^{b}$ Neuroscience of Imagination Cognition Emotion Research Lab, Carleton University, Ottawa, ON, Canada;

${ }^{c}$ Neuropsychiatric Lab, Children's Hospital of Eastern Ontario, Ottawa, ON, Canada;

${ }^{d}$ Department of Psychiatry, University of Ottawa, Ottawa, ON, Canada.

Corresponding Author: Derrick Matthew Buchanan, Neuroscience of Imagination Cognition Emotion Research Lab, Loeb B150, 1125 Colonel By Dr, K1S 5B6, Ottawa, ON, Canada. Email: matthewbuchanan@cmail.carleton.ca

Declarations of interest: none. 


\section{Abstract}

Background: Transcranial direct current stimulation (tDCS) is a non-invasive brain stimulation technique with substantial evidence for its safety and tolerability in adults. However, less than $5 \%$ of published tDCS research is in pediatrics. Safety and tolerability have to be firmly established in order to ethically use it in children.

Objective/Hypothesis: To investigate tDCS safety and tolerability between children and adults. We hypothesized that there would be no differences in safety and tolerability between children and adults.

Methods: Sixty participants aged 6-17 and 18-45 participated in a randomized double-blind sham/placebo-controlled trial. All participants were equally distributed according to gender or clinical status. They were randomly assigned to two ten minute tDCS sessions. The primary outcome of this study was the intensity and frequency of 13 side effects evaluated at 6 different time points spanning 2 weeks. Repeated measures ANOVAs and univariate ANOVAs were conducted between children/adults, males/females, clinical/healthy groups. Bayesian comparisons were computed in parallel. Effects of amperage intensity and number needed to harm were also reported.

Results: There was no significant difference between tDCS tolerability in children and adults across time: $F(5,290)=1.719, p=.130$, partial $\eta^{2}=.029$. Of these 78 univariate ANOVA comparisons, only 6 reached uncorrected significance, and 1 of these was at the one week baseline. Side effect ratings for both children and adults rarely exceeded a maximum rating of 1 'very mild'. 
Conclusions: This study provided evidence supporting the overall short term safety and tolerability of tDCS, including electrode placement and current up to $2 \mathrm{~mA}$, in children/adults, males/females, and healthy/clinical populations.

Key words: tDCS, pediatric, children, adolescents, safety, tolerability 


\section{Introduction}

Transcranial direct current stimulation (tDCS) is a non-invasive brain stimulation technique with substantial evidence for its safety and tolerability in adults up to $2 \mathrm{~mA} \mathrm{[1-6]} \mathrm{and}$ in recent reports up to $4 \mathrm{~mA}$ [7]. Perhaps most influential in this regard is the early safety review from Poriesz et al [2], and the recent review from Bikson et al [8]. Although Bikson's review provided substantial evidence for safety in adults with over 7000 subjects and $33,000+$ total sessions, it also revealed that less than $5 \%$ of all published tDCS research is in pediatrics. Within this narrow 5\% margin only a handful of studies actually measured or assessed safety and tolerability [9-18]. This disproportionately low representation of children in the literature may be partially due to the scarcity of available safety evidence. The most representative pediatric sample to date is also from Bikson et al [8] who reported on 2800 sessions in nearly 500 children: no serious adverse events were reported. The largest single site sample was also recently reported by Zewdie et al [19] which consisted of 612 tDCS sessions in 92 children: no serious adverse events were reported. As of now, it appears that children and adolescents tolerate tDCS in a similar manner as adults; with the most common side effects being itching/tingling (37\%). That being said, there has yet to be a randomized double-blind sham-controlled tDCS safety trial in children. Moreover, the safety and tolerability of tDCS in children and adults has never been compared in the same trial. Therefore, researchers and clinicians are naturally cautious about transferring typical adult tDCS protocols to children. Understanding the safety and tolerability of tDCS in children and adolescents compared to adults is imperative to its acceptability and translation into pediatric psychiatry.

To that end, the present study aimed to prospectively examine children and adults from healthy and clinical samples in a randomized double-blind sham-controlled safety protocol. 
Children and adults were assigned to multiple sessions of tDCS with randomized amperage and electrode locations based on common adult protocols [2]. We proposed that tDCS is safe (adverse events), tolerable (side effects), and acceptable (drop outs); where the operationalization of these variables can be found in previous tDCS safety literature [8]. We further hypothesized that there would be no differences between these outcomes in children vs. adults exposed to the same tDCS protocol, or between males vs. females, or clinical vs. healthy samples.

Through this design we analyzed and determined if children and adults experienced the same frequency, intensity, and type of side effects from tDCS. The present aim was not only to test that there were no differences between groups' safety and tolerability, but also to establish equality between groups by using a Bayesian approach, testing support for the null hypothesis [20-22].

\section{Methods}

\section{Participants}

Sixty participants (Table 1) were recruited from the Children's Hospital of Eastern Ontario Mental Health Patient Service Unit outpatient clinic (Mood and Anxiety, ADHD teams) as well from the community. Inclusion criteria included: ages 6-17 and 18-45 and no previous experience with tDCS (see Appendix B-2 for inclusion criteria forms). Healthy controls had no history of neuropsychiatric condition, while clinical participants had some neurodevelopmental condition (e.g., ADHD, autism). Exclusion criteria included: history of epilepsy or seizure, adverse history of migraine/headaches, unstable medical condition or any condition that may increase the risk associated with transcranial stimulation, pregnancy, cardiac condition/recent cardiac surgery, neurological conditions, brain tumour, electronic implant, metal braces, metal 
plates in the head. Before committing to the experiment, all participants were screened to ensure that they properly fit within all of these criteria. This screening was conducted verbally, in person or by phone by a member of the research team. This study was jointly approved by both the Children's Hospital of Eastern Ontario and Carleton University's Research Ethics Boards. Participants under the age 18 provided assent in addition to the consent of the parent or guardian. A parent was present during the entirety of their child's research visit. Two assent forms were available for children ages 6-12 and 12-17 for grade specific readability.

\section{Procedure}

We used a randomized double-blind sham/placebo-controlled design. All participants were randomized to two successive tDCS sessions using the neuroConn DC in either the Neuropsychiatry Lab at CHEO or the Neuroscience of Imagination Cognition Emotion Research Lab at Carleton University. The device we used has a built in safety feature which prevents it from running if the impedance is too high, and therefore electrode impedance was always kept below 2-5Kohms. Participants were seated upright in an office chair with the stimulation device placed on a desk behind them and out of site. In each session, one of six commonly used electrode montages were randomized in order to maximize transferability and generalizability. These included: 1) anode over the motor cortex (C1) and cathode over the right frontopolar cortex above the eyebrow, 2) anode over the primary somatosensory cortex (P1) and cathode over the right frontopolar cortex above the eyebrow, 3) anode over the dorsolateral prefrontal cortex (DLPFC, F3) and cathode over the right frontopolar cortex above the eyebrow, 4) anode over the primary visual cortex $(\mathrm{Oz})$ and cathode over $(\mathrm{Cz})$ central midline, 5) anode over the temporal cortex (T3) and cathode over (T4) right temporal, 6) anode over the parietal cortex (P6- 
P8) and cathode over $(\mathrm{Cz})$ central midline. Electrode locations given in brackets correspond to the standard 10-20 system [23]. The amplitude was randomly selected for each session $(0,0.5$, 1.0 and $2.0 \mathrm{~mA}$ ). Both the researcher conducting the outcomes measures and the participant were blinded to the amperage. The currents were ramped up or down over the first and last $30 \mathrm{~s}$ of stimulation. Each rubber electrode was covered in a $5 \times 7 \mathrm{~cm}$ sponge soaked in saline solution containing $0.9 \% \mathrm{NaCl}$. One or two large adjustable rubber bands were placed around the participant's head to hold the electrodes in place. Between the two sessions the participants were given a one hour break during which they were allowed to sit quietly, watch videos, or play with toys or a videogame in the laboratory or an adjacent room.

The distribution of the participants between the different amperage conditions and groups is presented in Table 1. Due to the randomization, there were no significant difference in the proportion of the different groups (pediatric vs. adult, females vs. males, controls vs. clinical) assigned to different amperages (all chi-squares ns), and no significant difference in average amplitude between groups (all t-tests ns) for each block.

Table 1. Distribution of participants across different conditions of amperages as a function of age, sex and clinical status. The average amplitude is presented in the last column.

\begin{tabular}{|l|l|l|l|l|l|l|l|}
\hline Age & First block & Sham & $.5 \mathrm{~mA}$ & $1 \mathrm{~mA}$ & $2 \mathrm{~mA}$ & Total & Average \\
\cline { 2 - 8 } & Pediatric & 4 & 8 & 13 & 5 & 30 & 0.90 \\
\cline { 2 - 8 } & Adult & 6 & 7 & 10 & 7 & 30 & 0.92 \\
\cline { 2 - 8 } & Total & 10 & 15 & 23 & 12 & 60 & 0.91 \\
\cline { 2 - 8 } & Second block & Sham & $.5 \mathrm{~mA}$ & $1 \mathrm{~mA}$ & $2 \mathrm{~mA}$ & Total & Average \\
\cline { 2 - 8 } & Pediatric & 7 & 2 & 7 & 14 & 30 & 1.20 \\
\cline { 2 - 8 } & Adult & 5 & 3 & 9 & 13 & 30 & 1.22 \\
\hline
\end{tabular}




\begin{tabular}{|c|c|c|c|c|c|c|c|}
\hline & Total & 12 & 5 & 16 & 27 & 60 & 1.21 \\
\hline \multirow[t]{8}{*}{ Sex } & First block & Sham & $.5 \mathrm{~mA}$ & $1 \mathrm{~mA}$ & $2 \mathrm{~mA}$ & Total & Average \\
\hline & Female & 5 & 10 & 11 & 4 & 30 & 0.80 \\
\hline & Male & 5 & 5 & 12 & 8 & 30 & 1.02 \\
\hline & Total & 10 & 15 & 23 & 12 & 60 & 0.91 \\
\hline & Second block & Sham & $.5 \mathrm{~mA}$ & $1 \mathrm{~mA}$ & $2 \mathrm{~mA}$ & Total & Average \\
\hline & Female & 9 & 0 & 9 & 12 & 30 & 1.10 \\
\hline & Male & 3 & 5 & 7 & 15 & 30 & 1.32 \\
\hline & Total & 12 & 5 & 16 & 27 & 60 & 1.21 \\
\hline \multirow{8}{*}{$\begin{array}{l}\text { Clinical } \\
\text { Status }\end{array}$} & First block & Sham & $.5 \mathrm{~mA}$ & $1 \mathrm{~mA}$ & $2 \mathrm{~mA}$ & Total & Average \\
\hline & Controls & 7 & 8 & 13 & 8 & 36 & 0.92 \\
\hline & Clinical & 3 & 7 & 10 & 4 & 24 & 0.90 \\
\hline & Total & 10 & 15 & 23 & 12 & 60 & 0.91 \\
\hline & Second block & Sham & $.5 \mathrm{~mA}$ & $1 \mathrm{~mA}$ & $2 \mathrm{~mA}$ & Total & Average \\
\hline & Controls & 5 & 1 & 11 & 19 & 36 & 1.38 \\
\hline & Clinical & 7 & 4 & 5 & 8 & 24 & 0.96 \\
\hline & Total & 12 & 5 & 16 & 27 & 60 & 1.21 \\
\hline
\end{tabular}

The distribution of the participants between the different electrode montages and groups is presented in Table 2. Due to the randomization, there was no significant difference in the proportion of the different groups (pediatric vs. adult, females vs. males, controls vs. clinical) assigned to different montages (all chi-squares ns) for each block. 
Table 2. Distribution of participants across different electrode locations as a function of age, sex and clinical status

\begin{tabular}{|c|c|c|c|c|c|c|c|c|}
\hline \multirow[t]{8}{*}{ Age } & First block & Motor & Somatosensory & DLPFC & Visual & Temporal & Parietal & Total \\
\hline & Pediatric & 7 & 3 & 13 & 3 & 3 & 1 & 30 \\
\hline & Adult & 8 & 6 & 4 & 4 & 3 & 5 & 30 \\
\hline & Total & 15 & 9 & 17 & 7 & 6 & 6 & 60 \\
\hline & $\begin{array}{l}\text { Second } \\
\text { block }\end{array}$ & Motor & Somatosensory & DLPFC & Visual & Temporal & Parietal & Total \\
\hline & Pediatric & 1 & 7 & 3 & 2 & 13 & 4 & 30 \\
\hline & Adult & 3 & 3 & 6 & 7 & 6 & 5 & 30 \\
\hline & Total & 4 & 10 & 9 & 9 & 19 & 9 & 60 \\
\hline \multirow[t]{8}{*}{ Sex } & First block & Motor & Somatosensory & DLPFC & Visual & Temporal & Parietal & Total \\
\hline & Female & 10 & 7 & 6 & 3 & 2 & 2 & 30 \\
\hline & Male & 5 & 2 & 11 & 4 & 4 & 4 & 30 \\
\hline & Total & 15 & 9 & 17 & 7 & 6 & 6 & 60 \\
\hline & $\begin{array}{l}\text { Second } \\
\text { block }\end{array}$ & Motor & Somatosensory & DLPFC & Visual & Temporal & Parietal & Total \\
\hline & Female & 3 & 6 & 4 & 4 & 8 & 5 & 30 \\
\hline & Male & 1 & 4 & 5 & 5 & 11 & 4 & 30 \\
\hline & Total & 4 & 10 & 9 & 9 & 19 & 9 & 60 \\
\hline \multirow{4}{*}{$\begin{array}{l}\text { Clinical } \\
\text { Status }\end{array}$} & First block & Motor & Somatosensory & DLPFC & Visual & Temporal & Parietal & Total \\
\hline & Healthy & 8 & 5 & 11 & 5 & 4 & 3 & 36 \\
\hline & Clinical & 7 & 4 & 6 & 2 & 2 & 3 & 24 \\
\hline & Total & 15 & 9 & 17 & 7 & 6 & 6 & 60 \\
\hline
\end{tabular}




\begin{tabular}{|l|l|l|l|l|l|l|l|l|}
\hline $\begin{array}{l}\text { Second } \\
\text { block }\end{array}$ & Motor & Somatosensory & DLPFC & Visual & Temporal & Parietal & Total \\
\cline { 2 - 8 } & Healthy & 3 & 6 & 5 & 7 & 10 & 5 & 36 \\
\cline { 2 - 8 } & Clinical & 1 & 4 & 4 & 2 & 9 & 4 & 24 \\
\cline { 2 - 8 } & Total & 4 & 10 & 9 & 9 & 19 & 9 & 60 \\
\hline
\end{tabular}

In order to test the effect of amperage between groups, we pooled together the sham condition and the $0.5 \mathrm{~mA}$ conditions (Low Amperage), and the 1 and $2 \mathrm{~mA}$ conditions (High Amperage). The distribution of the participants between the Low and High Amperage group and the other groups (Age, Clinical, Sex) is presented in Table 3. Due to randomization, there was no significant difference in the proportion of the different groups (pediatric vs. adult, females vs. males, controls vs. clinical) assigned to the Low and High amperage groups (all chi-squares ns) for each block. For the Low Amperage group the mean amplitude was $0.30 \mathrm{~mA}$ (standard deviation $=0.25 \mathrm{~mA})$, and for the High Amperage $1.34 \mathrm{~mA}($ standard deviation $=0.25 \mathrm{~mA})$.

Table 3. Distribution of participants across different amperages as a function of age, sex and clinical status

\begin{tabular}{|l|l|l|l|l|}
\hline & Amperage group & Low & High & Total \\
\hline \multirow{2}{*}{ Age } & Pediatric & 12 & 18 & 30 \\
\cline { 2 - 5 } & Adult & 13 & 17 & 30 \\
\hline Sex & Female & 15 & 15 & 30 \\
\cline { 2 - 6 } & Male & 10 & 20 & 30 \\
\hline Clinical Status & Controls & 15 & 21 & 36 \\
\cline { 2 - 6 } & Clinical & 10 & 14 & 24 \\
\hline & Group total & 25 & 35 & 60 \\
\hline
\end{tabular}




\section{Outcome Measures}

The primary outcome of this study was the intensity and frequency of side effects up to one week following the tDCS intervention. Side effects were evaluated at six different time points: one week before the tDCS sessions (T1), immediately before the first tDCS session (T2), immediately after their first session (T3), after a one hour break just before the second session (T4), T5 immediately after the second session, one week following the tDCS sessions (T6). Side effects were quantitatively evaluated using a questionnaire comprising 13 side effects, ranging from common to rare, which has been adapted from Poreisz [2] and Brunoni [4]. The intensity of the side effects was rated on a Likert scale from 0-5 (0 nothing at all, 1 very mild, 2 mild, 3 moderate, 4 moderate to severe, 5 severe). These side effects included: feeling unwell, headaches, changes in concentration, being sad or wanting to cry, being anxious or nervous, visual disturbances, tiredness, scalp pain, scalp tingling, scalp itching, scalp burning, feeling nausea or like throwing up, and trouble sleeping/feeling wakeful (the question 'trouble sleeping/feeling wakeful' was varied based on the time point such that children aren't going to sleep at T3,T4 or T5; therefore a question of wakefulness is more relevant; and at time points such as T1, T2, and T6 it was relevant if the child, ADHD or otherwise, had difficulty sleeping). In addition to the 13 item questionnaire, two open-ended qualitative questions were offered to address any other side effects, concerns, or notes from the participant or their parent (e.g., being tired from the night before, or if their scalp was itchy from dandruff, as well as any major changes in routine such as one participant crying the week before because their pet died). Finally, a physical assessment of the scalp was conducted before and after each tDCS session. This included checking for redness, dryness, blistering, burns, and recording if there is any pain at the site. 
These outcome measures were evaluated at multiple levels including children vs. adults, males vs. females, and controls vs. clinical based on averaged amplitudes presented in Table 1. The primary goal of this analysis was to elucidate any differences or similarities of safety/tolerability within these three grouping variables regardless of amperage. In other words, the assumption here is that tDCS side effects may be differentiated by group regardless of amplitude. However, we did report the average amplitude for these comparisons in Table 1 to demonstrate that amplitude was controlled for within these group comparisons. Following these comparisons we conducted a separate secondary analysis which collapsed the previous groups and investigated differences between side effect ratings of participants in the low $\mathrm{mA}(0-0.5 \mathrm{~mA})$ and high $\mathrm{mA}(1-2 \mathrm{~mA})$ conditions during their first tDCS session. In this case, the assumption was that different levels of current may differentiate side effect profiles. In both the primary, and secondary analyses interactions effects of the other levels were tested. The next section will provide more detail regarding the statistical approaches undertaken for these analyses.

\section{Statistical Analyses}

The plan of analyses was as follows. To determine if there were any differences in tolerability of side effects between children and adults, males and females, control and clinical, we conducted 3 repeated measure ANOVA on the 13 side effects and 6 time points as within factors, with either Age, or Sex or Clinical as between factor. The null hypothesis was that there was no difference between groups across time points (i.e., we tested the group by time interaction). Then we conducted a planned series of univariate ANOVA on each side effect at each time point with again either age, or sex or clinical as between factors. 
We used a repeated measure ANCOVA to test the effect of the amperage (low vs. high) and age (children vs. adults), and their interactions, on 13 side effects over time, with Time 2 (immediately before the first tDCS session) and Time 3 (immediately after the first tDCS session) as repeated time points, using the average side effect rating at Time 1 (one week before the first tDCS session) as covariate. Then we conducted repeated measure ANCOVA to test the effect of the amperage (low vs. high) and age (children vs. adults), and their interactions, on each side effect separately with Time 2 and Time 3 as repeated time points, using the rating of this side effect at Time 1 as covariate. We repeated the same analyses replacing age by sex (males vs. females) and by clinical (controls vs. clinical) to test the secondary hypotheses of a differential effect of amperage according to sex or clinical characteristics. Then we conducted a series of 13 analyses to test the interaction between amperage and age (or sex or clinical) for each side effect separately, at Time 2 and Time 3 separately.

We also computed the Number needed to harm (NNH) which is commonly reported in drug treatment trials [24]. $\mathrm{NNH}$ is an indicator of how many people can be exposed to a treatment before someone experiences a side effect as compared to a placebo. NNH was computed from $(\mathrm{n}=25)$ participants in the Low $\mathrm{mA}(0-0.5)$ condition and $(\mathrm{n}=35)$ participants in the High mA (1-2) condition. It was computed based on the side effects reported by these groups immediately after their first tDCS session. The total number of all side effects rated above 0 were divided by the total number of responses for each the Low and High mA conditions respectively. Then the absolute difference between those two quotients is multiplied by 100 to provide the $\mathrm{NNH}$.

The multiple analyses of variance inflated the risk and the probability of getting a significant result simply due to chance (Type I error). However, we did not present the 
Bonferroni correction in our analysis because it would have actually been in favour of our hypothesis: Basically, the best way to demonstrate that tDCS is safe and tolerable is to try and prove that it is unsafe and intolerable. If we fail at doing this then we can conclude that tDCS appears to be safe. Therefore, leaving our data uncorrected is actually a more robust test of the null hypothesis. Had we corrected our data, none of the significant differences would have survived and the safety and tolerability of tDCS may have appeared inflated.

Additionally, for every ANOVA and ANCOVA analysis, we concurrently conducted Routers Method Bayes factor independent sample tests in order to test the support for the null hypothesis.

Finally, the frequency of side effect occurrence for different groups across all side effects and time points were computed. Highlights of these findings are briefly reported in the results. The full table is available in the Appendix (Appendix B-1).

The primary objective of this study investigated the tolerability of tDCS through side effect ratings between children and adults at six different time points. Our secondary objectives were to compare the side effects in male vs female, and control vs. clinical participants. Finally we investigated if there was any main effect of high vs. low stimulation amperage immediately after the first session and one hour later, and whether this effect interacted with the three aforementioned groups. 


\section{Results}

\section{Children vs. Adults}

First, we conducted a repeated measure ANOVA comparing the pediatric group with the adult group across all 6 time points and 13 side effects. The ANOVA revealed that there was no significant difference between the age groups across time: $F(5,290)=1.719, p=.130$, partial $\eta^{2}$ $=.029$. Then we carried out 13 univariate ANOVAs, one for each side effect, for each of the 6 time points. Of these 78 comparisons, only 6 reached uncorrected significance, and 1 of these was at the one week baseline. Moreover, as shown in Figure 1, mean side effect ratings for both children and adults rarely exceeded a maximum rating of 1 'mild' in each group. What appears like random differences due to an inflated risk of Type I error were also differences between 'mild' and 'very mild' side effects, and therefore clinically insignificant. 
Figure 1. 3D bar graph of side effects at each time point for children and adults

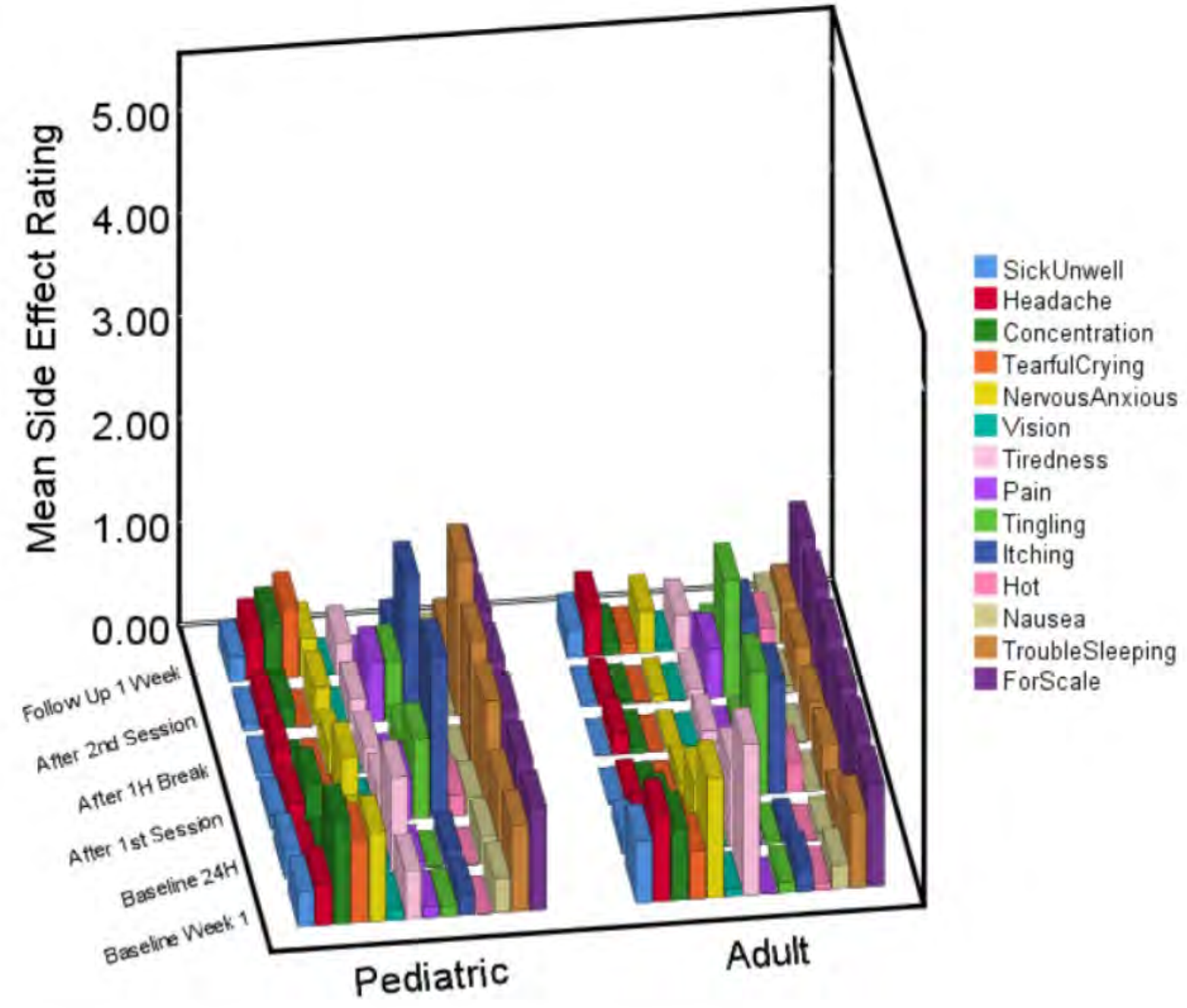

Figure 1: Average ratings for the pediatric and adult sample at each time point for the 13 different side effects. The $14^{\text {th }}$ variable is a scale at a rating of 1 , for comparison.

\section{Males vs. Females}

We conducted a second repeated measure ANOVA to compare the average side effect rating of males and females across all 6 time points and 13 side effects. We did observe a significant difference in side effects between males and females across time: $F(5,290)=2.670$, 
$p=.022$, partial $\eta^{2}=.044$. Follow-up analyses showed that this effect of sex was significant at Time 1 (one week before the tDCS session): $F(1,58)=4.873, p=.031$, partial $\eta^{2}=.077$. There were no difference between males and females in all subsequent times (all ps $>.05$ ). Next, 13 univariate ANOVAs, one for each side effect, were carried out for each of the 6 time points. Itchiness, a commonly expected side effect of tDCS, was the only 1 of 78 comparisons that was significantly different between males and females at Time 5 (after the second tDCS session). Again this significant result is mostly due to the inflated risk of Type I error, and does not survive the Bonferroni correction. Figure 2 also clearly illustrates that mean side effect ratings of both males and females rarely exceeded a maximum rating of 1 'mild'. 
Figure 2. 3D bar graph of side effects at each time point for females and males

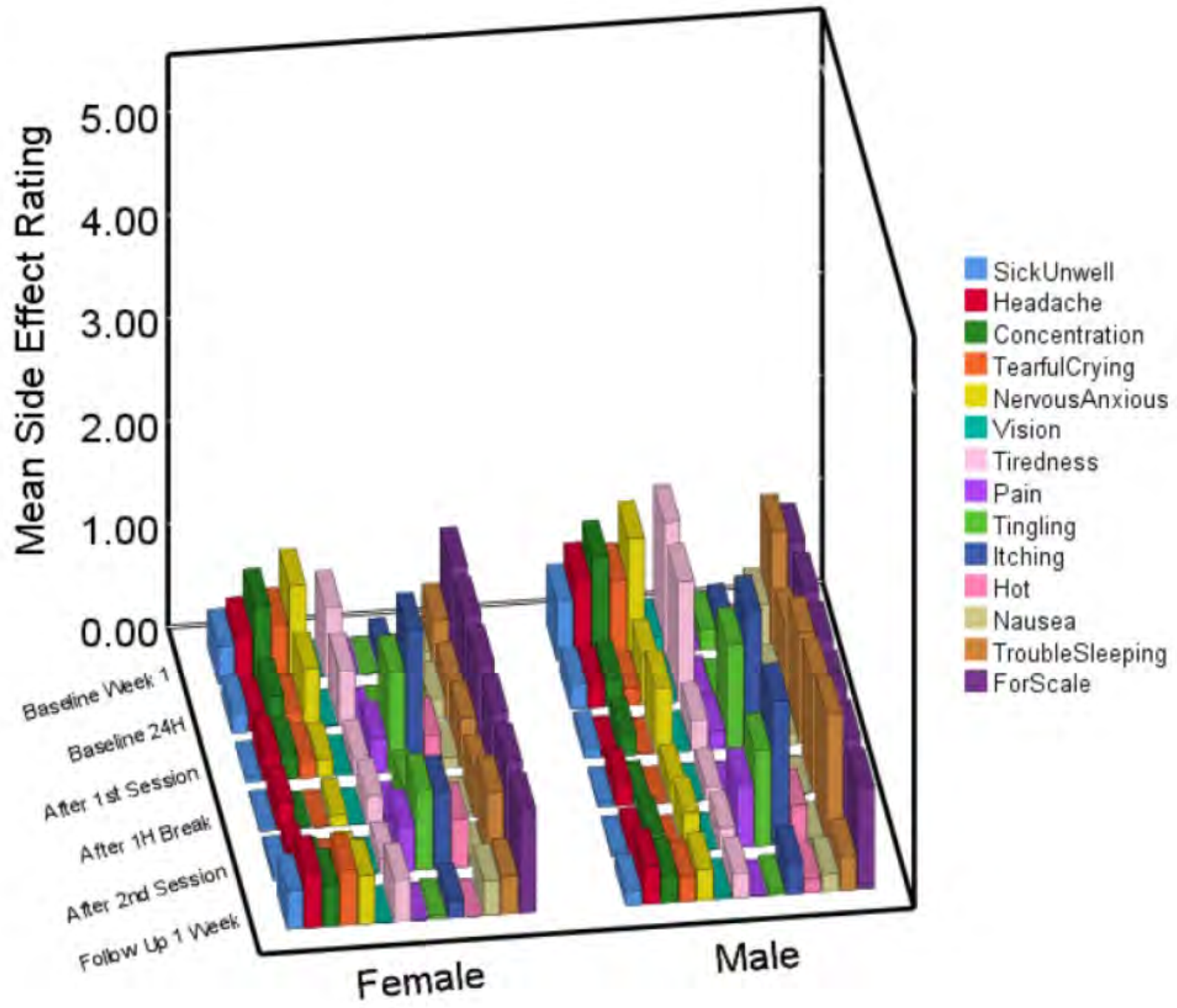

Figure 2: Average ratings for the female and male sample at each time point for the 13 different side effects. The $14^{\text {th }}$ variable is a scale at a rating of 1 , for comparison.

Controls vs. Clinical

We conducted a third repeated measure ANOVA to compare the side effect ratings of those in the clinical group and the healthy controls across all 6 time points and 13 side effects. The MANOVA did not reveal any difference between groups across time $F(5,290)=0.858, p=$ 
.510 , partial $\eta^{2}=.015$. Next, 13 univariate ANOVAs, one for each side effect, was carried out for each of the 6 time points. There were a total of 4 significant differences, one of which was concentration at the 1 week baseline. Thus, there were only 3 potential differences that could be attributed to tDCS. These 3 involved mild changes in tiredness immediately after the first session and after the 1-hour break, and in wakefulness immediately after the second sessions. Consistent with the previous 2 independent analyses, these side effect ratings also remained remarkably low (Figure 3). 
Figure 3. 3D bar graph of side effects at each time point for healthy and clinical

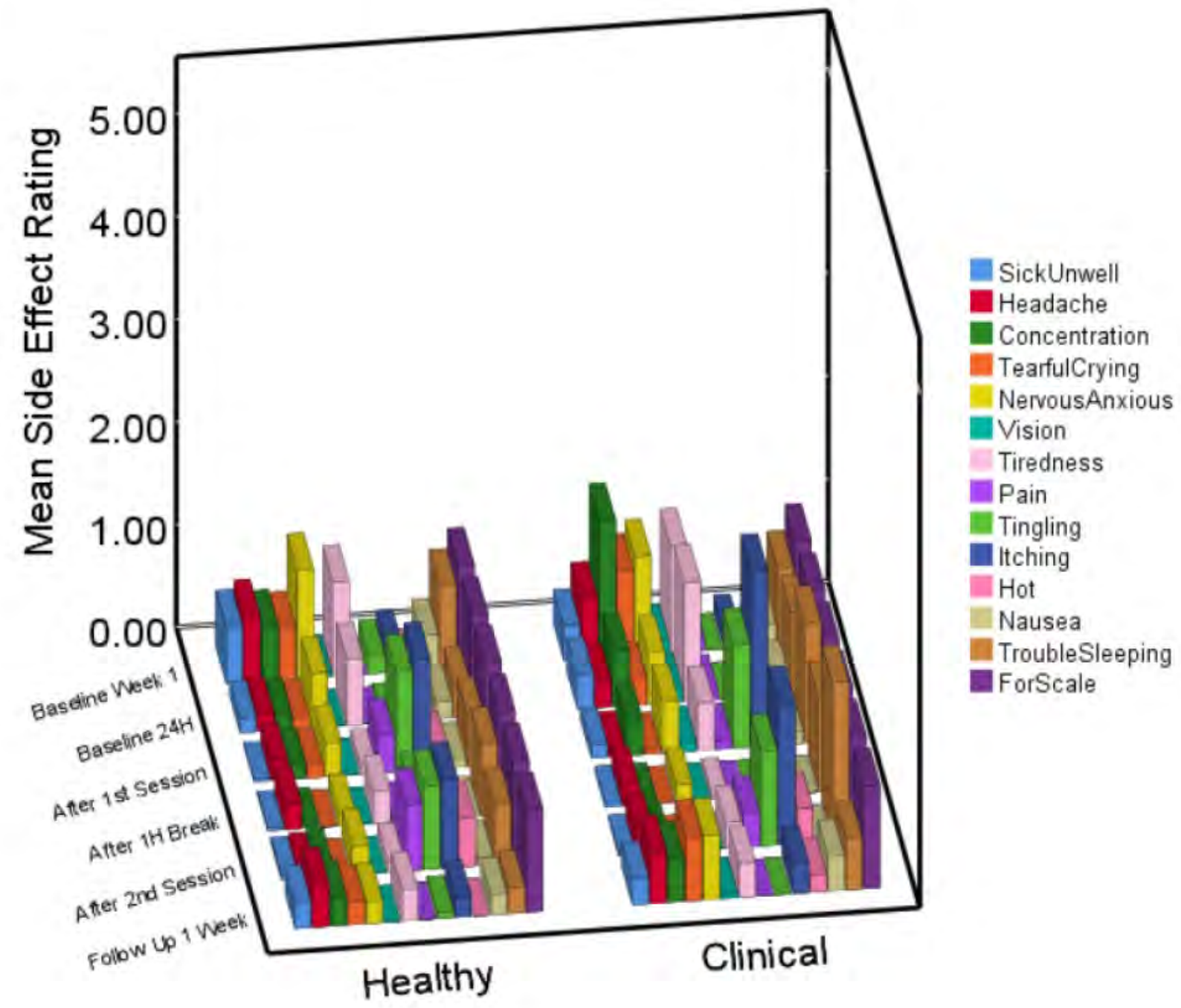

Figure 3: Average ratings for the control and the clinical sample at each time point for the 13 different side effects. The $14^{\text {th }}$ variable is a scale at a rating of 1 , for comparison.

Table 4 presents the 234 (13X6X3) comparisons between 3 groups of 13 side effects for each of the six time points. A total of 11 significant differences were present across all 234 comparisons (.047\%), which is less than expected even by chance (e.g., alpha .05). These 
differences also did not appear to cluster on any specific side effect or time point but rather appeared quite randomly. No group difference survived a multiple comparison correction.

Table 4. F statistics of each group effect and associated partial $\eta$, as well as Bayes factor

\begin{tabular}{|c|c|c|c|c|c|c|c|c|c|c|}
\hline & & \multicolumn{3}{|c|}{ Age } & \multicolumn{3}{|c|}{ Sex } & \multicolumn{3}{|c|}{ Clinical } \\
\hline Side effect & Time & $\mathrm{F}_{1,58}$ & $\eta_{\text {partial }}$ & Bayes & $\mathrm{F}_{1,58}$ & $\eta_{\text {partial }}$ & Bayes & $\mathrm{F}_{1,58}$ & $\eta_{\text {partial }}$ & Bayes \\
\hline \multirow[t]{6}{*}{ Sick/Unwell } & $\mathrm{T} 1$ & 1.193 & 0.020 & 3.000 & 0.665 & 0.011 & 3.803 & 0.790 & 0.013 & 3.534 \\
\hline & $\mathrm{T} 2$ & 0.310 & 0.005 & 4.470 & 0.034 & 0.001 & 5.065 & 1.143 & 0.019 & 3.017 \\
\hline & T3 & 1.634 & 0.027 & 2.460 & 0.400 & 0.007 & 4.288 & 0.823 & 0.014 & 3.483 \\
\hline & $\mathrm{T} 4$ & 2.071 & 0.034 & 2.030 & 0.000 & 0.000 & 5.144 & 0.083 & 0.001 & 4.863 \\
\hline & T5 & 0.341 & 0.006 & 4.400 & 0.341 & 0.006 & 4.404 & 0.057 & 0.001 & 4.923 \\
\hline & T6 & 0.026 & 0.000 & 5.080 & 1.300 & 0.022 & 2.858 & 0.000 & 0.000 & 5.051 \\
\hline \multirow[t]{6}{*}{ Headache } & T1 & 3.180 & 0.052 & 1.245 & 1.482 & 0.025 & 2.635 & 0.048 & 0.001 & 4.943 \\
\hline & $\mathrm{T} 2$ & 0.164 & 0.003 & 4.773 & 0.164 & 0.003 & 4.773 & 0.247 & 0.004 & 4.516 \\
\hline & T3 & 1.402 & 0.024 & 2.730 & 2.814 & 0.046 & 1.462 & 0.590 & 0.010 & 3.867 \\
\hline & T4 & 0.171 & 0.003 & 4.760 & 0.171 & 0.003 & 4.760 & 0.064 & 0.001 & 4.907 \\
\hline & T5 & 0.644 & 0.011 & 3.839 & 0.644 & 0.011 & 3.839 & 1.751 & 0.029 & 2.301 \\
\hline & T6 & 0.015 & 0.000 & 5.110 & 0.375 & 0.006 & 4.337 & 0.090 & 0.002 & 4.850 \\
\hline \multirow{6}{*}{$\begin{array}{l}\text { Difficulty } \\
\text { Concentrating }\end{array}$} & $\mathrm{T} 1$ & 0.751 & 0.013 & 3.659 & 0.452 & 0.008 & 4.189 & 5.628 & 0.088 & 0.432 \\
\hline & $\mathrm{T} 2$ & 0.829 & 0.014 & 3.533 & 0.091 & 0.002 & 4.936 & 3.365 & 0.055 & 1.134 \\
\hline & T3 & 4.840 & 0.077 & 0.609 & 0.037 & 0.001 & 5.058 & 1.232 & 0.021 & 2.900 \\
\hline & $\mathrm{T} 4$ & 2.071 & 0.034 & 2.027 & 2.071 & 0.034 & 2.027 & 0.083 & 0.001 & 4.863 \\
\hline & $\mathrm{T} 5$ & 2.054 & 0.034 & 2.043 & 2.054 & 0.034 & 2.043 & 0.013 & 0.000 & 5.021 \\
\hline & T6 & 2.630 & 0.043 & 1.585 & 0.281 & 0.005 & 4.527 & 0.293 & 0.005 & 4.423 \\
\hline \multirow[t]{6}{*}{ Tearful/Crying } & T1 & 1.121 & 0.019 & 3.098 & 0.398 & 0.007 & 4.291 & 1.075 & 0.018 & 3.110 \\
\hline & $\mathrm{T} 2$ & 0.000 & 0.000 & 5.144 & 0.000 & 0.000 & 5.144 & 0.730 & 0.012 & 3.630 \\
\hline & T3 & 1.032 & 0.017 & 3.224 & 2.959 & 0.049 & 1.372 & 0.473 & 0.008 & 4.077 \\
\hline & $\mathrm{T} 4$ & & & & & & 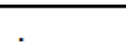 & & 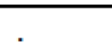 & 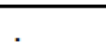 \\
\hline & $\mathrm{T} 5$ & & & & & & 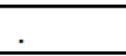 & & & $\sigma_{2}$ \\
\hline & T6 & 5.046 & 0.080 & 0.558 & 1.331 & 0.022 & 2.819 & 2.342 & 0.039 & 1.772 \\
\hline \multirow[t]{6}{*}{ Nervous/Anxious } & T1 & 0.802 & 0.014 & 3.575 & 0.483 & 0.008 & 4.131 & 0.080 & 0.001 & 4.871 \\
\hline & $\mathrm{T} 2$ & 0.738 & 0.013 & 3.680 & 0.134 & 0.002 & 4.839 & 0.010 & 0.000 & 5.029 \\
\hline & T3 & 3.856 & 0.062 & 0.929 & 3.856 & 0.062 & 0.929 & 0.446 & 0.008 & 4.126 \\
\hline & $\mathrm{T} 4$ & 2.814 & 0.046 & 1.462 & 0.497 & 0.008 & 4.104 & 0.037 & 0.001 & 4.968 \\
\hline & T5 & 2.918 & 0.048 & 1.396 & 2.918 & 0.048 & 1.396 & 0.700 & 0.012 & 3.680 \\
\hline & T6 & 0.015 & 0.000 & 5.109 & 0.379 & 0.006 & 4.329 & 2.191 & 0.036 & 1.895 \\
\hline \multirow[t]{6}{*}{ Vision } & $\mathrm{T} 1$ & 0.077 & 0.001 & 4.967 & 1.986 & 0.033 & 2.105 & 0.322 & 0.006 & 4.365 \\
\hline & $\mathrm{T} 2$ & 1.000 & 0.017 & 3.270 & 1.000 & 0.017 & 3.270 & 1.513 & 0.025 & 2.558 \\
\hline & T3 & 2.071 & 0.034 & 2.027 & 0.000 & 0.000 & 5.144 & 3.164 & 0.052 & 1.238 \\
\hline & $\mathrm{T} 4$ & 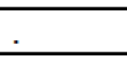 & . & 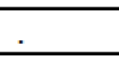 & . & . & . & . & . & . \\
\hline & T5 & & & &. & & . & . & . & . \\
\hline & T6 & 1.000 & 0.017 & 3.270 & 1.000 & 0.017 & 3.270 & 1.513 & 0.025 & 2.558 \\
\hline \multirow[t]{3}{*}{ Tiredness } & T1 & 11.308 & 0.163 & 0.044 & 3.619 & 0.059 & 1.029 & 0.142 & 0.002 & 4.735 \\
\hline & $\mathrm{T} 2$ & 0.086 & 0.001 & 4.947 & 3.649 & 0.059 & 1.016 & 2.383 & 0.039 & 1.741 \\
\hline & T3 & 0.632 & 0.011 & 3.860 & 0.632 & 0.011 & 3.860 & 5.172 & 0.082 & 0.523 \\
\hline
\end{tabular}




\begin{tabular}{|c|c|c|c|c|c|c|c|c|c|c|}
\hline & T4 & 0.286 & 0.005 & 4.517 & 1.160 & 0.020 & 3.043 & 4.617 & 0.074 & 0.662 \\
\hline & $\mathrm{T} 5$ & 0.771 & 0.013 & 3.626 & 0.191 & 0.003 & 4.716 & 0.803 & 0.014 & 3.513 \\
\hline & T6 & 0.022 & 0.000 & 5.092 & 0.568 & 0.010 & 3.974 & 0.015 & 0.000 & 5.017 \\
\hline \multirow[t]{6}{*}{ Pain } & T1 & 1.000 & 0.017 & 3.270 & 1.000 & 0.017 & 3.270 & 1.513 & 0.025 & 2.558 \\
\hline & $\mathrm{T} 2$ & 1.000 & 0.017 & 3.270 & 1.000 & 0.017 & 3.270 & 1.513 & 0.025 & 2.558 \\
\hline & T3 & 3.575 & 0.058 & 1.049 & 1.058 & 0.018 & 3.185 & 2.911 & 0.048 & 1.382 \\
\hline & $\mathrm{T} 4$ & 1.000 & 0.017 & 3.270 & 1.000 & 0.017 & 3.270 & 0.663 & 0.011 & 3.742 \\
\hline & $\mathrm{T} 5$ & 0.148 & 0.003 & 4.809 & 0.413 & 0.007 & 4.264 & 1.353 & 0.023 & 2.747 \\
\hline & T6 & & & & & & & & & \\
\hline \multirow[t]{6}{*}{ Tingling } & T1 & 0.000 & 0.000 & 5.144 & 3.222 & 0.053 & 1.223 & 2.109 & 0.035 & 1.964 \\
\hline & $\mathrm{T} 2$ & 1.000 & 0.017 & 3.270 & 1.000 & 0.017 & 3.270 & 0.663 & 0.011 & 3.742 \\
\hline & T3 & 3.016 & 0.049 & 1.338 & 0.017 & 0.000 & 5.105 & 0.011 & 0.000 & 5.025 \\
\hline & $\mathrm{T} 4$ & 0.910 & 0.015 & 3.406 & 3.055 & 0.050 & 1.316 & 3.041 & 0.050 & 1.305 \\
\hline & T5 & 3.636 & 0.059 & 1.022 & 0.297 & 0.005 & 4.493 & 0.127 & 0.002 & 4.769 \\
\hline & T6 & 2.071 & 0.034 & 2.027 & 0.000 & 0.000 & 5.144 & 1.365 & 0.023 & 2.732 \\
\hline \multirow[t]{6}{*}{ Itching } & T1 & 0.079 & 0.001 & 4.961 & 0.723 & 0.012 & 3.705 & 0.480 & 0.008 & 4.064 \\
\hline & $\mathrm{T} 2$ & 0.867 & 0.015 & 3.472 & 0.867 & 0.015 & 3.472 & 0.254 & 0.004 & 4.501 \\
\hline & T3 & 1.661 & 0.028 & 2.433 & 0.033 & 0.001 & 5.068 & 2.921 & 0.048 & 1.376 \\
\hline & T4 & 0.436 & 0.007 & 4.219 & 1.785 & 0.030 & 2.302 & 0.290 & 0.005 & 4.428 \\
\hline & T5 & 3.251 & 0.053 & 1.207 & 4.067 & 0.066 & 0.848 & 2.252 & 0.037 & 1.844 \\
\hline & T6 & 1.919 & 0.032 & 2.169 & 0.838 & 0.014 & 3.518 & 0.424 & 0.007 & 4.167 \\
\hline \multirow[t]{6}{*}{ Hot } & T1 & 1.000 & 0.017 & 3.270 & 1.000 & 0.017 & 3.270 & 0.663 & 0.011 & 3.742 \\
\hline & $\mathrm{T} 2$ & & & & & & & & & \\
\hline & T3 & 0.118 & 0.002 & 4.874 & 1.083 & 0.018 & 3.150 & 0.242 & 0.004 & 4.525 \\
\hline & $\mathrm{T} 4$ & 1.000 & 0.017 & 3.270 & 1.000 & 0.017 & 3.270 & 1.513 & 0.025 & 2.558 \\
\hline & $\mathrm{T} 5$ & 4.568 & 0.073 & 0.684 & 0.226 & 0.004 & 4.640 & 0.421 & 0.007 & 4.174 \\
\hline & T6 & 1.000 & 0.017 & 3.270 & 1.000 & 0.017 & 3.270 & 1.513 & 0.025 & 2.558 \\
\hline \multirow[t]{6}{*}{ Nausea } & T1 & 0.198 & 0.003 & 4.701 & 1.092 & 0.018 & 3.137 & 0.297 & 0.005 & 4.415 \\
\hline & $\mathrm{T} 2$ & 1.601 & 0.027 & 2.498 & 0.174 & 0.003 & 4.753 & 0.000 & 0.000 & 5.051 \\
\hline & T3 & 1.389 & 0.023 & 2.746 & 0.000 & 0.000 & 5.144 & 0.127 & 0.002 & 4.767 \\
\hline & $\mathrm{T} 4$ & 1.000 & 0.017 & 3.270 & 1.000 & 0.017 & 3.270 & 0.663 & 0.011 & 3.742 \\
\hline & T5 & 0.341 & 0.006 & 4.404 & 0.341 & 0.006 & 4.404 & 2.109 & 0.035 & 1.964 \\
\hline & T6 & 0.564 & 0.010 & 3.981 & 0.564 & 0.010 & 3.981 & 0.375 & 0.006 & 4.262 \\
\hline \multirow{6}{*}{$\begin{array}{l}\text { Trouble } \\
\text { Sleeping/ } \\
\text { Wakefulness }\end{array}$} & $\mathrm{T} 1$ & 0.089 & 0.002 & 4.940 & 3.786 & 0.061 & 0.957 & 0.026 & 0.000 & 4.991 \\
\hline & $\mathrm{T} 2$ & 2.397 & 0.040 & 1.756 & 1.124 & 0.019 & 3.092 & 2.582 & 0.043 & 1.596 \\
\hline & T3 & 3.168 & 0.052 & 1.252 & 2.539 & 0.042 & 1.649 & 3.034 & 0.050 & 1.309 \\
\hline & $\mathrm{T} 4$ & 4.262 & 0.068 & 0.780 & 2.943 & 0.048 & 1.381 & 0.272 & 0.005 & 4.464 \\
\hline & T5 & 7.340 & 0.112 & 0.214 & 1.676 & 0.028 & 2.417 & 5.530 & 0.087 & 0.450 \\
\hline & T6 & 0.141 & 0.002 & 4.823 & 0.016 & 0.000 & 5.108 & 0.859 & 0.015 & 3.426 \\
\hline
\end{tabular}

Significant effects are in bold; . indicates that all the ratings were zero.

\section{Bayesian approach}

Conversely, on average these group comparisons have an average Bayes factor of 3.31

(standard deviation $=1.40$ ) which demonstrates moderate support for the null hypothesis. For the 
comparison between children and adults the Bayes factor is 3.09 (standard deviation=1.47), for the comparison between males and females 3.45 (standard deviation=1.31), and for the comparison between the control and the clinical group 3.39 (standard deviation=1.40). The boxplots of the distributions of the Bayes statistic is presented in Figure 4.

Figure 4. Boxplot of Bayesian factor average for age sex and clinical status

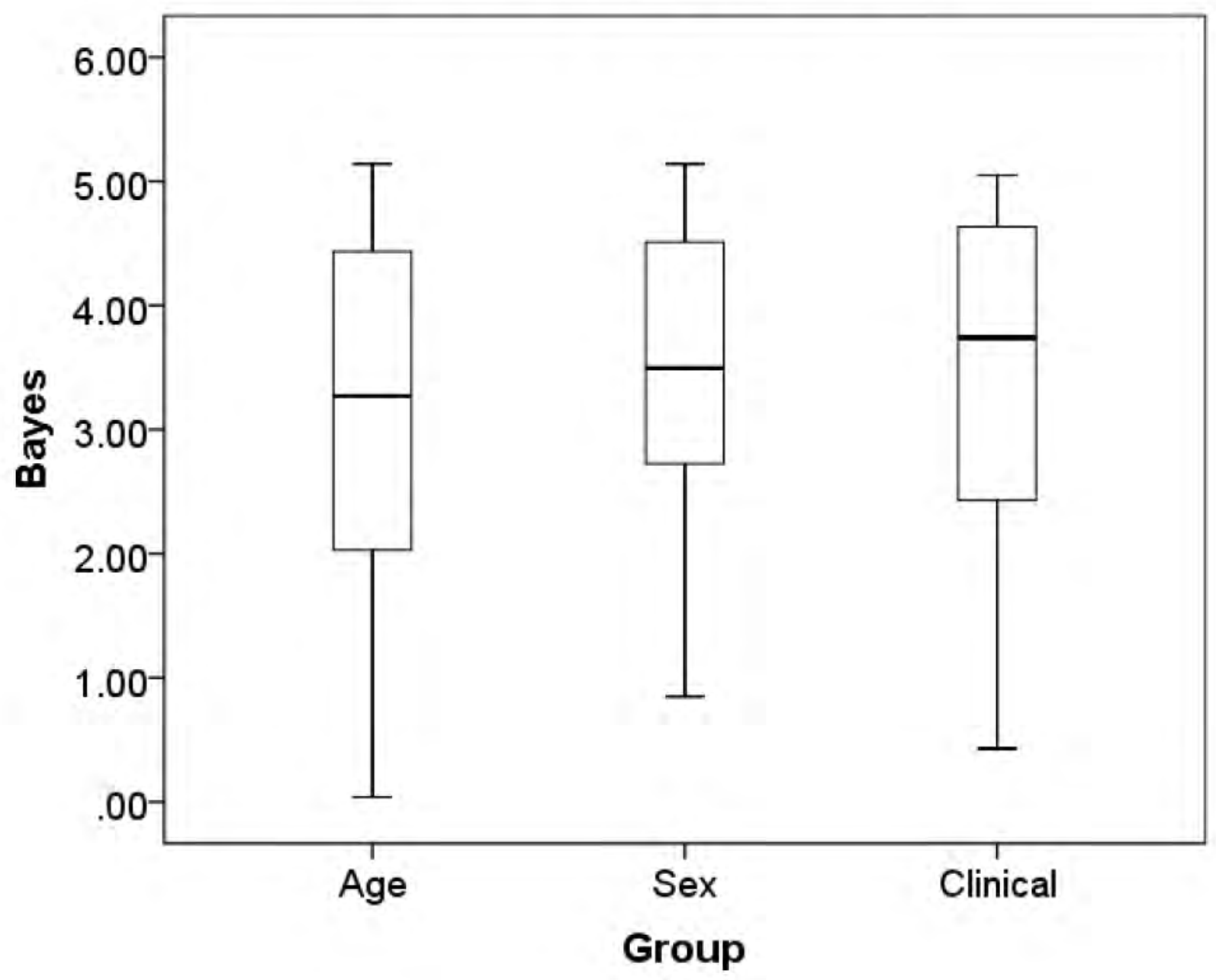

Figure 4: Boxplots of the distribution of the Bayes factor for each group difference in the ratings across the 6 times and 13 side effects. 


\section{Low vs. High Amperage}

In a repeated measure ANCOVA using Amperage (Low vs. High) as between factors and the 13 side effects at Time 2 and Time 3 as within factors repeated measures, and the average side effect rating at Time 1 as covariate, there was no significant effect of low vs. high amperage on side effect ratings from pre to post tDCS. Additionally, the amperage by age interaction was not significant: $F(1,55)=0.461, p=.500$, partial $\eta^{2}=.008$. When examined for each side effect separately, with Time 2 and Time 3 as within factors, and the side effect rating at Time 1 as covariate, none of these age by amperage interactions were significant (all $p \mathrm{~s}>.05$ ). The age by amperage interactions were also never significant when each side effect was examined at each time (T2, T3, T4) separately using the measure at $\mathrm{T} 1$ as covariate (all $p s>.05)$.

When age was replaced by sex in the previous analyses, the amperage by sex interaction was not significant: $F(1,55)=0.090, p=.765$, partial $\eta 2=.002$. When examined for each side effect separately, with Time 2 and Time 3 as within factors, and the side effect rating at Time 1 as covariate, none of these age by amperage interactions were significant (all ps $>.05$ ). The age by amperage interactions was also never significant when each side effect was examined at each time (T2, T3, T4) separately using the measure at $\mathrm{T} 1$ as covariate (all ps $>.05)$.

When clinical status was used in the previous analyses instead of age, the amperage by clinical was not significant: $F(1,55)=0.473, p=.494$, partial $\eta 2=.009$. When examined for each side effect separately, with Time 2 and Time 3 as within factors, and the side effect rating at Time 1 as covariate, only one age by amperage interactions was significant for the side effect "hot": $F(1,55)=4.689, \mathrm{p}=.035$, partial $\eta 2=.079$. When each side effect was examined at each time (T2, T3, T4) separately using the measure at $\mathrm{T} 1$ as covariate, the age by amperage 
interactions was significant for "hot" at Time 3, after the first session: $F(1,55)=4.689, \mathrm{p}=$ .035 , partial $\eta 2=.079$.

Given the lack of interaction with amperage, we tested this between factor across the whole sample at each time (Time 2, 3,4) and for each side effect. Only one significant difference emerged for "itching" but before the first tDCS session. All these results are presented in Table 5. We observed only 2 significant effects that are likely due to Type I errors due to multiple testing. These comparisons also demonstrate moderate support for the null hypothesis with an average Bayes factor of 3.41. Finally, we computed the Number Needed to Harm (NNH) which was 60 . This means that 1 in every 60 people who use this tDCS may experience some side effect greater than a rating of 0 when receiving a current of 1 or $2 \mathrm{~mA}$, as compared to 0 or 0.5 $\mathrm{mA}$.

Table 5: Effect of Low vs. High amperage across the whole sample, and interactions with age, sex and clinical status

\begin{tabular}{|l|l|l|l|l|l|l|l|l|l|l|}
\hline & & \multicolumn{3}{|c|}{ Amperage } & \multicolumn{2}{c|}{ Age X Amp } & \multicolumn{2}{c|}{ Sex X Amp } & \multicolumn{2}{c|}{$\begin{array}{c}\text { Clinical X } \\
\text { Amp }\end{array}$} \\
\hline Side effect & Time & $\mathrm{F}_{1,57}$ & $\eta_{\text {partial }}$ & Bayes & $\mathrm{F}_{1,55}$ & $\eta_{\text {partial }}$ & $\mathrm{F}_{1,55}$ & $\eta_{\text {partial }}$ & $\mathrm{F}_{1,55}$ & $\eta_{\text {partial }}$ \\
\hline \multirow{4}{*}{ Sick Unwell } & $\mathrm{T} 2$ & 0.057 & 0.001 & 4.322 & 0.056 & 0.001 & 3.695 & 0.063 & 3.191 & 0.055 \\
\cline { 2 - 12 } & $\mathrm{T} 3$ & 1.167 & 0.020 & 3.012 & 0.970 & 0.017 & 0.146 & 0.003 & 0.586 & 0.011 \\
\cline { 2 - 12 } & $\mathrm{T} 4$ & 1.733 & 0.030 & 2.626 & 0.594 & 0.011 & 0.017 & 0.000 & 0.072 & 0.001 \\
\hline \multirow{4}{*}{ Headache } & $\mathrm{T} 2$ & 2.280 & 0.038 & 1.439 & 1.660 & 0.029 & 0.238 & 0.004 & 0.271 & 0.005 \\
\cline { 2 - 12 } & $\mathrm{T} 3$ & 0.339 & 0.006 & 4.325 & 0.151 & 0.003 & 0.418 & 0.008 & 0.419 & 0.008 \\
\cline { 2 - 12 } & $\mathrm{T} 4$ & 0.558 & 0.010 & 3.485 & 2.917 & 0.050 & 0.004 & 0.000 & 0.100 & 0.002 \\
\hline \multirow{4}{*}{$\begin{array}{l}\text { Concentrating } \\
\text { Crying }\end{array}$} & $\mathrm{T} 2$ & 0.056 & 0.001 & 5.026 & 0.030 & 0.001 & 0.304 & 0.006 & 0.002 & 0.000 \\
\cline { 2 - 11 } & $\mathrm{T} 3$ & 0.901 & 0.016 & 3.380 & 1.610 & 0.028 & 0.002 & 0.000 & 0.001 & 0.000 \\
\cline { 2 - 11 } & $\mathrm{T} 4$ & 0.062 & 0.001 & 4.949 & 0.003 & 0.000 & 0.203 & 0.004 & 1.370 & 0.024 \\
\cline { 2 - 11 } & $\mathrm{T} 2$ & 0.027 & 0.000 & 3.461 & 1.154 & 0.021 & 0.003 & 0.000 & 0.076 & 0.001 \\
\cline { 2 - 11 } & $\mathrm{T} 3$ & 3.575 & 0.059 & 0.784 & 1.383 & 0.025 & 3.156 & 0.054 & 0.733 & 0.013 \\
\hline \multirow{2}{*}{$\begin{array}{l}\text { Nervous } \\
\text { Anxious }\end{array}$} & $\mathrm{T} 2$ & 0.371 & 0.006 & 1.287 & 3.937 & 0.067 & 0.071 & 0.001 & 0.408 & 0.007 \\
\cline { 2 - 10 } & $\mathrm{T} 3$ & 0.236 & 0.004 & 2.903 & 0.107 & 0.002 & 0.396 & 0.007 & 0.032 & 0.001 \\
\cline { 2 - 10 } & $\mathrm{T} 4$ & 0.049 & 0.001 & 4.795 & 0.472 & 0.009 & 1.383 & 0.025 & 0.374 & 0.007 \\
\hline Vision & $\mathrm{T} 2$ & 0.057 & 0.001 & 3.682 & 1.351 & 0.024 & 0.045 & 0.001 & 1.287 & 0.023 \\
\hline
\end{tabular}




\begin{tabular}{|c|c|c|c|c|c|c|c|c|c|c|}
\hline & T3 & 0.040 & 0.001 & 4.949 & 0.079 & 0.001 & 1.510 & 0.027 & 0.074 & 0.001 \\
\hline & $\mathrm{T} 4$ & . & . & 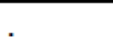 & . & . & . & . & . & . \\
\hline \multirow[t]{3}{*}{ Tiredness } & $\mathrm{T} 2$ & 1.701 & 0.029 & 3.093 & 1.736 & 0.031 & 2.543 & 0.044 & 0.203 & 0.004 \\
\hline & T3 & 0.008 & 0.000 & 5.070 & 1.042 & 0.019 & 0.198 & 0.004 & 0.152 & 0.003 \\
\hline & T4 & 1.131 & 0.019 & 2.964 & 1.965 & 0.034 & 0.037 & 0.001 & 1.890 & 0.033 \\
\hline \multirow[t]{3}{*}{ Pain } & $\mathrm{T} 2$ & . & . & 3.682 & 0.000 & 0.000 & . & . & 0.000 & 0.000 \\
\hline & T3 & 0.315 & 0.006 & 4.496 & 0.085 & 0.002 & 0.004 & 0.000 & 0.295 & 0.005 \\
\hline & $\mathrm{T} 4$ & 1.369 & 0.023 & 2.691 & 1.269 & 0.023 & 0.916 & 0.016 & 0.899 & 0.016 \\
\hline \multirow[t]{3}{*}{ Tingling } & $\mathrm{T} 2$ & 0.806 & 0.014 & 3.682 & 2.960 & 0.051 & 0.692 & 0.012 & 0.612 & 0.011 \\
\hline & T3 & 0.809 & 0.014 & 3.366 & 0.502 & 0.009 & 0.839 & 0.015 & 1.683 & 0.030 \\
\hline & $\mathrm{T} 4$ & 0.724 & 0.013 & 3.421 & 0.109 & 0.002 & 0.008 & 0.000 & 0.450 & 0.008 \\
\hline \multirow[t]{3}{*}{ Itching } & $\mathrm{T} 2$ & 5.470 & 0.088 & 2.619 & 0.148 & 0.003 & 1.780 & 0.031 & 1.414 & 0.025 \\
\hline & T3 & 2.228 & 0.038 & 2.712 & 0.792 & 0.014 & 0.000 & 0.000 & 0.773 & 0.014 \\
\hline & T4 & 2.034 & 0.034 & 1.147 & 0.234 & 0.004 & 1.243 & 0.022 & 0.076 & 0.001 \\
\hline \multirow[t]{3}{*}{ Hot } & $\mathrm{T} 2$ & . & . & . & . & . & . & . & . & . \\
\hline & T3 & 0.011 & 0.000 & 5.072 & 0.142 & 0.003 & 0.654 & 0.012 & 4.689 & 0.079 \\
\hline & $\mathrm{T} 4$ & 1.470 & 0.025 & 2.691 & 1.483 & 0.026 & 0.889 & 0.016 & 2.104 & 0.037 \\
\hline \multirow[t]{3}{*}{ Nausea } & $\mathrm{T} 2$ & 0.395 & 0.007 & 4.548 & 0.569 & 0.010 & 0.930 & 0.017 & 0.571 & 0.010 \\
\hline & T3 & 0.104 & 0.002 & 4.882 & 0.571 & 0.010 & 0.994 & 0.018 & 1.370 & 0.024 \\
\hline & $\mathrm{T} 4$ & 1.093 & 0.019 & 3.682 & 0.002 & 0.000 & 0.466 & 0.008 & 1.341 & 0.024 \\
\hline \multirow{3}{*}{$\begin{array}{l}\text { Trouble } \\
\text { Sleeping/ } \\
\text { Wakefulness }\end{array}$} & $\mathrm{T} 2$ & 0.689 & 0.012 & 4.085 & 0.263 & 0.005 & 1.636 & 0.029 & 0.010 & 0.000 \\
\hline & T3 & 1.625 & 0.028 & 2.438 & 1.408 & 0.025 & 0.148 & 0.003 & 0.135 & 0.002 \\
\hline & $\mathrm{T} 4$ & 1.984 & 0.034 & 2.078 & 0.157 & 0.003 & 0.006 & 0.000 & 0.159 & 0.003 \\
\hline
\end{tabular}

Significant effects are in bold; . indicates that all the ratings were zero.

\section{Assessment of Skin and Scalp}

Furthermore, physical assessment of skin and scalp revealed that neither children nor adults experienced any dryness $(0 \%)$, blistering $(0 \%)$, or burns $(0 \%)$ out of 120 total sessions. However, redness was seen under the electrodes in $8.3 \%$ of 60 sessions in children, and $18.3 \%$ of the 60 sessions in adults. There were no adverse events or drop outs.

\section{Frequency of Side Effects}

Most of the participants reported no side effect. However, in the week leading up to tDCS $36.7 \%$ of children reported some degree of concentration problems, and $30 \%$ of them tiredness, 
trouble sleeping/wakefulness, and anxiousness. Some level of tiredness was reported by $70 \%$ of adults, anxiousness by $60 \%$, headache by $43.3 \%$, and concentration problems by $40 \%$. Immediately after the first session, $63.3 \%$ of children reported some itchiness and $50 \%$ tingling. At the same time point, $53.3 \%$ of adults reported some itchiness and $70 \%$ of them some tingling. Also after the first session two children reported severe wakefulness, and two adults reported moderate to severe wakefulness. After a one hour break side effects were mostly extinguished including wakefulness in adults, but now six children reported severe wakefulness (5 males/1 female, $3 \mathrm{ADHD} / 3$ neurotypical, 5 DLPFC/1 Temporal Cortex, 5 active (1-2ma)/1 sham). Consistent with the first session, $56.7 \%$ of children reported some itching following the second session and $56.7 \%$ of adults some tingling. In the week following $t D C S 20 \%$ of adults reported some headache, compared to $16.7 \%$ for children; though this may not be due to tDCS as it is actually $23.3 \%$ and $13.3 \%$ less than each group's one week baseline measurement. The effects on wakefulness also appear to have dissipated in the week following tDCS.

\section{Discussion}

The present study provided evidence for the short term safety and tolerability of tDCS by examining side effects of 120 sessions between youth and adults. From all the side effect ratings at different time points after tDCS (T3-T6), 84.2\% were zero. In addition, no participants dropped out indicating $100 \%$ acceptability. The average side effect rating was below 1 'very mild' on a 5-point Likert scale. Overall, child and adult side effect ratings did not appear to differ, and the few differences we observed can be accounted for by an inflated risk of Type I error due to multiple testing. When applying a correction for multiple testing, none of these differences survived. 
Currently, a clear gap exists between the clinical application of tDCS in adults and children as $95 \%$ of the published tDCS research has been conducted in adults [8]. Therefore, in order to establish the safety and tolerability of tDCS in children, we conducted the first prospective randomized double-blind sham-controlled trial which specifically examined side effects and adverse events related to tDCS between children and adults. We randomized the electrode location and the amperage applied in 2 tDCS sessions of 10 minutes separated by a pause of 1 hour. It should be noted that since each participant received a different protocol in session 1 and session 2 our analysis of side effects in relation to tDCS amperage was limited to the first session.

We evaluated the more commonly reported side effects one week before tDCS, before and after each tDCS session and one week after the session. Our design further allowed a direct comparison of safety and tolerability between children and adults, as well as between males and females and clinically referred vs. healthy control participants. There were no significant differences within these groups. We also included a direct analysis of stimulation amperage on side effects, an outcome that has yet to be clearly reported in children. The stimulation amperage did not affect differentially the reported side effect as a function of sex, or clinical status. This information is important for the safety of tDCS whatever age, sex or clinical status.

One could object that we did not have enough power to detect very small effects, which is certainly true. Based on our data, a power analysis shows that in order to have an $80 \%$ chance of detecting an age effect size as small as 0.03 (average partial $\eta$ ) across the 6 measures with an alpha of $5 \%$, the projected sample size is close to 6000 subjects. Rather, our objective was to estimate the effect size of the tDCS effect and how it differs with age, sex and clinical status. In order to illustrate this effect size clinically, a meaningful number is the Number Needed to Harm 
[25]. In these tDCS sessions the NNH was 60 . This means that a clinician should treat 60 patients with a similar tDCS protocol to be likely to have 1 patient reporting a side effect greater than a rating of 0 for a high amperage ( 1 or $2 \mathrm{~mA}$ ) as compared to a sham or low amperage $(0.5$ $\mathrm{mA}$ ). These side effects would be most likely itchiness, tingling, and sleeping problems. It is meaningful to compare this NNH for tDCS with those obtained for frequently used medication. For example, methylphenidate, the most common drug used for ADHD, has an $\mathrm{NNH}$ of 4 for appetite, 7 for wakefulness/insomnia, 9 for stomach aches, 10 for drowsiness, and 11 for dizziness in a systematic review of nearly 3000 children [26]. Therefore, based on this analysis tDCS appears about 10 times less harmful than the presently most commonly prescribed medication for ADHD. Nonetheless, our sample size was $1 / 50$ that of the review from Schachter et al. [26] and accordingly future research will have to further validate the NNH for tDCS in a larger sample,

Since the side effects of tDCS are so mild, and the likelihood of experiencing them are much lower, it seems that tDCS should be considered more seriously as an adjunct or alternative treatment option to medication. That being said, the present study was limited to providing only 2 tDCS sessions per participant; in clinical practice it is expected that upwards of $20 \mathrm{tDCS}$ sessions will be required over the course of 4 weeks. To that end, strong efficacy and long term safety trials exploring the full tDCS treatment dosage for ADHD will be critically important for future research. This is in line with our recent qualitative study that showed how tDCS is raising hope for parents of children with ADHD dealing with pharmacological treatment: These parents made it clear that the safety, tolerability and side effects of tDCS were imperitave for accepting a treatment, provided that it is effective, relatively inexpensive, practical and accessible [27]. 


\section{Conclusions}

This study demonstrated evidence supporting the overall short term safety and tolerability of tDCS, including electrode placement and current up to $2 \mathrm{~mA}$, in children/adults, males/females, and healthy/clinical populations. These results of the present study should help to facilitate the clinical uptake and transferability of adult tDCS evidence to youth applications, as well as the development of child specific protocols. These results are concordant with current safety evidence and extend them in healthy children and children with ADHD.

\section{Funding Statement}

This research did not receive any specific grant from funding agencies in the public, commercial, or not-for-profit sectors. 


\section{References}

1. Nitsche, M.; Liebetanz, D.; Lang, N.; Antal, A.; Tergau, F.; Paulus, W.; Priori, A. Safety criteria for transcranial direct current stimulation (tDCS) in humans [1] (multiple letters). Clin. Neurophysiol. 2003, 114, 2220-2223, doi:10.1016/S1388-2457(03)00235-9.

2. Poreisz, C.; Boros, K.; Antal, A.; Paulus, W. Safety aspects of transcranial direct current stimulation concerning healthy subjects and patients. Brain Res. Bull. 2007, 72, 208-214, doi:10.1016/j.brainresbull.2007.01.004.

3. Bikson, M.; Datta, A.; Elwassif, M. Establishing safety limits for transcranial direct current stimulation. Clin. Neurophysiol. 2009, 120, 1033-1034, doi:10.1016/j.clinph.2009.03.018.

4. Brunoni, A.R.; Amadera, J.; Berbel, B.; Volz, M.S.; Rizzerio, B.G.; Fregni, F. A systematic review on reporting and assessment of adverse effects associated with transcranial direct current stimulation. Int J Psychophysiol 2011, 14, 1133-45, doi:10.1017/S1461145710001690.

5. Aparício, L.V.M.; Guarienti, F.; Razza, L.B.; Carvalho, A.F.; Fregni, F.; Brunoni, A.R. A Systematic Review on the Acceptability and Tolerability of Transcranial Direct Current Stimulation Treatment in Neuropsychiatry Trials. Brain Stimul. 2016.

6. Zhao, H.; Qiao, L.; Fan, D.; Zhang, S.; Turel, O.; Li, Y.; Li, J.; Xue, G.; Chen, A.; He, Q. Modulation of brain activity with noninvasive transcranial direct current stimulation (tDCS): Clinical applications and safety concerns. Front. Psychol. 2017, 8.

7. Nitsche, M.A.; Bikson, M. Extending the parameter range for tDCS: Safety and tolerability of $4 \mathrm{~mA}$ stimulation. Brain Stimul. 2017, 10, 541-542, doi:10.1016/j.brs.2017.03.002. 
8. Bikson, M.; Grossman, P.; Thomas, C.; Zannou, A.L.; Jiang, J.; Adnan, T.; Mourdoukoutas, A.P.; Kronberg, G.; Truong, D.; Boggio, P.; et al. Safety of Transcranial Direct Current Stimulation: Evidence Based Update 2016. Brain Stimul. 2016, 9, 641661, doi:10.1016/j.brs.2016.06.004.

9. Buchanan, D.; D'Angiulli, A.; Samson, A.; Amare, S.; Gaumond, G.; Robaey, P. Making transcranial direct current stimulation treatment in atypical child and adolescent neurodevelopment a reality: Translating safety tolerability and acceptability evidence from the laboratory into the doctors office, the classroom, and home. Brain Stimul. 2019, 12, 474, doi:10.1016/j.brs.2018.12.545.

10. Gillick, B.T.; Feyma, T.; Menk, J.; Usset, M.; Vaith, A.; Wood, T.J.; Worthington, R.; Krach, L.E. Safety and feasibility of transcranial direct current stimulation in pediatric hemiparesis: randomized controlled preliminary study. Phys. Ther. 2015, 95, 337-49, doi:10.2522/ptj.20130565.

11. Zewdie, E.; Ciechanski, P.; Kuo, H.; Giuffre, A.; Kahl, C.; King, R.; Cole, L.; Grant, H.; Seeger, T.; Damji, O.; et al. Safety and tolerability of non-invasive neurostimulation in children. Brain Stimul. 2019, 12, 550, doi:10.1016/j.brs.2018.12.817.

12. Carlson, H.L.; Ciechanski, P.; Harris, A.D.; MacMaster, F.P.; Kirton, A. Changes in spectroscopic biomarkers after transcranial direct current stimulation in children with perinatal stroke. Brain Stimul. 2018, 11, 94-103, doi:10.1016/j.brs.2017.09.007.

13. Moliadze, V.; Andreas, S.; Lyzhko, E.; Schmanke, T.; Gurashvili, T.; Freitag, C.M.; Siniatchkin, M. Ten minutes of $1 \mathrm{~mA}$ transcranial direct current stimulation was well tolerated by children and adolescents: Self-reports and resting state EEG analysis. Brain Res. Bull. 2015, 119, 25-33, doi:10.1016/j.brainresbull.2015.09.011. 
14. Andrade, A.C.; Magnavita, G.M.; Allegro, J.V.B.N.; Neto, C.E.B.P.; Lucena, R. de C.S.; Fregni, F. Feasibility of Transcranial Direct Current Stimulation Use in Children Aged 5 to 12 Years. J. Child Neurol. 2013, 29, 0883073813503710 , doi:10.1177/0883073813503710.

15. Mattai, A.; Miller, R.; Weisinger, B.; Greenstein, D.; Bakalar, J.; Tossell, J.; David, C.; Wassermann, E.M.; Rapoport, J.; Gogtay, N. Tolerability of transcranial direct current stimulation in childhood-onset schizophrenia. Brain Stimul. 2011, 4, 275-280, doi:10.1016/j.brs.2011.01.001.

16. Gómez, L.; Vidal, B.; Maragoto, C.; Morales, L.M.; Berrillo, S.; Cuesta, H.V.; Baez, M.; Denis, M.; Marín, T.; Cabrera, Y.; et al. Non-invasive brain stimulation for children with autism spectrum disorders: A short-term outcome study. Behav. Sci. (Basel). 2017, 7, doi:10.3390/bs7030063.

17. Rich, T.L.; Nemanich, S.; Chen, M.; Friel, K.; Feyma, T.; Krach, L.; Nawshin, T.; Meekins, G.; Gillick, B.T. Clinical Study Transcranial Direct Current Stimulation (tDCS) Paired with Occupation-Centered Bimanual Training in Children with Unilateral Cerebral Palsy: A Preliminary Study. 2018, doi:10.1155/2018/9610812.

18. Nemanich, S.T.; Rich, T.L.; Chen, C.Y.; Menk, J.; Rudser, K.; Chen, M.; Meekins, G.; Gillick, B.T. Influence of combined transcranial direct current stimulation and motor training on corticospinal excitability in children with unilateral cerebral palsy. Front. Hum. Neurosci. 2019, 13, doi:10.3389/fnhum.2019.00137.

19. Zewdie, E.; Ciechanski, P.; Kuo, H.C.; Giuffre, A.; Kahl, C.; King, R.; Cole, L.; Godfrey, H.; Seeger, T.; Swansburg, R.; et al. Safety and tolerability of transcranial magnetic and direct current stimulation in children: Prospective single center evidence from 3.5 million 
stimulations. Brain Stimul. 2020, doi:10.1016/j.brs.2019.12.025.

20. Rouder, J.N.; Speckman, P.L.; Sun, D.; Morey, R.D.; Iverson, G. Bayesian t tests for accepting and rejecting the null hypothesis. Psychon. Bull. Rev. 2009, 16, 225-237.

21. Biel, A.L.; Friedrich, E.V.C. Why You Should Report Bayes Factors in Your Transcranial Brain Stimulation Studies. Front. Psychol. 2018, 9, 1125, doi:10.3389/fpsyg.2018.01125.

22. Dienes, Z.; Mclatchie, N. Four reasons to prefer Bayesian analyses over significance testing. Psychon. Bull. Rev. 2018, 25, 207-218, doi:10.3758/s13423-017-1266-z.

23. Jurcak, V.; Tsuzuki, D.; Dan, I. 10/20, 10/10, and 10/5 systems revisited: Their validity as relative head-surface-based positioning systems. Neuroimage 2007, doi:10.1016/j.neuroimage.2006.09.024.

24. Allen, J. Applying Study Results to Patient Care: Relative Risk, Absolute Risk, and Number Needed to Treat. Pharm. Lett. Prescr. 's Lett. 2005.

25. Citrome, L. Number needed to treat: What it is and what it isn't, and why every clinician should know how to calculate it. J. Clin. Psychiatry 2011.

26. Schachter, H.M.; Pham, B.; King, J.; Langford, S.; Moher, D. How efficacious and safe is short-acting methylphenidate for the treatment of attention-deficit disorder in children and adolescents? A meta-analysis. CMAJ 2001, 165, 1475-1488.

27. Buchanan, D.M.; D'Angiulli, A.; Samson, A.; Maisonneuve, A.R.; Robaey, P. Acceptability of transcranial direct current stimulation in children and adolescents with ADHD: The point of view of parents. J. Health Psychol. 2020, 135910532093705, doi:10.1177/1359105320937059. 


\section{Chapter 4:}

Acceptability of Transcranial Direct Current Stimulation in Children and Adolescents with ADHD: The Point of View of Parents 


\section{Study 3}

\section{ACCEPTABILITY OF TRANSCRANIAL DIRECT CURRENT STIMULATION IN CHILDREN AND ADOLESCENTS WITH ADHD: THE POINT OF VIEW OF PARENTS}

Buchanan, D.M.; D’Angiulli, A.; Samson, A.; Maisonneuve, A.R.; Robaey, P. Acceptability of transcranial direct current stimulation in children and adolescents with ADHD: The point of view of parents. J. Health Psychol. 2020, doi:10.1177/1359105320937059. This text is copied in full.

Authors: Buchanan DM ${ }^{\mathrm{a}, \mathrm{b}, \mathrm{c}}$, D'Angiulli A ${ }^{\mathrm{a}, \mathrm{b}}$, Samson A $\mathrm{e}^{\mathrm{e}}$, Maisonneuve $\mathrm{AR}^{\mathrm{f}}$, Robaey $\mathrm{P}^{\mathrm{a}, \mathrm{c}, \mathrm{d}}$

${ }^{a}$ Department of Neuroscience, Carleton University, Ottawa, ON, Canada;

${ }^{b}$ Neuroscience of Imagination Cognition Emotion Research Lab, Carleton University, Ottawa, ON, Canada;

${ }^{c}$ Neuropsychiatric Lab, Children's Hospital of Eastern Ontario, Ottawa, ON, Canada;

${ }^{d}$ Department of Psychiatry, University of Ottawa, Ottawa, ON, Canada;

${ }^{e}$ Faculty of Education, University of Ottawa, Ottawa, ON, Canada;

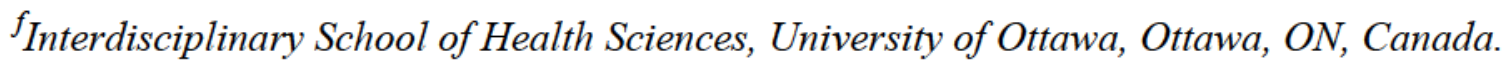

Corresponding Author: Derrick Matthew Buchanan, Neuroscience of Imagination Cognition Emotion Research Lab, Loeb B150, 1125 Colonel By Dr, K1S 5B6, Ottawa, ON, Canada. Email: matthewbuchanan@cmail.carleton.ca

Declarations of interest: none. 


\begin{abstract}
Transcranial direct current stimulation (tDCS) is a novel treatment option for attention deficit hyperactivity disorder (ADHD). To facilitate transferability, we investigated the perspectives of parents whose children have experienced tDCS using qualitative semi-structured interviews. A grounded theory approach using open, axial, and selective coding provided seven emergent themes: tDCS as hope for parents, safety tolerability and side effects of tDCS vs medication, burden of treatment, education and trust with care providers, cost and coverage, unestablished tDCS efficacy vs. established medication effectiveness, perceived compliance of tDCS vs medication. Results suggest tDCS acceptability depends on evidence of effectiveness and regular availability.
\end{abstract}

Key words: transcranial direct current stimulation; non-invasive brain stimulation; pediatric neuropsychiatry; attention deficit hyperactivity disorder; patient perspectives and acceptability 


\section{Introduction}

Attention deficit hyperactivity disorder (ADHD) is the most common neurodevelopmental condition and there remains to be any intensive long lasting treatment options. Current treatment methods are generally limited to long term commitments to pharmaceuticals, neurofeedback (NFB), and behavioural or psychosocial therapies which are not always effective or manageable for the parents and children. Transcranial direct current stimulation (tDCS) is a novel potential treatment option for children with ADHD (Breitling et al., 2016; Cosmo et al., 2015; Soff et al., 2017; Sotnikova et al., 2014) and other neuropsychiatric conditions (Lee et al., 2019; Rivera-Urbina et al., 2017) that may be more manageable than medication. tDCS is a non-invasive neuromodulation technique that utilizes low amplitude direct currents applied via scalp electrodes to modulate the level of excitability in specific brain regions (Giordano et al., 2017). While tDCS has mostly been used experimentally in the laboratory and in clinical trials it is in the process of becoming a standardized clinical tool (Giordano et al., 2017; Lefaucheur et al., 2017). There are several stages involved in this process such as establishing safety and tolerability, efficacy and effectiveness, and lastly transferability and dissemination (Steele et al., 2008). The present article will focus on the final stage of this process, transferability and dissemination.

Several reviews have been conducted to understand and compare the acceptability of pharmaceuticals with behavioural/psychosocial (Bennett et al., 1996; Johnston et al., 2008; Stuhec et al., 2015), with NFB (Catalá-López et al., 2017; Yan et al., 2019), and with other interventions for ADHD. In each of these studies the non-pharmacological approaches were found to be more acceptable despite often being less effective. In the studies by Demidovich (Demidovich et al., 2011) and Johnston (Johnston et al., 2010) it is also shown that acceptability 
can be a useful predictor for the likelihood of starting and continuing an ADHD treatment (Krain et al., 2005). Given the novelty of tDCS, studies evaluating its acceptability are scarce, and more so still in the context of ADHD. A major tDCS review from Bikson (Bikson et al., 2016) noted that tDCS is quite safe and tolerable in adults and so far appears to be in children; however only $5 \%$ of published research has been conducted in children. Each of these aforementioned studies are further limited through a narrow definition of acceptability, using only quantitative questionnaires or measuring rate of drop outs as a proxy. Studies rarely investigate the acceptability of tDCS beyond the narrowly operationalized 'rate of drop outs', let alone in pediatrics or ADHD. Although tDCS has proven to be very acceptable as defined by rate of drop outs (Bikson et al., 2016), there are several other nuances that must be considered to truly understand the acceptability of this specific intervention in a neuropsychiatric setting.

Sekhon, Cartwright, and Francis (Sekhon et al., 2017) meta-reviewed 43 reviews which measured acceptability of healthcare interventions. They found that 23 of these studies defined acceptability by rate of drop outs, where several other studies defined acceptability via cognition/perceptions, affect, or a combination of these. To better understand healthcare interventions, they developed the Theoretical Framework of Acceptability (TFA) which posits seven component constructs and the following definition: "Acceptability is a multi-faceted construct that reflects the extent to which people delivering or receiving a healthcare intervention consider it to be appropriate, based on anticipated or experienced cognitive and emotional responses to the intervention" (Sekhon et al., 2017: 8).

Acceptability focuses on one intervention, but preference requires an explicit comparison to another option. Preference between treatments options (between medications, or medication vs. psychosocial treatments) have been studies with parents. They consistently showed that 
treatment outcome is the most salient attribute for choice (Schatz et al., 2015). Preference for medication decreased for children of younger age and with milder symptoms (Waschbusch et al., 2011) but increased with time elapsed since diagnosis (DosReis et al., 2017). None of these studies considered tDCS, other brain stimulation or neurofeedback approaches.

\section{The Current Study}

The objective of this research is to gain a deeper understanding of families' experiences of tDCS use in their child and what makes it acceptable compared to medication. Given the subjective nature of each family's experience it is advantageous to utilize a qualitative inductive research approach (Glaser and Strauss, 1967; Taylor, 2013). Such an approach does not attempt to verify a specific construct or hypothesis per se, but instead aides in developing a deep understanding of the perceptions of participants through far-reaching analyses of their experiences. Using an inspired Grounded Theory (Taylor, 2013) approach we have obtained an integrative collection and analysis of parents' experiences and perceptions regarding tDCS use in their children.

Grounded Theory approaches the research process from an inductive position, with the ultimate goal of generating a theory based on themes that emerge from data collection: In this case, a theory of what makes tDCS acceptable. It allows the participants to describe their experiences in a profound way that is meaningful and comprehensive to them. It positions participants as the experts of their experiences allowing them to create their own narratives about tDCS. In this perspective, Grounded Theory will allow us to reach the specific objectives of this study. 


\section{Methods}

\section{Participant Sampling and Recruitment}

Participants from this acceptability study represent about half of the children from a larger sample of a separate study conducted by our group which investigated tDCS safety and tolerability in children and adolescents (Buchanan et al., 2019). Parents who enrolled one or more of their children in the safety study were subsequently asked if they would be willing to participate in our acceptability study. In the interest of emphasizing tDCS acceptability in children with ADHD, criterion based sampling was used (Lecompte and Goetz, 1982; Thé Riault and Gazzola, 2005). This included the youngest children with $\mathrm{ADHD}$; though the final sample did include a parent of two healthy controls.

\section{Participant Demographic Data}

Eight parents were recruited; representing a sample of 14 of their children ( 5 females) aged 6-14 (mean 9.21, SD 2.72). During the initial tDCS safety study parents and children participated in to two ten minute sessions of tDCS with randomized electrode locations and randomized amplitude (0-2mA). Quantitative safety and tolerability measures were taken and at least one parent was present throughout the entire study. The qualitative interviews were conducted on the parent who was present with their child during the initial safety study. Incidentally, the parental participants were all mothers. Joint Research Ethics was obtained from the Children's Hospital of Eastern Ontario (CHEO) and Carleton University. 


\section{Data Collection}

Qualitative semi-structured interviews (60-90 minutes) were conducted by the first author through a teleconferencing system enabled for digital recording. The recording was subsequently manually transcribed verbatim by a member of the research team. There was a total of 165 pages of text and 60698 words (12 pt Times New Roman, single space). Interviews were additionally evaluated using concurrent data analysis and collection, which allowed for initial interviews to inform subsequent themes. The researcher's interview questions and demographic questionnaire are available in the supplementary materials (Appendix C-1). Demographic data was collected but not reported given the omission of identifiers.

\section{Data Analysis}

Coding followed the grounded theory procedure (Taylor, 2013) and was completed twice by two separate researchers. The goal was to identify emerging themes utilizing: open, axial and selective coding. Data was first assessed using line-by-line open coding, in which significant units of text containing salient themes were labeled using descriptive codes. These are descriptive words or ideas which participants consistently used to convey their experiences and opinions. Next, these codes were synthesized into categories based on the units' common properties. The process of axial coding was then initiated, whereby the relationships between the categories were determined through the researcher's interpretive lens. Finally, selective coding of the data was engaged, whereby the categories and their relationships were interpreted to describe newly emerging themes regarding the use of tDCS in children and adolescents. Data analysis was completed using the NVivo qualitative software package (version 12). 


\section{Results}

Several themes emerged from the analysis of the interviews. These include: tDCS as a source of hope for parents, safety tolerability and side effects of tDCS vs medication, burden of treatment, education and trust with care providers, cost and coverage, unestablished tDCS efficacy vs. established medication effectiveness, perceived compliance of tDCS vs medication. The following sections describe these themes in detail and substantiate them through illustrative quotes from participants.

\section{tDCS as a Source of Hope for Parents}

Parents agreeing to enroll their children in a study regarding a potential novel medical treatment presumably do so in the hope that said treatment may at one point benefit their child. Herein parents have weighed the potential benefits of tDCS treatment against perceived cons. This theme was found in all transcripts:

"Certainly my motivation right away was yes I want to participate in this study because I just felt right off the bat that this is something that my child could benefit from.... I felt that it's important for us as a family, so my family and I, to do whatever we need to do right now to help advance any form of science that would help our child."

The perceived benefit to children at large also appeared to be equally important to parents as families hope for tDCS to become more accessible through regulatory agencies such as FDA and Health Canada: "It's my hope that it [tDCS] can be used in children and that we can see this happening in Canada sooner than later *laugh* and certainly I think without a doubt in adults, but I think certainly my hope is for children." Another parent notes the same: "Honestly I'm hoping we could get it actually passed [by a regulatory agency], so these kids could have this 
done rather than have to take the medication." One participant rationalized their hope: "I think the nice thing about the short and effective duration is that there is that hope of a light at the end of the tunnel." Another participant similarly noted, "I think there are a lot of parents who are hopeless right now that would jump on that idea."

In each of these cases the parents express their feelings and motivations to find a better treatment for their child and they hope that tDCS can be the answer. Participants further expressed that tDCS may also benefit and be accepted by other families with similar circumstances.

\section{Safety Tolerability and Side Effects of tDCS vs Medication}

It became clear that most parents experienced conflicting decisions regarding their child's use of medication, and were more acceptable towards their choice to pursue tDCS. For instance, one participant notes: "it's safer than having all these pills around because these days with kids, with everybody talking about overdosing and all that, then there would be no pills in the house, it would be safer." Another parent felt the same way: "Putting medication into your body which is equivalent to 'speed' is not something that parents like to do for their children; I mean these medications are addictive. If they are used wrongly on the streets they could be very dangerous so I can't imagine tDCS would have something worse than that."

From a more general point of view one parent pragmatically notes: "He's been on probably 4 or 5 different meds his whole life until we finally found the right one and certainly side effects of his meds are way worse." For another family, the medication actually does work well: "I already know that the medication helps... but I don't like the side effects and the fact that it's a Band-Aid and the days that he takes it he's good and the days he doesn't he's not." The 
same parent further expresses this through her discourse on not using medication at all: "He's

had some pretty serious side effects from medications and you know we've tried the whole route of no medication as well because most parents want to do that before they put the child on medication.”

On the other hand, participants appeared to feel quite good about tDCS safety and tolerability: "Based on all my children's experiences and my own I would rate [the safety] 10 out of $10 \ldots$ the procedure itself was very safe." In the same vein, another participant concurred by saying: "Well it seems pretty safe to me, like for me I would rate it a 10. I'd rather see him being on the stimulation [than on pharmaceuticals]." Finally, one parent summed up her feelings when she said "my children have had worse experiences at the dentist!"

Participants displayed a level of discomfort with various safety issues surrounding medication use and they see tDCS as a potential solution to this. Overall, it seems that the parent's preference to avoid side effects and maintain a lasting behavioural change motivates them toward tDCS despite the effectiveness of the medication.

\section{Burden of Treatment}

Participants viewed medication as a burden due to its daily and long term use, side effects, and the occasional hassle of filling prescriptions. All of this despite it being effective in most cases:

"He's actually responding very well to medication but ... you know, he's on six pills a day *laugh*... I'm still not quite there on the idea of fully embracing the fact that my child is going to be on medication his whole life, so yes if there was an alternative placed in front of me I would take the alternatives hands down." 
In contrast with medication, the potential perceived burden for tDCS was more concerned with the practicality of regularly attending the clinic. For example: "I mean it's definitely a lot especially for us because we live 25 to 30 minutes away... but I mean we do the things we have to do." From the point of view of participants, like medication, tDCS would also be more acceptable if it were available at home or remotely.

"My concern as a parent also would be, is this something that you always have to [travel] to in order to do it, or something that I can have this machine in my house and just switch it on for this time frame? Because that's another thing is the cost of transportation to get there, time off from work, or the convenience of it all."

Participants anticipate that the intense short duration of a tDCS treatment may be challenging but the same is expressed regarding years of medication use. Overall, parents perceive the accessibility of tDCS as a burden particularly compared to medication which is generally accessible at home and school.

\section{Education and Trust with Care Providers}

It was important for families comfort and acceptability of tDCS that they were educated on what to expect during the tDCS sessions.

"Once we sat down and we talked before we did the whole stimulation, you had explained everything perfect. The more information we had, it just cleared up the worry of what would have happened. I thought there was a lot more to be hooked up on his head. But seeing what was actually was hooked up was a big difference than what I had originally thought. I was like wow okay this is going to be easy."

It appears that the accurate provision of information attenuates potential concerns. Some participants sought to do this on their own as well: "So I looked at the pamphlet and read through and looked for information online which helped me with my decision to participate...I got from 
the pamphlet that it was definitely nothing like electroshock [therapy]." But some participants were put off by the name and idea of electrical stimulation:

"It doesn't sound inviting I can tell you that *laugh* like if you just went up to somebody and, like the words cranial and stimulation to me makes me think of wires in someone's brain, internally....It's a little bit of a daunting terminology but I mean most medical terms are anyway."

According to participants, another major determinant of whether or not to allow their child to use tDCS was: the trust and credibility of their healthcare providers, the organizing bodies associated with the study, and the researchers administering the intervention. In one case: "I was approached at my practitioner, my kid's doctor who has been seeing my kids for some time....Basically I put my trust in the organization which is a children's organization that I felt really comfortable that they will not put my kids in harm." Finally, trust with regards to the researchers administering the tDCS intervention was displayed by several participants: "I felt I trusted you and there was no way you would do this thing if you felt like it would hurt my kid."

Analyses demonstrate that for participants there is an absolute importance of establishing a rapport of trust with the provider. This trust is instilled by the reputation of the provider and the clear transmission of information to the participant.

\section{Cost and Coverage}

For participants, cost and coverage appears to be an important concern. "Cost always of course plays a factor. So if it was a covered cost and more effective, then it would be a no brainer to go and have [tDCS] done because it would be a lot less of the side effect." Another participant added, "Obviously money is a huge thing as well, I mean if I was looking at all my options and I had insurance coverage and everything was out on the table for me, then for sure [tDCS]." This 
participant also weighed the effectiveness with the cost: "Even if tDCS worked a little bit slower but didn't have all those nasty side effects of a pharmaceutical and even if it cost a little bit more, if I was able to do that then I would be inclined to do that." Finally, another expressed the same:

"It would have to be a pretty significant cost for me to say no, especially if it were more beneficial than the medication."

It appears that parents desire the benefits of tDCS treatment but that insurance / coverage of the treatment is important to their choice treatment modality and tDCS acceptability. The effectiveness however seems to play a more important role that could trump the burden of cost.

\section{Unestablished tDCS Efficacy vs. Established Medication Effectiveness}

The effectiveness of tDCS as a treatment in comparison to their child's current treatments was of particular interest to parents. "If there was proof of effectiveness that was comparable to medication, then that would be what would influence my decision [to choose tDCS over medication]." Interestingly parents for whom medication is ineffective and effective both share preference for tDCS if it is established as effective. In the case of a child who responds poorly to medication: "So we know with my child that pharmaceuticals have a lot of problems so if I could say that it was more effective to do the tDCS it would be no question I would do that immediately." Another participant elucidates this, indicating that even if the tDCS intervention proved to be somewhat less effective than pharmaceuticals, they would still opt for the non-drug route: "If you compare to medication for instance, if I put the medication at $100 \%$ effective for example and I put the tDCS at 50\% maybe I'll say it's not good enough. But if it's at $75 \%$ or $80 \%$ as good as the medication with much less side effects then I'll stay there." 
There is a perceived consensus among participants that if tDCS effectiveness is demonstrated that they would opt for the stimulation intervention over pharmaceutical approaches.

\section{Perceived compliance of tDCS vs Medication}

The duration and frequency of intervention "doses" and the maintenance of the treatment present some facets associated with the perceived compliance. Participants displayed a willingness and hope toward an intensive short term treatment option: "I would be more willing to put the time and energy into very intensive but shorter duration therapy versus the long haul [years of medication]." Another participant similarly notes "If you're able to do something for a

short span of time and see results as opposed to medication where you're always switching doses and it's going to be a lifelong thing, that's a big factor in making the decision about which method we're going to use."

On the other hand, many participants indicated that the time it would take to see a positive change in their child's behaviour would also affect their acceptance: "Another factor is maybe how quickly you're going to see results... Well if you're doing this for 6 months then you might see a result, versus some people might say well I've been giving him medication for 2-3 weeks and I'm already going to start to see results." Finally, one parent also considered the idea of accepting a combination of medication and tDCS. "You might be able to... use [tDCS] in combination with a lower dose of the medication to get the same or improved effectiveness."

Participants generally expressed their compliance toward a short term intensive tDCS treatment compared to years of medication. That being said, the unspecific relief onset and 
currently unestalblished efficacy of tDCS is an important concern for parents as they consider tDCS as a treatment.

In summary, the analysis showed that the there are many components contributing to parents perceptions of the acceptability of tDCS. Acceptability of a new treatment is always relative to existing treatment. In general, families perceived tDCS as being acceptable when compared to medications. Parents hope for an alternative to medication that would be personally and socially more acceptable. The themes identified in the interviews seem to define the conditions for a new approach like tDCS to be acceptable: the new treatment should demonstrate efficacy and safety; be practical in terms of time, cost, coverage and availability; knowledge transfer to users is important to understand procedures and define expectation, and trust and reputation are imperative. Finally, the branding and terminology may be a barrier for adoption.

\section{Discussion}

Understanding the acceptability of a healthcare intervention is an imperative step in the process of transferability and dissemination from research to practice (Steele et al., 2008). At the 2020 NYC Neuromodulation Conference (https://youtu.be/PhzyPSZrZSo) several speakers explicitly discussed the unanswered community demand for tDCS and the onus of researchers to transfer evidence based tDCS protocols into community medicine. In the case of children with ADHD one imperative step is to understand the parents' perspectives on tDCS acceptability. Until now, perceptions of parents of children exposed to tDCS have not been investigated. The present study put the focus on the child and the parent. This led to several novel findings important for the translation of tDCS transferability and future translational research. One finding that permeated throughout each interview was hope. In the healthcare literature, hope has 
often been viewed as an essential perquisite to coping and adaptation to illness (Samson et al., 2009).

Hope provides the overarching recurring drive which connects the whole of participant's decision making. In the case of tDCS, this appears to begin with hope for a cure, or at least a more manageable short term intensive treatment option than medication. Actually enrolling their child in the study seemed to be a further extension of this as a hope to advance science. After experiencing tDCS first hand in the original trial, seeing the lack of side effects and learning about how it works, this manifested a new more specific hope. Hope that tDCS efficacy will be established and that it will become regulated through government health institutions. Ultimately, this translates to hope that tDCS can help their child and that it will provide better results than their previous options. This hope is the mirror image of the frustrations with medical treatment. Despite the medication, the children continue to struggle with symptoms, especially inattention, academic and social difficulties. The burden on families remains considerable. Finally, if medication reduces symptoms and impacts, concerns about side effects and being overly subdued remain among parents and adolescents (Sikirica et al., 2015). This hope is also the reflection of the desire to maximize symptom control and outcome found in preference studies (Nafees et al., 2014; Schatz et al., 2015).

Overall, parents find tDCS to be an acceptable treatment option. While this is encouraging from the point of view of parents, it is possible that the novelty of the treatment may reinvigorate their outlook toward their child's prognosis. It is the responsibility of the medical and scientific community to not engender unrealistic expectations surrounding a novel treatment. 
Perhaps one of the most important incidental findings is that acceptability, much like consent, is an ongoing process. It begins with the participant's willingness to participate in the study, it becomes concurrent during the actual physical trial itself, and it becomes prospective in the context of continuing a trial or pursuing the treatment after the trial has ended. This is important because it highlights how tDCS acceptability is part and parcel to the ongoing decision making process of the participants.

After analyzing the data through the lens of Grounded Theory it became clear that many of the themes which emerged also closely coincided with the Theoretical Framework of Acceptability (Sekhon et al., 2017). The TFA describes seven criteria for understanding the acceptability of a new healthcare intervention: affective attitude, burden, ethicality, intervention coherence, opportunity costs, perceived effectiveness, and self-efficacy. Aligned with our results, the TFA also demonstrates that acceptability is an ongoing process (Appendix C-2).

Parents specifically expressed an interest in dosage of tDCS and the idea of combining or lowering medication dose by using tDCS as an adjunct. Notably, recent tDCS titration research from Samani, Agboada, Jamil, Kuo, and Nitsche (Samani et al., 2019) have begun to address these types of questions. Parents also hoped that tDCS would become properly regulated for neuropsychiatric care, primarily for insurance, accessibility, and safety reasons. To that end the recent tDCS Neuropsychiatric guidelines from Vicario \& Nitsche (Vicario and Nitsche, 2019) are great steps forward. Lastly, parents were concerned that the accessibility of tDCS may be a challenge and some brought up the idea of using the device at home. Indeed, recent studies have found good compliance and outcomes using an at home tDCS approach (André et al., 2016; Charvet et al., 2018; Riggs et al., 2018). In addition, Knotkova and colleagues (Knotkova et al., 2019) have published a practical guideline for at home use. 
Some limitations of the present study that are worth noting include the implicit selection bias. Since our trial only interviewed families who participated in our RCT they presumably were already comfortable with tDCS to some degree. It would have also been advantageous to interview families who declined to participate in the RCT as their opinions may be less supportive of tDCS. Indeed, we had originally intended to do this, however the sample size was insufficient and practically speaking it would not be possible to only recruit participants who declined participation. Moreover, the notion that tDCS had some apriori acceptability was confirmed by our analysis and is in line with the theoretical framework of acceptability (Appendix C-2). Another implicit limitation that may influence tDCS acceptability is its efficacy. Since the efficacy of tDCS is not yet established and one component of acceptability is based on efficacy the interpretation of tDCS acceptability in the present sample is limited. This was confirmed in our grounded theory analysis as establishing efficacy emerged as one of the salient themes.

\section{Conclusion}

Participants all had a direct experience of two tDCS sessions. With this experience, combined with the parents' trust in the hospital conducting the research, and the lack of side effects, they were consistently partial to tDCS vs medication; viewing it as more manageable and revealing their hope toward the option of an intensive short term treatment for ADHD. This implies that families who trust their healthcare provider and are educated about tDCS would be more willing to try it as a treatment if it were prescribed. Participants' acceptance of tDCS, however, came with a general contingency of establishing comparable efficacy to medication. Our conclusions are mostly limited to the primary school age range. Further research 
investigating tDCS acceptability may focus on younger preschool children or adolescents, age group where medication avoidance and refusal are more frequent, respectively.

\section{Practice Implications}

This study provides insight regarding parents' point of view of tDCS use in their child. Clinicians and researchers that plan to utilize tDCS, particularly in children, should consider this article as a guideline to relate to parents and to implement a protocol that is considerate of their needs, their motivations, and their perceptions. As presented here, the parents' points of view are imperative during phases of transferability. Overall, it appears that parents are hopeful to pursue tDCS as a treatment option in their child once its efficacy is established and it becomes regularly available. This should alleviate apprehension surrounding tDCS use in children and encourage future research and clinical use. 


\section{References}

André S, Heinrich S, Kayser F, et al. (2016) At-home tDCS of the left dorsolateral prefrontal cortex improves visual short-term memory in mild vascular dementia. Journal of the Neurological Sciences. DOI: 10.1016/j.jns.2016.07.065.

Bennett DS, Power TJ, Rostain AL, et al. (1996) Parent acceptability and feasibility of ADHD interventions: Assessment, correlates, and predictive validity. Journal of Pediatric Psychology. DOI: 10.1093/jpepsy/21.5.643.

Bikson M, Grossman P, Thomas C, et al. (2016) Safety of Transcranial Direct Current Stimulation: Evidence Based Update 2016. Brain Stimulation 9(5): 641-661. DOI: 10.1016/j.brs.2016.06.004.

Breitling C, Zaehle T, Dannhauer M, et al. (2016) Improving Interference Control in ADHD Patients with Transcranial Direct Current Stimulation (tDCS). Frontiers in Cellular Neuroscience 10. DOI: 10.3389/fncel.2016.00072.

Buchanan D, D'Angiulli A, Samson A, et al. (2019) Making transcranial direct current stimulation treatment in atypical child and adolescent neurodevelopment a reality: Translating safety tolerability and acceptability evidence from the laboratory into the doctors office, the classroom, and home. Brain Stimulation 12(2). Elsevier: 474. DOI: 10.1016/j.brs.2018.12.545.

Catalá-López F, Hutton B, Núñez-Beltrán A, et al. (2017) The pharmacological and nonpharmacological treatment of attention deficit hyperactivity disorder in children and adolescents: A systematic review with network meta-analyses of randomised trials. PLoS ONE. DOI: 10.1371/journal.pone.0180355.

Charvet L, Shaw M, Dobbs B, et al. (2018) Remotely Supervised Transcranial Direct Current 
Stimulation Increases the Benefit of At-Home Cognitive Training in Multiple Sclerosis. Neuromodulation. DOI: 10.1111/ner.12583.

Cosmo C, Baptista AF, De Araújo AN, et al. (2015) A randomized, double-blind, shamcontrolled trial of transcranial direct current stimulation in attention-deficit/hyperactivity disorder. PLoS ONE 10(8). DOI: 10.1371/journal.pone.0135371.

Demidovich M, Kolko DJ, Bukstein OG, et al. (2011) Medication Refusal in Children with Oppositional Defiant Disorder or Conduct Disorder and Comorbid AttentionDeficit/Hyperactivity Disorder: Medication History and Clinical Correlates. Journal of Child and Adolescent Psychopharmacology. DOI: 10.1089/cap.2010.0001.

DosReis S, Park A, Ng X, et al. (2017) Caregiver treatment preferences for children with a new versus existing attention-deficit/hyperactivity disorder diagnosis. Journal of Child and Adolescent Psychopharmacology 27(3). Mary Ann Liebert Inc.: 234-242. DOI: 10.1089/cap.2016.0157.

Giordano J, Bikson M, Kappenman ES, et al. (2017) Mechanisms and Effects of Transcranial Direct Current Stimulation. Dose-response : a publication of International Hormesis Society 15(1). SAGE Publications: 1559325816685467. DOI: 10.1177/1559325816685467. Glaser B and Strauss A (1967) Grounded Theory: The Discovery of Grounded Theory. Sociology The Journal Of The British Sociological Association.

Johnston C, Hommersen P and Seipp C (2008) Acceptability of Behavioral and Pharmacological Treatments for Attention-Deficit/Hyperactivity Disorder: Relations to Child and Parent Characteristics. Behavior Therapy. DOI: 10.1016/j.beth.2007.04.002.

Johnston C, Mah JWT and Regambal M (2010) Parenting Cognitions and Treatment Beliefs as Predictors of Experience Using Behavioral Parenting Strategies in Families of Children 
With Attention-Deficit/Hyperactivity Disorder. Behavior Therapy. DOI:

10.1016/j.beth.2010.02.001.

Knotkova H, Clayton A, Stevens M, et al. (2019) Home-Based Patient-Delivered Remotely Supervised Transcranial Direct Current Stimulation. In: Practical Guide to Transcranial Direct Current Stimulation. Cham: Springer International Publishing, pp. 379-405. DOI: 10.1007/978-3-319-95948-1 13.

Krain AL, Kendall PC and Power TJ (2005) The role of treatment acceptability in the initiation of treatment for ADHD. Journal of Attention Disorders. DOI: 10.1177/1087054705279996.

Lecompte M d. and Goetz JP (1982) Ethnographic Data Collection in Evaluation Research. Educational Evaluation and Policy Analysis. DOI: 10.3102/01623737004003387.

Lee JC, Kenney-Jung DL, Blacker CJ, et al. (2019) Transcranial Direct Current Stimulation in Child and Adolescent Psychiatric Disorders. Child and Adolescent Psychiatric Clinics of North America. DOI: 10.1016/j.chc.2018.07.009.

Lefaucheur J-P, Antal A, Ayache SS, et al. (2017) Evidence-based guidelines on the therapeutic use of transcranial direct current stimulation (tDCS). Clinical Neurophysiology 128(1): 5692. DOI: 10.1016/j.clinph.2016.10.087.

Nafees B, Setyawan J, Lloyd A, et al. (2014) Parent preferences regarding stimulant therapies for ADHD: a comparison across six European countries. European Child and Adolescent Psychiatry 23(12). Dr. Dietrich Steinkopff Verlag GmbH and Co. KG: 1189-1200. DOI: 10.1007/s00787-013-0515-6.

Riggs A, Patel V, Paneri B, et al. (2018) At-Home Transcranial Direct Current Stimulation (tDCS) With Telehealth Support for Symptom Control in Chronically-Ill Patients With Multiple Symptoms. Frontiers in Behavioral Neuroscience. DOI: 
10.3389/fnbeh.2018.00093.

Rivera-Urbina GN, Nitsche MA, Vicario CM, et al. (2017) Applications of transcranial direct current stimulation in children and pediatrics. Reviews in the Neurosciences 28(2): 173184. DOI: 10.1515/revneuro-2016-0045.

Samani MM, Agboada D, Jamil A, et al. (2019) Titrating the neuroplastic effects of cathodal transcranial direct current stimulation (tDCS) over the primary motor cortex. Cortex. Elsevier. DOI: 10.1016/J.CORTEX.2019.04.016.

Samson A, Tomiak E, Dimillo J, et al. (2009) The lived experience of hope among parents of a child with Duchenne muscular dystrophy: Perceiving the human being beyond the illness. Chronic Illness. DOI: 10.1177/1742395309104343.

Schatz NK, Fabiano GA, Cunningham CE, et al. (2015) Systematic Review of Patients' and Parents' Preferences for ADHD Treatment Options and Processes of Care. The patient 8(6). Springer International Publishing: 483-97. DOI: 10.1007/s40271-015-0112-5.

Sekhon M, Cartwright M and Francis JJ (2017) Acceptability of healthcare interventions: An overview of reviews and development of a theoretical framework. BMC Health Services Research. DOI: 10.1186/s12913-017-2031-8.

Sikirica V, Flood E, Dietrich CN, et al. (2015) Unmet Needs Associated with AttentionDeficit/Hyperactivity Disorder in Eight European Countries as Reported by Caregivers and Adolescents: Results from Qualitative Research. Patient 8(3). Springer International Publishing: 269-281. DOI: 10.1007/s40271-014-0083-y.

Soff C, Sotnikova A, Christiansen H, et al. (2017) Transcranial direct current stimulation improves clinical symptoms in adolescents with attention deficit hyperactivity disorder. Journal of Neural Transmission 124(1). DOI: 10.1007/s00702-016-1646-y. 
Sotnikova A, Soff C, Becker K, et al. (2014) Transcranial direct current stimulation (tDCS) in the treatment of adolescents with attention deficit hyperactivity disorder (ADHD). Clinical neurophysiology 125: S225-s226. DOI: 10.1007/s00702-016-1646-y.

Steele RG, Elkin TD and Roberts MC (eds) (2008) Handbook of Evidence-Based Therapies for Children and Adolescents. Issues in Clinical Child Psychology. The British Journal of Psychiatry. Springer. DOI: 10.1192/bjp.112.483.211-a.

Stuhec M, Munda B, Svab V, et al. (2015) Comparative efficacy and acceptability of atomoxetine, lisdexamfetamine, bupropion and methylphenidate in treatment of attention deficit hyperactivity disorder in children and adolescents: A meta-analysis with focus on bupropion. Journal of Affective Disorders. DOI: 10.1016/j.jad.2015.03.006.

Taylor B (2013) Grounded theory. In: Qualitative Research in the Health Sciences: Methodologies, Methods and Processes. DOI: 10.4324/9780203777176.

Thé Riault A and Gazzola N (n.d.) Feelings of inadequacy, insecurity, and incompetence among experienced therapists. DOI: 10.1080/14733140512331343840.

Vicario CM and Nitsche MA (2019) tDCS in Pediatric Neuropsychiatric Disorders. Neurotechnology and Brain Stimulation in Pediatric Psychiatric and Neurodevelopmental Disorders. Academic Press: 217-235. DOI: 10.1016/B978-0-12-812777-3.00009-X.

Waschbusch DA, Cunningham CE, Pelham WE, et al. (2011) A discrete choice conjoint experiment to evaluate parent preferences for treatment of young, medication naïve children with ADHD. Journal of Clinical Child and Adolescent Psychology 40(4). Routledge: 546561. DOI: $10.1080 / 15374416.2011 .581617$.

Yan L, Wang S, Yuan Y, et al. (2019) Effects of neurofeedback versus methylphenidate for the treatment of ADHD: Systematic review and meta-analysis of head-to-head trials. Evidence- 
Based Mental Health. DOI: 10.1136/ebmental-2019-300088. 


\section{Chapter 5:}

High and Low Clusters of Theta Beta Ratio have no Relationship with Attention Deficit Hyperactivity Disorder 


\section{Study 4}

\section{HIGH AND LOW CLUSTERS OF THETA BETA RATIO HAVE NO RELATIONSHIP WITH}

ATTENTION DEFICIT HYPERACTIVITY DISORDER

Buchanan, D.M.; D'Angiulli, A.; Robaey, P. High and Low Clusters of Theta Beta Ratio have no Relationship with Attention Deficit Hyperactivity Disorder. In preparation for submission to Biological Psychiatry.

Authors: Buchanan $\mathrm{DM}^{\mathrm{a}, \mathrm{b}, \mathrm{c}}, \mathrm{D}^{\prime}$ Angiulli $\mathrm{A}^{\mathrm{a}, \mathrm{b}}$, Robaey $\mathrm{P}^{\mathrm{a}, \mathrm{c}, d}$

${ }^{a}$ Department of Neuroscience, Carleton University, Ottawa, ON, Canada;

${ }^{b}$ Neuroscience of Imagery Cognition Emotion Research Lab, Carleton University, Ottawa, ON, Canada;

${ }^{c}$ Neuropsychiatric Lab, Children's Hospital of Eastern Ontario, Ottawa, ON, Canada;

${ }^{d}$ Department of Psychiatry, University of Ottawa, Ottawa, ON, Canada.

Corresponding Author: Derrick Matthew Buchanan, Neuroscience of Imagination Cognition Emotion Research Lab, Loeb B150, 1125 Colonel By Dr, K1S 5B6, Ottawa, ON, Canada. Email: matthewbuchanan@cmail.carleton.ca

Declarations of interest: none. 
Abstract

Background: Attention deficit hyperactivity disorder (ADHD) is the most common neurodevelopmental condition worldwide. Perhaps the most highly cited and debated biological marker of $\mathrm{ADHD}$ is the theta/beta ratio (TBR). The TBR is an electrophysiological biomarker derived from electroencephalography (EEG) recordings. TBR has been proposed to enhance diagnostics/prognostics and to act as a treatment target in ADHD.

Objective: To test the validity of TBR and the idea of a TBR threshold in relation to ADHD. Methods: The present study aimed to replicate the EEG TBR clustering analysis of Bussalb et al [1], in a normative age controlled sample (all subjects 8 years old) $(n=189)$. Our clusters were validated using machine learning support vector analysis. We analyzed high and low TBR clusters, and sex, in the context of ADHD symptomology.

Results: In this study we found strong evidence for high and low clusters of TBR. These clusters yielded a TBR threshold in healthy controls that is strikingly similar to what Bussalb et al [1] reported in an ADHD sample. $34 \%$ of our healthy sample, compared to $36 \%$ of Bussalb's ADHD sample fell above the same threshold. We also demonstrated that these clusters could not be differentiated by ADHD symptomology, or sex, but that sex could be partially differentiated by ADHD symptomology.

Conclusion: This study provided evidence that there is no relationship between TBR distributions in ADHD and its subtypes. Therefore, regardless of clustering, TBR does not appear to be a valid biomarker in ADHD.

Keywords: ADHD, theta/beta ratio, TBR, EEG, clustering, inattentive, hyperactive, arousal 


\section{Introduction}

Attention deficit hyperactivity disorder (ADHD) is the most common neurodevelopmental condition affecting up to $5 \%$ of children and adolescents around the world $[2,3]$. Perhaps the most highly cited and debated biological marker of ADHD is the theta/beta ratio (TBR) [4]. The TBR is an electrophysiological biomarker derived from electroencephalography (EEG) recordings and computed by dividing theta power (microvoltage measured between $4-8 \mathrm{~Hz}$ ) by beta power (microvoltage measured between $12-30 \mathrm{~Hz}$ ). The clinical importance of TBR, like any biomarker, is to enhance diagnostics/prognostics and to act as a target in treatment models. At the moment, the efficacy of TBR in both the former and latter capacities remains highly controversial [5].

\section{Diagnostics and Prognostics using TBR}

Some authors have suggested that theta (associated with drowsiness/inattention) appears to be elevated compared to beta (associated with awake/attentive) in ADHD patients versus healthy controls (HC) [6-8] and that TBR values could be used to help diagnose ADHD $[9,10]$. An early meta-analysis from Snyder [7] $(\mathrm{n}=1498)$ reported an effect size of 3.08 for TBR recorded at $\mathrm{Cz}$ (sensory motor area) for differentiating $\mathrm{ADHD}$ from healthy controls. This eventually lead to TBR at $\mathrm{Cz}$ being approved by the FDA as a diagnostic aid for ADHD [11] in 2013.

However, the literature appears to have taken a step back since then. A number of more recent studies [12-14] have questioned the FDA approval after finding no TBR differences between healthy controls and children with ADHD. A meta-analyses from Loo et al [15] in 871 participants (590 youth, 276 adults) demonstrated no difference in TBR at $\mathrm{Cz}$ in youth with 
ADHD vs healthy age and sex matched children. Another meta-analyses from Arns et al [8] in 1253 children with ADHD and 517 healthy controls concluded that increased TBR at Cz cannot be considered a reliable diagnostic marker of ADHD but rather may offer some prognostic value only. Therefore, according to most recent evidence, TBR does not appear to have meaningful clinical diagnostic value in children with ADHD.

\section{TBR as a Treatment Target}

The efficacy of TBR at $\mathrm{Cz}$ has also been investigated as a biological index/target for clinical treatment in ADHD using neurofeedback (NFB) [16] or methylphenidate [17]. Neurofeedback training utilizes a form of operant conditioning which trains children to modulate their endogenous brain activity based on real time feedback of EEG indices. Methylphenidate on the other hand works by inhibiting the reuptake of norepinephrine (NE) and dopamine (DA) by modulating the activity of NE and DA transporters. If TBR is indeed an index of ADHD, modulating it with neurofeedback or methylphenidate should be associated with improvement in ADHD symptoms.

Indeed, this hypothesis has been tested for neurofeedback by Arnold et al [18] in a double-blind sham controlled trial $(n=39)$ in children with ADHD. This study aimed to reduce theta amplitude and increase beta amplitude (ie., decrease the TBR) over 40 sessions of NFB (2$3 \mathrm{x}$ weekly). Both active and sham conditions led to reductions in pre-post parent ratings of ADHD, but active NFB was not superior to sham. In fact, sham was actually nominally better than active NFB. The authors unfortunately did not report if TBR values changed pre-post intervention. This would have indicated if the NFB training was successful in the first place, and whether or not it could have explained some of the improvement in ADHD symptoms. The 
largest adult sample ( $\mathrm{n}=118)$ to date was recently published and it precisely corroborates Arnold's [18] results, and extends them by measuring TBR pre-post intervention [16]. Shonenberg et al [16] conducted a triple-blind randomized controlled neurofeedback trial using TBR as a target (at $\mathrm{Cz}, \mathrm{Fz}, \mathrm{Pz}$ ) 2x a week for 15 weeks. Similar to Arnold et al [18], Shonenberg [16] found that both conditions produced a significant reduction in ADHD symptoms, but active modulation of TBR was not superior to sham neurofeedback. Moreover, when they evaluated the pre-post TBR they found virtually no change in TBR, nor did they find any difference in TBR changes between active NFB vs sham NFB. This seems to suggest that TBR is not an index of pathology.

Overall, this means that the significant reduction in ADHD cannot have had anything to do with TBR. This is further evidenced by a sample of 336 medication naïve (or washed out) children with ADHD and 158 healthy controls [17]. All of the children had EEG assessments to measure TBR (at Fz, FCz, Cz) before and after 4-6 weeks of methylphenidate (in ADHD children). The authors did find significant improvement in ADHD, but they did not find any difference between TBR in children with ADHD and healthy controls at the baseline, nor did they find any difference in TBR pre-post methylphenidate intervention. TBR was also not able to predict treatment response. Therefore TBR does not appear to be a clinically relevant biomarker in neuromodulatory or methylphenidate treatment trials.

\section{Can TBR be Saved?}

In an attempt to reconcile some of the negative clinical results surrounding TBR, some studies have suggested that treatment samples need to be selective and only include patients with excessively high TBR $[1,18,19]$. Their hypothesis is that this may lead to better response curves, 
and that patients with low TBR are otherwise obscuring the data in these large clinical trials. To that end, Bussalb et al [1] aimed to validate the idea of a TBR threshold such that it could be used as an inclusion criteria for clinical trials. They did this by clustering TBR values from 363 patients with ADHD (derived from three separate datasets) into two clusters. Their analysis yielded two overlapping high $(\mathrm{n}=131, \mu=4.8)$ and low $(\mathrm{n}=232, \mu=2.66)$ TBR clusters with an averaged threshold of 3.706. Bussalb stated that the significance of these clusters was to validate a TBR threshold for clinical trials in ADHD, but they never actually reported if ADHD severity bore any relationship on the high and low TBR clusters. In other words, the authors identified two distributions but did not investigate if either distribution could be explained by ADHD symptomology or severity. Thus, there is no actual pathological implication of TBR in Bussalb's sample. In fact, the only biological factor that explained significant variation in Bussalb's high and low clusters was age. Given that TBR is known to decrease with aging $[9,13,20]$ the TBR clusters found by Bussalb et al [1] may not have anything at all to do with ADHD. If high and low TBR clusters exist but have no relationship with ADHD then the "so called" TBR threshold is clinically irrelevant.

\section{The Current Study}

To test our hypotheses, the present study aimed to replicate the clustering analysis of Bussalb et al in a normative, age controlled, sample (all subjects 8 years old) $(\mathrm{n}=189)$. Although these children were mostly typically developing they all underwent a semi-structured psychological evaluation for ADHD and completed an EEG assessment. The semi-structured interviews allowed us to evaluate children who did not necessarily meet the criteria for ADHD but still had inattentive or hyperactive problems. We hypothesized that there would be two distinguishable high and low clusters of TBR in neurotypical children and that these clusters 
would have no relationship with ADHD. The implication is that high and low TBR clusters, and TBR in general, may not actually be a useful index for ADHD diagnostics, prognostics, or treatment models. Given that there are multiple clinical trials [21,22] (NCT02251743, NCT02778360) and likely new research proposals being funded on the principle of TBR thresholds the results of this study may disrupt the literature.

\section{Methods}

\section{Participants}

The current study consisted of mostly typically developing 8 year old children from three different locations (Ste-Justine, Notre-Dame, and À Domicile) in Montreal, Canada between 2004 and 2005. Participants are part of the ongoing Quebec New Born Twin Study. Our cohort consisted of all subjects who received a complete psychological assessment and a complete and useable electroencephalography recording $(n=189)$. The present sample consisted of 75 monozygotic twins (35 males, 40 females) and 114 dizygotic twins (61 males, 53 females). Zygosity was determined by using DNA sampling and questions relating to physical similarity. That being said, zygosity bares no influence on our current hypothesis and therefore for the purposes of our analysis all subjects were treated as individuals. For descriptive purposes, boxplots for zygosity in relation to cluster and ADHD are available in Appendix D-1. Written and informed consent was obtained from all parents.

\section{Data Collection}

\section{Psychological Assessment}

All of the subjects underwent semi-structured interviews where parents scored their 
children's strengths and weaknesses in relation to ADHD symptoms. The diagnosis/evaluation of ADHD was obtained using criteria from the DSM-IV-TR [23]. According to the DSM-IV-TR, the impairments related to $\mathrm{ADHD}$ are classified and measured into two types of symptomologies: inattention and hyperactivity/impulsivity. The inattentive subtype may be described by a child's inability to regulate or maintain attention when focusing on a specific task whereas the hyperactive/impulsive subtype is characterized by inappropriate responses or an inability to inhibit a response to a specific situation without careful consideration. Under this definition there are 9 symptoms associated with the inattentive subtype and 9 associated with the hyperactive/impulsive subtype (Table 1).

Table 1. Symptoms of ADHD categorized by two different symptomologies: Inattention and Hyperactivity/Impulsivity

1. Makes careless mistakes.

1. Fidgets

2. Difficulty maintaining attention.

2. Difficulty remaining in seat.

3. Does not listen when spoken directly.

3. Often runs or climbs excessively.

4. Does not complete instructions.

4. Difficulty playing quietly.

5. Poor self-organization.

5. Excessive motor activity.

6. Avoid mental effort tasks.

6. Talks excessively.

7. Loses things.

7. Blurts out answers.

8. Easily distracted.

8. Difficulty waiting for turn.

9. Forgetful.

9. Often interrupts others.

The clinical assessment of ADHD has five conditions in order for a child to be appropriately diagnosed. First, a child must present at least six to nine inattentive and/or hyperactivity/impulsivity symptoms. Second, symptoms of inattention and/or hyperactivity/impulsivity must be present before the age of seven. Third, the symptoms must be ongoing for at least six months. Fourth, symptoms of impairment must occur in at least two 
different settings (ie., school, occupational, and/or home environments). Fifth and lastly, the symptoms of impairment cannot be exclusive to or be better explained by another psychiatric disorder.

Depending on the criteria met from the two symptomologies, it is possible for a child to be diagnosed to three different subtypes: predominately inattentive type (ADHD-I), predominately hyperactivity/impulsivity type (ADHD-H), and combined type (ADHD-C). The qualification for ADHD-I requires at least six inattentive symptoms, ADHD-H requires at least six hyperactivity/impulsivity symptoms, and ADHD-C requires at least six of both symptoms (Table 2).

Table 2. ADHD Diagnostic Criteria by DSM-IV-TR

Subtype Criteria

ADHD-I

- Six or more inattentive symptoms needed to qualify for ADHD-I.

ADHD-H

- Six or more hyperactivity/impulsivity symptoms needed to qualify for ADHD-H.

ADHD-C

- Six or more from both inattention and hyperactivity/impulsivity symptoms needed to qualify for ADHD-C.
Other Diagnostic Criteria

a. Hyperactivity/impulsivity and inattention symptoms must cause impairment before age seven.

b. Symptoms must be present for at least six months.

c. The impairment of symptoms must be present in at least two settings (school, work, home).

d. Symptoms are not exclusively in other disorders such as schizophrenia, pervasive developmental disorder, or other psychiatric disorder, or cannot be better explained by another condition.

All 189 subjects were evaluated for these 18 symptoms. Furthermore, a continuous scale was also applied to each symptom in order to understand the strengths and weaknesses of each subject. This was based on a 7-point system ( 3 = extreme weakness; 2 = significant weakness; -1 
$=$ normal $; 0=$ normal; $1=$ normal; $2=$ significant strength; $3=$ extreme strength). By combining the 7-point behavioural scoring system with the EEG measures outlined in the next section, it was possible to evaluate the full variability of the so called EEG TBR phenotype in relation to ADHD.

\section{EEG Assessment}

In addition to the psychological assessment, all 189 subjects underwent 3 minutes of resting-state eyes open 56-channel electroencephalography (EEG) in an acoustically and electronically shielded room. Signals were recorded using InstEP EEG acquisition software, and an ElectroCap designed according to the international 10-20 system, connected to a Sensorium System (model EPA-6) amplifier. Assessments were performed with subjects seated in a dimly lit room with an EEG technician present. Verbal instructions included sitting upright, remaining still, avoiding jaw clenching and blinking, fixating gaze, and remaining awake. Electrogel was applied to each electrode via syringe with EEG impedance maintained below $5 \mathrm{k} \Omega$, and electromyography (EMG) impedance maintained below $25 \mathrm{k} \Omega$. Electrooculography (EOG) channels were also recorded from during EEG. For the purpose of this study, EMG and EOG were only used to detect and remove artifacts from the data; the EMG and EOG channels were otherwise removed from each EEG recording during pre-processing. For more details on the materials and design of EEG acquisition see Appendix D-2.

\section{EEG Analysis}

EEG analysis was conducted on the 3 minutes of eyes opened data digitized at a sampling frequency of $250 \mathrm{~Hz}$. Eye blinks and other stereotypical artifacts were removed by independent component analysis (ICA) via EEGLAB [24] and the Infomax algorithm [25] (blinking and 
lateral eye movements). Statistically defined artifact rejection was then carried out with the FASTER method removing segments based on extreme deviations of amplitude and variance from the mean [26].

After carefully removing artifacts the data were analyzed using Neurophysiological Biomarker Toolbox (NBT) in MATLAB 2018b software [27,28]. The EEG signal was rereferenced to current-source density (CSD) montage and band-pass filtered between 1 and 45hz, and fast-Fourier transform was used to generate five frequency bands: 1-4 Hz (delta), 4-8 Hz (theta), $8-12 \mathrm{~Hz}$ (alpha), $12-30 \mathrm{~Hz}$ (beta), and 30-45 Hz (gamma) [29]. Values for absolute power were calculated for each frequency band at each electrode. Then, the theta/beta ratio was computed by taking the theta amplitude from each electrode and each subject and dividing it by the corresponding beta amplitude.

In line with previous research the following analyses were conducted on the TBR at electrodes $\mathrm{Fz}$ and $\mathrm{Cz}$. Therefore, the use of Fz and $\mathrm{Cz}$ in our analyses enabled us to complete the most accurate replication and comparison of our data to the current literature.

\section{Clustering Analysis}

In line with the findings from Bussalb et al [1] we hypothesized that there would be 2 unique clusters of high and low $\mathrm{TBR}$ at $\mathrm{Fz} / \mathrm{Cz}$ in our sample. Therefore we used a k-means clustering method in SPSS 25.0 which allowed us to set a determined number of clusters $(\mathrm{k}=2)$. As k-means clustering is an unsupervised machine learning technique this was completed using unlabeled data. K-means clustering estimates Euclidian distances between subjects and potential clusters based on similarities in distance from the centroid. The centroid is the position of the center in the n-dimensional space of the TBR at Fz and $\mathrm{Cz}$ of all subjects. The clustering algorithm assigned each subject to exactly one of two TBR clusters based on each individuals 
mean and its distance from the centroid. After the machine assigned each subject to one of two clusters, each cluster centroid was recalculated by taking a grand average of all subjects. The process of assigning subjects to clusters was then repeated using these new optimized centroids. The assignment of clusters was further repeated for up to 10 iterations (reached optimal limit at 7 iterations), each time with a new starting point. Now, the final two clusters were defined by those which had the least sum of squared distances of all subjects to their corresponding centroid. Finally, we were provided with a high $(\mathrm{n}=79)$ and low TBR $(\mathrm{n}=110)$ cluster. This procedure concluded with an ANOVA comparing the TBR clusters.

\section{Clustering Validation}

The derived clusters were then validated using a supervised machine learning technique. In order to determine the validity of the clusters we aimed to create a support vector machine learning model that could classify the derived clusters with high accuracy. As such, a support vector non-probabilistic binary linear kernel classification analysis was conducted using open sourced java based software called Rapid Miner Studio 9.5 [30]. The analysis consisted of 189 rows and 3 columns (ie., k-means cluster assignment, and TBR at each Fz and $\mathrm{Cz}$ for all 189 participants). The cluster column was used to predict the otherwise unlabeled TBR values at $\mathrm{Fz}$ and $\mathrm{Cz}$.

The validation process began with the creation of a training and validation set; where the total data set was split into 60:40 training $(\mathrm{n}=113)$ and validation $(\mathrm{n}=76)$ set using automatic stratified sampling in a multiple hold-out performance calculation. The training set is labeled data which allowed the machine to learn which values appear to belong to which cluster. The validation set is unlabeled and therefore the machine predicted the cluster assignment based on only the numerical TBR values. Next, automatic feature selection using a multi-objective 
evolutionary algorithm was completed to derive the model with the best feature sets taking into account complexity of the model versus the error rate. Then the training set was trained using cost-sensitive scoring whereby a 10 -fold multiplier generated 10 artificial datapoints for each real datapoint that was being predicted. The distributions of the artificial datapoints were then used to define the confidence in terms of their proximity to the real value being predicted. These confidences were then averaged and used to derive the expected cost (potential error). Values with the lowest costs were used to define the ultimate prediction within the training set. For the validation set the known trained values were applied to the unlabeled hold out set. Then the optimal feature set was applied to the training data and the validation model before applying the final prediction model. Lastly, the final prediction model was derived by once again applying the aforementioned 10 -fold cost-sensitive scoring method to the validation set based on the optimal training set.

Finally, an analysis of variance was conducted to demonstrate that the clusters were significantly different from one another.

\section{Statistical Analysis}

\section{TBR and ADHD Analysis}

The aim of the following statistical analysis was to investigate any relationship that might exist between the low and high TBR clusters with ADHD. Although our sample was generally considered to be typically developing, all subjects were evaluated for ADHD. We investigated subjects' TBR in the inattentive subtype, hyperactive/impulsive subtype, and overall ADHD. As such, we repeated the same analysis threefold. We started by creating 12 derived variables including: $1,2,3,4$ or more inattentive symptoms, $1,2,3,4$ or more hyperactive/impulsive symptoms, $1,2,3,4$ or more of any $\mathrm{ADHD}$ symptom. This allowed us to calculate the frequency 
of subjects with 1-4 or more symptoms in each high and low TBR cluster. A series of chi-squares were conducted to investigate if any significant proportion of the high or low TBR cluster could be explained by any of these 12 derived ADHD variables. Effect sizes for each chi square were also computed.

In addition to these derived variables we measured each symptom on a continuous scale. To further test our hypothesis if there was any relationship between TBR and ADHD the mean score for the inattentive, hyperactive, and combined subtypes were compared between the high and low TBR cluster using independent t-tests. Independent sample Bayesian tests were subsequently calculated to demonstrate not only that the clusters do not differ by ADHD, but that they were actually statistically similar $[31,32]$.

A secondary analysis was conducted to investigate the relationship between sex and high vs low TBR cluster. Similar to the previous analysis, chi-squares were conducted to see if any significant proportion of the high or low TBR cluster could be explained sex. Finally, as a tertiary outcome we repeated our initial analysis that was conducted on the 12 derived ADHD variables, and 3 continuous variables by replacing TBR high/low with sex male/female.

\section{Results}

\section{Clustering}

The primary goals of our analysis were to cluster all of the subjects based on their TBR at Fz and Cz into two clusters, to derive a TBR threshold, and to investigate if TBR clusters had any relationship with ADHD. In the following paragraphs I will outline the success of our clustering analysis, and demonstrate that TBR has no relationship with ADHD. 
Our clustering analysis yielded a high $(\mathrm{n}=79, \mu=4.17, \mathrm{SD}=.56$, range $=3.56-6.04,40$ males $[\mu=4.2], 39$ females $[\mu=4.14])$ and low $(\mathrm{n}=110, \mu=2.91, \mathrm{SD}=.44$, range $=1.79-3.89$, 57 males $[\mu=2.86], 53$ females $[\mu=2.96])$ TBR cluster with an averaged threshold of 3.725 . See Figure 1a)-1e). This threshold is strikingly close to the initial threshold of 3.706 found by Bussalb et al [1]. Bussalb found that $35 \%$ of their data fell above the cut off, and similarly $34 \%$ of our data falls above the same threshold (Table 3).

Table 3. TBR thresholds from the literature compared to the present sample

\begin{tabular}{|l|l|l|l|}
\hline Threshold & $\begin{array}{l}\text { Threshold value } \\
\text { (adapted from Bussalb } \\
\text { and previous } \\
\text { literature) }\end{array}$ & $\begin{array}{l}\text { \% of observations } \\
\text { (patients) above the } \\
\text { threshold from } \\
\text { Bussalb et al. }\end{array}$ & $\begin{array}{l}\text { \% of observations } \\
\text { (healthy controls) } \\
\text { above the threshold } \\
\text { from the present study }\end{array}$ \\
\hline BGMM Threshold & 3.706 & $36 \%$ & $34 \%$ \\
\hline $35 \%$ Threshold & 3.752 & $35 \%$ & $33 \%$ \\
\hline Ward Threshold & 4.032 & $29 \%$ & $22 \%$ \\
\hline $10 \%$ Threshold & 4.129 & $28 \%$ & $16 \%$ \\
\hline 4.5 Threshold & 4.5 & $24 \%$ & $9 \%$ \\
\hline 5 Threshold & 5 & $19 \%$ & $3 \%$ \\
\hline
\end{tabular}

The ANOVA conducted at the end of the clustering analysis revealed that these clusters were significantly different $F(1,187)=307.68, p<0.00001$, partial $\eta^{2}=.622$ indicating that the clustering analysis was very successful in splitting the TBR data. The support vector machine learning analysis validated the clusters through the creation of a prediction model. This approach was able to predict which cluster a random TBR value belonged to with $90.9 \%$ accuracy, sensitivity $80.4 \%$, and specificity $100 \%$. 
Figure 1a. Histogram of subjects in the high and low TBR clusters

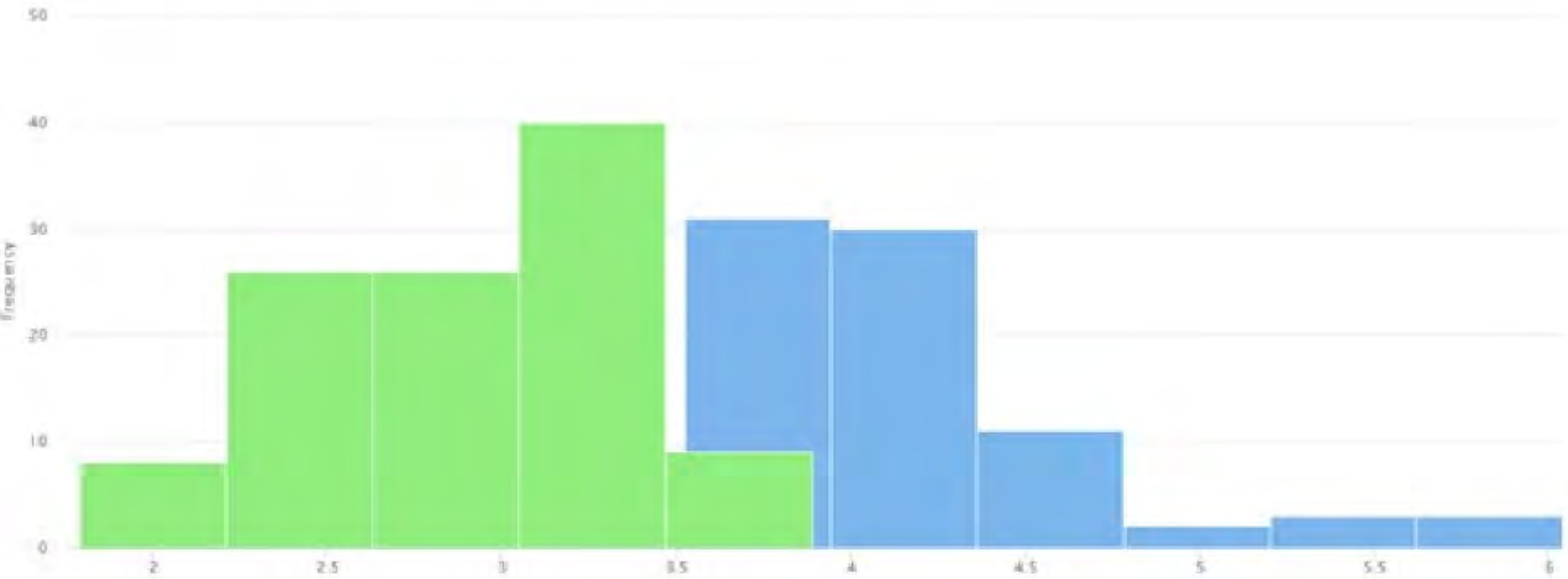

Figure 1b. Overlay of the high and low TBR cluster distributions

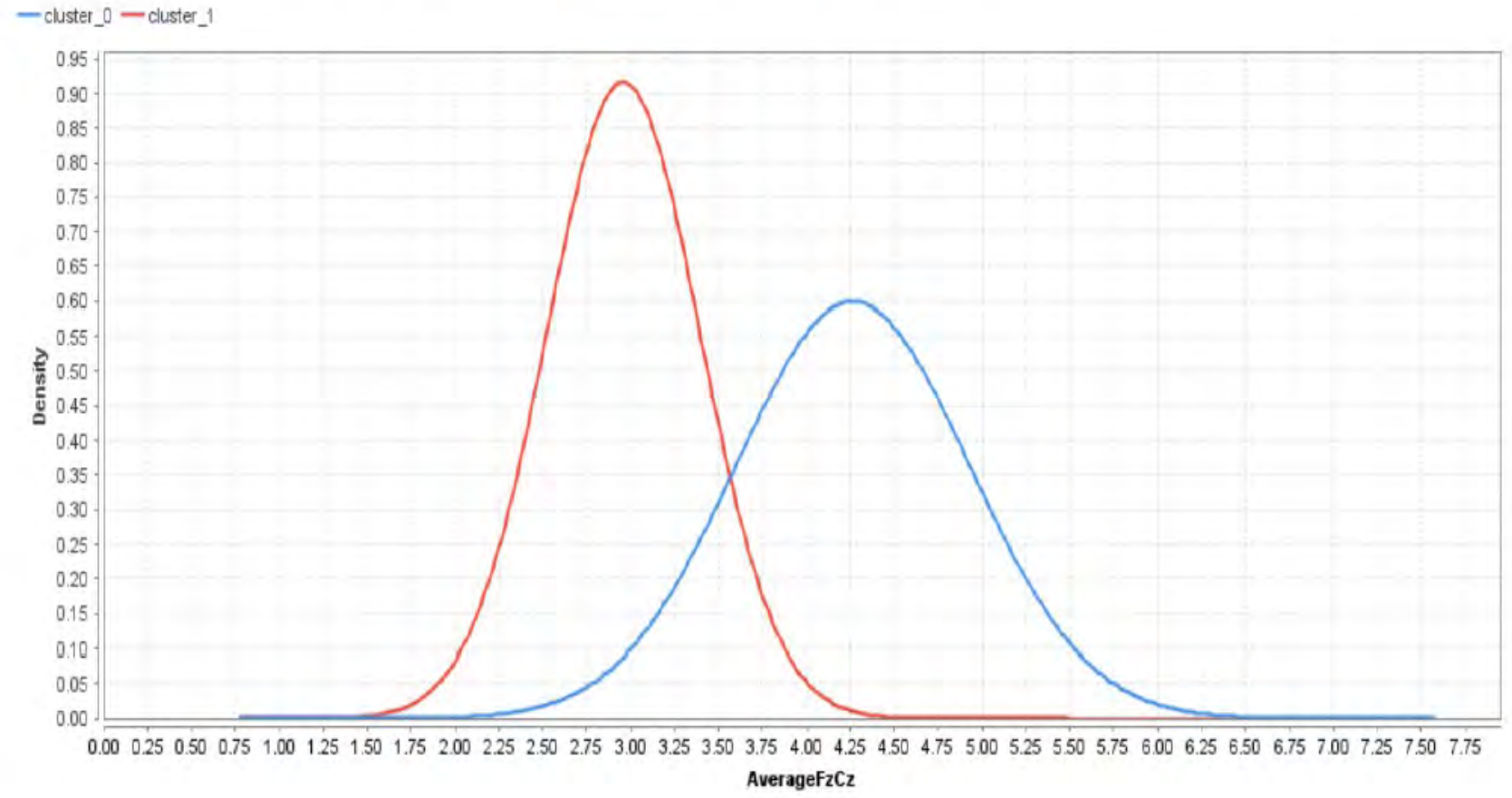


Figure 1c. Histogram of subjects in the high and low TBR clusters adopted from Bussalb for comparison. Note: their values should be squared for comparison.

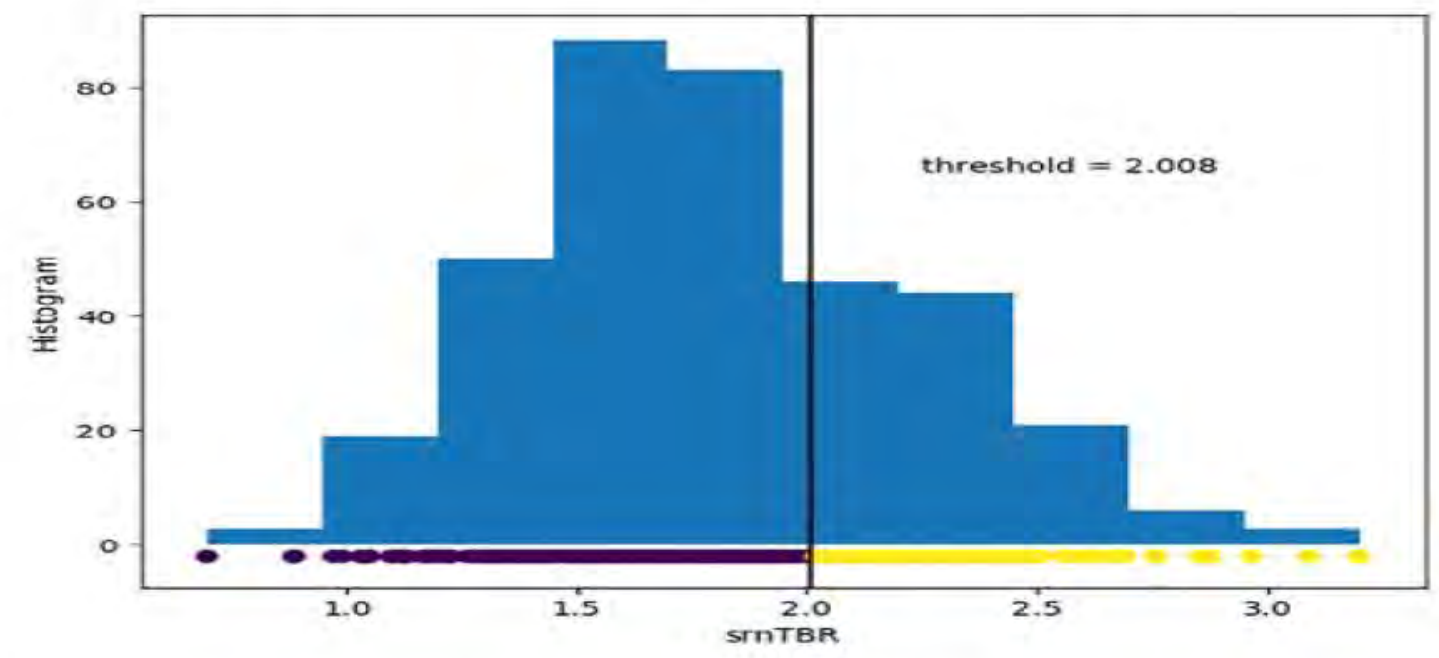

Figure 1d. Overlay of the high and low TBR cluster distributions adopted from Bussalb for comparison. Note: their values should be squared for comparison.

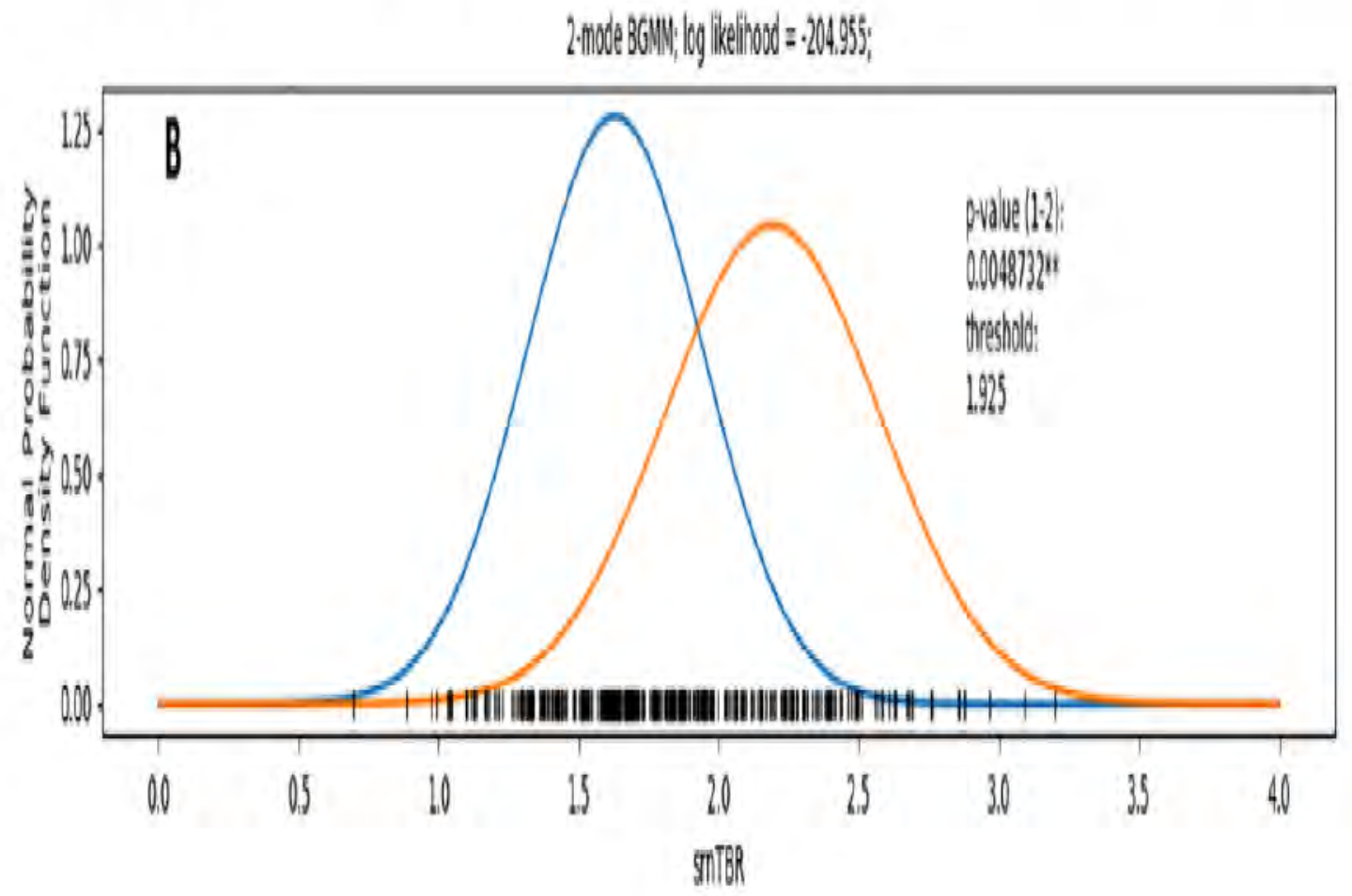


Figure 1e. Boxplot of high and low TBR clusters averaged at $\mathrm{Fz}$ and $\mathrm{Cz}$

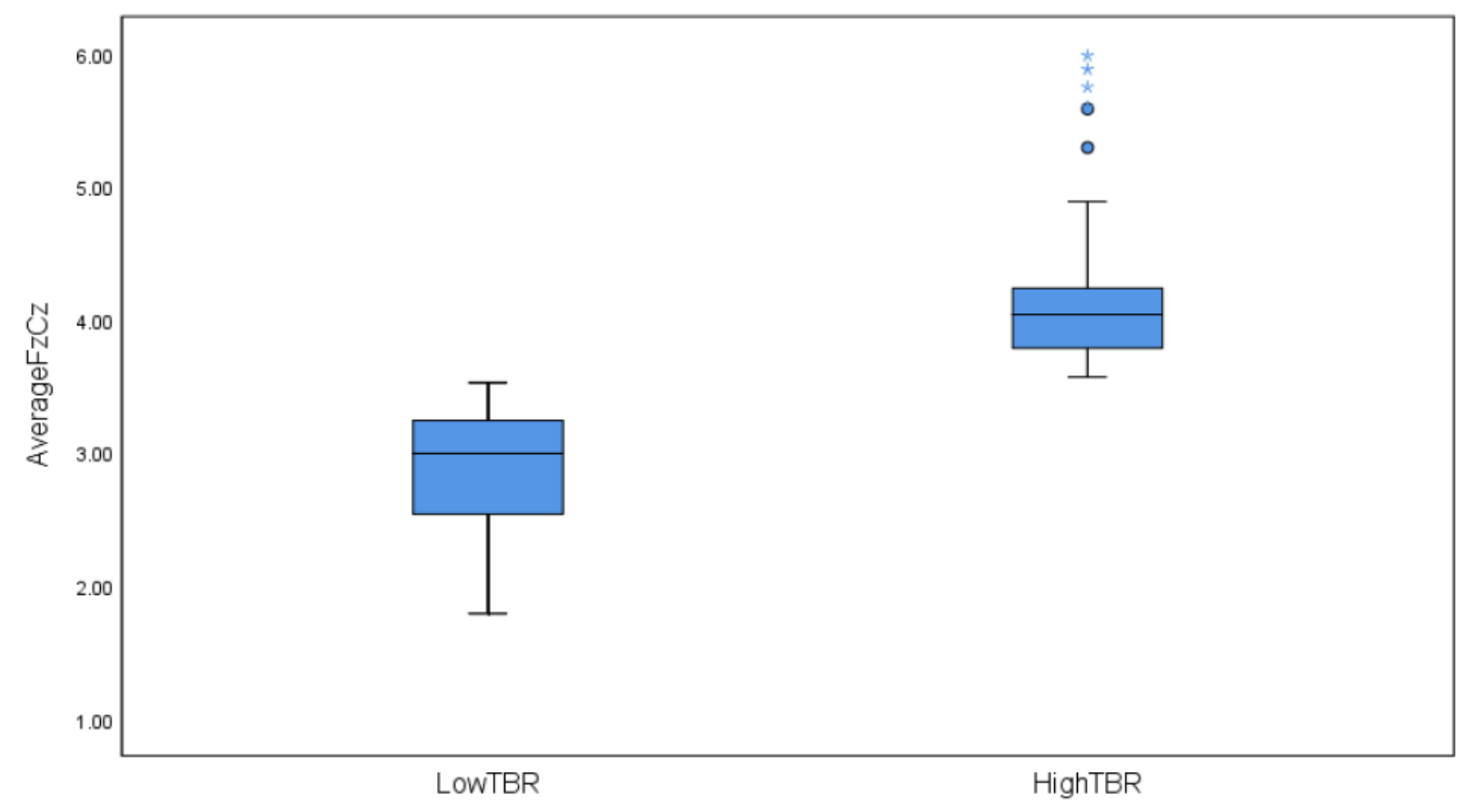

\section{ADHD and TBR Clusters}

After generating and validating the clusters the next step was to determine if there was any relationship between the high and low TBR clusters with ADHD symptomology. To do this a series of 12 chi-squares were conducted based on subjects with a number of ADHD symptoms of 1-4 or more. 11 of the chi-square analyses did not reveal any significant differences in proportion of subjects with inattentive, hyperactive, or overall ADHD symptoms (Table 4 and Figure 2a-c). However, the chi-square for 1 or more ADHD symptoms revealed that there were significantly more children with 1 or more ADHD symptom in the low TBR cluster. A complete table illustrating the frequency of symptoms by subtype is also provided (Table 5). 
Table 4. Chi-square comparisons for 1-4+ inattentive, hyperactive, and overall ADHD symptoms between high and low TBR cluster

\begin{tabular}{|c|c|c|c|c|c|c|c|c|c|}
\hline \multicolumn{10}{|c|}{ Cluster } \\
\hline Subtype & \multicolumn{3}{|c|}{ Inattentive } & \multicolumn{3}{|c|}{ Hyperactive/Impulsive } & \multicolumn{3}{|c|}{ ADHD Overall } \\
\hline $\begin{array}{l}\text { Symptom } \\
\text { Frequency }\end{array}$ & $\mathrm{X}_{1,187}^{2}$ & $\eta_{\text {partial }}$ & $\begin{array}{c}\mathrm{P} \\
\text { Value }\end{array}$ & $\mathrm{X}_{1,187}^{2}$ & $\eta_{\text {partial }}$ & $\begin{array}{c}\mathrm{P} \\
\text { Value }\end{array}$ & $\mathrm{X}_{1,187}^{2}$ & $\eta_{\text {partial }}$ & $\begin{array}{c}\mathrm{P} \\
\text { Value }\end{array}$ \\
\hline $1+$ & .298 & .040 & .349 & 1.087 & .076 & .188 & 5.688 & .173 & .017* \\
\hline $2+$ & .236 & .035 & .380 & .964 & .071 & .212 & 1.578 & .091 & .209 \\
\hline $3+$ & .003 & .004 & .559 & 1.918 & .101 & .119 & .077 & .020 & .781 \\
\hline $4+$ & .418 & .047 & .354 & .907 & .069 & .242 & .010 & .007 & .919 \\
\hline
\end{tabular}

Figure 2a. Population pyramid count of inattentive ADHD symptoms in subjects from the high and low TBR clusters

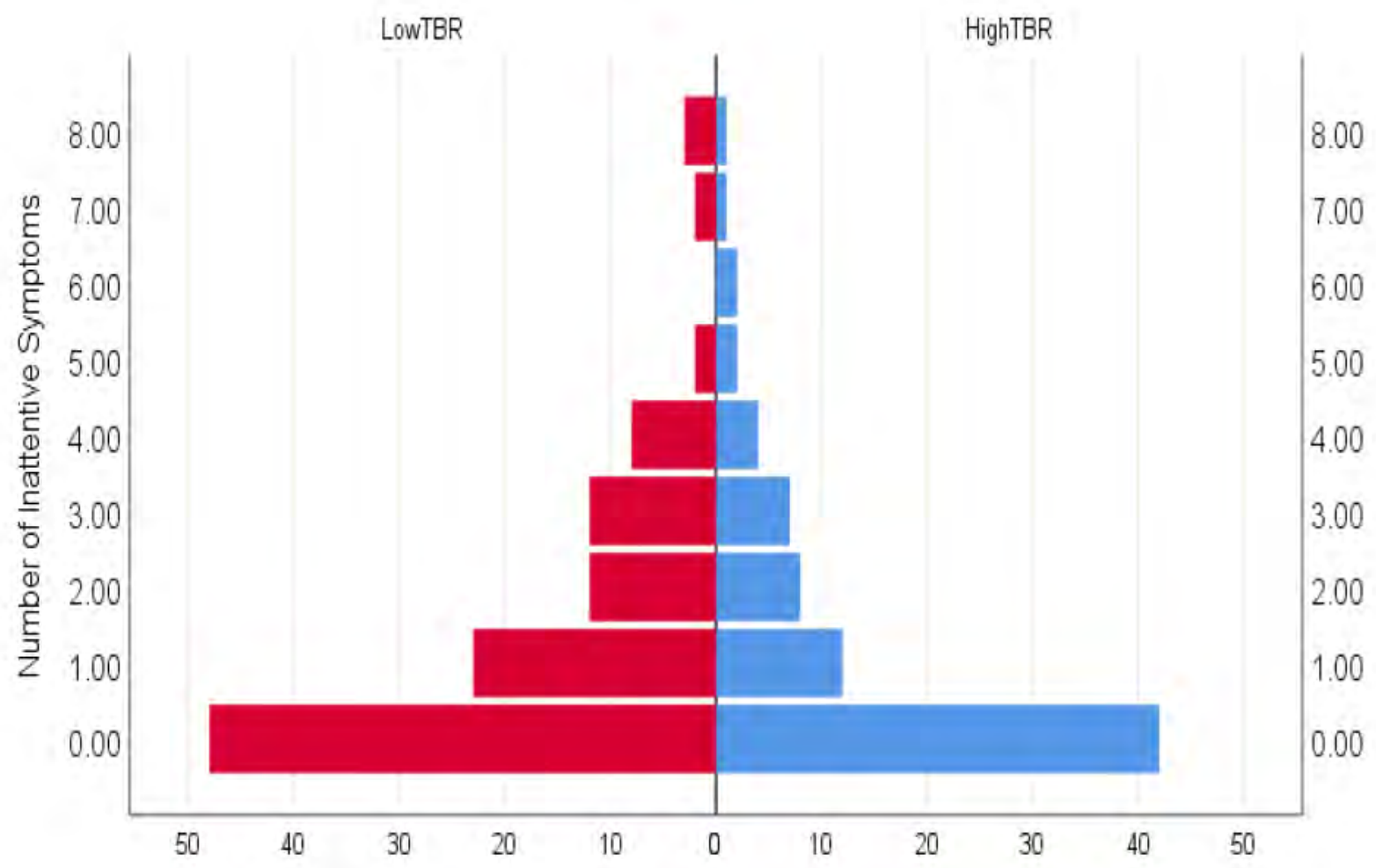


Figure 2b. Population pyramid count of hyperactive ADHD symptoms in subjects from the high and low TBR clusters

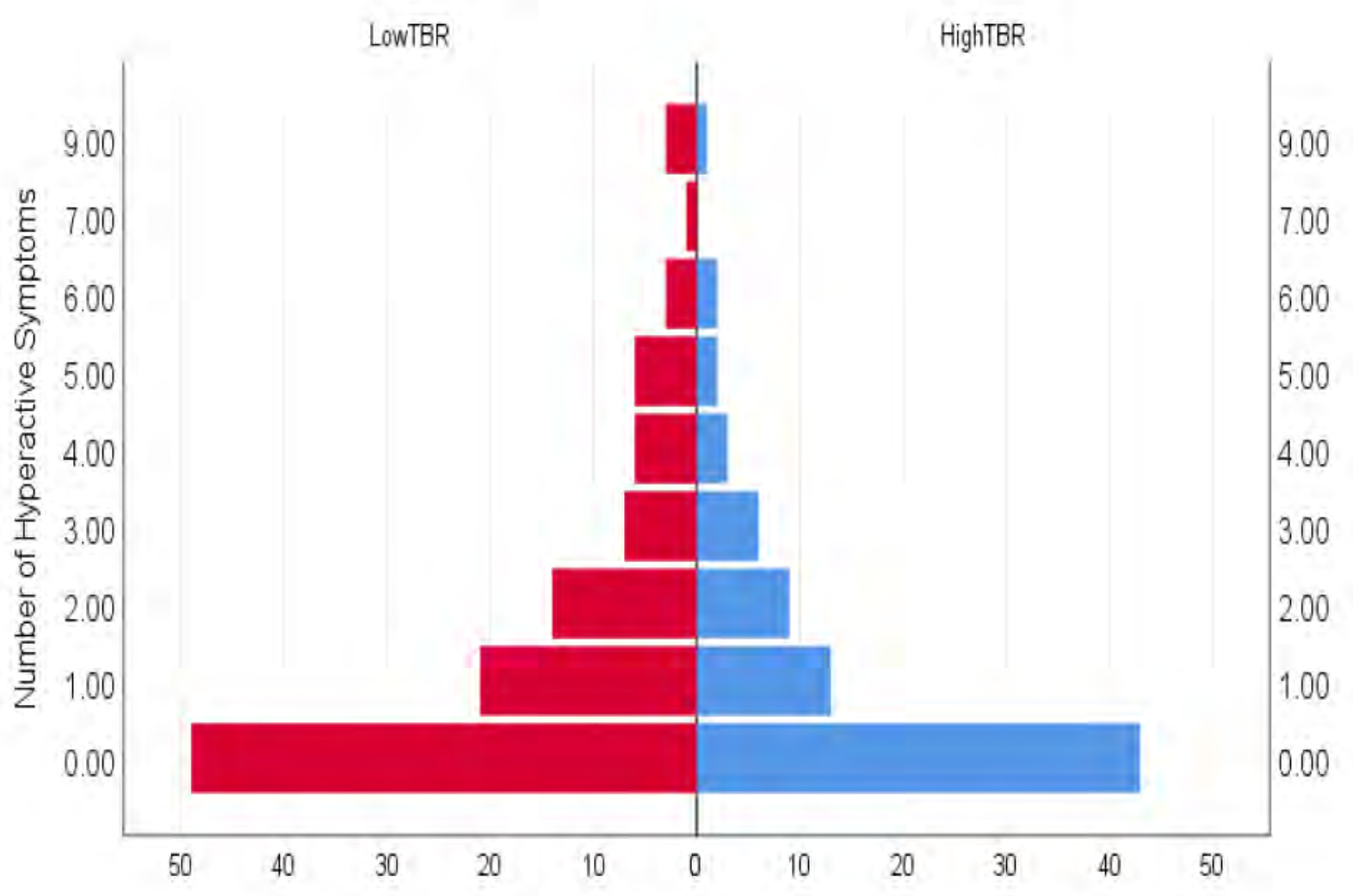


Figure 2a. Population pyramid count of overall ADHD symptoms in subjects from the high and low TBR clusters

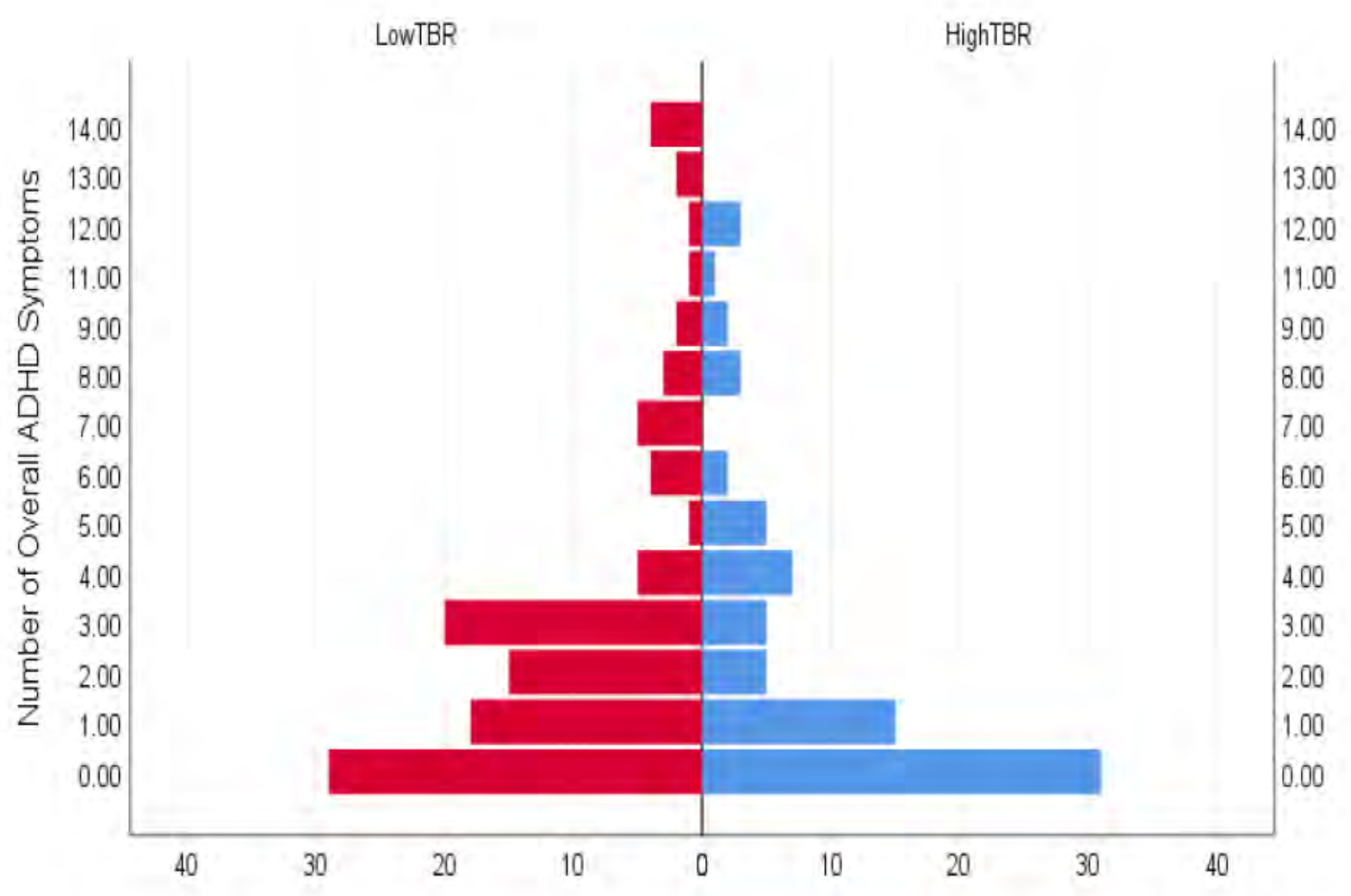


Table 5. Symptom counts for inattentive, hyperactive, and overall ADHD symptoms

\begin{tabular}{|l|c|c|c|c|c|c|c|c|c|}
\hline $\begin{array}{l}\text { Sympto } \\
\text { ms }\end{array}$ & \multicolumn{3}{|c|}{ Inattentive } & \multicolumn{3}{|c|}{ Hyperactive/Impulsive } & \multicolumn{3}{c|}{ ADHD Overall } \\
\hline $\begin{array}{l}\text { Count } \\
\text { er }\end{array}$ & $\begin{array}{c}\text { Numb } \\
\text { nt }\end{array}$ & $\begin{array}{c}\text { Cumulati } \\
\text { ve }\end{array}$ & $\begin{array}{c}\text { Numb } \\
\text { er }\end{array}$ & $\begin{array}{c}\text { Perce } \\
\text { nt }\end{array}$ & $\begin{array}{c}\text { Cumulati } \\
\text { ve }\end{array}$ & $\begin{array}{c}\text { Numb } \\
\text { er }\end{array}$ & $\begin{array}{c}\text { Perce } \\
\text { nt }\end{array}$ & $\begin{array}{c}\text { Cumulati } \\
\text { ve }\end{array}$ \\
\hline 0 & 90 & 47.6 & 47.6 & 92 & 48.7 & 48.7 & 60 & 31.7 & 31.7 \\
\hline 1 & 35 & 18.5 & 66.1 & 34 & 18.0 & 66.7 & 33 & 17.5 & 49.2 \\
\hline 2 & 20 & 10.6 & 76.7 & 23 & 12.2 & 78.8 & 20 & 10.6 & 59.8 \\
\hline 3 & 19 & 10.1 & 86.8 & 13 & 6.9 & 85.7 & 25 & 13.2 & 73.0 \\
\hline 4 & 12 & 6.3 & 93.1 & 9 & 4.8 & 90.5 & 12 & 6.3 & 79.4 \\
\hline 5 & 4 & 2.1 & 95.2 & 8 & 4.2 & 94.7 & 6 & 3.2 & 82.5 \\
\hline 6 & 2 & 1.1 & 96.3 & 5 & 2.6 & 97.4 & 6 & 3.2 & 85.7 \\
\hline 7 & 3 & 1.6 & 97.9 & 1 & .5 & 97.9 & 5 & 2.6 & 88.4 \\
\hline 8 & 4 & 2.1 & 100.0 & - & - & - & 6 & 3.2 & 91.5 \\
\hline 9 & - & - & - & 4 & 2.1 & 100.0 & 4 & 2.1 & 93.7 \\
\hline 10 & - & - & - & - & - & - & - & - & - \\
\hline 11 & - & - & - & - & - & - & 2 & 1.1 & 94.7 \\
\hline 12 & - & - & - & - & - & - & 4 & 2.1 & 96.8 \\
\hline 13 & - & - & - & - & - & - & 2 & 1.1 & 97.9 \\
\hline 14 & - & - & - & - & - & - & 4 & 2.1 & 100.0 \\
\hline
\end{tabular}


Next, we investigated TBR along a continuous scale of ADHD. Three independent sample t-tests were conducted on the high and low clusters based on the continuous inattentive scale, hyperactive scale, and overall ADHD scale. The t-test analyses did not reveal any significant differences between subjects in the high TBR group versus the low TBR group for any subtype (Table 6). Moreover, three independent sample Bayesian analyses were also conducted in parallel. This analysis actually provided moderate to strong evidence that subjects' inattentive, hyperactive, and overall ADHD scores between the high and low TBR clusters were actually statistically similar (inattentive $=8.35$, hyperactive $=4.59$, overall $\mathrm{ADHD}=6.43$ ). In other words, there does not appear to be any relationship between the continuous ADHD variables and TBR. These results are further illustrated in Figure 2a)-c).

Table 6. T-tests and Bayes comparisons for averaged continuous inattentive, hyperactive, and overall ADHD scores for clusters

\begin{tabular}{|c|c|c|c|c|c|c|c|c|c|}
\hline Group & \multicolumn{3}{|c|}{ Inattentive } & \multicolumn{3}{c|}{ Hyperactive/Impulsive } & \multicolumn{3}{c|}{ ADHD Overall } \\
\hline $\begin{array}{c}\text { Continuous } \\
\text { Scale }\end{array}$ & $\mathrm{T}_{1,187}$ & Bayes & $\begin{array}{c}\mathrm{P} \\
\text { Value }\end{array}$ & $\mathrm{T}_{1,187}$ & Bayes & $\begin{array}{c}\mathrm{P} \\
\text { Value }\end{array}$ & $\mathrm{T}_{1,187}$ & Bayes & $\begin{array}{c}\mathrm{P} \\
\text { Value }\end{array}$ \\
\hline Cluster & .284 & 8.35 & .777 & 1.15 & 4.59 & .252 & .789 & 6.43 & .431 \\
\hline
\end{tabular}


Figure 3a. Boxplot for inattention continuous measurement in the high and low TBR clusters

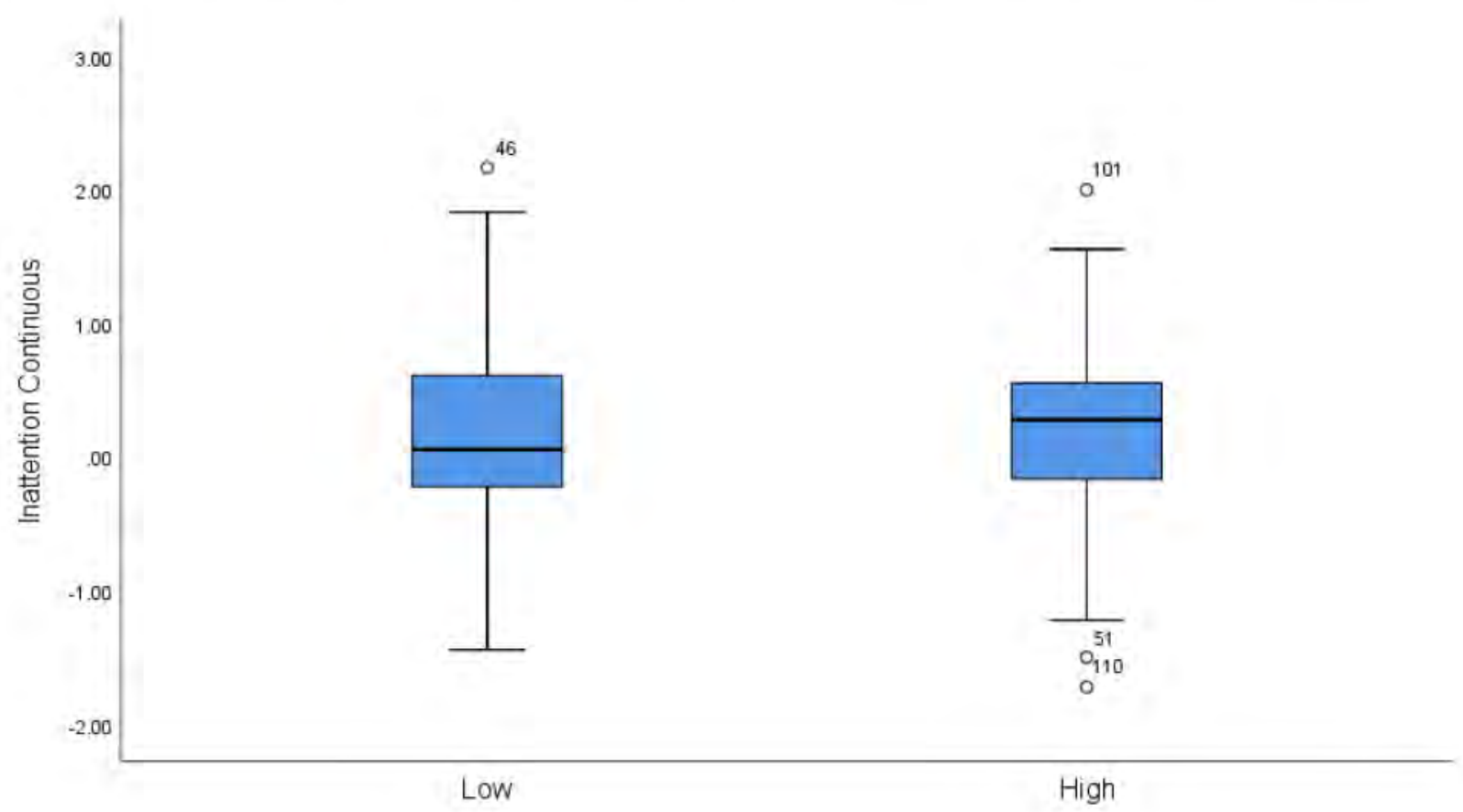

Figure 3b. Boxplot for hyperactive continuous measurement in the high and low TBR clusters

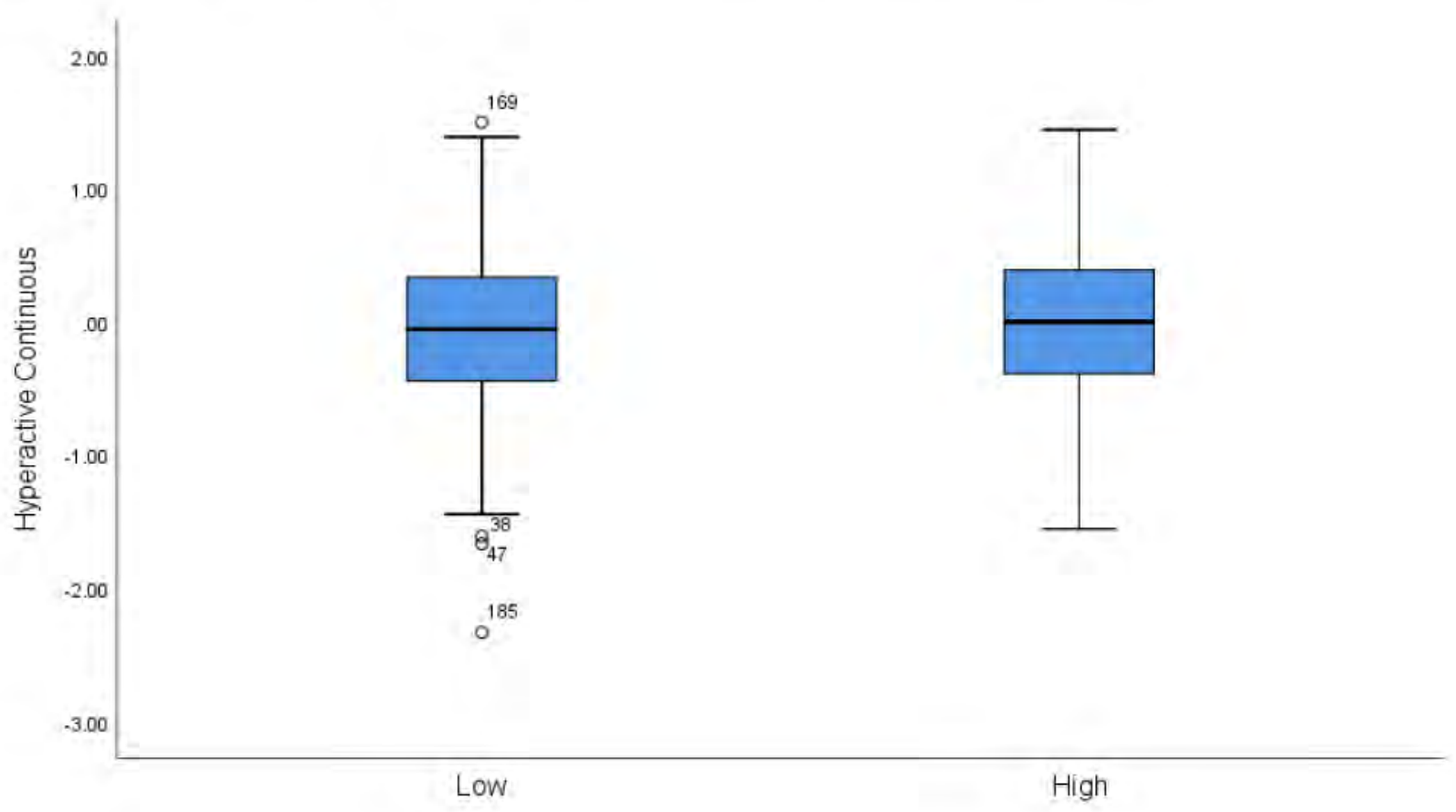


Figure 3c. Boxplot for overall ADHD continuous measurement in the high and low TBR clusters

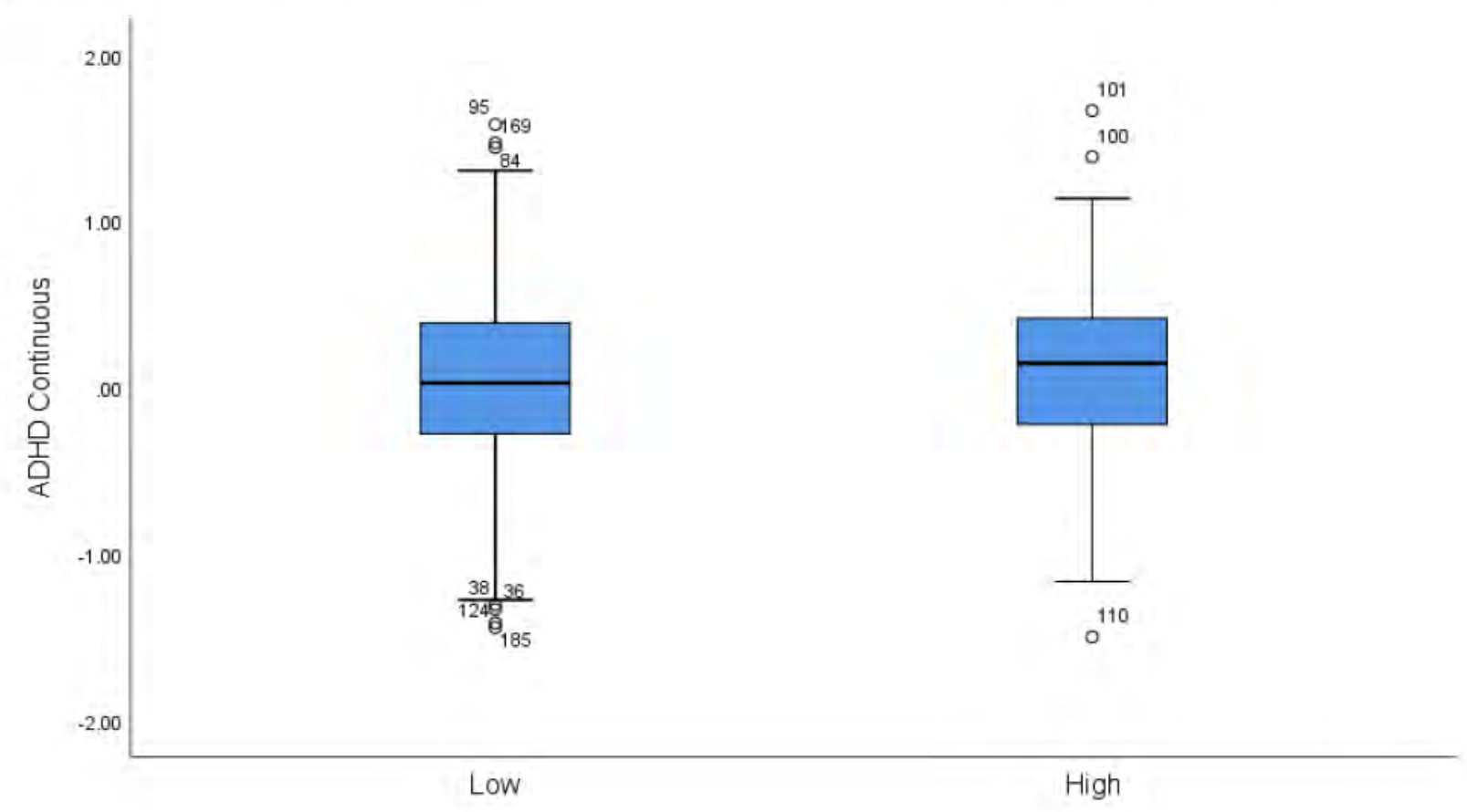

\section{Sex and TBR Clusters}

The next step in our analysis sought to investigate if there was some other variable that could explain the high and low TBR clusters. We therefore investigated the most salient biological factor in our sample, sex. As such, another chi-square was conducted but this time between clusters for males and females. Sex $\left(\mathrm{X}^{2}(1, \mathrm{~N}=187)=.111, \mathrm{p}=.427, \eta_{\text {partial }}=.024\right)$ could not explain any significant proportion of the high or low TBR clusters. The similarities between males and females in the high and low clusters are further illustrated in Figure 3. 
Figure 4. Boxplot of male and female $\mathrm{TBR}$ averaged at $\mathrm{Fz}$ and $\mathrm{Cz}$

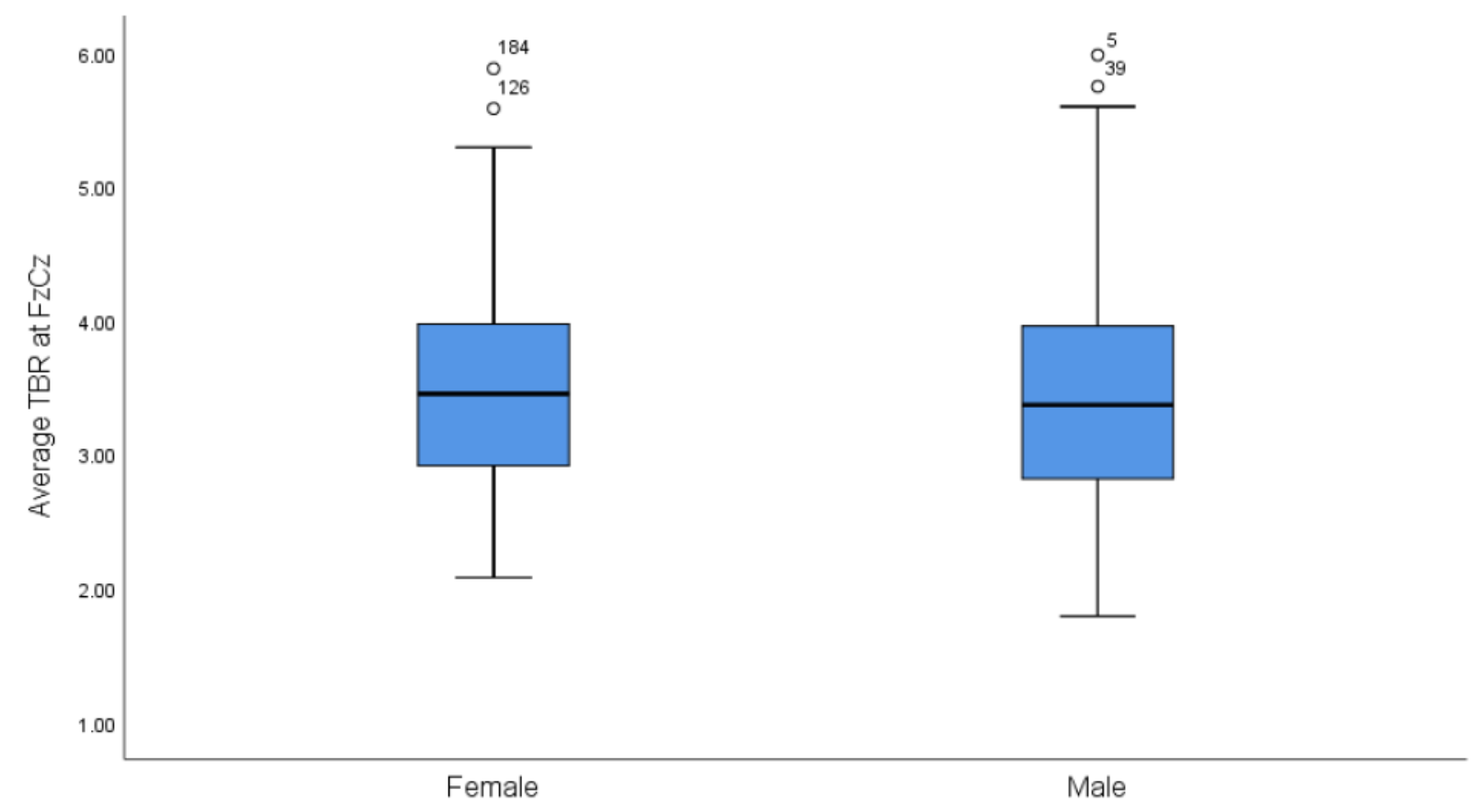

\section{Sex and ADHD}

The final step in our analysis moved away from the TBR clusters entirely and independently focused on investigating if there was any relationship between sex and ADHD symptom counts or continuous scales. Once again, the chi-square analyses from the previous sections were repeated but this time for sex males/females instead of TBR high/low. The results of this chi-square analyses are illustrated in (Table 7). This revealed that there were significantly more males than females with 3 or more inattentive symptoms, and 4 or more overall ADHD symptoms. 
Table 7. Chi-square comparisons for 1-4+ inattentive, hyperactive, and overall ADHD symptoms between males and females

\begin{tabular}{|c|c|c|c|c|c|c|c|c|c|}
\hline \multicolumn{10}{|c|}{ Sex } \\
\hline Subtype & \multicolumn{3}{|c|}{ Inattentive } & \multicolumn{3}{|c|}{ Hyperactive/Impulsive } & \multicolumn{3}{|c|}{ ADHD Overall } \\
\hline $\begin{array}{l}\text { Symptom } \\
\text { Frequency }\end{array}$ & $\mathrm{X}_{1,187}^{2}$ & $\eta_{\text {partial }}$ & $\begin{array}{c}\mathrm{P} \\
\text { Value }\end{array}$ & $\mathrm{X}^{2}{ }_{1,187}$ & $\eta_{\text {partial }}$ & $\begin{array}{c}\mathrm{P} \\
\text { Value }\end{array}$ & $\mathrm{X}^{2}{ }_{1,187}$ & $\eta_{\text {partial }}$ & $\begin{array}{c}\mathrm{P} \\
\text { Value }\end{array}$ \\
\hline $1+$ & .587 & .056 & .444 & 2.38 & .112 & .123 & 1.158 & .078 & .282 \\
\hline $2+$ & .833 & .066 & .361 & 2.78 & .121 & .095 & .313 & .041 & .576 \\
\hline $3+$ & 4.1 & .147 & $.043^{*}$ & .903 & .069 & .342 & 1.746 & .096 & .186 \\
\hline $4+$ & 1.1 & .076 & .294 & 2.63 & .118 & .105 & 4.097 & .147 & $.043^{*}$ \\
\hline
\end{tabular}

Lastly, three independent sample t-tests were conducted between males and females based on the continuous inattentive scale, hyperactive scale, and overall ADHD scale (Table 8). Females demonstrated significantly greater strengths (ie., protective factors) against ADHD in the overall ADHD condition, and was nearly significant for both inattentive and hyperactive subtypes. Moreover, three independent sample Bayesian analyses were also conducted in parallel to measure if males or females were the same along these continuous ADHD variables. This analysis failed to provide evidence for the null hypothesis, therefore indicating that the groups appear to differ based on ADHD. These results are further illustrated in Figure 4a)-c). 
Table 8. T-tests and Bayes comparisons for averaged continuous inattentive, hyperactive, and overall ADHD scores for sex

\begin{tabular}{|c|c|c|c|c|c|c|c|c|c|}
\hline Group & \multicolumn{3}{|c|}{ Inattentive } & \multicolumn{3}{c|}{ Hyperactive/Impulsive } & \multicolumn{3}{c|}{ ADHD Overall } \\
\hline $\begin{array}{c}\text { Continuous } \\
\text { Scale }\end{array}$ & $\mathrm{T}_{1,187}$ & Bayes & $\begin{array}{c}\mathrm{P} \\
\text { Value }\end{array}$ & $\mathrm{T}_{1,187}$ & Bayes & $\begin{array}{c}\mathrm{P} \\
\text { Value }\end{array}$ & $\mathrm{T}_{1,187}$ & Bayes & $\begin{array}{c}\mathrm{P} \\
\text { Value }\end{array}$ \\
\hline Sex & 1.932 & 1.47 & .055 & 1.841 & 1.73 & .067 & 2.096 & 1.076 & $\mathbf{. 0 3 7 ^ { * }}$ \\
\hline
\end{tabular}

Figure 5a. Boxplot for inattention continuous measurement in males and females

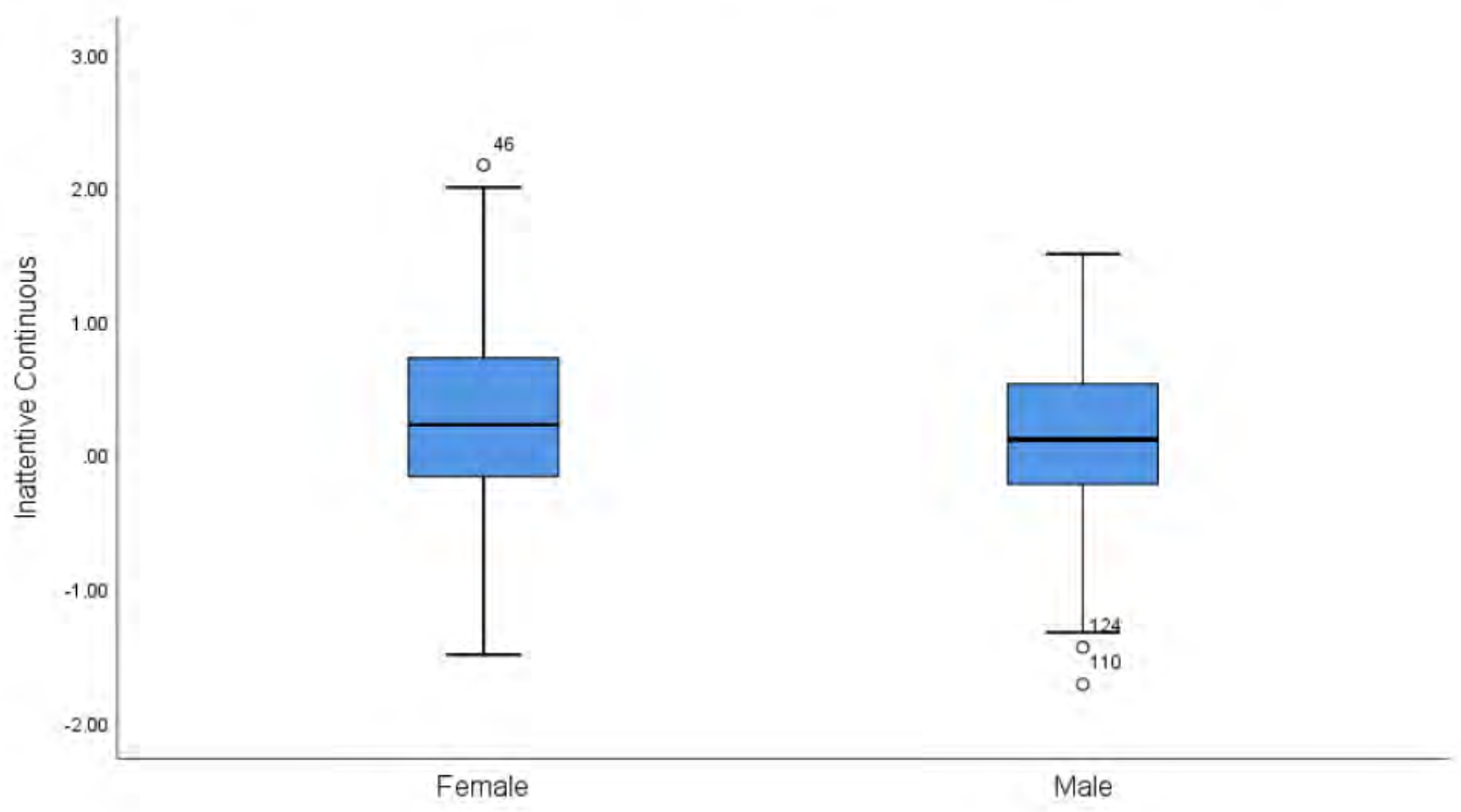


Figure 5b. Boxplot for hyperactive continuous measurement in males and females

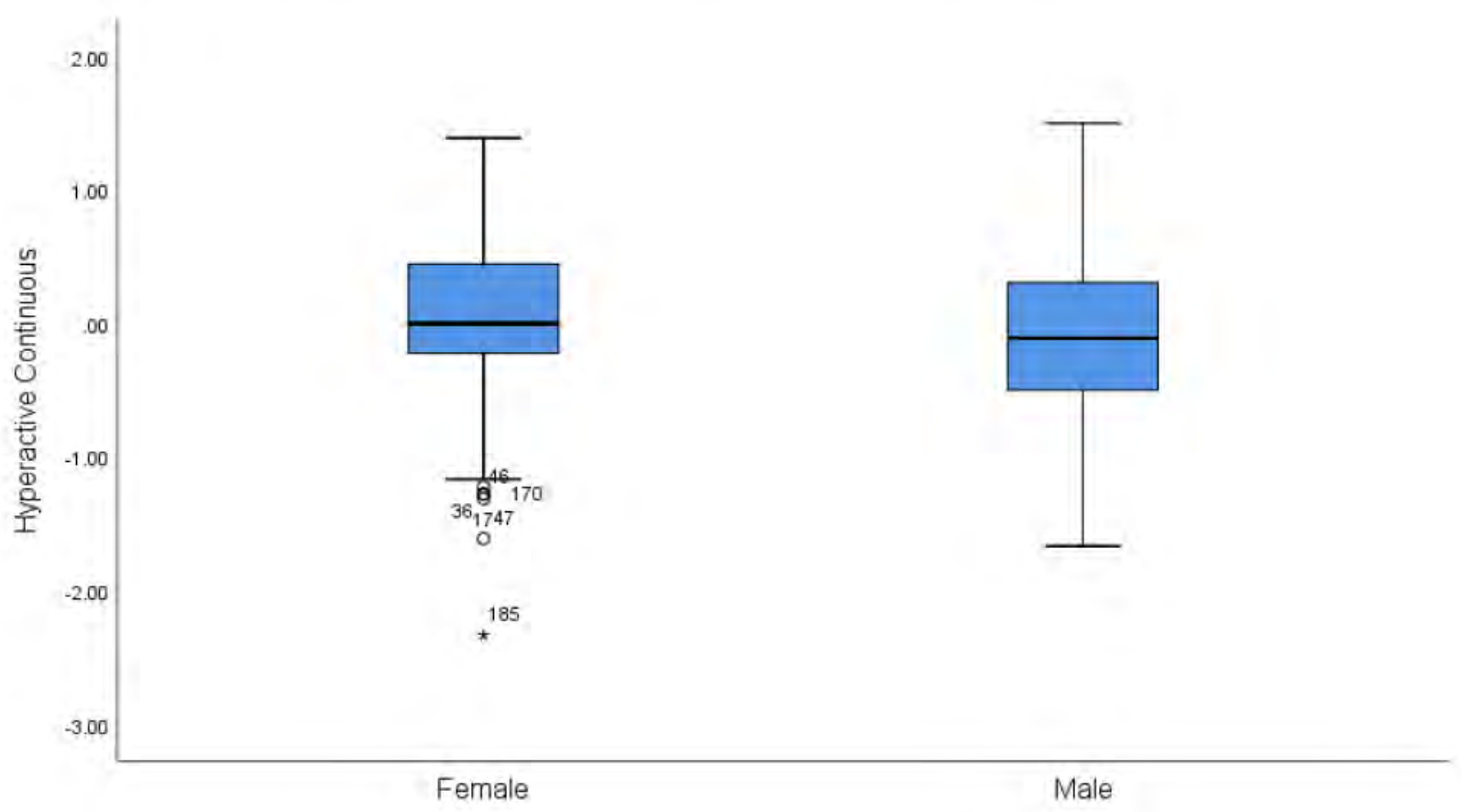

Figure 5c. Boxplot for overall ADHD continuous measurement in males and females

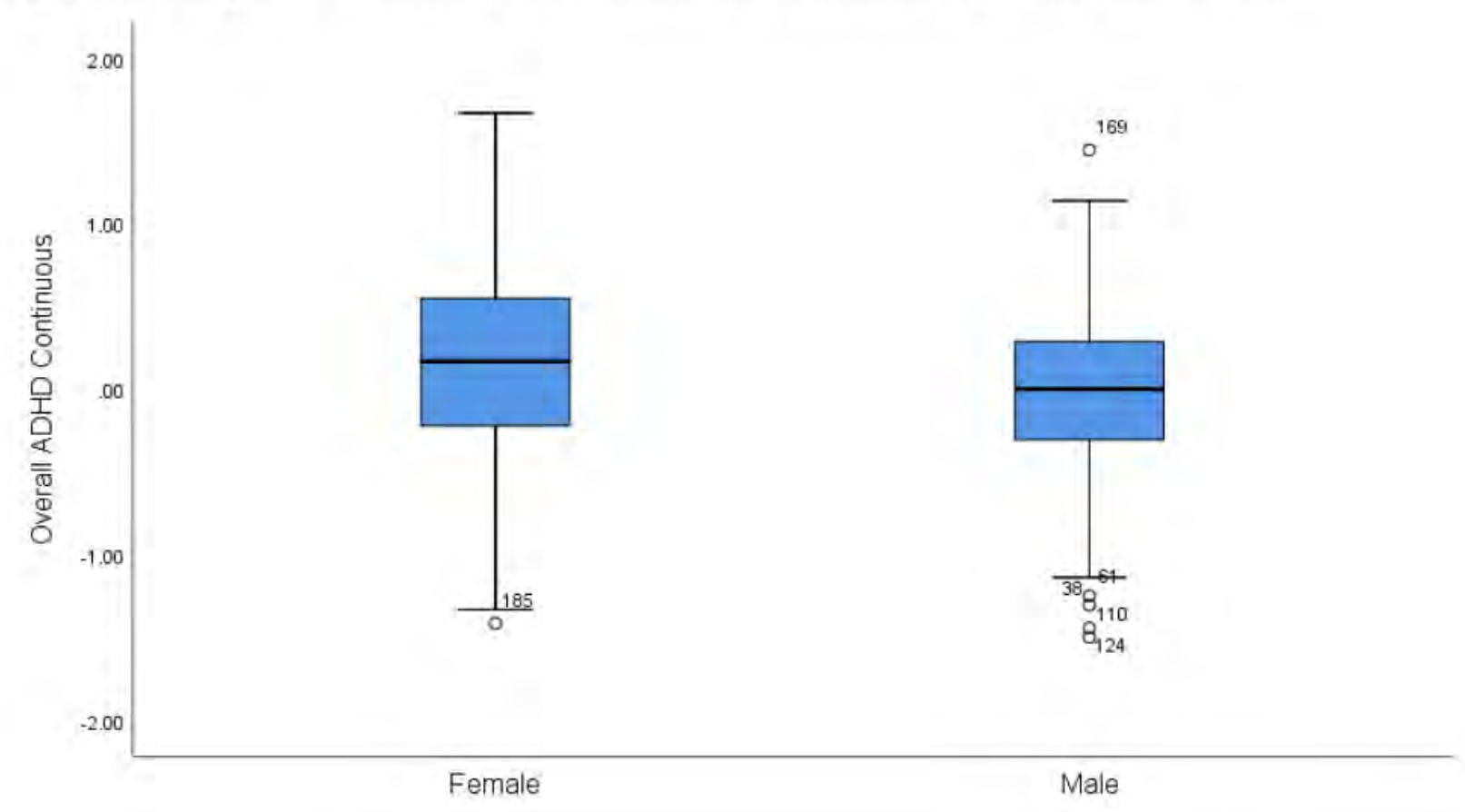




\section{Discussion}

In this study we found strong evidence for high and low clusters of TBR in a sample of 189 healthy children evaluated for ADHD; $60.4 \%$ of which had at least 1 inattentive or hyperactive symptom. These clusters yielded a TBR threshold that is strikingly similar to what Bussalb et al [1] reported in children with ADHD. 34\% of our healthy sample, compared to $36 \%$ of Bussalb's ADHD sample fell above the same threshold. The implication of this is that TBR thresholds cannot be considered reliable in $\mathrm{ADHD}$ if the same threshold is equally prevalent in healthy children. Moreover, Bussalb’s study found that clusters significantly differed by age, and they failed to report any relationship of ADHD with TBR clusters. Therefore, we improved upon their analysis by controlling for age and by investigating if TBR actually had any relationship with ADHD symptomology. To that end, we did not find any relationship between ADHD symptomology and the high and low TBR clusters. This further consolidates our conclusion from the previous analysis such that TBR cannot be considered a valid biomarker for ADHD.

As previous evidence has long demonstrated sex differences in $\mathrm{ADHD}$ [33-35] it was important to investigate this variable. We demonstrated that sex did not differ by cluster, but it did seem to play a role in ADHD symptomology such that there were significantly more males with $3+$ inattention, and $4+\mathrm{ADHD}$ symptoms compared to females. That being said the effect sizes were small (.147). This was further supported by our analysis of sex on ADHD strengths and weaknesses using our continuous ADHD variable. Females demonstrated significantly greater strengths (ie., protective factors) against $\mathrm{ADHD}$ in the in the overall $\mathrm{ADHD}$ condition, and was nearly significant for both inattentive and hyperactive subtypes. In general this is consistent with previous literature that boys are more prone to ADHD. Therefore, in the present sample, ADHD was better characterized by sex than TBR thresholds. 
Although we have demonstrated that TBR clusters do not have any relationship with ADHD, or sex, they still exist nonetheless. Since TBR clusters cannot be explained by ADHD it is possible that they may be explained by some other biological, behavioural, or environmental variable. Our future research aims to investigate this via an extension of the present analysis into the comorbidities of ADHD such as depression, anxiety, oppositional defiance disorder, or conduct disorder. That being said, a previous multi-site study $(\mathrm{n}=159)$ noted that TBR changes could not identify comorbidities of ADHD or alternative diagnoses [36].

It is also possible that TBR is not pathological at all, and indeed our current evidence seems to suggest this. Therefore, it may be more prudent to consider an alternative hypothesis that perhaps TBR is actually a more general index that varies within all children as a function of arousal $[37,38]$ or mind wandering [39]. Under this hypothesis it is proposed that TBR varies based on high and low attentional control. The advantage of this rationale is that it simultaneously allows for patients with ADHD and normal controls to have elevated high or low TBR based on attentional control during the EEG recording. For instance, van Son et al [39] demonstrated that TBR was significantly higher during mind wandering periods than during task oriented behavior. Therefore, as opposed to TBR being related to a pathological attentional disorder (ADHD), perhaps it is more generally related to the cognitive domain of attention and TBR simply decreases as a function of increased attentional control.

Our future research will elucidate this hypothesis by utilizing the odd-ball and go-no-go event-related potential (ERP) paradigm data also available from the Quebec Newborn Twin Study. The odd-ball and go-no-go ERP paradigms are each objective electrophysiological measures of impulsivity and attentional control. By combining the values from our TBR database 
with the ERP data we will be able to determine if attentional control can explain the differences in high and low TBR clusters.

The main potential limitation of our analysis is that we did not tailor our TBR values to individualized alpha frequencies such as Busalb [1] and others [40] have recently suggested. Saad et al [40] posits that computing TBR based on fixed frequency bands is 'inherently flawed'. It is possible that individualized TBR values may be more accurate representations of each subject's theta and beta oscillations, however the method we used to compute TBR is congruent with previous literature cited by Bussalb $[41,42]$. This was the same literature that Bussalb compared their thresholds to, and therefore our analysis and thresholds should be equally comparable.

The authors of this study believe that it is important to highlight that our analysis does not cast doubt on all NFB or tDCS in ADHD, but rather on TBR diagnostics and TBR related treatment in ADHD. tDCS and NFB protocols that were designed to target TBR need to be reconsidered; it is possible that there remains other viable treatment targets. For instance, according a 2019 meta-analyses [43] anodal modulation of the dorsolateral prefrontal cortex (but not the right inferior frontal gyrus) with tDCS in ADHD has demonstrated reasonable treatment efficacy on improving inhibitory control with small to medium effect sizes.

In conclusion, this study provided evidence that there is no relationship between TBR distributions in ADHD and its subtypes. Moreover, we provided evidence that the distribution of healthy subjects above the TBR threshold (34\%) is the same as in children with ADHD (36\%). This evidence questions the entire application of TBR in ADHD. In order to completely validate these results, future research should replicate the TBR clustering analysis in a sample of children 
with and without $\mathrm{ADHD}$ and analyze if the proportion of healthy children compared to ADHD in the high and low TBR clusters differ. 


\section{References}

1. Bussalb, A.; Collin, S.; Barthélemy, Q.; Ojeda, D.; Bioulac, S.; Blasco-Fontecilla, H.; Brandeis, D.; Purper Ouakil, D.; Ros, T.; Mayaud, L. Is there a cluster of high theta-beta ratio patients in attention deficit hyperactivity disorder? Clin. Neurophysiol. 2019, 130, 1387-1396, doi:10.1016/j.clinph.2019.02.021.

2. Polanczyk, G.; De Lima, M.S.; Horta, B.L.; Biederman, J.; Rohde, L.A. The worldwide prevalence of ADHD: A systematic review and metaregression analysis. Am. J. Psychiatry 2007, 164, 942-948, doi:10.1176/ajp.2007.164.6.942.

3. Polanczyk, G. V.; Willcutt, E.G.; Salum, G.A.; Kieling, C.; Rohde, L.A. ADHD prevalence estimates across three decades: An updated systematic review and metaregression analysis. Int. J. Epidemiol. 2014, 43, 434-442, doi:10.1093/ije/dyt261.

4. Saad, J.F.; Kohn, M.R.; Clarke, S.; Lagopoulos, J.; Hermens, D.F. Is the Theta/Beta EEG Marker for ADHD Inherently Flawed? J. Atten. Disord. 2018, 22, 815-826.

5. Gloss, D.; Varma, J.K.; Pringsheim, T.; Nuwer, M.R. Practice advisory: The utility of EEG theta/beta power ratio in ADHD diagnosis. Neurology 2016, 87, 2375-2379, doi:10.1212/WNL.0000000000003265.

6. Clarke, A.R.; Barry, R.J.; McCarthy, R.; Selikowitz, M. Children with attentiondeficit/hyperactivity disorder and comorbid oppositional defiant disorder: An EEG analysis. Psychiatry Res. 2002, doi:10.1016/S0165-1781(02)00137-3.

7. Snyder, S.M.; Hall, J.R. A meta-analysis of quantitative EEG power associated with attention-deficit hyperactivity disorder. J. Clin. Neurophysiol. 2006, doi:10.1097/01.wnp.0000221363.12503.78.

8. Arns, M.; Conners, C.K.; Kraemer, H.C. A Decade of EEG Theta/Beta Ratio Research in 
ADHD. J. Atten. Disord. 2013, 17, 374-383, doi:10.1177/1087054712460087.

9. Snyder, S.M.; Rugino, T.A.; Hornig, M.; Stein, M.A. Integration of an EEG biomarker with a clinician's ADHD evaluation. Brain Behav. 2015, 5, doi:10.1002/brb3.330.

10. Picken, C.; Clarke, A.R.; Barry, R.J.; McCarthy, R.; Selikowitz, M. The Theta/Beta Ratio as an Index of Cognitive Processing in Adults With the Combined Type of Attention Deficit Hyperactivity Disorder. Clin. EEG Neurosci. 2020, 51, 167-173, doi:10.1177/1550059419895142.

11. Denovo classification request for neuropsychiatric EEG-based assessment aid forADHD(NEBA) system; 2013. U.S. food anddrug Adm.

12. Ogrim, G.; Kropotov, J.; Hestad, K. The quantitative EEG theta/beta ratio in attention deficit/hyperactivity disorder and normal controls: Sensitivity, specificity, and behavioral correlates. Psychiatry Res. 2012, 198, 482-488, doi:10.1016/j.psychres.2011.12.041.

13. Liechti, M.D.; Valko, L.; Müller, U.C.; Döhnert, M.; Drechsler, R.; Steinhausen, H.C.; Brandeis, D. Diagnostic value of resting electroencephalogram in attention-deficit/ hyperactivity disorder across the lifespan. Brain Topogr. 2013, doi:10.1007/s10548-0120258-6.

14. Snyder, S.M.; Quintana, H.; Sexson, S.B.; Knott, P.; Haque, A.F.M.; Reynolds, D.A. Blinded, multi-center validation of EEG and rating scales in identifying ADHD within a clinical sample. Psychiatry Res. 2008, 159, 346-358, doi:10.1016/j.psychres.2007.05.006.

15. Loo, S.K.; Cho, A.; Hale, T.S.; McGough, J.; McCracken, J.; Smalley, S.L. Characterization of the Theta to Beta Ratio in ADHD: Identifying Potential Sources of Heterogeneity. J. Atten. Disord. 2013, 17, 384-392, doi:10.1177/1087054712468050.

16. Schönenberg, M.; Wiedemann, E.; Schneidt, A.; Scheeff, J.; Logemann, A.; Keune, P.M.; 
Hautzinger, M. Neurofeedback, sham neurofeedback, and cognitive-behavioural group therapy in adults with attention-deficit hyperactivity disorder: a triple-blind, randomised, controlled trial. The Lancet Psychiatry 2017, 4, 673-684, doi:10.1016/S22150366(17)30291-2.

17. Arns, M.; Vollebregt, M.A.; Palmer, D.; Spooner, C.; Gordon, E.; Kohn, M.; Clarke, S.; Elliott, G.R.; Buitelaar, J.K. Electroencephalographic biomarkers as predictors of methylphenidate response in attention-deficit/hyperactivity disorder. Eur. Neuropsychopharmacol. 2018, doi:10.1016/j.euroneuro.2018.06.002.

18. Arnold, L.E.; Lofthouse, N.; Hersch, S.; Pan, X.; Hurt, E.; Bates, B.; Kassouf, K.; Moone, S.; Grantier, C. EEG Neurofeedback for ADHD: Double-Blind Sham-Controlled Randomized Pilot Feasibility Trial. J. Atten. Disord. 2013, 17, 410-419, doi:10.1177/1087054712446173.

19. Bussalb, A.; Congedo, M.; Barthélemy, Q.; Ojeda, D.; Acquaviva, E.; Delorme, R.; Mayaud, L. Clinical and experimental factors influencing the efficacy of neurofeedback in ADHD: A meta-analysis. Front. Psychiatry 2019, 10, doi:10.3389/fpsyt.2019.00035.

20. Perone, S.; Palanisamy, J.; Carlson, S.M. Age-related change in brain rhythms from early to middle childhood: Links to executive function. Dev. Sci. 2018, doi:10.1111/desc.12691.

21. L. Eugene Arnold Double-Blind 2-Site Randomized Clinical Trial of Neurofeedback for ADHD. Clin. Regist. number NCT02251743; 2016. 2019.

22. Bioulac, S.; Purper-Ouakil, D.; Ros, T.; Blasco-Fontecilla, H.; Prats, M.; Mayaud, L.; Brandeis, D. Personalized at-home neurofeedback compared with long-acting methylphenidate in an european non-inferiority randomized trial in children with ADHD. BMC Psychiatry 2019, 19, doi:10.1186/s12888-019-2218-0. 
23. Segal, D.L. Diagnostic and Statistical Manual of Mental Disorders (DSM-IV-TR). In The Corsini Encyclopedia of Psychology; 2010.

24. Delorme, A.; Makeig, S. EEGLAB: an open source toolbox for analysis of single-trial EEG dynamics including independent component analysis. J. Neurosci. Methods 2004, doi:10.1016/j.jneumeth.2003.10.009.

25. Jung, T.P.; Makeig, S.; Humphries, C.; Lee, T.W.; Mckeown, M.J.; Iragui, V.; Sejnowski, T.J. Removing electroencephalographic artifacts by blind source separation. Psychophysiology 2000, doi:10.1017/S0048577200980259.

26. Nolan, H.; Whelan, R.; Reilly, R.B. FASTER: Fully Automated Statistical Thresholding for EEG artifact Rejection. J. Neurosci. Methods 2010, doi:10.1016/j.jneumeth.2010.07.015.

27. Simon-Shlomo Poil, Sonja Simpraga, Simon J. Houtman, K.L.-H. Neurophysiological Biomarker Toolbox.

28. MATLAB and Statistics Toolbox Release 2018b, The MathWorks, Inc.

29. Olsen, T. Current Source Density (CSD). 2019.

30. Mierswa, I.; Wurst, M.; Klinkenberg, R.; Scholz, M.; Euler, T. YALE: Rapid prototyping for complex data mining tasks. In Proceedings of the Proceedings of the ACM SIGKDD International Conference on Knowledge Discovery and Data Mining; 2006.

31. Dienes, Z.; Mclatchie, N. Four reasons to prefer Bayesian analyses over significance testing. Psychon. Bull. Rev. 2018, 25, 207-218, doi:10.3758/s13423-017-1266-z.

32. Rouder, J.N.; Speckman, P.L.; Sun, D.; Morey, R.D.; Iverson, G. Bayesian t tests for accepting and rejecting the null hypothesis. Psychon. Bull. Rev. 2009, 16, 225-237.

33. Hermens, D.F.; Kohn, M.R.; Clarke, S.D.; Gordon, E.; Williams, L.M. Sex differences in 
adolescent ADHD: Findings from concurrent EEG and EDA. Clin. Neurophysiol. 2005, 116, 1455-1463, doi:10.1016/j.clinph.2005.02.012.

34. Yallop, L.; Brownell, M.; Chateau, D.; Walker, J.; Warren, M.; Bailis, D.; Lebow, M. Lifetime Prevalence of Attention-Deficit Hyperactivity Disorder in Young Adults: Examining Variations in the Socioeconomic Gradient. CanJPsychiatry 2015, 6060, 432440.

35. Arnett, A.B.; Pennington, B.F.; Willcutt, E.G.; Defries, J.C.; Olson, R.K. Sex differences in ADHD symptom severity. J. Child Psychol. Psychiatry Allied Discip. 2015, 56, 632639, doi:10.1111/jcpp.12337.

36. Snyder, S.M.; Quintana, H.; Sexson, S.B.; Knott, P.; Haque, A.F.M.; Reynolds, D.A. Blinded, multi-center validation of EEG and rating scales in identifying ADHD within a clinical sample. Psychiatry Res. 2008, 159, 346-358, doi:10.1016/j.psychres.2007.05.006.

37. Barry, R.J.; Clarke, A.R.; McCarthy, R.; Selikowitz, M.; Rushby, J.A.; Ploskova, E. EEG differences in children as a function of resting-state arousal level. Clin. Neurophysiol. 2004, doi:10.1016/S1388-2457(03)00343-2.

38. Clarke, A.R.; Barry, R.J.; Karamacoska, D.; Johnstone, S.J. The EEG Theta/Beta Ratio: A marker of Arousal or Cognitive Processing Capacity? Appl. Psychophysiol. Biofeedback 2019, doi:10.1007/s10484-018-09428-6.

39. van Son, D.; De Blasio, F.M.; Fogarty, J.S.; Angelidis, A.; Barry, R.J.; Putman, P. Frontal EEG theta/beta ratio during mind wandering episodes. Biol. Psychol. 2019, doi:10.1016/j.biopsycho.2018.11.003.

40. Saad, J.F.; Kohn, M.R.; Clarke, S.; Lagopoulos, J.; Hermens, D.F. Is the Theta/Beta EEG Marker for ADHD Inherently Flawed? J. Atten. Disord. 2018, 22, 815-826, 
doi:10.1177/1087054715578270.

41. Clarke, A.R.; Barry, R.J.; Dupuy, F.E.; Heckel, L.D.; McCarthy, R.; Selikowitz, M.;

Johnstone, S.J. Behavioural differences between EEG-defined subgroups of children with Attention-Deficit/Hyperactivity Disorder. Clin. Neurophysiol. 2011, doi:10.1016/j.clinph.2010.12.038.

42. Zhang, D.W.; Li, H.; Wu, Z.; Zhao, Q.; Song, Y.; Liu, L.; Qian, Q.; Wang, Y.; Roodenrys, S.; Johnstone, S.J.; et al. Electroencephalogram Theta/Beta Ratio and Spectral Power Correlates of Executive Functions in Children and Adolescents With AD/HD. J. Atten.

Disord. 2017.

43. Salehinejad, M.A.; Wischnewski, M.; Nejati, V.; Vicario, C.M.; Nitsche, M.A. Transcranial direct current stimulation in attention-deficit hyperactivity disorder: A metaanalysis of neuropsychological deficits. PLoS One 2019, 14, 1-26, doi:10.1371/journal.pone.0215095. 


\section{Chapter 6:}

Summary and Conclusion 


\section{Summary}

This thesis provided evidence supporting the short term safety, tolerability, and acceptability of transcranial direct current stimulation, and has invalidated the theta beta ratio in the context of attention deficit hyperactivity disorder and pediatric neuropsychiatry. This program of research originated from a motivation to utilize tDCS as a treatment option in pediatric neuropsychiatry, specifically, ADHD. Throughout the initial literature review and planning stages in 2015 it became evident that there existed a major discrepancy in the proportion of children compared to adults represented in the tDCS literature $(<5 \%)$ [1]. It was clear that we would have to take a step back and first assess the safety and tolerability of tDCS in children in a preclinical trial before pursuing any clinical treatment trials.

The first step in this program of research was a systematic literature review. This review revealed that there were no serious adverse events reported across 1067 tDCS sessions in 156 children from 12 studies. This provided us with the necessary foundation upon which to design the first randomized double-blind sham-controlled safety trial of tDCS in youth. Having identified the discrepancy of adults and children in the literature, we decided that it would be prudent to examine children and adults in the same safety protocol such that we could facilitate the transferability of typical adult protocols into children. Since the safety of tDCS has already been established in adults, if we could demonstrate that children responded in a similar manner as adults using Bayesian statistics $[2,3]$ then we could be more confident about translating adult tDCS protocols to children. Moreover, by testing multiple electrode sites and amperages we strategically exposed children to a variety of adult protocols therefore maximizing transferability.

We also reported the first account of NNH in the tDCS literature. NNH is a clinically meaningful number used by prescribing physicians to compare and assess risk of a given 
treatment $[4,5]$. The NNH for tDCS is 60 . This means that a clinician should treat 60 patients with a similar tDCS protocol to perhaps have 1 patient reporting a side effect greater than a rating of 0 for a high amperage ( 1 or $2 \mathrm{~mA})$ as compared to a sham or low amperage $(0.5 \mathrm{~mA})$ tDCS protocol. These side effects would most likely be itchiness, tingling, and sleeping problems. Computing NNH for tDCS also allowed us to meaningfully compare risk of harm to pharmaceuticals. An $\mathrm{NNH}$ of 60 is quite large compared to methylphenidate for example. Methylphenidate has an $\mathrm{NNH}$ of 4 for appetite, 7 for wakefulness/insomnia, 9 for stomach aches, 10 for drowsiness, and 11 for dizziness [6] making tDCS around 10 times less harmful than the most commonly prescribed ADHD medication.

Based on the first two studies it became clear that tDCS does appear to be safe and tolerable in children, adolescents, and adults with only mild and very mild side effects. Indeed, more often than not participants reported no side effects at all. However, after conducting Study 2 and 3 it was evident that the safety and tolerability of tDCS ought to be accompanied by a third study of its acceptability. Although tDCS acceptability in youth has proven to be comparable to adults, as indicated by rate of drop outs [1], we realized that the literature was still limited in this regard. Defining acceptability by rate of drop outs is too narrow an approach which fails to allow for the thoughts and feelings of subjects/patients to be considered. From this point of view, it was advantageous for us to dig deeper into tDCS acceptability for children and children with ADHD by interviewing the families from our randomized controlled trial [7].

Until now, perceptions of parents of children exposed to tDCS had not been investigated. Study 3 put the focus on the child and the parent leading to several novel findings important for the translation of tDCS into pediatric neurospsychiatry. Perhaps one of the most important incidental findings is that acceptability, much like consent, is an ongoing process. Acceptability 
began with the participant's willingness to participate in the study, it became concurrent during the actual physical trial itself, and it became prospective in the context of continuing a trial or pursuing the treatment after the trial has ended. This is important because it highlights how tDCS acceptability is part and parcel to the ongoing decision making process of the participants.

Overall, Studies 1-3 provided evidence that tDCS appears to not only be safe and tolerable but also acceptable; so much so that it is even in demand by parents of children with ADHD.

This takes us to Study 5 , the final study of my thesis. This study demonstrated that TBR appears to have no relationship with $\mathrm{ADHD}$ therefore rendering it a moot biomarker. As such, there remains a demand for a reliable biomarker in ADHD. Such a biomarker would enhance prognostics, diagnostics, and neuromodulatory treatment protocols $[8,9]$. The whole field of mental health and neuropsychiatry are moving toward individualized treatment approaches. Previously, some authors have argued that TBR could be a useful EEG biomarker for ADHD making it a potential target to consider for neuromodulation. Indeed multiple large randomized controlled trials attempted to modulate TBR to treat $\mathrm{ADHD}[10,11]$. When these clinical trials on TBR failed, some researchers developed the hypothesis that there may be a TBR threshold $[10,12]$. Their hypothesis was such that children with ADHD are characterized by excess of the TBR threshold and that this comprised a specific phenotype, and this phenotype would benefit from an intervention to decrease TBR. Even though Bussalb et al., [12] was successful in deriving high and low clusters of TBR in ADHD, it was not clear from their analysis that the clusters had anything to do with ADHD. This led us to test the validity of TBR by replicating and extending the clustering / threshold analyses from Bussalb, but this time in healthy children. Our analysis resulted in a very similar TBR threshold (3.725) to Bussalb (3.706) and characterized $34 \%$ of our sample above this threshold, compared to $36 \%$ from Bussalb. Since 
high and low TBR clusters appear to be equally as prevalent in children with ADHD vs. healthy controls, and ADHD the clusters cannot be further explained by ADHD symptomology TBR is not reliable in the context of ADHD. Overall, we submit that this invalidates TBR in ADHD.

\section{Limitations and Future Directions}

Finally, there are some limitations and future directions of my program of research that should be highlighted. Firstly, although the PRISMA guidelines were duly considered in the preparation of our systematic review, the researchers involved in the literature review were not blinded. It is possible that this could have led to some selection bias, however the authors are confident our selection method was robust and in line with our inclusion and exclusion criteria. Secondly, our RCT was limited to evidence for the short term safety and tolerability and future research will have to elucidate long term safety and tolerability of tDCS in order to better align with real world tDCS dosages for ADHD. Thirdly, our acceptability study may have had an implicit bias since the participants being interviewed were already somewhat accepting of tDCS based on their experience in the RCT. That being said, investigating the opinions of families who will actually seek benefit from tDCS is advantageous for the overall ecological validity and clinical translation of tDCS into pediatric neuropsychiatry.

In general, it would have been also interesting if we were able to record EEG from subjects before and after our randomized controlled trial, however the complexity and length of the study design made it impractical. Recording EEG would have also interfered with the interpretation of tolerability related to tDCS. Additionally, the sample size of our RCT was technically underpowered, but the power analysis revealed we would need upwards of 6000 subjects. Therefore, it was more meaningful to report Bayesian statistics [2,3] and the $\mathrm{NNH}$ $[4,5]$. Lastly, in our EEG TBR analysis we used standard theta and beta ranges as opposed to 
individualized frequency ranges based on individual alpha frequency such as Busalb [12] and others [13] have recently suggested. Saad et al [13] posits that computing TBR based on fixed frequency bands may be 'inherently flawed'. It is possible that individualized TBR values could provide more accurate representations of each subject's theta and beta oscillations, however the method we used to compute TBR is congruent with previous literature cited by Bussalb $[14,15]$. This was the same literature that Bussalb compared their thresholds to, and therefore our analysis and thresholds should be equally comparable.

An important future direction of my research program is to design a treatment trial in ADHD utilizing tDCS, EEG, and MRI for full individualization and optimization. Since there is yet to be any clear biomarker to target, it would be useful to collect baseline and follow up neuroimaging outcomes from subjects who undergo a treatment trial in order to reverse engineer prediction models from the baseline EEG and MRI of responders and non-responders. Measuring repeated EEGs would also give us an opportunity to measure the stability of TBR within subjects and within clusters over time. We would also like to investigate additional factors that may explain TBR clusters such as other psychological symptoms or comorbidities, potential cognitive deficits, or arousal. We will elucidate these hypotheses by utilizing additional psychiatric information and the odd-ball and go-no-go event-related potential (ERP) paradigm data also available from the Quebec Newborn Twin Study. To that end, the odd-ball and go-no-go ERP paradigms are each objective electrophysiological measures of impulsivity and attentional control. By combining the values from our TBR dataset with the ERP data we will be able to determine if attentional control can explain the differences in high and low TBR clusters. Since we found tDCS is equally prevalent in healthy children as in ADHD children, this may actually be the more appropriate way to interpret the TBR phenomena. 


\section{Conclusion}

This thesis reported on the safety, tolerability, and acceptability of transcranial direct current stimulation and the validity of the theta beta ratio in the context of attention deficit hyperactivity disorder and pediatric neuropsychiatry. Nearly 100 authors from 10 countries across 4 continents share in the consensus that tDCS is safe in paediatrics. Children and adults find tDCS equally tolerable, reporting on average only very mild and transient side effects with no serious adverse events across 120 sessions in a randomized controlled trial. It appears that further transfer of adult protocols into youth applications is warranted. Parents of children with $\mathrm{ADHD}$ are hopeful that tDCS may become a useful short term intensive treatment option in their child and evidence indicates that they are willing to pursue tDCS if it is prescribed by a physician. That being said, it is crucial for future research to determine and properly validate a biomarker for ADHD in order to optimize tDCS treatment efficacy. Given the poor validity of TBR in ADHD, future research should focus on new biomarkers to enhance tDCS protocols.

It is expected that the results from this thesis will facilitate the overall translation of tDCS into clinical practice. It is recommended that clinical trials and future research proposals regarding TBR be reconsidered to make way for investigations into new biomarkers for ADHD. In conclusion, tDCS appears to be safe, tolerable, and acceptable in neurotypical youth and those with $\mathrm{ADHD}$, but $\mathrm{TBR}$ at $\mathrm{Cz}$ is not a viable target for stimulation. 


\section{References}

1. Bikson, M.; Grossman, P.; Thomas, C.; Zannou, A.L.; Jiang, J.; Adnan, T.;

Mourdoukoutas, A.P.; Kronberg, G.; Truong, D.; Boggio, P.; et al. Safety of Transcranial

Direct Current Stimulation: Evidence Based Update 2016. Brain Stimul. 2016, 9, 641661, doi:10.1016/j.brs.2016.06.004.

2. Biel, A.L.; Friedrich, E.V.C. Why You Should Report Bayes Factors in Your Transcranial Brain Stimulation Studies. Front. Psychol. 2018, 9, 1125, doi:10.3389/fpsyg.2018.01125.

3. Dienes, Z.; Mclatchie, N. Four reasons to prefer Bayesian analyses over significance testing. Psychon. Bull. Rev. 2018, 25, 207-218, doi:10.3758/s13423-017-1266-z.

4. Allen, J. Applying Study Results to Patient Care: Relative Risk, Absolute Risk, and Number Needed to Treat. Pharm. Lett. Prescr. 's Lett. 2005.

5. Citrome, L. Number needed to treat: What it is and what it isn't, and why every clinician should know how to calculate it. J. Clin. Psychiatry 2011.

6. Schachter, H.M.; Pham, B.; King, J.; Langford, S.; Moher, D. How efficacious and safe is short-acting methylphenidate for the treatment of attention-deficit disorder in children and adolescents? A meta-analysis. CMAJ 2001, 165, 1475-1488.

7. Buchanan, D.M.; D'Angiulli, A.; Samson, A.; Maisonneuve, A.R.; Robaey, P. Acceptability of transcranial direct current stimulation in children and adolescents with ADHD: The point of view of parents. J. Health Psychol. 2020, 135910532093705, doi:10.1177/1359105320937059.

8. Snyder, S.M.; Quintana, H.; Sexson, S.B.; Knott, P.; Haque, A.F.M.; Reynolds, D.A. 
Blinded, multi-center validation of EEG and rating scales in identifying ADHD within a clinical sample. Psychiatry Res. 2008, 159, 346-358, doi:10.1016/j.psychres.2007.05.006.

9. Kim, M.; Kwak, Y. Bin; Lee, T.Y.; Kwon, J.S. Modulation of Electrophysiology by Transcranial Direct Current Stimulation in Psychiatric Disorders: A Systematic Review. Psychiatry Investig. 2018, 15, 434-444, doi:10.30773/pi.2018.01.10.

10. Arnold, L.E.; Lofthouse, N.; Hersch, S.; Pan, X.; Hurt, E.; Bates, B.; Kassouf, K.; Moone, S.; Grantier, C. EEG Neurofeedback for ADHD: Double-Blind Sham-Controlled Randomized Pilot Feasibility Trial. J. Atten. Disord. 2013, 17, 410-419, doi: $10.1177 / 1087054712446173$.

11. Schönenberg, M.; Wiedemann, E.; Schneidt, A.; Scheeff, J.; Logemann, A.; Keune, P.M.; Hautzinger, M. Neurofeedback, sham neurofeedback, and cognitive-behavioural group therapy in adults with attention-deficit hyperactivity disorder: a triple-blind, randomised, controlled trial. The Lancet Psychiatry 2017, 4, 673-684, doi:10.1016/S22150366(17)30291-2.

12. Bussalb, A.; Collin, S.; Barthélemy, Q.; Ojeda, D.; Bioulac, S.; Blasco-Fontecilla, H.; Brandeis, D.; Purper Ouakil, D.; Ros, T.; Mayaud, L. Is there a cluster of high theta-beta ratio patients in attention deficit hyperactivity disorder? Clin. Neurophysiol. 2019, 130, 1387-1396, doi:10.1016/j.clinph.2019.02.021.

13. Saad, J.F.; Kohn, M.R.; Clarke, S.; Lagopoulos, J.; Hermens, D.F. Is the Theta/Beta EEG Marker for ADHD Inherently Flawed? J. Atten. Disord. 2018, 22, 815-826, doi:10.1177/1087054715578270. 
14. Clarke, A.R.; Barry, R.J.; Dupuy, F.E.; Heckel, L.D.; McCarthy, R.; Selikowitz, M.; Johnstone, S.J. Behavioural differences between EEG-defined subgroups of children with Attention-Deficit/Hyperactivity Disorder. Clin. Neurophysiol. 2011, doi:10.1016/j.clinph.2010.12.038.

15. Zhang, D.W.; Li, H.; Wu, Z.; Zhao, Q.; Song, Y.; Liu, L.; Qian, Q.; Wang, Y.; Roodenrys, S.; Johnstone, S.J.; et al. Electroencephalogram Theta/Beta Ratio and Spectral Power Correlates of Executive Functions in Children and Adolescents With AD/HD. J. Atten. Disord. 2017. 


\section{Appendices}

Appendix A-1: Study 1 supplementary data

Extended table for tDCS exposure for age and population

\begin{tabular}{|c|c|c|c|c|c|c|c|c|}
\hline Study & $\begin{array}{l}\mathrm{N} \\
\text { (total) }\end{array}$ & $\begin{array}{l}\mathrm{N} \\
\text { (active) }\end{array}$ & $\begin{array}{l}\mathrm{N} \\
\text { (sham) }\end{array}$ & $\begin{array}{l}\mathrm{N} \\
(\min )\end{array}$ & $\begin{array}{l}\text { Amperage } \\
(\mathrm{mA})\end{array}$ & $\begin{array}{l}\text { Age } \\
\text { (years) }\end{array}$ & Population & RCT \\
\hline Mattai & 12 & $5(7)$ & $3(2)$ & 20 & 2, sham & $10-17$ & $\cos$ & Yes \\
\hline (2011) [10] & 175 & 125 & 50 & 2500 & & (16.37) & & \\
\hline Faria & 2 & 2 & 2 & 15 & $.5,1$, sham & $7-11$ & CSWS/LKS & No \\
\hline (2012) [11] & 6 & 4 & 2 & 60 & & (9) & & \\
\hline Auvichayapat & 36 & $20(7)$ & $6(3)$ & 20 & 1 & $6-15$ & Epilepsy & Yes \\
\hline (2013) [12] & 36 & 27 & 9 & 540 & & (11.46) & & \\
\hline Andrade & 14 & $10(4)$ & 0 & 10 & 1,2 & $7-12$ & ELD/PDD- & No \\
\hline (2014) [13] & 140 & 140 & & 1400 & & (7.57) & NOS/AS/GD & \\
\hline Gillick & 1 & 1 & 0 & 10 & 0.7 & 10 & Stroke/Hemi- & Yes \\
\hline (2014) [14] & 1 & 1 & & 10 & & & paretic CP & \\
\hline Gillick & 11 & $3(2)$ & $1(5)$ & 10 & 0.7 & $7-18$ & Congenital & Yes \\
\hline (2015) [15] & 11 & 5 & 6 & 50 & & (14) & Hemiparesis & \\
\hline Moliadze & 19 & $8(11)$ & $8(11)$ & 10 & 1 & $11-16$ & Neurotypical & Yes \\
\hline (2015) [16] & 57 & 38 & 19 & 380 & & (13.9) & & \\
\hline Ciechanski & 24 & $11(7)$ & 3(3) & 20 & 1,2, sham & $13-15$ & Neurotypical & Yes \\
\hline (2016) [17] & 72 & 54 & 18 & 1080 & & (14) & & \\
\hline Kirkton & 24 & $8(4)$ & $8(4)$ & 20 & 1 , sham & $6-18$ & Unilateral & Yes \\
\hline (2016) [18] & 240 & 120 & 120 & 2400 & & (11.5) & $\begin{array}{l}\text { perinatal } \\
\text { stroke }\end{array}$ & \\
\hline Meiron & 1 & 1 & 0 & 20 & $0.1-1$ & 2.5 & Epileptic & No \\
\hline (2017) [19] & 10 & 10 & & 200 & & & Encephalopath & \\
\hline & & & & & & & & Yes \\
\hline Gillick & 20 & $4(6)$ & $5(5)$ & 20 & 0.7 , sham & $7-21$ & UCP via & \\
\hline (2018) [20] & 200 & 10 & 10 & 200 & & (12.75) & $\begin{array}{l}\text { Hemispheric } \\
\text { stroke/PVL }\end{array}$ & Yes \\
\hline Costanzo & 26 & $5(8)$ & $6(7)$ & 20 & 1 , sham & $10-17$ & Dyslexia & \\
\hline (2018) [21] & 468 & 234 & 234 & 4680 & & (13.75) & & \\
\hline Gómez & 15 & $10(5)$ & 0 & 20 & 1 & $5-10$ & ASD & Yes \\
\hline (2017) [22] & 300 & 300 & & 6000 & & (7.7) & & \\
\hline Cole & 24 & $9(7)$ & $2(6)$ & 20 & 1 , sham & $12-18$ & Neurotypical & Yes \\
\hline (2018) [23] & 120 & 80 & 40 & 1600 & & $(15.5)$ & & \\
\hline Rich & 8 & $3(5)$ & 0 & 20 & 1.5 & $7-21$ & Perinatal & No \\
\hline (2018) [24] & 80 & 80 & & 1600 & & (13.4) & stroke/UCP & \\
\hline Costanzo & 23 & 1(11) & 0 & 20 & 1 & $10-18$ & Anorexia & No \\
\hline (2018) [25] & 18 & 18 & & 360 & & (14.5) & Nervosa & \\
\hline
\end{tabular}




\begin{tabular}{|c|c|c|c|c|c|c|c|c|}
\hline Nemanich & 20 & $5(5)$ & $4(6)$ & 20 & 0.7 , sham & $7-21$ & UCP via & Yes \\
\hline (2019) [26] & 200 & 100 & 100 & 2000 & & (12.75) & $\begin{array}{l}\text { Hemispheric } \\
\text { stroke/PVL }\end{array}$ & \\
\hline Ciechanski & 1 & $0(1)$ & 0 & 20 & 1.5 & 17 & Stroke & No \\
\hline (2019) [27] & 10 & 1 & & 200 & & & Hemiparesis & \\
\hline Rahimi & 17 & $9(8)$ & $9(8)$ & 20 & 1 , sham & $9-12$ & Dyslexia & No \\
\hline (2019) [28] & 51 & 34 & 17 & 680 & & (10.35) & & \\
\hline \multirow[t]{3}{*}{ Overall } & 298 & $115(98)$ & $57(60)$ & 15560 & 1,2 & $2.5-21$ & & \\
\hline & 2195 & 1381 & 814 & 423.3 & & (12) & & \\
\hline & & & & (h) & & & & \\
\hline
\end{tabular}

Table 2 summarizes the extent of tDCS exposure for age and population. $\mathrm{N}$ (total) is the total number of individual participants on top and the total quantity of tDCS sessions below. N (active) and N (sham) represents the number of males and females (in parentheses) who received active versus sham tDCS and the number below indicates the quantity of tDCS sessions. $\mathrm{N}$ (min) indicates the duration of each single tDCS session on top, with the number below representing the number of total minutes of active tDCS exposure in each study. Amperage indicates the level of current used in the given sessions. Age is identified first as a range on top and below as an average. Population includes children and adolescents aged $6-18$ years $(\mathrm{COS}=$ Childhood Onset Schizophrenia; $\mathrm{CSWS}=$ Continuous Spikes and Waves during Sleep - Rare Epilepsy; LKS=Landau-Kleffner Syndrome; ELD=Expressive Language Disorder; PDDNOS=Pervasive Developmental Disorder Not Otherwise Specified; $\mathrm{AS}=$ Asperger Syndrome; $\mathrm{GP}=\mathrm{Global}$ Dyspraxia; $\mathrm{CP}=$ Cerebral Palsy; $\mathrm{UCP}=$ Unilateral Cerebral Palsy; $\mathrm{PVL}=$ Periventricular leukomalacia). RCT indicates if the experiment was a randomized controlled trial. 
Extended outcome measures for safety, tolerability, and acceptability.

\begin{tabular}{|c|c|c|}
\hline Study & & \\
\hline \multirow{5}{*}{$\begin{array}{l}\text { Mattai } \\
(2011)\end{array}$} & Neuroimaging & EEG, EKG, MRI \\
\hline & $\begin{array}{l}\text { Tasks } \\
\text { performed/ } \\
\text { Questionnaires/ } \\
\text { Assessment } \\
\text { (Safety) } \\
\end{array}$ & $\begin{array}{l}\text { Clinical interview, neurocognitive testing, California Verbal Learning Test and } \\
\text { Wechsler Memory Scale, clinical ratings (Symptom Assessment for Positive } \\
\text { Symptoms [SAPS] and Brief Psychiatric Rating Scale for Children), routine } \\
\text { physical examination, routine blood work, 12-lead electrocardiogram (EKG), and } \\
\text { a 21-channel electroencephalogram (EEG) performed. }\end{array}$ \\
\hline & $\begin{array}{l}\text { Mode of Side- } \\
\text { Effect } \\
\text { Examination } \\
\text { (Safety/Tolerabi } \\
\text { lity) }\end{array}$ & $\begin{array}{l}\text { Side effects questionnaire during and after treatments. Vital signs, including } \\
\text { pulse, blood pressure, temperature, and respirations, were monitored within the } \\
\text { hour before, during, and immediately after the treatment, and once again } \\
\text { approximately } 8 \text { hours later in the afternoon. Additional daily monitoring, } \\
\text { including a clinical assessment of mental status as well a mini mental status } \\
\text { examination (MMSE), were conducted. Clinical ratings and neurocognitive } \\
\text { testing were conducted on a weekly basis by a trained rater who was blinded to } \\
\text { the parameters of the study. Fisher exact test of independence was used to } \\
\text { compare the proportion of side effects between the active and sham treatment } \\
\text { groups during the blind portion of the study (p values are two tailed). }\end{array}$ \\
\hline & $\begin{array}{l}\text { Adverse Events } \\
\text { (Tolerability) }\end{array}$ & $\begin{array}{l}\text { The most frequent side effect during active treatment was tingling }(\mathrm{n}=6,46.1 \%) \\
\text { or itching }(\mathrm{n}=7,53.8 \%) \text { at the electrode sites. Several patients complained of } \\
\text { fatigue }(\mathrm{n}=4,30.7 \%) \text {. However, this could be related to their medication } \\
\text { regimens. Many subjects reported sham treatment to be associated with tingling } \\
(20.0 \%) \text { and itching }(40.0 \%) \text {. Two subjects also complained of fatigue associated } \\
\text { with sham treatment. Fisher-exact-test for independence comparing side effects } \\
\text { between the active treatment and sham treatment groups during the blind phase of } \\
\text { the study did not reveal any significant differences in tingling (P =.596), itching } \\
(\mathrm{P}=.852) \text {, or fatigue }(\mathrm{P}=.560) \text {. There were no clinically significant changes in } \\
\text { mood, arousal, MMSE, or verbal output during either of the stimulation } \\
\text { conditions. In addition, there were no statistically significant differences in } \\
\text { adverse effects in either treatment group over time. Furthermore, neither } \\
\text { treatment group had significant changes in respiration, blood pressure, heart rate, } \\
\text { EEG, EKG, or MRI, during or after tDCS. The incidence of tingling, itching, and } \\
\text { fatigue in those receiving open active treatment was comparable to the other } \\
\text { treatment groups. }\end{array}$ \\
\hline & $\begin{array}{l}\text { Attrition } \\
\text { (Acceptability) }\end{array}$ & $\begin{array}{l}\text { Neither child asked to stop the study, required a change in CNS medications, or } \\
\text { needed medical intervention because of adverse effects of tDCS treatment. }\end{array}$ \\
\hline \multirow{4}{*}{$\begin{array}{l}\text { Faria } \\
(2012)\end{array}$} & Neuroimaging & EEG \\
\hline & $\begin{array}{l}\text { Tasks } \\
\text { performed/ } \\
\text { Questionnaires } \\
\text { /Assessment } \\
\text { (Safety) } \\
\end{array}$ & $\begin{array}{l}\text { Tolerability assessed using } 1 \text { to } 5 \text { scale where } 1 \text { was "I didn't feel anything" and } 5 \\
\text { was "intolerable". EEG recording during sleep. }\end{array}$ \\
\hline & $\begin{array}{l}\text { Mode of Side- } \\
\text { Effect } \\
\text { Examination } \\
\text { (Safety/Tolerabi } \\
\text { lity) }\end{array}$ & $\begin{array}{l}\text { 5-10 minutes of EEG was recorded as baseline before stimulation, and again after } \\
\text { stimulation. No adverse effects and no complex partial seizures or secondarily } \\
\text { generalized seizures occurred before, during, or after the stimulation. }\end{array}$ \\
\hline & $\begin{array}{l}\text { Adverse Events } \\
\text { (Tolerability) }\end{array}$ & $\begin{array}{l}\text { Intolerability was the only side-effect that was examined. After each TDCS } \\
\text { session, the healthy volunteers classified the tolerability of the tDCS protocol in a }\end{array}$ \\
\hline
\end{tabular}




\begin{tabular}{|c|c|c|}
\hline & & $\begin{array}{l}\text { scale of } 1 \text { to } 5 \text {, where } 1 \text { was "I didn't feel anything" and } 5 \text { was "Intolerable". With } \\
1 \mathrm{~mA} \text { tDCS, participants reported DC application as "I didn't feel anything" } \\
\text { (13\%), "I felt something" ( } 40 \%) \text {, and "Tolerable" ( } 33 \%) \text { and none of them } \\
\text { categorized it as "intolerable". When a current of } 0.5 \mathrm{~mA} \text { was used, only one } \\
\text { participant reported this stimulation as "uncomfortable" and none considered this } \\
\text { session "intolerable". The sham tDCS session confirmed what was expectable, } \\
\text { i.e., } 93 \% \text { of the participants did not feel anything; though some still reported a } \\
\text { sensation. In the epileptiform group, both patients tolerated the procedure well } \\
\text { and reported that they felt something trying to wake them up, when the current } \\
\text { was initially ramped up to } 1 \mathrm{~mA} \text {. Nevertheless, most of the times (5 out of } 6 \\
\text { sessions) after a couple of minutes, they fell asleep. No adverse effects and no } \\
\text { complex partial seizures or secondarily generalized seizures occurred before, } \\
\text { during, or after the stimulation. }\end{array}$ \\
\hline & $\begin{array}{l}\text { Attrition } \\
\text { (Acceptability) }\end{array}$ & No participant attrition was reported. \\
\hline \multirow{3}{*}{$\begin{array}{l}\text { Auvichayapat } \\
\text { (2013) }\end{array}$} & Neuroimaging & EEG \\
\hline & $\begin{array}{l}\text { Tasks } \\
\text { performed/ } \\
\text { Questionnaires } \\
\text { /Assessment } \\
\text { (Safety) }\end{array}$ & $\begin{array}{l}\text { Patients' guardians recorded seizure frequency in a diary for } 4 \text { weeks prior to } \\
\text { treatment and for } 4 \text { weeks after treatment. A 4-week pre-treatment monitoring } \\
\text { period with vital sign measures, EEG, and baseline quality of life (QOL) } \\
\text { questionnaire was done. Follow-ups immediately after stimulation, and at 24, } 48 \\
\text { h, and } 4 \text { weeks after tDCS was done (epileptic discharge counts on EEG \& } \\
\text { seizure diary). The QOL questionnaire was also repeated } 4 \text { weeks after } \\
\text { stimulation. } \\
\text { Patients were closely observed by physicians during and post- } \\
\text { treatment. Vital signs and oxygen saturation were monitored for } \\
698 \mathrm{~N} \text {. Auvichayapat et al. / Brain Stimulation } 6 \text { (2013) } 696 \mathrm{e} 700 \\
30 \text { min prior to and during the treatment period, as well as } 30 \text { min after treatment } \\
\text { and } 48 \text { h after the treatment period. Blood pressure (mm Hg) was measured by } \\
\text { automatic sphygmomanom- eter (Ua-767 Plus, UK) in supine position with } \\
\text { pediatric-size cuff wrapped around the left upper arm. Pulse rate was measured by } \\
\text { automatic sphygmomanometer (Ua-767 Plus, UK) in supine position. Body } \\
\text { temperature was measured by an axillary electronic thermometer. Respiratory rate } \\
\text { was measured by visual inspection, by counting chest risings for } 60 \text { s. Pulse } \\
\text { oximeter was placed on right index finger to monitor oxygen saturation } \\
\text { throughout }\end{array}$ \\
\hline & $\begin{array}{l}\text { Mode of Side- } \\
\text { Effect } \\
\text { Examination } \\
\text { (Safety/Tolerabi } \\
\text { lity) }\end{array}$ & $\begin{array}{l}\text { Repeated-measure ANOVA (groups as a between-subjects factor; time as a } \\
\text { within-subjects factor) revealed a significant main effect of group }(\mathrm{F}(1,34)=7.9 \text {; } \\
\mathrm{P}=0.008) \text {, time }(\mathrm{F}(4,34)=15.1 ; \mathrm{P}<0.0001) \text { and interaction between the two } \\
\text { factors }(\mathrm{F}(4,34=13.0 ; \mathrm{P}<0.0001)) \text {. Post-hoc analysis showed that subjects' } \\
\text { epileptiform discharges frequency was not different between the groups before } \\
\text { the treatment, however it significantly decreased to } 45.3 \% \text { of baseline } \\
\text { immediately after tDCS }(\mathrm{P}=0.0002) \text {, and remained lower in treatment group in } \\
\text { both } 24(50.3 \% ; \mathrm{P}=0.0004) \text { and } 48(57.6 \% ; \mathrm{P}=0.0014) \text { hours after the } \\
\text { treatment. Four weeks after treatment ED frequency increased back to } \\
\text { pretreatment levels and there was no difference between tDCS and sham treated } \\
\text { groups. Post-hoc analysis also showed that clinical seizure frequency decreased } \\
4.8 \% \text { in tDCS group }(\mathrm{P}=0.0035) \text { with no difference in sham treated group. }\end{array}$ \\
\hline
\end{tabular}




\begin{tabular}{|c|c|c|}
\hline & $\begin{array}{l}\text { Adverse Events } \\
\text { (Tolerability) }\end{array}$ & $\begin{array}{l}\text { One patient }(2.7 \%) \text { in active group developed a transient erythematous rash with } \\
\text { no pruritus or pain under reference electrode, which resolved within } 2 \text { hours. No } \\
\text { other adverse effects were reported. }\end{array}$ \\
\hline & $\begin{array}{l}\text { Attrition } \\
\text { (Acceptability) }\end{array}$ & No participant attrition was reported. \\
\hline \multirow{5}{*}{$\begin{array}{l}\text { Andrade } \\
\text { (2013) }\end{array}$} & Neuroimaging & EEG \\
\hline & $\begin{array}{l}\text { Tasks } \\
\text { performed/ } \\
\text { Questionnaires } \\
\text { /Assessment } \\
\text { (Safety) }\end{array}$ & $\begin{array}{l}\text { Activities of social interaction and speech, standardized Adverse-Effect- } \\
\text { Assessment (standard questionnaire proposed by Brunoni et al), given to parents } \\
\text { and participants, also questions in an open-ended manner in order to detect any } \\
\text { additional adverse effects or impressions about the technique, Patient Global } \\
\text { Impression of Improvement, Relevant medical record data: All patients } \\
\text { underwent a complete medical examination prior to and after the transcranial } \\
\text { direct current stimulation. Abnormalities in physical examination, along with } \\
\text { relevant patient complaints, were collected retrospectively, through medical } \\
\text { records. }\end{array}$ \\
\hline & $\begin{array}{l}\text { Mode of Side- } \\
\text { Effect } \\
\text { Examination } \\
\text { (Safety/Tolerabi } \\
\text { lity) }\end{array}$ & $\begin{array}{l}\text { The electroencephalogram showed no alterations in the children's brain (\& } \\
\text { cephalic perimeter). Experimenters also asked questions in an open-ended manner } \\
\text { in order to detect any additional adverse effects or impressions about the } \\
\text { technique. The Patient Global Impression of Improvement is a 1-question } \\
\text { subjective scale that evaluates and ordinates the patients' perception of change } \\
\text { after a particular event, translating it into natural numbers. Its values are } 1 \text { (very } \\
\text { much better), } 2 \text { (much better), } 3 \text { (slightly better), } 4 \text { (no change), } 5 \text { (slightly } \\
\text { worse), } 6 \text { (much worse), and } 7 \text { (very much worse). Although its ability to } \\
\text { accurately detect improvement is somewhat limited, it does provide further } \\
\text { information regarding adverse effects and long-term tolerability. The Patient } \\
\text { Global Impression of Improvement was collected prospectively, through a } \\
\text { telephone-based interview, with the parent or adult responsible for the child } \\
\text { reporting their impressions on the child's reaction to the stimulation. }\end{array}$ \\
\hline & $\begin{array}{l}\text { Adverse Events } \\
\text { (Tolerability) }\end{array}$ & $\begin{array}{l}\text { The main adverse effects reported were acute mood changes (present in } 42.9 \% \text { of } \\
\text { the cases) and irritability }(35.7 \%) \text {. Tingling and itching had an incidence of } \\
28.6 \% \text {, mostly in the mild intensity }(21.4 \%) \text {. Headache, burning sensation, } \\
\text { sleepiness and trouble concentrating were reported by } 14.3 \% \text {, the majority of } \\
\text { them being of them mild. Local redness and scalp pain occurred in } 7.1 \% \text { of } \\
\text { participants. There were no reports of after-treatment seizures during the follow- } \\
\text { up of these subjects. }\end{array}$ \\
\hline & $\begin{array}{l}\text { Attrition } \\
\text { (Acceptability) }\end{array}$ & No participant attrition was reported. \\
\hline \multirow{3}{*}{$\begin{array}{l}\text { Gillick } \\
\text { (2014) }\end{array}$} & Neuroimaging & MRI \\
\hline & $\begin{array}{l}\text { Tasks } \\
\text { performed/ } \\
\text { Questionnaires } \\
\text { /Assessment } \\
\text { (Safety) }\end{array}$ & $\begin{array}{l}\text { The pilot study focused on one 10-year-old child with a diagnosis of arterial } \\
\text { perinatal ischemic stroke. The child had a normal perinatal history but presented } \\
\text { in infancy with hemiparesis and was found to have a focal infarction. Left } \\
\text { hemiparesis was first noted at } 4 \text { months of age. At } 10 \text { years, the child had } \\
\text { moderate hemiparetic cerebral palsy with a Manual Ability Classification System } \\
\text { Scale Score. }\end{array}$ \\
\hline & $\begin{array}{l}\text { Mode of Side- } \\
\text { Effect } \\
\text { Examination } \\
\text { (Safety/Tolerabi }\end{array}$ & $\begin{array}{l}\text { Computerized assessments were made based on digital brain models \& MRI } \\
\text { scans. Workflow (using modeling results) and dosage-consideration in previous } \\
\text { clinical trials was incorporated. Prior ad hoc adult montages vs. de novo } \\
\text { optimized montages provided risk benefit analysis. }\end{array}$ \\
\hline
\end{tabular}




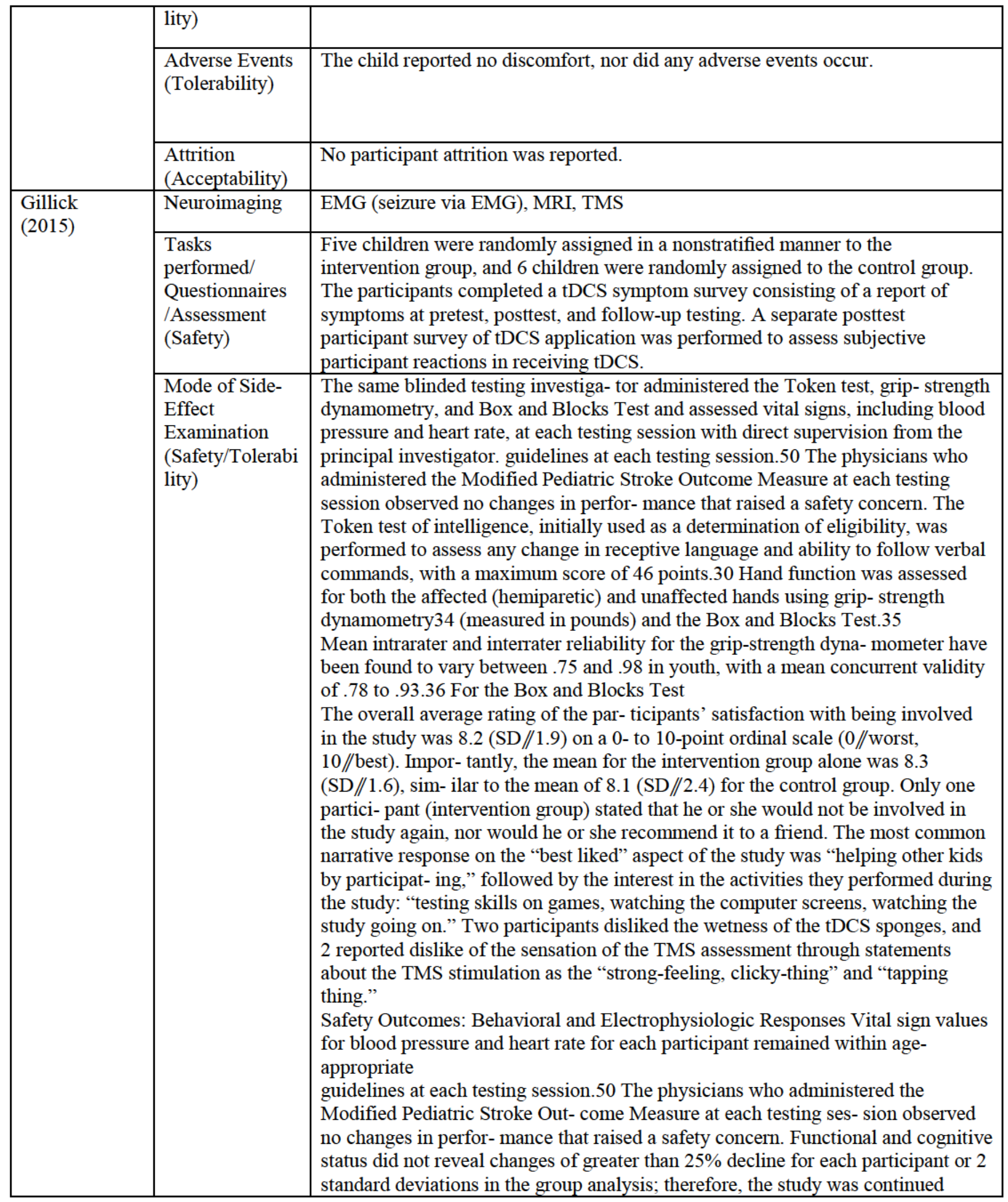




\begin{tabular}{|c|c|c|}
\hline & & $\begin{array}{l}\text { without stopping (Tab. 3, Fig. 2). Of the } 11 \text { children who had an MEP with TMS } \\
\text { assessment, } 10 \text { had resting motor thresholds, and } 1 \text { had an active motor threshold } \\
\text { wherein a maintained active contraction elic- ited an MEP, while at rest an MEP } \\
\text { was not elicited ( }\end{array}$ \\
\hline & $\begin{array}{l}\text { Adverse Events } \\
\text { (Tolerability) }\end{array}$ & $\begin{array}{l}\text { No seizures or other serious adverse events occurred. Minor adverse events } \\
\text { reported were: sensations of itchiness ( } \mathrm{n}=1 \text {, intervention group) and burning ( } \mathrm{n}=1 \text {, } \\
\text { control group) under the area of the electrodes, which stopped } \\
\text { immediately after cessation of the tDCS intervention. Reports of sleepiness ( } \mathrm{n}=3 \text {, } \\
\text { control group; } \mathrm{n}=1 \text {, intervention group) and difficulty concentrating ( } \mathrm{n}=1 \text {, } \\
\text { intervention group; } \mathrm{n}=1 \text {, control group) resolved within } 24 \text { hours. }\end{array}$ \\
\hline & $\begin{array}{l}\text { Attrition } \\
\text { (Acceptability) }\end{array}$ & $\begin{array}{l}2 \text { participants dropped out due to a lack of MEP in the ipsilesional cortex in one } \\
\text { case and discomfort in the other case. ( } 11 \text { children completed the study } \\
\text { procedures). Note that one child (aged } 8 \text { years } 8 \text { months) was excluded prior to } \\
\text { randomization due to inability to elicit a motor evoked } \\
\text { potential(MEP)ineitherhemisphere using TMS. }\end{array}$ \\
\hline \multirow[t]{4}{*}{$\begin{array}{l}\text { Moliadze } \\
(2015)\end{array}$} & Neuroimaging & $\begin{array}{l}\text { EEG, TMS } \\
\text { Nopathologicaloscillations,inparticular,nomarkersofepileptiformactivityafter1mAt } \\
\text { DCSweredetectedinanyofthe EEG analyses } \\
\text { Afteractive1mAtDCstimulationneitherinterictalepileptiformdischargesnorictalEE } \\
\text { G-patternswereobservedinEEG } \\
\text { Allsubjectstoleratedthestimulationwellwithnoseriousadverseevents.Nopatholog- } \\
\text { icaloscillations,nomarkersofepileptiformactivityinEEGweredetectedinanyoftheEE } \\
\text { GtracesafterlmAtDCstimulation. }\end{array}$ \\
\hline & $\begin{array}{l}\text { Tasks } \\
\text { performed/ } \\
\text { Questionnaires } \\
\text { /Assessment } \\
\text { (Safety) }\end{array}$ & $\begin{array}{l}\text { After each experimental session, subjects reported side effects of the stimulation } \\
\text { by a specifically developed questionnaire. Within each type of experimental } \\
\text { session, the measurements were always performed by the same investigator and at } \\
\text { the same time of day for each subject. The experiments were carried out with at } \\
\text { least } 7 \text { days in between. }\end{array}$ \\
\hline & $\begin{array}{l}\text { Mode of Side- } \\
\text { Effect } \\
\text { Examination } \\
\text { (Safety/Tolerabi } \\
\text { lity) }\end{array}$ & \\
\hline & $\begin{array}{l}\text { Adverse Events } \\
\text { (Tolerability) }\end{array}$ & $\begin{array}{l}\text { Anodal TDCS: Mild tingling was reported by } 31.6 \% \text { of subjects during and by } \\
5.3 \% \text { (only one subject) after stimulation. Slight itching sensation under the } \\
\text { electrodes occurred in } 31.6 \% \text { during and in } 5.3 \% \text { (only one subject) after } \\
\text { stimulation. Moderate fatigue was felt by } 15.8 \% \text { of the subjects during tDCS and } \\
21.0 \% \text { after tDCS was reported. } 31.6 \% \text { of subjects felt a slight burning that } \\
\text { quickly receded and } 15.8 \% \text { of them experienced a mild pain sensation under the } \\
\text { electrodes during stimulation. After stimulation, only one subject reported slight } \\
\text { burning. None of the participants reported any pain. None of the participants } \\
\text { reported any visual sensation associated with the switching on and off of the } \\
\text { stimulation; } 16 \% \text { of the volunteers found the stimulation procedure mildly } \\
\text { unpleasant; and none of the participants reported difficulties in concentrating } \\
\text { during and after stimulation. No headaches occurred either during or after } \\
\text { stimulation. Only one subject felt nervous or overexcited during stimulation. } \\
\text { None of them had any changes in visual perception or became hyperactive either } \\
\text { during or after tDCS. } \\
\text { Cathodal TDCS: mild tingling sensation was one of the most common adverse } \\
\text { effects (57.9\% of subjects during and } 5.3 \% \text { (only one subject) after stimulation). }\end{array}$ \\
\hline
\end{tabular}




\begin{tabular}{|c|c|c|}
\hline & & 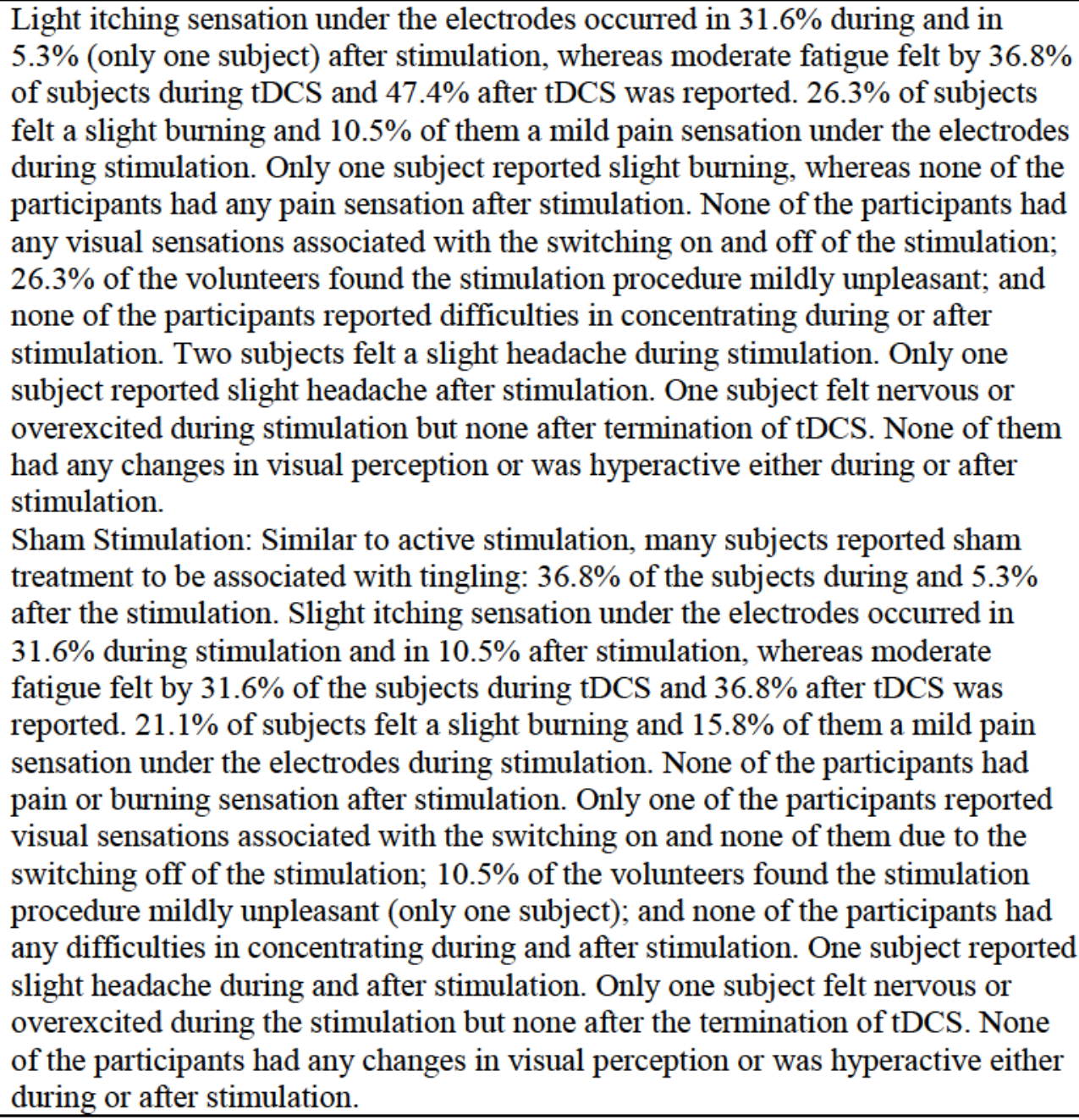 \\
\hline & $\begin{array}{l}\text { Attrition } \\
\text { (Acceptability) }\end{array}$ & None was reported. \\
\hline \multirow{2}{*}{$\begin{array}{l}\text { Gómez } \\
\text { (2017) }\end{array}$} & Neuroimaging & EEG, ERP, EOG \\
\hline & $\begin{array}{l}\text { Tasks } \\
\text { performed/ } \\
\text { Questionnaires/ } \\
\text { Assessment } \\
\text { (Safety) }\end{array}$ & $\begin{array}{l}\text { Tasks performed } \\
\text { During stimulation, patients were either seated and watching TV cartoons of their } \\
\text { choice, or listening to music and playing with small, simple toys. } \\
\text { Event-Related Potential (ERP) assessment was carried out in six children. They } \\
\text { sat in a sound- and light-attenuated room, watching a silent movie, while a } \\
\text { paradigm was delivered through headphones. Said paradigm consisted of } 200 \\
\text { stimuli, } 80 \% \text { frequent ( } 500 \mathrm{~Hz} \text { ) and } 20 \% \text { infrequent target }(1000 \mathrm{~Hz}) \text { tones. In the } \\
\text { passive version of the oddball task, the subject's attention is usually directed away } \\
\text { from the tone sequence toward another task requiring moderate focus. } \\
\text { Considering patients were autistic children, an alternate version was proposed but } \\
\text { met with low collaboration. All stimuli }(50 \mathrm{~ms} ; 5 \mathrm{~ms} \text { rise and fall time) were } \\
\text { presented binaurally with an inter-stimulus interval of } 1300 \text { ms. To record the } \\
\text { auditory P300 ERPs, the stimuli were } 0.1 \mathrm{~ms} \text { alternating clicks. } \\
\text { Functional brain connectivity and autistic behaviour were examined during } \\
\text { treatment. }\end{array}$ \\
\hline
\end{tabular}




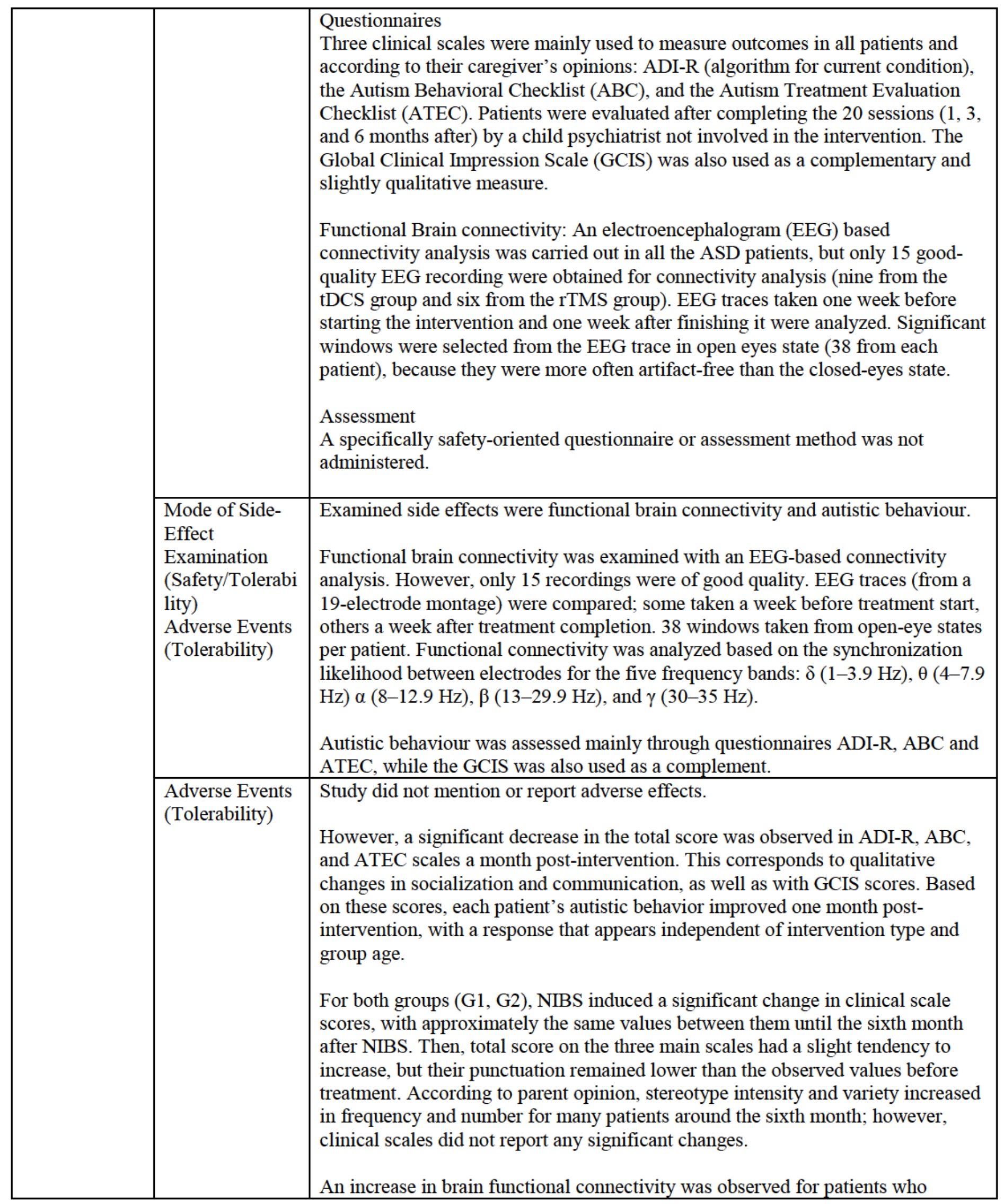




\begin{tabular}{|c|c|c|}
\hline & & $\begin{array}{l}\text { received tDCS or rTMS, especially for higher frequencies. } \alpha, \beta \text {, and } \gamma \text { bands } \\
\text { showed the greatest increase, with a large increment in the number of } 10 / 20 \\
\text { system points functionally related. Effect analysis revealed that the group } \\
\text { receiving rTMS accounted for the most significant changes when comparing } \\
\text { initial and final values. } \\
\text { ERP recordings were only possible in six patients during initial evaluation; thus, } \\
\text { after the intervention, only activity in these same patients was recorded. All had } \\
\text { had a normal auditory brainstem response according to the normative laboratory } \\
\text { data, but one had no ERP response. } \\
\text { The P300 component showed a scalp distribution in the frontal and central } \\
\text { regions, maximal in Fz. There was a statistically significant shortening of P300 } \\
\text { latency after treatment. P300 amplitude lacked significant differences after } \\
\text { treatment, though the average group value became higher. }\end{array}$ \\
\hline & $\begin{array}{l}\text { Attrition } \\
\text { (Acceptability) }\end{array}$ & None was reported. \\
\hline $\begin{array}{l}\text { Meiron } \\
(2017)\end{array}$ & Neuroimaging & $\begin{array}{l}\text { EEG } \\
\text { Frequency of clinical seizures was not significantly reduced. However, interictal } \\
\text { sharp wave amplitudes were significantly lower during the post-intervention } \\
\text { period versus baseline. }\end{array}$ \\
\hline & $\begin{array}{l}\text { Tasks } \\
\text { performed/ } \\
\text { Questionnaires/ } \\
\text { Assessment } \\
\text { (Safety) }\end{array}$ & $\begin{array}{l}\text { EEG was acquired on intervention day } 1,3,5,6,8,10 \text { immediately after the HD- } \\
\text { tDCS sessions. Post HD-tDCS was also recorded on days } 3,10,17,24,31 \text { and } 41 \\
\text { post-intervention for assessment. Neurological assessments were performed } \\
\text { throughout the study period. }\end{array}$ \\
\hline & $\begin{array}{l}\text { Mode of Side- } \\
\text { Effect } \\
\text { Examination } \\
\text { (Safety/Tolerabi } \\
\text { lity) }\end{array}$ & $\begin{array}{l}\text { The clinical functional status of the child, vital signs, and biochemistry were all } \\
\text { assessed throughout the study. Pain intensity was also measured at baseline and } \\
\text { following all HD-tDCS intervention using the behavioral pain scale (BPS). }\end{array}$ \\
\hline & $\begin{array}{l}\text { Adverse Events } \\
\text { (Tolerability) }\end{array}$ & $\begin{array}{l}\text { There were no adverse events or side effects during the course of the study. Stable } \\
\text { vital signs, electrolyte levels and blood chemistry showed no serious adverse } \\
\text { events. Neurological assessments indicated development did not change. The } \\
\text { patient's skin under the electrodes was not irritated and no behavioral changes } \\
\text { (facial expression, arm movements, berating patterns) were noted. } \\
\text { Pulse rate during baseline (mean }=107 \mathrm{bpm}, \mathrm{SD}= \pm 17.16 \mathrm{bpm} \text { ) was normal and } \\
\text { remained within normal limits during interven- tion (mean }=103.35 \mathrm{bpm}, \mathrm{SD}= \pm \\
18.78 \mathrm{bpm} \text { ) and post inter- vention (mean }=92.5 \mathrm{bpm}, \mathrm{SD}= \pm 15.47 \mathrm{bpm}) \\
\text { periods. There were no significant fluctuations in respiratory rate }(20-23 \mathrm{breaths} \\
\text { per minute), temperature }\left(35.1-37.2^{\circ} \mathrm{C}\right) \text {, or blood satura- tion }(98 \%-100 \% \text { SpO2) } \\
\text { throughout the entire study period. Accordingly, the intervention was considered } \\
\text { safe and had no negative impact on the patient's vital signs, electrolyte levels or } \\
\text { blood biochemistry. Importantly, no adverse events or side effects were observed } \\
\text { during the study period. The skin under the stimulating electrodes was not } \\
\text { irritated and the patient did not display any changes in behavior associated with }\end{array}$ \\
\hline
\end{tabular}




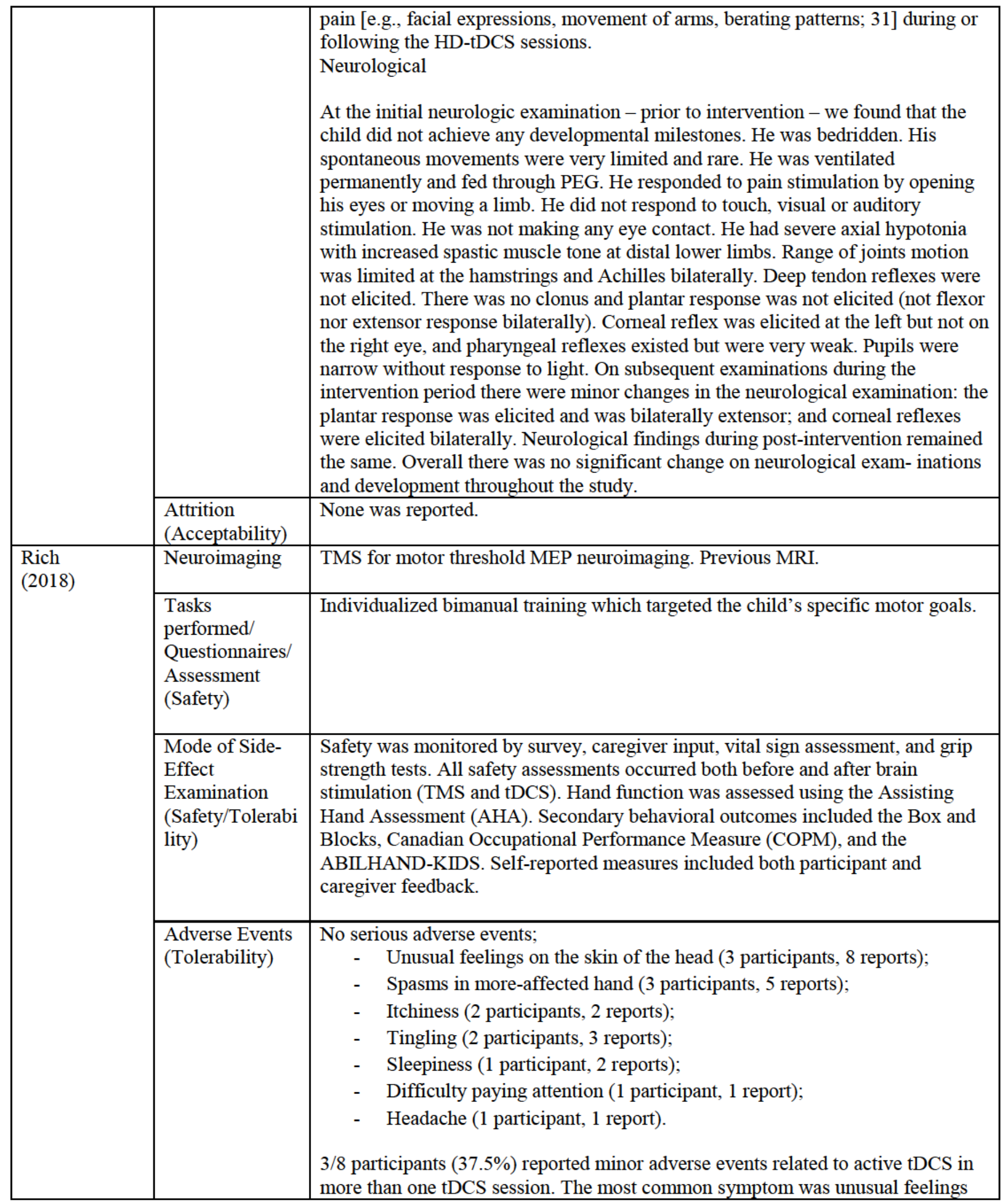




\begin{tabular}{|c|c|c|}
\hline & & $\begin{array}{l}\text { on the skin of the head. } \\
\text { 3.1. Safety Measures.All enrolled participants completedthe study without any } \\
\text { serious adverse events. In this study,37.5\% ( } 3 \text { participants) reported minor } \\
\text { adverse events relatedto active tDCS in more than one tDCS session, with the } \\
\text { mostcommon symptom being unusual feelings on the skin }\end{array}$ \\
\hline & $\begin{array}{l}\text { Attrition } \\
\text { (Acceptability) }\end{array}$ & $\begin{array}{l}\text { One participant was excluded after the preintervention TMS testing due to lack of } \\
\text { bilateral or contralateral circuitry. }\end{array}$ \\
\hline \multirow[t]{5}{*}{$\begin{array}{l}\text { Nemanich } \\
\text { (2019) }\end{array}$} & Neuroimaging & $\begin{array}{l}\text { TMS for motor threshold MEP neuroimaging. Previous MRI. } \\
\text { 4.3. Neurophysiologic Influences.The effects of tDCS can beevaluated through } \\
\text { changes in cortical excitability usingsingle-pulse TMS [21]. }\end{array}$ \\
\hline & $\begin{array}{l}\text { Tasks } \\
\text { performed/ } \\
\text { Questionnaires/ } \\
\text { Assessment } \\
\text { (Safety) }\end{array}$ & $\begin{array}{l}\text { Individualized activities to target child's goal (fine motor activities, leisure } \\
\text { activities, activities of daily living skills etc), with the ultimate goal of improving } \\
\text { unimanual function. Hand function was assessed using the Assisting Hand } \\
\text { Assessment (AHA). Corticospinal excitability of both hemispheres was assessed } \\
\text { by single-pulse TMS. }\end{array}$ \\
\hline & $\begin{array}{l}\text { Mode of Side- } \\
\text { Effect } \\
\text { Examination } \\
\text { (Safety/Tolerabi } \\
\text { lity) }\end{array}$ & $\begin{array}{l}\text { Safety and tolerability were monitored and documented with a participant report } \\
\text { which contained a checklist of symptoms, for both TMS and tDCS interventions. } \\
\text { Adverse events reported by the participant were reviewed by } \\
\text { investigators/caregivers to determine relation to study. Evaluation of the motor } \\
\text { hotspot occurred during the pre-test. Neurophysiological changes were assessed } \\
\text { using single-pulse TMS (including motor threshold, MEP amplitude, cortical } \\
\text { motor mapping, and cortical silent period). TMS testing occurred within } 1 \text { week } \\
\text { prior to intervention and within one week after completion of the } \\
\text { tDCS + bimanual intervention. }\end{array}$ \\
\hline & $\begin{array}{l}\text { Adverse Events } \\
\text { (Tolerability) }\end{array}$ & $\begin{array}{l}\text { No serious adverse events were reported. Transient minor adverse events may } \\
\text { have occurred but were not explicitly reported. }\end{array}$ \\
\hline & $\begin{array}{l}\text { Attrition } \\
\text { (Acceptability) }\end{array}$ & No participant attrition reported. \\
\hline \multirow{2}{*}{$\begin{array}{l}\text { Rahimi } \\
(2019)\end{array}$} & Neuroimaging & EEG \\
\hline & $\begin{array}{l}\text { Tasks } \\
\text { performed/ } \\
\text { Questionnaires } \\
\text { /Assessment } \\
\text { (Safety) }\end{array}$ & $\begin{array}{l}\text { Patients performed the gap-in-noise (GIN) test and long-latency auditory-evoked } \\
\text { potentials (LLAEP) were recorded. For the GIN, sound was played on a CD } \\
\text { player and connected to the right ear with headphones. The gap durations were } 2- \\
3-4-5-6-8-10-12-15-20 \mathrm{~ms} \text { and the silence epoch between every set was } 6 \mathrm{~s} \text {. } \\
\text { Criteria to measure participant performance include the gap-in-noise threshold } \\
\text { (GINTh, which is the shortest silence gap noticed by the subject at least } 4 / 6 \text { times) } \\
\text { in addition to the percentage of correct responses (GINP), as calculated by the } \\
\text { formula [\# of correct intervals detected - false positives total number of } \\
\text { presentations]. } \\
\text { LLAEP recordings occurred in an acoustic room without electrical interference at } \\
\text { baseline, sham, placebo and after tDCS stimulation in } 2 \text { stimulation polarities. To } \\
\text { minimize muscle artifacts during recording, children watched cartoons while } \\
\text { sitting in a chair. Their skin was cleaned with } 10-20 \text { cleansing gel and electrode } \\
\text { impedance/interelectrode impedance were kept below } 5 \mathrm{k} \Omega \text { and } 2 \mathrm{k} \Omega \text { respectively; } \\
\text { the } 10-20 \text { International system was also used for electrode placement. Responses }\end{array}$ \\
\hline
\end{tabular}




\begin{tabular}{|l|l|l|}
\hline & $\begin{array}{l}\text { were measured with Ag-AgCl electrodes on Fz, Cz and Pz, with reference } \\
\text { electrodes on both mastoids (A1/A2) and a ground electrode on Fpz. A 45mV } \\
\text { cutoff was used to reject artifacts in cortical recordings and speech stimuli were } \\
\text { transferred via headphones. }\end{array}$ \\
\hline $\begin{array}{l}\text { Effect } \\
\text { Examination } \\
\text { (Safety/Tolerabi } \\
\text { lity) }\end{array}$ & $\begin{array}{l}\text { None } \\
\text { (Tolerability) }\end{array}$ & $\begin{array}{l}\text { Adverse events were not examined. } \\
\text { As for GINTh and GINP, no difference was found between baseline and sham } \\
\text { conditions. However, GINTh was significantly lower in stimulation conditions } \\
\text { than in baseline or sham conditions (p<0.001), but there was no meaningful } \\
\text { difference between stimulation types (p=0.24). GINP ended significantly higher } \\
\text { for left anode/right cathode stimulation conditions than all others (p<0.001). }\end{array}$ \\
\hline $\begin{array}{l}\text { Attrition } \\
\text { Acceptability) }\end{array}$ & $\begin{array}{l}\text { Brain activity of P1, N1 and P2 occurred after speech stimuli in all patients. There } \\
\text { were no differences between baseline and sham conditions for amplitude and } \\
\text { latency. Stimulation conditions (unilateral and bilateral) led to increased } \\
\text { amplitude of P1, N1 and P2 as compared to baseline/sham, with no significant } \\
\text { difference between stimulation conditions except for P2 (p=0.008). P1, N1 and P2 } \\
\text { latency was decreased after stimulation (p<0.001), with no significant difference } \\
\text { between stimulation types. }\end{array}$ \\
\hline No participant attrition reported. \\
\hline
\end{tabular}

Appendix A) illustrates the extensive criteria that have been used to investigate risks and side effects associated with tDCS in youth. This includes neuroimaging such as: electroencephalography (EEG), magnetic resonance imaging (MRI), transcranial magnetic stimulation (TMS), event related potentials (ERP), electrocardiogram (EKG), electromyography (EMG), and electrooculography (EOG). Side effects were also evaluated using several different measures such as: California Verbal Learning Scale, Wechsler Memory Scale, Brief Psychiatric rating, vital signs, blood work, neurocognitive testing, seizure frequency, quality of life, global clinical impression scale, behavioural pain scale, and other structured symptom surveys. Moreover, many of the aforementioned measurements were collected at multiple time points before and after tDCS. 
Appendix B-1: Study 2 supplementary data

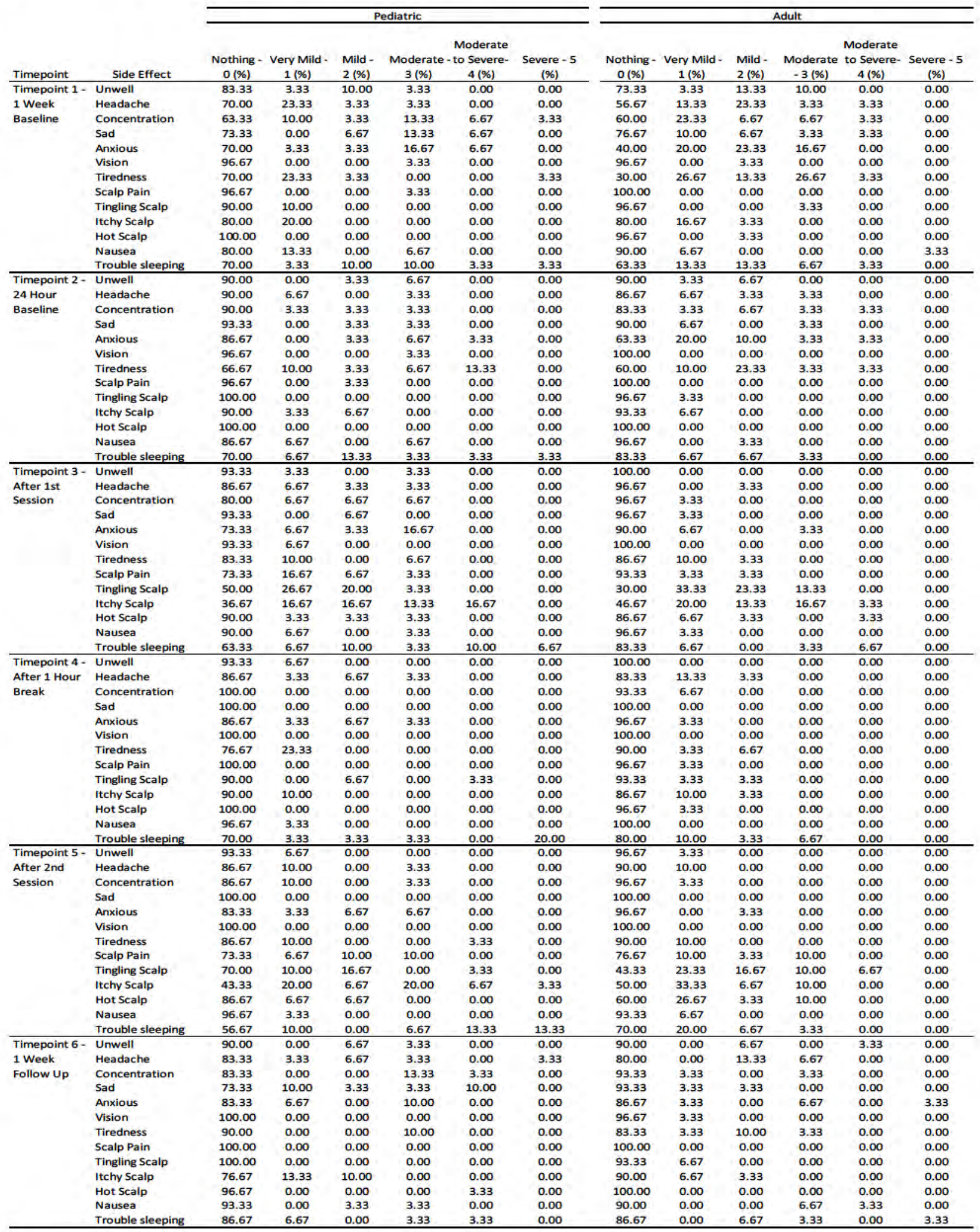




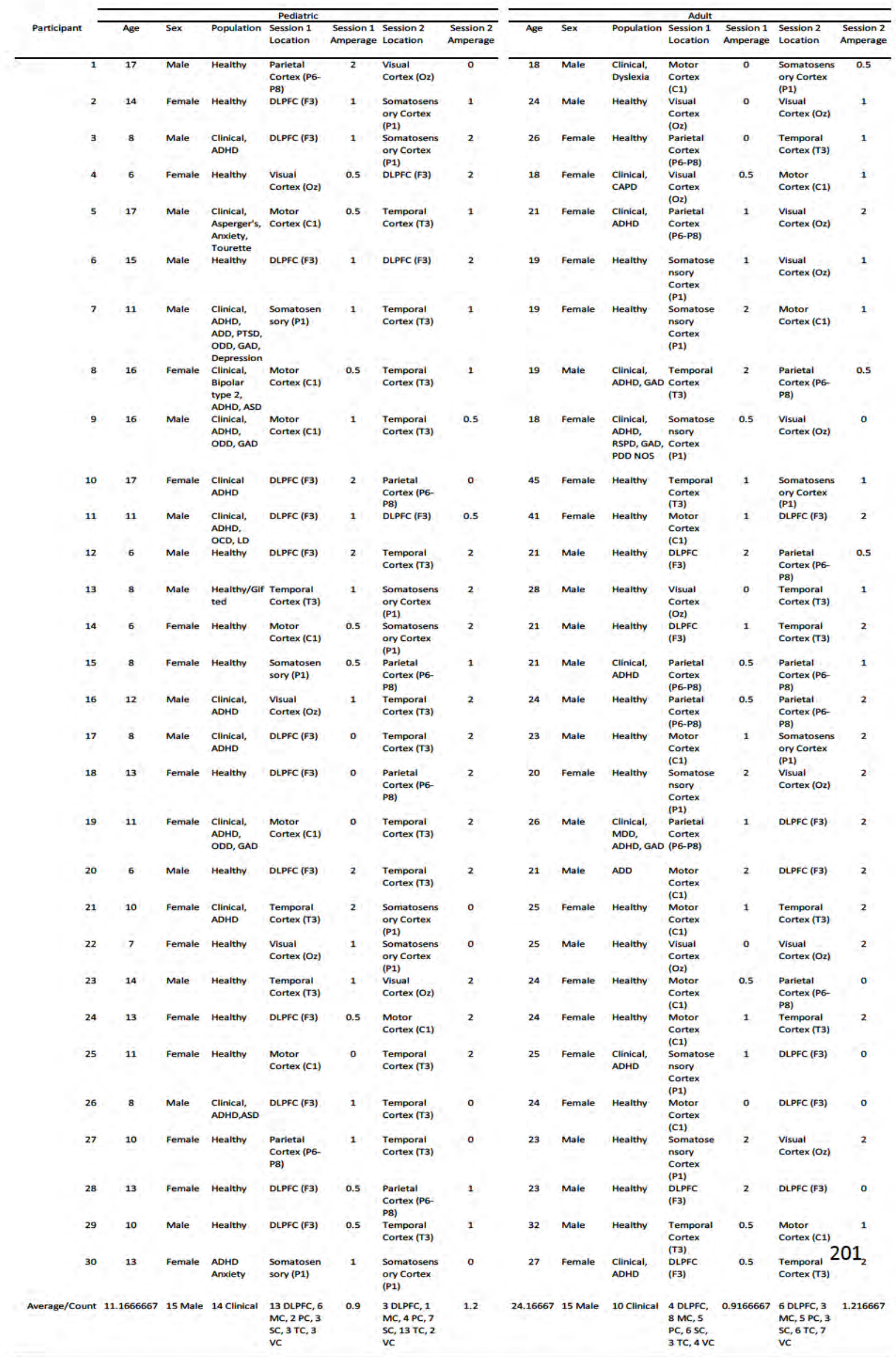


Appendix B-2: Study 2 data collection forms

\begin{tabular}{|l|l|}
\hline Protocol \#: & \\
\hline $\begin{array}{l}\text { Consent Version } \\
\text { Date: }\end{array}$ & \\
\hline Assent Version Date: & \\
\hline Participant ID \#: & \\
\hline
\end{tabular}

This checklist is to serve as record evidence of the informed consent process as per CHEO RI N2 SOP 00807 on Informed Consent Process.

This checklist does not replace the content to be discussed and provided to participants/families in the form of a written informed consent form, as outlined in section 4.8.10 of GCP E6(R2).

\section{Check off to confirm during the informed consent process $\checkmark$}

$\square$ The subject's comprehension of the material reviewed

$\square$ The subject having been given ample opportunity to read the informed consent form and to decide whether or not to participate in the research study

$\square$ Adequate time having been given for all questions about the research study to be answill bed to the satisfaction of the subject

$\square$ Informed consent having been obtained prior to initiating any study-related procedures

$\square$ Copy of signed consent/assent given to participant/family for their records

$\square$ All elements of informed consent process reviewed as per as per CHEO RI N2 SOP 00807 on Informed Consent Process

\section{Any other relevant information:}

\section{Signature:}

Date: 


\section{Meeting with Psychiatrist: Inclusion and Exclusion Criteria}

*All participant's enrolled must meet eligibility criteria based on the inclusion/exclusion criteria detailed in the most current protocol approved by the REB.*

\begin{tabular}{|r|r|}
\hline $\begin{array}{r}\text { Protocol } \\
\text { Title: }\end{array}$ & \\
\hline REB\#: & \\
\hline Participant & \\
ID\#: & \\
\hline
\end{tabular}

\begin{tabular}{|c|c|c|c|}
\hline $\begin{array}{c}\text { Inclusion Criteria } \\
\text { (All must be "yes" for eligibility) }\end{array}$ & Yes & No & Supporting Documentation \\
\hline \multicolumn{4}{|l|}{ 1. Age 6-45 } \\
\hline $\begin{array}{c}\text { Exclusion Criteria } \\
\text { (All must be "no" for eligibility) }\end{array}$ & Yes & No & $\begin{array}{l}\text { Supporting Documentation } \\
\text { (Supporting documentation to } \\
\text { confirm participant eligibility } \\
\text { includes but is not limited to: } \\
\text { laboratory test results, radiology } \\
\text { test results, participant self-report, } \\
\text { and medical records) }\end{array}$ \\
\hline \multicolumn{4}{|l|}{ 1. Pregnant } \\
\hline \multicolumn{4}{|l|}{ 2. Clinical history of seizure/epilepsy } \\
\hline \multicolumn{4}{|l|}{ 3. Clinical history of migraines } \\
\hline 4. Unstable medical condition & & & \\
\hline
\end{tabular}




\begin{tabular}{|c|c|c|}
\hline $\begin{array}{l}\text { 5. Cardiac condition/recent cardiac } \\
\text { surgery }\end{array}$ & & \\
\hline $\begin{array}{l}\text { 6. Neurological conditions such as } \\
\text { brain tumor or electronic implant (eg. } \\
\text { Cochlear implant, pacemaker, metal } \\
\text { braces, metal plates in the head) }\end{array}$ & & \\
\hline $\begin{array}{l}\text { Did participant meet all the } \\
\text { inclusion criteria and did not meet } \\
\text { any exclusion criteria? }\end{array}$ & — $\underset{\text { Yes, participant can be }}{\text { enrolled }}$ & $\begin{array}{l}\text { No, and participant is a } \\
\text { screen failure } \\
\text { enrolled with REB and sponsor } \\
\text { approval* }\end{array}$ \\
\hline \multicolumn{3}{|c|}{ *Please explain if any issues, exceptions, or waivers occur: } \\
\hline
\end{tabular}

Investigator's Signature:

Date:

*Eligibility can only be determined by a licensed physician. Usually this person is the trial investigator or a designated sub-investigator delegated this responsibility on the delegation log.* 


\section{Concomitant Medication Log}

\section{CONCOMITANT MEDICATIONS SOURCE DOCUMENT}

REB\#:

Study Name: Transcranial Direct

Participant ID\#:

Current Stimulation: A safety study in

children and adolescents

\section{Dose Units}

\begin{tabular}{|c|c|c|c|c|c|c|c|}
\hline $\begin{array}{l}\text { BID = } \\
\text { twice } \\
\text { daily } \\
\text { TID = 3 } \\
\text { times/day } \\
\text { QID = 4 } \\
\text { times/day } \\
\text { q2h = } \\
\text { every } 2 \\
\text { hours } \\
\text { q4h = } \\
\text { every } 4 \\
\text { hours }\end{array}$ & 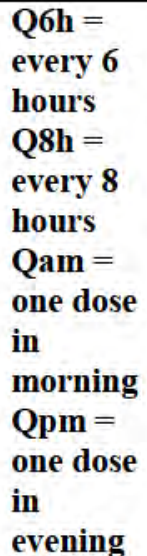 & $\begin{array}{l}\mathrm{QD}= \\
\text { once daily } \\
\text { HS = at } \\
\text { bedtime } \\
\text { PRN = as } \\
\text { needed } \\
\text { OTH = } \\
\text { other } \\
\text { UNK = } \\
\text { unknown }\end{array}$ & $\begin{array}{l}\text { IM - } \\
\text { intramuscular } \\
\text { IN - } \\
\text { intranasal } \\
\text { INH - inhaled } \\
\text { IT - } \\
\text { intrathecally } \\
\text { IV - } \\
\text { intravenous }\end{array}$ & $\begin{array}{l}\text { PO - oral } \\
\text { SC - } \\
\text { subcutaneous } \\
\text { TOP - topical } \\
\text { OTIC - by ear } \\
\text { OTH - other, } \\
\text { specify }\end{array}$ & $\begin{array}{l}\text { g = gram } \\
\text { gtt = drop } \\
\text { mcg = } \\
\text { microgram } \\
\text { mcL = } \\
\text { microliter }\end{array}$ & $\begin{array}{l}\text { mg = } \\
\text { milligram } \\
\text { mL = } \\
\text { milliliter } \\
\text { oz }=\text { ounce } \\
\text { SPY = } \\
\text { spray } \\
\text { supp = } \\
\text { suppository }\end{array}$ & $\begin{array}{l}\text { TBSP }=\text { tablespoon } \\
\text { tsp }=\text { teaspoon } \\
\text { OTH }=\text { other, specify } \\
\text { UNK = unknown }\end{array}$ \\
\hline
\end{tabular}

COMPLETE AT INITIATION OF THERAPY

\begin{tabular}{|c|c|c|c|c|c|c|c|c|}
\hline $\begin{array}{l}\text { MEDICATION / } \\
\text { NON- } \\
\text { MEDICATION } \\
\text { THERAPY }\end{array}$ & $\begin{array}{l}\text { INDICATION } \\
\text { (If given for an } \\
\mathrm{AE} \text {, enter the } \\
\text { exact term } \\
\text { detailed on the } \\
\text { AE form) }\end{array}$ & $\begin{array}{l}\text { BASELINE } \\
\text { MED? } \\
1 \text { - Yes } \\
2 \text { - No }\end{array}$ & $\begin{array}{l}\text { DOSE } \\
\text { (indicate } \\
\text { units) }\end{array}$ & FREQUENCY & ROUTE & $\begin{array}{l}\text { START } \\
\text { DATE } \\
(y y y y- \\
m m m- \\
d d)\end{array}$ & $\begin{array}{l}\text { REPORTER } \\
\text { INITIALS }\end{array}$ & $\begin{array}{r}\text { REPOF } \\
\text { DA] } \\
(y y y y-m)\end{array}$ \\
\hline 1. & & & & & & & & \\
\hline 2. & & & & & & & & \\
\hline 3. & & & & & & & & \\
\hline 4. & & & & & & & & \\
\hline 5. & & & & & & & & \\
\hline 6. & & & & & & & & \\
\hline 7. & & & & & & & & \\
\hline
\end{tabular}


Participant and Parent Side Effect Questionnaires for each time point

\begin{tabular}{|c|c|c|c|c|c|c|c|c|}
\hline $\begin{array}{l}\text { Overall, in the week, did your child } \\
\text { appear to be, or complain of any of } \\
\text { the following? (And if yes, to what } \\
\text { degree and how often?) }\end{array}$ & $\begin{array}{c}0 \\
\text { none/ } \\
\text { good }\end{array}$ & $\begin{array}{c}1 \\
\text { very } \\
\text { mild }\end{array}$ & $\stackrel{2}{2}$ & $\begin{array}{c}3 \\
\text { moder } \\
\text { ate/fai } \\
r\end{array}$ & $\begin{array}{c}4 \\
\text { Severe }\end{array}$ & $\begin{array}{c}5 \\
\text { very } \\
\text { severe/ } \\
\text { poor }\end{array}$ & $\begin{array}{c}\mathrm{N} \\
\text { days/ } \\
\text { week }\end{array}$ & $\begin{array}{c}\mathbf{N} \\
\text { hours/ } \\
\text { day }\end{array}$ \\
\hline \multicolumn{9}{|l|}{ Feeling sick or unwell? } \\
\hline \multicolumn{9}{|l|}{ Having headaches? } \\
\hline \multicolumn{9}{|l|}{$\begin{array}{l}\text { Having trouble concentrating at } \\
\text { school or on homework? }\end{array}$} \\
\hline \multicolumn{9}{|l|}{ Being tearful, prone to cry, or sad? } \\
\hline \multicolumn{9}{|l|}{ Being unusually nervous or anxious? } \\
\hline \multicolumn{9}{|l|}{$\begin{array}{l}\text { Having changes in vision? (e.g., } \\
\text { blurry, phosphenes) }\end{array}$} \\
\hline \multicolumn{9}{|l|}{ Being more tired than usual? } \\
\hline \multicolumn{9}{|l|}{ Having pain on their scalp? } \\
\hline \multicolumn{9}{|l|}{ Feeling tingling on their scalp? } \\
\hline \multicolumn{9}{|l|}{ Having an itchy scalp? } \\
\hline \multicolumn{9}{|l|}{$\begin{array}{l}\text { Feeling a burning or hot sensation on } \\
\text { their scalp? }\end{array}$} \\
\hline \multicolumn{9}{|l|}{ Being nauseous? } \\
\hline \multicolumn{9}{|l|}{ Having trouble sleeping? } \\
\hline \multicolumn{9}{|l|}{$\begin{array}{l}\text { Is there anything else you want to tell } \\
\text { us? }\end{array}$} \\
\hline \multicolumn{9}{|l|}{$\begin{array}{l}\text { Will be there any changes to your } \\
\text { child's routine in the last few days? } \\
\text { Was there anything different or out } \\
\text { of the ordinary about the last few } \\
\text { days? }\end{array}$} \\
\hline Additional information: & & & & & & & & \\
\hline
\end{tabular}




\begin{tabular}{|l|l|l|l|l|l|l|l|l|}
\hline $\begin{array}{c}\text { Overall, in the last week, did you } \\
\text { experience any of the following? (If } \\
\text { yes, to what degree and how often?) }\end{array}$ & $\begin{array}{c}0 \\
\text { none/ } \\
\text { good }\end{array}$ & $\begin{array}{c}1 \\
\text { very } \\
\text { mild }\end{array}$ & $\begin{array}{c}2 \\
\text { Mild }\end{array}$ & $\begin{array}{c}3 \\
\text { moder } \\
\text { ate/fai } \\
\text { r }\end{array}$ & $\begin{array}{c}4 \\
\text { Severe }\end{array}$ & $\begin{array}{c}5 \\
\text { very } \\
\text { severe/ } \\
\text { poor }\end{array}$ & $\begin{array}{c}\text { N } \\
\text { days/ } \\
\text { week }\end{array}$ & $\begin{array}{c}\text { N } \\
\text { hours/ } \\
\text { day }\end{array}$ \\
\hline Feeling sick or unwell? & & & & & & & & \\
\hline Having headaches? & & & & & & & & \\
\hline $\begin{array}{l}\text { Having trouble concentrating at } \\
\text { school or on homework? }\end{array}$ & & & & & & & & \\
\hline Being tearful, prone to cry, or sad? & & & & & & & & \\
\hline Being unusually nervous or anxious? & & & & & & & & \\
\hline $\begin{array}{l}\text { Having changes in vision? (e.g., } \\
\text { blurry, phosphenes) }\end{array}$ & & & & & & & & \\
\hline Being more tired than usual? & & & & & & & & \\
\hline Having pain on their scalp? & & & & & & & & \\
\hline Feeling tingling on their scalp? & & & & & & & & \\
\hline Having an itchy scalp? & & & & & & & & \\
\hline $\begin{array}{l}\text { Feeling a burning or hot sensation on } \\
\text { their scalp? }\end{array}$ & & & & & & & & \\
\hline Being nauseous? & & & & & & & & \\
\hline Having trouble sleeping? & & & & & & & & \\
\hline $\begin{array}{l}\text { Is there anything else you want to tell } \\
\text { us? }\end{array}$ & & & & & & & & \\
\hline
\end{tabular}




\begin{tabular}{|l|l|l|l|l|l|l|l|l|}
\hline $\begin{array}{c}\text { Overall, in the last 24 hours, did your } \\
\text { child } \\
\text { appear to be, or complain of any of } \\
\text { the following? (And if yes, to what } \\
\text { degree and how often?) }\end{array}$ & $\begin{array}{c}0 \\
\text { none/ } \\
\text { good }\end{array}$ & $\begin{array}{c}1 \\
\text { very } \\
\text { mild }\end{array}$ & $\begin{array}{c}2 \\
\text { Mild }\end{array}$ & $\begin{array}{c}3 \\
\text { moder } \\
\text { ate/fai } \\
\text { r }\end{array}$ & $\begin{array}{c}4 \\
\text { Severe }\end{array}$ & $\begin{array}{c}5 \\
\text { very } \\
\text { severe/ } \\
\text { poor }\end{array}$ & $\begin{array}{c}\text { N } \\
\text { hours/ } \\
\text { day }\end{array}$ & $\begin{array}{c}\text { Still } \\
\text { presen } \\
\text { t? }\end{array}$ \\
\hline Feeling sick or unwell? & & & & & & & & \\
\hline Having headaches? & & & & & & & & \\
\hline $\begin{array}{l}\text { Having trouble concentrating at } \\
\text { school or on homework? }\end{array}$ & & & & & & & & \\
\hline Being tearful, prone to cry, or sad? & & & & & & & & \\
\hline Being unusually nervous or anxious? & & & & & & & & \\
\hline $\begin{array}{l}\text { Having changes in vision? (e.g., } \\
\text { blurry, phosphenes) }\end{array}$ & & & & & & & & \\
\hline Being more tired than usual? & & & & & & & & \\
\hline Having pain on their scalp? & & & & & & & & \\
\hline Feeling tingling on their scalp? & & & & & & & & \\
\hline Having an itchy scalp? & & & & & & & & \\
\hline $\begin{array}{l}\text { Feeling a burning or hot sensation on } \\
\text { their scalp? }\end{array}$ & & & & & & & & \\
\hline Being nauseous? & & & & & & & & \\
\hline Having trouble sleeping? & & & & & & & \\
\hline $\begin{array}{l}\text { Is there anything else you want to tell } \\
\text { us? }\end{array}$ & & & & & & & \\
\hline
\end{tabular}




\begin{tabular}{|l|l|l|l|l|l|l|l|l|}
\hline $\begin{array}{l}\text { Overall, in the last 24 hours, did you } \\
\text { experience any of the following? (If } \\
\text { yes, to what degree and how often?) }\end{array}$ & $\begin{array}{c}\text { 0 } \\
\text { none/ } \\
\text { good }\end{array}$ & $\begin{array}{c}1 \\
\text { very } \\
\text { mild }\end{array}$ & $\begin{array}{c}2 \\
\text { Mild }\end{array}$ & $\begin{array}{c}3 \\
\text { moder } \\
\text { ate/fai } \\
\text { r }\end{array}$ & $\begin{array}{c}4 \\
\text { Severe }\end{array}$ & $\begin{array}{c}5 \\
\text { very } \\
\text { severe/ } \\
\text { poor }\end{array}$ & $\begin{array}{c}\text { N } \\
\text { hours/ } \\
\text { day }\end{array}$ & $\begin{array}{c}\text { Still } \\
\text { presen } \\
\text { t? }\end{array}$ \\
\hline Feeling sick or unwell? & & & & & & & & \\
\hline Having headaches? & & & & & & & & \\
\hline $\begin{array}{l}\text { Having trouble concentrating at } \\
\text { school or on homework? }\end{array}$ & & & & & & & & \\
\hline Being tearful, prone to cry, or sad? & & & & & & & & \\
\hline Being unusually nervous or anxious? & & & & & & & & \\
\hline $\begin{array}{l}\text { Having changes in vision? (e.g., } \\
\text { blurry, phosphenes) }\end{array}$ & & & & & & & & \\
\hline Being more tired than usual? & & & & & & & & \\
\hline Having pain on their scalp? & & & & & & & & \\
\hline Feeling tingling on their scalp? & & & & & & & & \\
\hline Having an itchy scalp? & & & & & & & & \\
\hline $\begin{array}{l}\text { Feeling a burning or hot sensation on } \\
\text { their scalp? }\end{array}$ & & & & & & & & \\
\hline Being nauseous? & & & & & & & & \\
\hline Having trouble sleeping? & & & & & & & \\
\hline $\begin{array}{l}\text { Is there anything else you want to tell } \\
\text { us? }\end{array}$ & & & & & & & \\
\hline
\end{tabular}




\begin{tabular}{|c|c|c|c|c|c|c|c|c|}
\hline $\begin{array}{l}\text { During the session, did you experience } \\
\text { any of the following? (If yes, to what } \\
\text { degree and at the beginning or over } \\
\text { the whole session?) }\end{array}$ & $\begin{array}{c}0 \\
\text { none/ } \\
\text { good }\end{array}$ & $\begin{array}{c}1 \\
\text { very } \\
\text { mild }\end{array}$ & $\begin{array}{c}2 \\
\text { mild }\end{array}$ & $\begin{array}{c}3 \\
\text { moder } \\
\text { ate/fair }\end{array}$ & $\begin{array}{c}4 \\
\text { severe }\end{array}$ & $\begin{array}{c}5 \\
\text { very } \\
\text { severe/ } \\
\text { poor }\end{array}$ & $\begin{array}{c}\text { Beginn } \\
\text { ing } \\
\text { only }\end{array}$ & $\begin{array}{c}\text { Over } \\
\text { the } \\
\text { whole } \\
\text { session }\end{array}$ \\
\hline \multicolumn{9}{|l|}{ Did you feel sick or unwell? } \\
\hline \multicolumn{9}{|l|}{$\begin{array}{l}\text { Did you feel any pain in your head, } \\
\text { like a headache? }\end{array}$} \\
\hline \multicolumn{9}{|l|}{$\begin{array}{l}\text { Did you have difficulty } \\
\text { concentrating? }\end{array}$} \\
\hline \multicolumn{9}{|l|}{$\begin{array}{l}\text { Did you feel sad, or did you feel like } \\
\text { crying? }\end{array}$} \\
\hline \multicolumn{9}{|l|}{ Did you feel nervous or scared at all? } \\
\hline \multicolumn{9}{|l|}{$\begin{array}{l}\text { Did you notice anything different in } \\
\text { your vision? (For example, did things } \\
\text { look blurry, phosphenes?) }\end{array}$} \\
\hline \multicolumn{9}{|l|}{ Did you feel tired? } \\
\hline \multicolumn{9}{|l|}{$\begin{array}{l}\text { Did you feel any pain under or } \\
\text { around the electrodes? (Researcher } \\
\text { will point to electrode site) }\end{array}$} \\
\hline \multicolumn{9}{|l|}{$\begin{array}{l}\text { Did you feel any tingling at the } \\
\text { electrode site, like needles or pin } \\
\text { pricks? }\end{array}$} \\
\hline \multicolumn{9}{|l|}{$\begin{array}{l}\text { Does your head feel itchy anywhere, } \\
\text { like you have a bug bite? If yes, } \\
\text { where? }\end{array}$} \\
\hline \multicolumn{9}{|l|}{$\begin{array}{l}\text { Does your head feel hot or like it's } \\
\text { burning at all? If yes, where? }\end{array}$} \\
\hline \multicolumn{9}{|l|}{$\begin{array}{l}\text { Did you feel nauseous, like throwing } \\
\text { up? }\end{array}$} \\
\hline \multicolumn{9}{|l|}{ Did you feel more awake? } \\
\hline \multicolumn{9}{|l|}{ Did the headband feel too tight? } \\
\hline \multicolumn{9}{|l|}{$\begin{array}{l}\text { Is there anything else you want to tell } \\
\text { us? }\end{array}$} \\
\hline $\begin{array}{l}\text { (Researcher may note any changes in } \\
\text { the skin at the electrode site): }\end{array}$ & & & & & & & & \\
\hline
\end{tabular}




\begin{tabular}{|c|c|c|c|c|c|c|c|c|}
\hline $\begin{array}{l}\text { Overall, in the last hour, did you } \\
\text { experience any of the following? (If } \\
\text { yes, to what degree for how long?) }\end{array}$ & $\begin{array}{c}0 \\
\text { none/ } \\
\text { good }\end{array}$ & $\begin{array}{c}1 \\
\text { very } \\
\text { mild }\end{array}$ & $\stackrel{2}{2}$ & $\begin{array}{c}3 \\
\text { moder } \\
\text { ate/fair }\end{array}$ & $\begin{array}{c}4 \\
\text { severe }\end{array}$ & $\begin{array}{c}5 \\
\text { very } \\
\text { severe/ } \\
\text { poor }\end{array}$ & $\begin{array}{c}\text { Beginn } \\
\text { ing } \\
\text { only }\end{array}$ & $\begin{array}{l}\text { Over } \\
\text { the } \\
\text { whole } \\
\text { session }\end{array}$ \\
\hline \multicolumn{9}{|l|}{ Did you feel sick or unwell? } \\
\hline \multicolumn{9}{|l|}{$\begin{array}{l}\text { Did you feel any pain in your head, } \\
\text { like a headache? }\end{array}$} \\
\hline \multicolumn{9}{|l|}{$\begin{array}{l}\text { Did you have difficulty } \\
\text { concentrating? }\end{array}$} \\
\hline \multicolumn{9}{|l|}{$\begin{array}{l}\text { Did you feel sad, or did you feel like } \\
\text { crying? }\end{array}$} \\
\hline \multicolumn{9}{|l|}{ Did you feel nervous or scared at all? } \\
\hline \multicolumn{9}{|l|}{$\begin{array}{l}\text { Did you notice anything different in } \\
\text { your vision? (For example, did things } \\
\text { look blurry, phosphenes?) }\end{array}$} \\
\hline \multicolumn{9}{|l|}{ Did you feel tired? } \\
\hline \multicolumn{9}{|l|}{$\begin{array}{l}\text { Did you feel any pain under or } \\
\text { around the electrodes? (Researcher } \\
\text { will point to electrode site) }\end{array}$} \\
\hline \multicolumn{9}{|l|}{$\begin{array}{l}\text { Did you feel any tingling at the } \\
\text { electrode site, like needles or pin } \\
\text { pricks? }\end{array}$} \\
\hline \multicolumn{9}{|l|}{$\begin{array}{l}\text { Does your head feel itchy anywhere, } \\
\text { like you have a bug bite? If yes, } \\
\text { where? }\end{array}$} \\
\hline \multirow{2}{*}{\multicolumn{9}{|c|}{$\begin{array}{l}\text { Does your head feel hot or like it's } \\
\text { burning at all? If yes, where? }\end{array}$}} \\
\hline & & & & & & & & \\
\hline \multicolumn{9}{|l|}{$\begin{array}{l}\text { Did you feel nauseous, like throwing } \\
\text { up? }\end{array}$} \\
\hline \multicolumn{9}{|l|}{ Did you feel more awake? } \\
\hline \multicolumn{9}{|l|}{ Did the headband feel too tight? } \\
\hline \multicolumn{9}{|l|}{$\begin{array}{l}\text { Is there anything else you want to tell } \\
\text { us? }\end{array}$} \\
\hline $\begin{array}{l}\text { (Researcher may note any changes in } \\
\text { the skin at the electrode site): }\end{array}$ & & & & & & & & \\
\hline
\end{tabular}




\begin{tabular}{|c|c|c|c|c|c|c|c|c|}
\hline $\begin{array}{l}\text { Overall, since the session, have you } \\
\text { noticed or has your child complained } \\
\text { of any of the following? (And if yes, } \\
\text { to what degree and how often?) }\end{array}$ & $\begin{array}{c}0 \\
\text { none/ } \\
\text { good }\end{array}$ & $\begin{array}{c}1 \\
\text { very } \\
\text { mild }\end{array}$ & $\begin{array}{c}2 \\
\text { Mild }\end{array}$ & $\begin{array}{c} \\
\text { moder } \\
\text { ate/fai } \\
\mathbf{r}\end{array}$ & $\begin{array}{c}4 \\
\text { Severe }\end{array}$ & $\begin{array}{c}5 \\
\text { very } \\
\text { severe/ } \\
\text { poor }\end{array}$ & $\begin{array}{c}\mathbf{N} \\
\text { days/ } \\
\text { week }\end{array}$ & $\begin{array}{c}\mathbf{N} \\
\text { hours } \\
\text { day }\end{array}$ \\
\hline \multicolumn{9}{|l|}{ Feeling sick or unwell? } \\
\hline \multicolumn{9}{|l|}{ Having headaches? } \\
\hline \multicolumn{9}{|l|}{$\begin{array}{l}\text { Having trouble concentrating at } \\
\text { school or on homework? }\end{array}$} \\
\hline \multicolumn{9}{|l|}{ Being tearful, prone to cry, or sad? } \\
\hline \multicolumn{9}{|l|}{ Being unusually nervous or anxious? } \\
\hline \multicolumn{9}{|l|}{$\begin{array}{l}\text { Having changes in vision } \\
\text { (phosphenes)? }\end{array}$} \\
\hline \multicolumn{9}{|l|}{ Being more tired than usual? } \\
\hline \multicolumn{9}{|l|}{ Having pain on their scalp? } \\
\hline \multicolumn{9}{|l|}{ Feeling tingling on their scalp? } \\
\hline \multicolumn{9}{|l|}{ Having an itchy scalp? } \\
\hline \multicolumn{9}{|l|}{$\begin{array}{l}\text { Feeling a burning or hot sensation on } \\
\text { their scalp? }\end{array}$} \\
\hline \multicolumn{9}{|l|}{ Being nauseous? } \\
\hline \multicolumn{9}{|l|}{ Having trouble sleeping? } \\
\hline \multicolumn{9}{|l|}{$\begin{array}{l}\text { Is there anything else you want to tell } \\
\text { us? }\end{array}$} \\
\hline \multicolumn{9}{|l|}{$\begin{array}{l}\text { Will be there any changes to your } \\
\text { child's routine in the last few days? } \\
\text { Was there anything different or out } \\
\text { of the ordinary about the last few } \\
\text { days? }\end{array}$} \\
\hline Additional information: & & & & & & & & \\
\hline
\end{tabular}




\begin{tabular}{|c|c|c|c|c|c|c|c|c|}
\hline $\begin{array}{l}\text { Overall, since the session, did you } \\
\text { experience any of the following? (If } \\
\text { yes, to what degree and how often?) }\end{array}$ & $\begin{array}{c}0 \\
\text { none/ } \\
\text { good }\end{array}$ & $\begin{array}{c}1 \\
\text { very } \\
\text { mild }\end{array}$ & $\begin{array}{c}2 \\
\text { Mild }\end{array}$ & $\begin{array}{c}3 \\
\text { moder } \\
\text { ate/fai } \\
\mathbf{r}\end{array}$ & $\begin{array}{c}4 \\
\text { Severe }\end{array}$ & $\begin{array}{c}5 \\
\text { very } \\
\text { severe/ } \\
\text { poor }\end{array}$ & $\begin{array}{c}\mathbf{N} \\
\text { days/ } \\
\text { week }\end{array}$ & $\begin{array}{c}\mathbf{N} \\
\text { hours/ } \\
\text { day }\end{array}$ \\
\hline \multicolumn{9}{|l|}{ Feeling sick or unwell? } \\
\hline \multicolumn{9}{|l|}{ Having headaches? } \\
\hline \multicolumn{9}{|l|}{$\begin{array}{l}\text { Having trouble concentrating at } \\
\text { school or on homework? }\end{array}$} \\
\hline \multicolumn{9}{|l|}{ Being tearful, prone to cry, or sad? } \\
\hline \multicolumn{9}{|l|}{ Being unusually nervous or anxious? } \\
\hline \multicolumn{9}{|l|}{$\begin{array}{l}\text { Having changes in vision } \\
\text { (phosphenes)? }\end{array}$} \\
\hline \multicolumn{9}{|l|}{ Being more tired than usual? } \\
\hline \multicolumn{9}{|l|}{ Having pain on their scalp? } \\
\hline \multicolumn{9}{|l|}{ Feeling tingling on their scalp? } \\
\hline \multicolumn{9}{|l|}{ Having an itchy scalp? } \\
\hline \multicolumn{9}{|l|}{$\begin{array}{l}\text { Feeling a burning or hot sensation on } \\
\text { their scalp? }\end{array}$} \\
\hline \multicolumn{9}{|l|}{ Being nauseous? } \\
\hline \multicolumn{9}{|l|}{ Having trouble sleeping? } \\
\hline \multicolumn{9}{|l|}{$\begin{array}{l}\text { Is there anything else you want to tell } \\
\text { us? }\end{array}$} \\
\hline \multirow{2}{*}{\multicolumn{9}{|c|}{$\begin{array}{l}\text { Was anything different in your life in } \\
\text { the last few days (e.g. changes in your } \\
\text { schedule or routine)? If yes, what? } \\
\text { Additional information: }\end{array}$}} \\
\hline & & & & & & & & \\
\hline
\end{tabular}


Researcher Log Session 1

Participant ID:

Date of Session:

Electrode Site:

Level of Current:

Participant Gender:

Participant D.O.B.: $\quad$ Age $=$

Type of Recruitment:

Changes at Electrode Site (if any; describe if applicable):

\begin{tabular}{|l|l|}
\hline Symptom & Description \\
\hline Redness & \\
\hline Dryness & \\
\hline Skin blanches when pressure is applied & \\
\hline Blistering & \\
\hline Child indicates pain at site & \\
\hline
\end{tabular}


Researcher Log Session 2

Participant ID:

Date of Session:

Electrode Site:

Level of Current:

Participant Gender:

Participant D.O.B.: $\quad$ Age $=$

Type of Recruitment:

Changes at Electrode Site (if any; describe if applicable):

\begin{tabular}{|l|l|}
\hline Symptom & Description \\
\hline Redness & \\
\hline Dryness & \\
\hline Skin blanches when pressure is applied & \\
\hline Blistering & \\
\hline Child indicates pain at site & \\
\hline
\end{tabular}




\section{Appendix C-1: Study 3 data collection forms}

\section{Interview Questions}

To be verbalized to participant by interviewer: As part of a study on the experiences of tDCS use in children and adolescents, I would like to ask you about your experiences (and opinions for those who declined participation in the safety and tolerability study). Please describe your experiences with as much detail as possible and to the best of your ability. There are no 'wrong' answers and no judgment will be placed. If a question makes you feel uncomfortable, please let me know and that question will be skipped. I would like to remind you that you are free to quit the study at any point during the interview with no repercussions from your doctors or the researchers. Would you like us to clarify anything before I begin?

1. Could you please describe to me what you thought about transcranial direct current stimulation when you first heard about it, and have you ever heard about it before being asked to participate? How did you react when asked to participate and what was your perception?

2. What factors did you consider when choosing whether or not to participate in the transcranial direct current stimulation sessions? Please describe for me how these factors played into your decision-making process?

3. How did you make your final decision whether or not to participate? What was the biggest influence or factor that lead you to make the decision you made?

4. Could you please describe for me any concerns you may have had regarding the potential side effects of tDCS on your child?

5. Now that you have seen what a tDCS session looks like, could you please describe to me what you think about tDCS now compared to when you first heard about it? How does it compare to what you imagined?

6. What are the main concerns that you would like to be addressed for using tDCS in children-- or what could influence your perception negatively or positively about using tDCS in children?

7. Based on the potential side effects, how acceptable/or not are these risks to you if you will be to seek tDCS as a clinical treatment? How would you evaluate these potential side effects in comparison to the side effects of other treatments like medications?

8. Generally, what would you have to say about the safety of tDCS? If you will be to rate the safety based on what you have seen, how safe would you say it is $(0-10)$ ?

9. Today, for clinical treatments, there are medications, as well as these other approaches such as tDCS. If you will be to weigh the choise of using tDCS against other methods, what would be the factors that influence your decision? (eg. Effectiveness, cost, duration)

10. If tDCS was proven to be a more effective treatment than a given pharmaceutical, would you prefer treating your child with tDCS or with pharmaceuticals? And what would be the factors that influence your decision of using tDCS? Would cost of the tDCS treatment play a role in your decision?

11. If the combination of $\mathrm{tDCS}$ plus pharmaceuticals was proven to be the most effective treatment for a disorder, would you prefer treating your child with a combined approach? What would be the factors that influence your decision? Would the additional cost of tDCS, above the cost of medication, play a role in your decision?

12. Is there anything in particular that you associated transcranial direct current stimulation with when you first heard about it, versus now? What is your overall perspective on tDCS use as a treatment? In children, and in adults? 


\section{Participant Demographic Questionnaire}

Please circle the most accurate response, or fill in the blank below (please leave the response blank if you wish to not answer a question):

1. I am_ years old.

2. I identify my race as

3. My highest level of education is (please list your degree/diploma/certificate of any education beyond high school):

\section{Questions below are for parents only:}

4. What is your profession?

5. Regarding annual income, please circle the most accurate response:

\begin{tabular}{ll}
\hline Unattached individuals & Families of 2 or more \\
\hline$\$ 0$ to $\$ 20,000$ & $\$ 0$ to $\$ 40,000$ \\
$\$ 20,000$ to $\$ 25,000$ & $\$ 40,000$ to $\$ 60,000$ \\
$\$ 25,000$ to $\$ 40,000$ & $\$ 60,000$ to $\$ 90,000$ \\
$\$ 40,000$ to $\$ 55,000$ & $\$ 90,000$ to $\$ 120,000$ \\
$\$ 55,000$ to $\$ 75,000$ & $\$ 120,000$ to $\$ 150,000$ \\
$\$ 75,000$ to $\$ 100,000$ & $\$ 150,000$ to $\$ 200,000$ \\
$\$ 100,000$ and up & $\$ 200,000$ and up \\
\hline
\end{tabular}


Appendix C-2 Theoretical Framework of Acceptability and tDCS

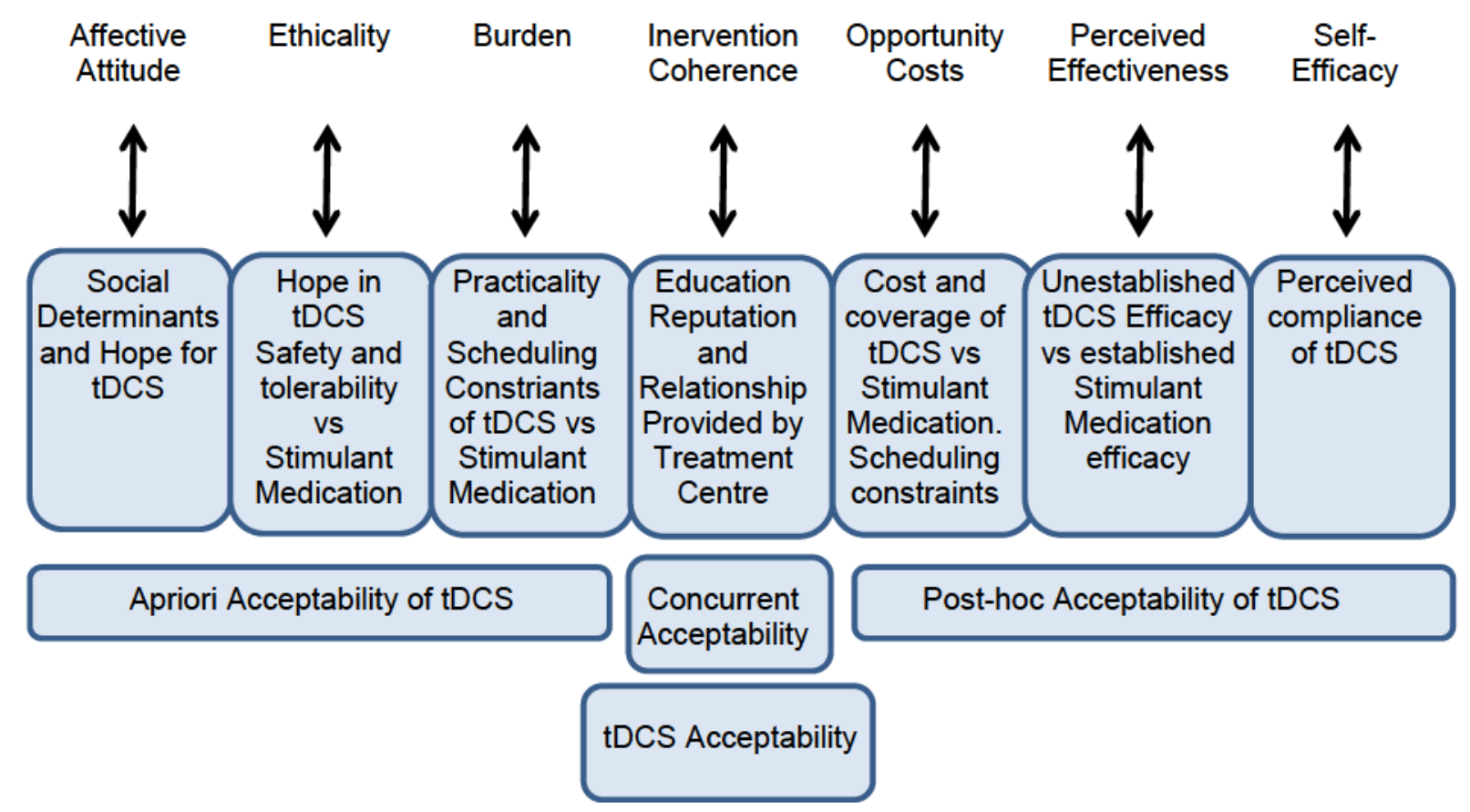


Appendix D-1 Study 4 supplementary data

Boxplots for Zygosity
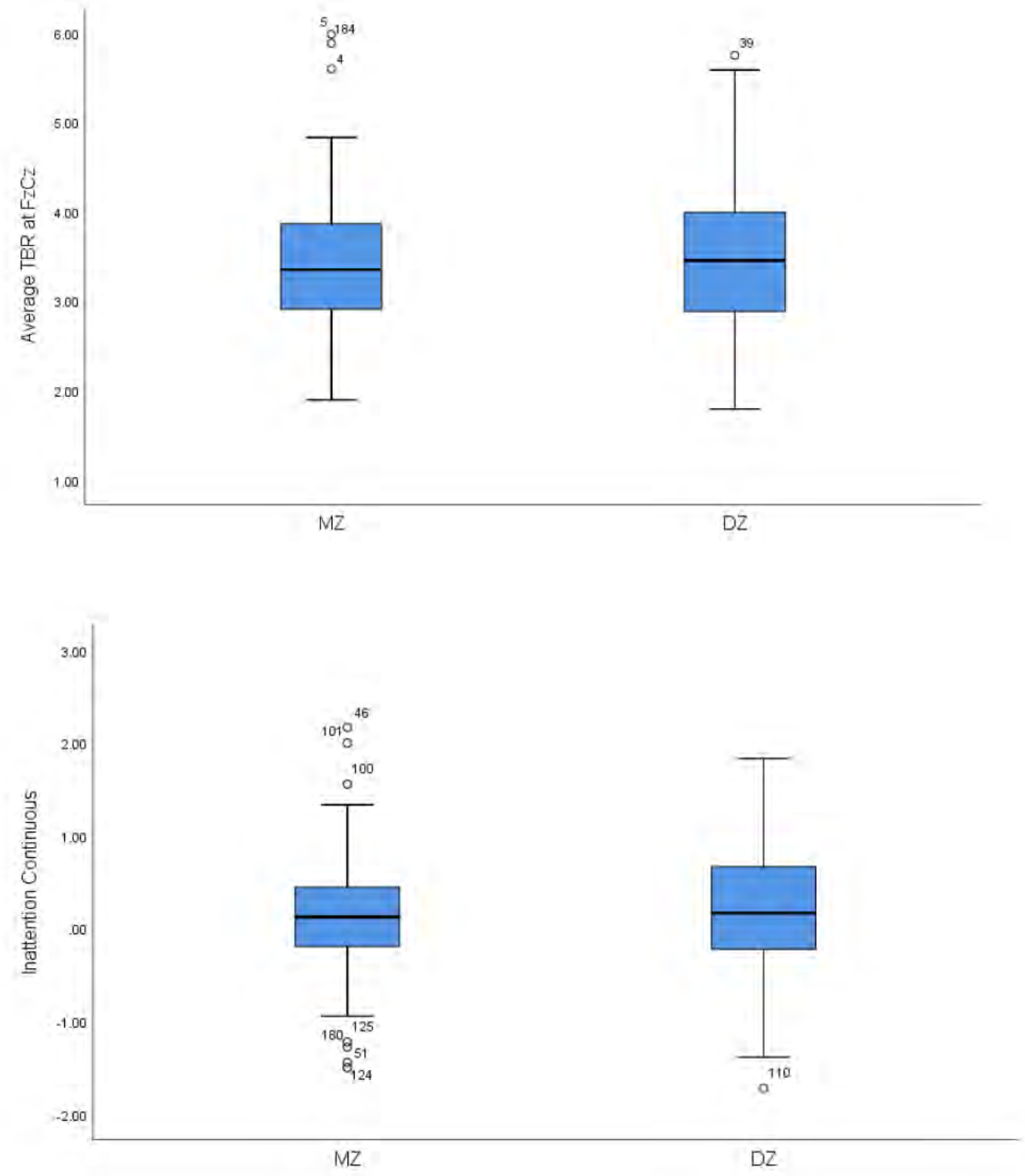

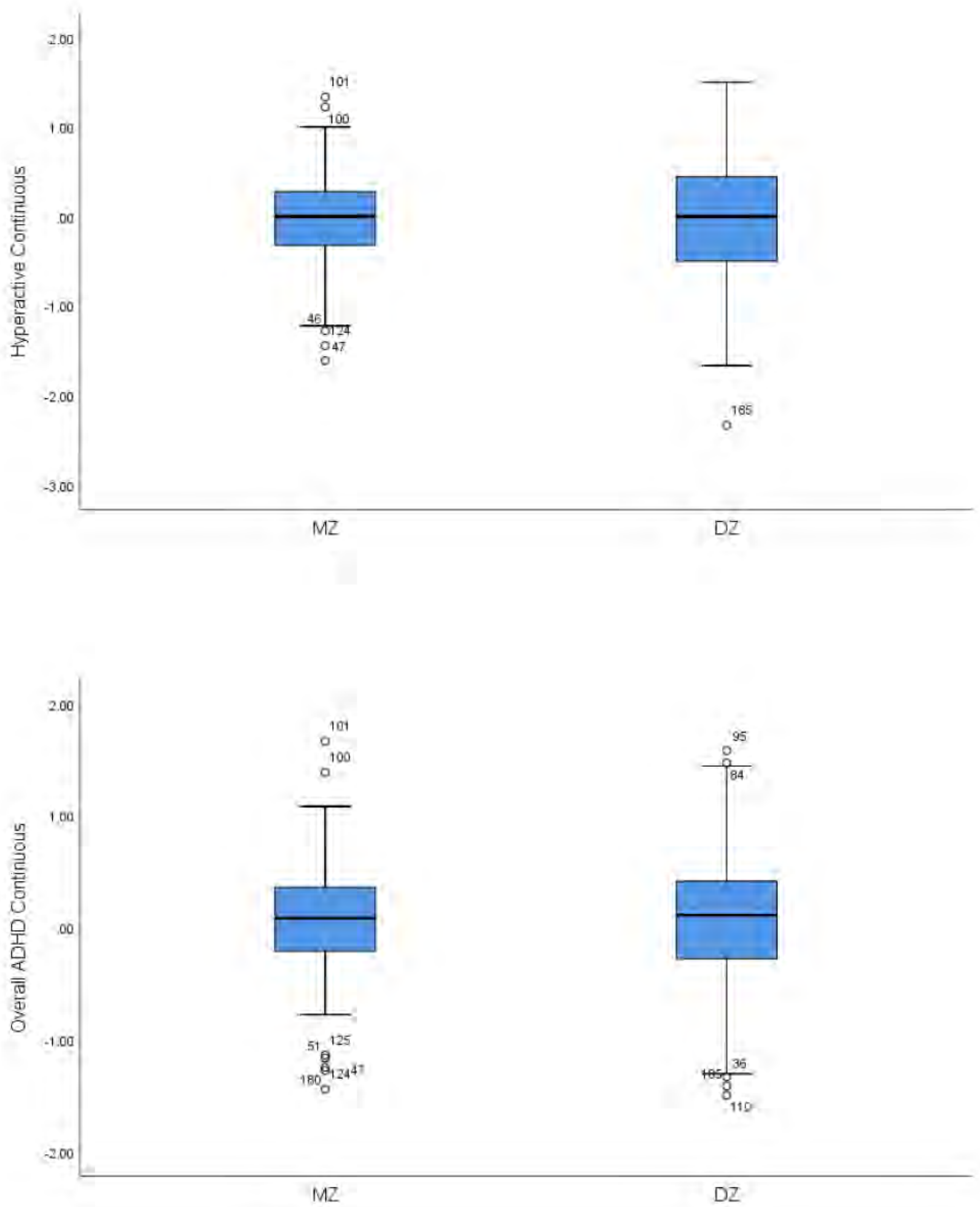
Appendix D-2: Study 4 EEG acquisition set up and testing battery

Subjects

The Quebec Newborn Twin Registry ascertained from all twin births occurring in the Province of Quebec between 1 April, 1995 and 31 December, 1998. Names, addresses, and phone numbers of all mothers of newborn twins were collected every 14 days from the computerized birth records of the Quebec Bureau of Statistics. An elaborate process of recruitment and maintenance of families in the Registry was managed through periodic mail and telephone contacts, newsletters and follow-ups. Since 1 June 1995, all parents living in the Greater Montreal Area were asked to enroll with their twins in the Quebec Newborn Twin Study (QNTS). Half of the sample i.e., 325 pairs of 5-month old twins (corrected for gestational age) have been evaluated in the laboratory and at home starting June 1, 1996, for those born after January 1, 1996. Home visits without laboratory assessment were also conducted on the other half of the sample ( 325 pairs) also aged 5 months. The total sample ( 650 pairs) has been followed longitudinally at home at 18,30,48 and 60 months. The children will be $7-8$ years old at evaluation. Based on previous experience, children of this age are able to complete all of our diagnostic and assessment measures.

Stimulus and task

The Kid-NET psychophysiological battery consists of 8 tasks: (1) Baseline EEG measurement with eyes open; (2) Baseline EEG measurement with eyes close; (3) Oddball task; (4) Hybrid Stop-Go/No-go task; (5) Visual-Spatial Working Memory task (experimental condition); (6) Visual-Spatial Working Memory task (control condition); (7) Visual-Spatial Working Memory task (two-probe condition);

Baseline measurement during eyes open

A fixation point was presented at the light gray background center of a Dell ${ }^{\mathrm{TM}} 1800 \mathrm{FP}$ color monitor for 3 minutess. Subjects were asked to fix on the screen center of the monitor with eyes open.

Baseline measurement during eyes close

A fixation point was presented at the light gray background center of a Dell ${ }^{\mathrm{TM}} 1800 \mathrm{FP}$ color monitor for 3 minutess. Subjects were asked to fix on the screen center of the monitor with eyes close.

Oddball task

Stimuli were line drawings depicting either a moose (Frequent, $75 \%$ ) or a raccoon (Rare, 25\%) presented at the light gray background center of a Dell ${ }^{\mathrm{TM}} 1800 \mathrm{FP}$ color monitor. Each drawing extended approximately $10 \mathrm{~cm}(6.3$ degrees at $90 \mathrm{~cm})$ and was presented against a white 
background. A single block of 160 stimuli was delivered. Each stimulus was presented for 100 $\mathrm{ms}$ and the inter-trial interval varied between 2200 and $2800 \mathrm{~ms}$. All subjects received the same pseudorandom stimulus presentation order, which was restricted so that two rare stimuli never occurred in succession. Subjects were asked to keep their lower arms rested on a table fitted to the chair and each hand rested over one of the two large $(5 \mathrm{~cm})$ red response buttons attached to the table. They were instructed to press one of the two buttons after the onset of the stimulus, using the left hand for Frequent stimulus (i.e. moose) and the right hand for Rare (i.e. raccoon) stimuli. Responses were classified as correct if they occurred within 200-1800 ms after stimulus onset. The task lasted approximately $7 \mathrm{~min}$.

\section{Hybrid-Stop task}

Subjects were presented with a series of visual stimuli at the light gray background center of a Dell $^{\mathrm{TM}}$ 1800FP color monitor. Blue and black arrows $(4 \times 4.5 \mathrm{~cm})$ were used as stimuli for the task. For the Go or No-Go trials, black or blue arrows pointing either to the left or right direction were presented following a blank flash $(500 \mathrm{~ms})$. The colors of arrows determined the response condition as either Go or No-Go trials. For the stop trial, a blank flash $(500 \mathrm{~ms})$ and a Go arrow were presented at first. After a short delay, a Stop Signal (No-Go arrow) was presented. Subject must withhold their response whenever a stop signal was presented. If the subject could successfully withhold their responding, the program would increase the delay (stimulus onset asynchrony, SOA) by $50 \mathrm{~ms}$. In contrast, if the subject was successfully inhibiting his(her) response to stop trials, the program would decrease the SAO by $50 \mathrm{~ms}$ for the next stop trial, making the next stop trial more difficult. Subjects were instructed to use the hand in the direction of the arrow and to squeeze a strength gauge as quickly as possible in responding to the arrow for the Go trials, but withhold the action for the No-Go and the Stop signal trials. There were 64 trials in one block and 5 blocks in total. Among the 320 trials, $50 \%$ were Go trials, $25 \%$ were No-Go trials while another $25 \%$ were Stop Signal trials. Between blocks, 20 second pauses were used. Both the colors of arrows and the stimulus orders were randomized (i .e. for one child black is Go while blue is No-Go/Stop signals, but for another child blue is Go while black is NoGo/Stop signals). Presentation of arrow lasted for $500 \mathrm{~ms}$ and each trial lasted for 3 seconds and the presentation of all stimuli lasted for 18 minutes in total. 
Appendix E-1: Publication by DMB - What do we know about transcranial direct current stimulation for Major Depression.
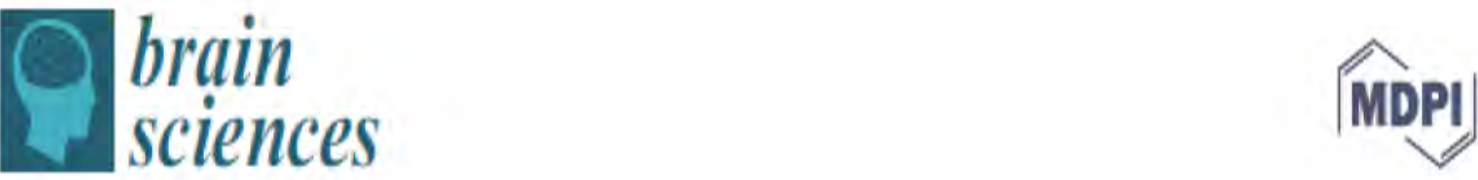

Editorial

\title{
What Do We Know about Transcranial Direct Current Stimulation for Major Depression?
}

\author{
Derrick Matthew Buchanan ${ }^{1,2,3}$, Philippe Robaey ${ }^{1,3,4}$ and Amedeo $D^{\prime}$ Angiulli ${ }^{1,2, *}$ \\ 1 Department of Neuroscience, Carleton University, Ottawa, ON K1S 5B6, Canada; \\ MatthewBuchanan@cmail.carleton.ca (D.M.B.); PRobaey@cheo.on.ca (P.R.) \\ 2 Neuroscience of Imagination Cognition Emotion Research Lab, Carleton University, \\ Ottawa, ON K1S 5B6, Canada \\ 3 Neuropsychiatry Lab, Children's Hospital of Eastern Ontario Research Institute, \\ Ottawa, ON K1H 8L1, Canada \\ 4 Department of Psychiatry, University of Ottawa, Ottawa, ON K1N 6N5, Canada \\ * Correspondence: amedeo.dangiulli@carleton.ca; Tel.: +1-613-520-2600-2954
}

Received: 21 July 2020; Accepted: 23 July 2020; Published: 25 July 2020

check for updates

\begin{abstract}
The interest in using non-invasive brain stimulation (NIBS) for the treatment of major depression (MD), including treatment resistant depression, is growing rapidly. The paper by Bennabi and Haffen (Brain Sci. 2018, 8) was an important step towards the formal acceptance of transcranial direct current stimulation (tDCS) as a possible form of therapy. Their review demonstrated favourable support for the beneficial effects of $\mathrm{tDCS}$ for MD, coupled with necessary practical considerations, such as its relatively low cost, portability/ease of use in clinical settings, non-invasiveness, and good tolerability. Here, we provide a follow-up to their review and sketch a current update. Means for optimizing tDCS efficacy and potential limitations of current studies are discussed.
\end{abstract}

Keywords: transcranial direct current stimulation; tDCS; non-invasive brain stimulation; NIBS; transcranial magnetic stimulation; transcranial magnetic stimulation (TMS); major depression 
The interest in using non-invasive brain stimulation (NIBS) for the treatment of major depression (MD), including treatment resistant depression is growing rapidly. The paper by Bennabi and Haffen [1] contributed an important assessment for the formal acceptance of transcranial direct current stimulation (tDCS) as a possible form of therapy. Their review demonstrated favorable support for the beneficial effects of tDCS for MD (effect size 0.35), but weak support for treatment resistant depression. This was coupled with a report on necessary practical considerations such as its relatively low cost, portability/ease of use in clinical settings, non-invasiveness, and its good tolerability. With the widespread uptake and now largely regulated transcranial magnetic stimulation (TMS) for MD, it seems only logical that TDCS may be the next up. At the moment, tDCS has a level B recommendation for treating MD [1,2]. Here, we provide a follow up to Bennabi and Haffen [1] and sketch a current update.

While the efficacy of tDCS is being investigated in a broad array of psychiatric and neurological conditions [3,4], its potential in major depression is among the most promising [1]. Boggio et al [5] and others [6] have presented strong evidence for tDCS efficacy in major depression; with a significant decrease in depressive symptoms ranging from $24.8-40-4 \%$ in active conditions compared to $10.4-15.9 \%$ in sham. That being said, in the decade since these early clinical trials the results have proven to be inconsistent. According to recent meta-analyses $[7,8]$ it is true that subjects generally experience a decrease on depression scales, however this reduction is rarely statistically different than sham. This is further evidenced by an international randomized controlled trial of 130 participants which found no antidepressant difference between active and sham tDCS [9]. In addition to the possibility of treatment resistant patients skewing data, there are a few leading explanations for these discrepancies in the literature.

One of the current hypotheses in the tDCS literature [10-12], which is concurrent with TMS literature [13], is that tDCS dose, such as electrode montage and other stimulation parameters need to be individualized in order to optimize efficacy. This is further based on computational MRI evidence $[14,15]$ that demonstrated how individual brain and skull morphology/density can influence current flow. Therefore, the efficacy of studies with a singular electrode location and static amperage for all subjects may be limited. To mitigate this, it is proposed that current flow should be optimized by using MRI or EEG [16]. This ensures that the optimal amount of current is reaching the region of interest for each individual subject. This is akin to how TMS clinics optimize dose response curves by locating the M1 hotspot by measuring the threshold of the motor evoked response (MEP).

Another potential explanation for the inconsistent results of tDCS for MD is the various subtypes and endophenotypes that have been identified for MD [17,18]. In 2017 Drysdale et al [17] published an article which identified four unique dysfunctional neurophysiological subtypes of MD. This was based on a multi-site sample of 1188 subjects who underwent fMRI. Connectivity analyses differentiated patients by limbic and frontostriatal networks into four biotypes with $82-93 \%$ sensitivity and specificity. The same study further demonstrated in a subsample of 154 subjects that these biotypes also predicted responsiveness to TMS. Therefore, tailoring tDCS protocols to these subtypes and other individualized biomarkers should similarly yield better outcomes.

Although current evidence suggests weak antidepressant effects of tDCS in treatment resistant groups [1] it still remains a hopeful avenue [19]. Patients with treatment resistant 
depression will try at least 2-4 medications before finding one that is right for them; or finding out that they are resistant to all of them. Given that the effects of medication can take weeks to manifest, this process is a major burden on the patient. Moreover, pharmaceuticals come with a long list of undesirable side effects. It is crucial to provide these patients with alternative treatment options that may be more suitable to their needs. In a study comparing the acceptability of tDCS to pharmaceuticals, subjects were consistently partial to tDCS [20]. Interestingly, this is also the case in children [20]. Therefore, the community is prepared to embrace the uptake of tDCS if the efficacy is established. The onus is on the researchers and clinicians to advance the optimization of tDCS in order to increase its efficacy.

In general, the non-invasive nature of tDCS, and its overall lack of side effects make it an appealing option for treating children and adolescents for the same conditions as considered in adults [21]. For parents, tDCS is a treatment option that offers hope for a short term intensive treatment option, compared to the prospect of years of being medicated [20]. That being said, tDCS studies in children are mostly limited to treating neurodevelopmental conditions like attention deficit hyperactivity disorder (ADHD), autism, or cerebral palsy (CP) [22]. tDCS studies on pediatric depression are scarce [23]. There have however been a handful of open label TMS studies that demonstrated encouraging evidence for treating pediatric depression, including treatment resistant depression, and suicidality $[24,25]$.

It is also well known that medication use can influence cortical excitability and that this may interact with the effects of tDCS [26]. Therefore, it is possible that medication may either facilitate or interfere with the antidepressant effects of tDCS. It is likely that either facilitation or interference could occur depending on the other stimulation parameters and individual differences. Studies should carefully control for this, and special consideration should be made for tDCS protocols including concomitant medication.

Finally, use of NIBS is not restricted to clinical settings. It may also be more convenient for patients to receive their treatment remotely from the comfort of their home. Indeed, several treatment studies $[28,29]$ and multiple guidelines $[30,31]$ for remotely supervised and at home tDCS have been published. In a recent pilot trial of remotely supervised tDCS for major depression patients saw a significant improvement from baseline up to one month after their treatment ended [32]. This is particularly important in the current climate of COVID-19. A recent guideline was published by Bikson et al [27] to outline the necessity and importance of continuing NIBS treatments and the steps to doing this safely during the pandemic.

In conclusion, there still appears to be a split in the literature regarding the efficacy of tDCS for treating major depression. It is possible that the heterogeneity of results is due to unspecific tDCS protocols. Such as with TMS, it is expected that the optimization of tDCS protocols by consideration of disease subtype, endophenotype, and individual neuroimaging should increase the efficacy of tDCS.

Conflicts of Interest: The authors declare no conflict of interest. 


\section{References}

1. Bennabi, D.; Haffen, E. Transcranial direct current stimulation (tDCS): A promising treatment for major depressive disorder? Brain Sci. 2018, 8, 1-10, doi:10.3390/brainsci8050081.

2. Lefaucheur, J.P.; Antal, A.; Ayache, S.S.; Benninger, D.H.; Brunelin, J.; Cogiamanian, F.; Cotelli, M.; De Ridder, D.; Ferrucci, R.; Langguth, B.; et al. Evidence-based guidelines on the therapeutic use of transcranial direct current stimulation (tDCS). Clin. Neurophysiol. 2017.

3. Tortella, G.; Casati, R.; Aparicio, L.V.M.; Mantovani, A.; Senço, N.; D’Urso, G.; Brunelin, J.; Guarienti, F.; Selingardi, P.M.L.; Muszkat, D.; et al. Transcranial direct current stimulation in psychiatric disorders. World J. psychiatry 2015, 5, 88-102, doi:10.5498/wjp.v5.i1.88.

4. Rivera-Urbina, G.N.; Nitsche, M.A.; Vicario, C.M.; Molero-Chamizo, A. Applications of transcranial direct current stimulation in children and pediatrics. Rev. Neurosci 2017, 28 , 173-184, doi:10.1515/revneuro-2016-0045.

5. $\quad$ Fregni, F.; Boggio, P.S.; Nitsche, M. a; Marcolin, M. a; Rigonatti, S.P.; Pascual-Leone, A. Treatment of major depression with transcranial direct current stimulation. Bipolar Disord. 2006, 8, 203-204, doi:10.1111/j.1399-5618.2006.00291.x.

6. Loo, C.K.; Sachdev, P.; Martin, D.; Pigot, M.; Alonzo, A.; Malhi, G.S.; Lagopoulos, J.; Mitchell, P. A double-blind, sham-controlled trial of transcranial direct current stimulation for the treatment of depression. Int. J. Neuropsychopharmacol. 2010, doi: $10.1017 /$ S1461145709990411.

7. Berlim, M.T.; Van den Eynde, F.; Daskalakis, Z.J. Clinical utility of transcranial direct current stimulation (tDCS) for treating major depression: A systematic review and metaanalysis of randomized, double-blind and sham-controlled trials. J. Psychiatr. Res. 2013, 47, 1-7, doi:10.1016/j.jpsychires.2012.09.025.

8. Razza, L.B.; Palumbo, P.; Moffa, A.H.; Carvalho, A.F.; Solmi, M.; Loo, C.K.; Brunoni, A.R. A systematic review and meta-analysis on the effects of transcranial direct current stimulation in depressive episodes. Depress. Anxiety 2020, da.23004, doi:10.1002/da.23004.

9. Loo, C.K.; Husain, M.M.; McDonald, W.M.; Aaronson, S.; O’Reardon, J.P.; Alonzo, A.; Weickert, C.S.; Martin, D.M.; McClintock, S.M.; Mohan, A.; et al. International randomized-controlled trial of transcranial Direct Current Stimulation in depression. Brain Stimul. 2018, 11, 125-133, doi:10.1016/j.brs.2017.10.011.

10. Esmaeilpour, Z.; Marangolo, P.; Hampstead, B.M.; Bestmann, S.; Galletta, E.; Knotkova, H.; Bikson, M. Incomplete evidence that increasing current intensity of tDCS boosts outcomes. Brain Stimul. 2018, 11, doi:10.1016/j.brs.2017.12.002.

11. Kessler, S.K.; Minhas, P.; Woods, A.J.; Rosen, A.; Gorman, C.; Bikson, M. Dosage Considerations for Transcranial Direct Current Stimulation in Children: A Computational Modeling Study. PLoS One 2013, 8, 1-15, doi:10.1371/journal.pone.0076112.

12. Jamil, A.; Batsikadze, G.; Kuo, H.I.; Labruna, L.; Hasan, A.; Paulus, W.; Nitsche, M.A. Systematic evaluation of the impact of stimulation intensity on neuroplastic after-effects induced by transcranial direct current stimulation. J. Physiol. 2017, 595, doi:10.1113/JP272738.

13. Dunlop, K.; Gaprielian, P.; Blumberger, D.; Daskalakis, Z.J.; Kennedy, S.H.; Giacobbe, P.; Downar, J. MRI-guided dmPFC-rTMS as a Treatment for Treatment-resistant Major 
Depressive Disorder. J. Vis. Exp. 2015, e53129, doi:10.3791/53129.

14. Truong, D.Q.; Magerowski, G.; Blackburn, G.L.; Bikson, M.; Alonso-Alonso, M.

Computational modeling of transcranial direct current stimulation (tDCS) in obesity: Impact of head fat and dose guidelines. NeuroImage Clin. 2013, 2, 759-766, doi:10.1016/j.nicl.2013.05.011.

15. Edwards, D.; Cortes, M.; Datta, A.; Minhas, P.; Wassermann, E.M.; Bikson, M. Physiological and modeling evidence for focal transcranial electrical brain stimulation in humans: A basis for high-definition tDCS. Neuroimage 2013, 74, 266-275, doi:10.1016/j.neuroimage.2013.01.042.

16. Ruffini, G.; Fox, M.D.; Ripolles, O.; Miranda, P.C.; Pascual-Leone, A. Optimization of multifocal transcranial current stimulation for weighted cortical pattern targeting from realistic modeling of electric fields. Neuroimage 2014, doi:10.1016/j.neuroimage.2013.12.002.

17. Drysdale, A.T.; Grosenick, L.; Downar, J.; Dunlop, K.; Mansouri, F.; Meng, Y.; Fetcho, R.N.; Zebley, B.; Oathes, D.J.; Etkin, A.; et al. Resting-state connectivity biomarkers define neurophysiological subtypes of depression. Nat. Med. 2017, 23, 28-38, doi: $10.1038 / \mathrm{nm} .4246$.

18. Webb, C.A.; Dillon, D.G.; Pechtel, P.; Goer, F.K.; Murray, L.; Huys, Q.J.M.; Fava, M.; McGrath, P.J.; Weissman, M.; Parsey, R.; et al. Neural Correlates of Three Promising Endophenotypes of Depression: Evidence from the EMBARC Study.

Neuropsychopharmacology 2016, doi:10.1038/npp.2015.165.

19. Al-Harbi, K.S. Treatment-resistant depression: Therapeutic trends, challenges, and future directions. Patient Prefer. Adherence. 2012.

20. Buchanan, D.M.; Samson, A.; Maisonneuve R, A.; D’Angiulli, A.; Robaey, P. Acceptability of transcranial direct current stimulation in children and adolescents with ADHD: The point of view of parents. J. Health Psychol. 2020, doi.org/10.1177/1359105320937059.

21. Buchanan, D.; D’Angiulli, A.; Samson, A.; Amare, S.; Gaumond, G.; Robaey, P. Making transcranial direct current stimulation treatment in atypical child and adolescent neurodevelopment a reality: Translating safety tolerability and acceptability evidence from the laboratory into the doctors office, the classroom, and home. Brain Stimul. 2019, 12, 474, doi:10.1016/j.brs.2018.12.545.

22. Rivera-Urbina, G.N.; Nitsche, M.A.; Vicario, C.M.; Molero-Chamizo, A. Applications of transcranial direct current stimulation in children and pediatrics. Rev. Neurosci. 2017, 28 , 173-184, doi:10.1515/revneuro-2016-0045.

23. Lee, J.C.; Lewis, C.P.; Daskalakis, Z.J.; Croarkin, P.E. Transcranial direct current stimulation: Considerations for research in adolescent depression. Front. Psychiatry 2017, 8 .

24. Lewis, C.P.; Farzan, F.; Croarkin, P.E. TMS in child and adolescent major depression. In Neurotechnology and Brain Stimulation in Pediatric Psychiatric and Neurodevelopmental Disorders; 2018 ISBN 9780128127773.

25. Croarkin, P.E.; MacMaster, F.P. Transcranial Magnetic Stimulation for Adolescent Depression. Child Adolesc. Psychiatr. Clin. N. Am. 2019.

26. McLaren, M.E.; Nissim, N.R.; Woods, A.J. The effects of medication use in transcranial direct current stimulation: A brief review. Brain Stimul. 2018, 11.

27. Bikson, M.; Hanlon, C.A.; Woods, A.J.; Gillick, B.T.; Charvet, L.; Lamm, C.; Madeo, G.; 
Holczer, A.; Almeida, J.; Antal, A.; et al. Guidelines for TMS/tES clinical services and research through the COVID-19 pandemic. Brain Stimul. 2020, doi:10.1016/j.brs.2020.05.010.

28. Charvet, L.; Shaw, M.; Dobbs, B.; Frontario, A.; Sherman, K.; Bikson, M.; Datta, A.; Krupp, L.; Zeinapour, E.; Kasschau, M. Remotely Supervised Transcranial Direct Current Stimulation Increases the Benefit of At-Home Cognitive Training in Multiple Sclerosis. Neuromodulation 2018, doi:10.1111/ner.12583.

29. André, S.; Heinrich, S.; Kayser, F.; Menzler, K.; Kesselring, J.; Khader, P.H.; Lefaucheur, J.P.; Mylius, V. At-home tDCS of the left dorsolateral prefrontal cortex improves visual short-term memory in mild vascular dementia. J. Neurol. Sci. 2016, doi:10.1016/j.jns.2016.07.065.

30. Charvet, L.E.; Kasschau, M.; Datta, A.; Knotkova, H.; Stevens, M.C.; Alonzo, A.; Loo, C.; Krull, K.R.; Bikson, M. Remotely-supervised transcranial direct current stimulation (tDCS) for clinical trials: guidelines for technology and protocols. Front. Syst. Neurosci. 2015, 9, 26, doi:10.3389/fnsys.2015.00026.

31. Charvet, L.E.; Shaw, M.T.; Bikson, M.; Woods, A.J.; Knotkova, H. Supervised transcranial direct current stimulation (tDCS) at home: A guide for clinical research and practice. Brain Stimul. 2020, doi:10.1016/j.brs.2020.02.011.

32. Alonzo, A.; Fong, J.; Ball, N.; Martin, D.; Chand, N.; Loo, C. Pilot trial of homeadministered transcranial direct current stimulation for the treatment of depression. $J$. Affect. Disord. 2019, doi:10.1016/j.jad.2019.04.041. 
Appendix F - 1: Publication by DMB - Commercial wireless versus standard stationary EEG systems for personalized emotional brain-computer interfaces: a preliminary reliability check.

NEUROSCIENCE RESEARCH NOTES

\title{
Commercial wireless versus standard stationary EEG systems for personalized emotional brain-computer interfaces: a preliminary reliability check
}

Derrick Matthew Buchanan ${ }^{1,2}$, Jeremy Grant ${ }^{1,2}$ and Amedeo D'Angiulli 1,2,*

${ }^{1}$ Neuroscience of Imagination Cognition Emotion Research Laboratory, Ontario, Canada.

${ }^{2}$ Department of Neuroscience, Carleton University, Ontario, Canada.

* Correspondence: amedee daneiulli@carleton.ca; Tel.: +1-613-520-2600 × 2954

Received: 15 November 2018; Accepted: 5 March 2019; Published: 11 March 2019

Edited by: King-Hwa Ling (Universiti Putra Malaysia, Malaysia)

Reviewed by: Bingzhe Xu (Harvard Medical School, Boston, USA); Norshariza Nordin (Universiti Putra Malaysia); Dania Cheaha (Prince of Songkla University, Thailand)

https://doi.org/10.31117/neuroscirn.v/2n1.21

\begin{abstract}
We present a preliminary data-based assessment of measurement reliability of the commercial 14-electrode Emotiv EPOCTM EEG wireless system in distinguishing between electrophysiological states of emotional function, as compared to a standard research-lab stationary 32-electrode EEG system for personalized single-individual use. Individual observers completed two tasks designed to elicit neural changes in emotional arousal and valence while simultaneously recording their EEGs with both systems in separate sessions. Participants observed emotion-laden words from the ANEW database and images from the IAPS database, both widely used and validated databases for emotional processes in multidisciplinary research. The pattern of results distinguished between high and low arousal and valence states using the stationary traditional system, but not the commercial device. Also, the latter device recorded EEG band frequencies at a much lower resolution and frequency range than the standard system. These findings suggest poor validity when using the commercial device and therefore should be cautioned against in a research setting.
\end{abstract}

Keywords: arousal; valence; brain-computer Interfaces; EEG; wireless;

(92019 by Buchanan et al. for use and distribution in accord with the Creative Commons Attribution (CC BY-NC 4.0) license (https://creativecommons.ore/licenses/by-nc/4.0/), which permits unrestricted non-commercial use, distribution, and reproduction in any medium, provided the original author and source are credited. 


\section{Introduction}

The identification and classification of human emotions using electroencephalography (EEG) is major research focus for developing Brain-Computer Interface (BCI) devices. Translating electrophysiological signals from a person's scalp into quantifiable data (event-related potentials, or ERPs) is useful for programming computer systems to recognize emotional content in facial expressions and vocal inflexions [1] and allows software-based intelligence systems to adapt their function to a user's overall emotional state in real-time [2]. The need for cost-effective EEG systems that can reliably distinguish between emotional states is growing in importance; EEG systems with a greater number of electrodes enable researchers to provide higher resolution recordings of electrophysiological activity, but also require a lengthy preparation time to arrange the electrodes and test the signal amplification. Public and private businesses seeking to employ BCI devices to enhance their operatives' functionality are often interested in EEG systems with fewer electrodes-minimizing preparation time while potentially sacrificing the quality and resolution of the electrophysiological signal recordings. Recent reports have demonstrated that there is indeed some research utility for consumer grade devices such as the Epoc as compared to other similar devices such as the Neurosky MindWave [3], however both of these devices' performance are questionable compared to proper research grade EEG [4]. The push to incorporate classification algorithms within these devices is prominently associated with the alluring promise of personalization, single-individualized use according to which the $\mathrm{BCI}$ system might be calibrated and fine-tuned to the individual characteristics of the persons brain/mind (which assumes an " $\mathrm{N}=1$ " approach). Our current investigation provides novel evidence against relying on consumer devices for research grade tasks, specifically in this case for measuring neuropsychological aspects of emotional states (stimulus valence and arousal). Most research using consumer grade devices are limited using only the consumer device itself; failing to demonstrate validity and reproducibility of results using a juxtaposed research grade device. We overcome this limitation by directly comparing the data acquired from a consumer grade EEG to that of a research grade EEG under the same conditions using a within subject design.

We acquired two adjacent EEG systems for their potential application as a part of a brain-computer interface: the Brain Vision Easy Cap EC40 headset and the actiCHamp active channel amplifier system (henceforth Brain Vision) as well as the Emotiv EPOCTM EEG wireless system (henceforth abbreviated simply as Epoc), The Brain Vision Easy Cap is a standard research grade 32-electrode headset, whereas the Epoc headset is a consumer grade device with only 14 electrodes, but boasts a much more elegant user-friendly design. In addition, it transmits electrophysiological data wirelessly via a BluetoothTM transmitter, bypassing the signal processing traditionally performed by an external amplifier. While preparation time is minimal (10-15 min on average, as compared to 45-60 minutes for BV), the EPOC headset does not have any electrodes placed along the midline of the head, and it uses two reference electrodes on both sides of the head corresponding with the parietal lobe (P3 and P4), as opposed to using a single ground electrode along the midline as reference in traditional EEG systems. Furthermore, the electrodes are placed on the scalp without any gel substance serving as an intermediate for electrical conduction, thereby further limiting the ability to perform EEG source 
analysis (approximating the anatomical source of temporal electrical activity). For this reason, a viable test of comparative measurement reliability with other systems can be done through measurement of global or whole electrical brain activity. This is the approach we took in this preliminary study.

In this study, we evaluated the Brain Vision Easy Cap EC40 and the Epoc EEG systems on their efficacy and reliability in differentiating between varying levels of two distinct dimensions of emotion, emotional arousal and valence [4]. We presented human subjects with two experimental tasks designed to examine if the Brain Vision system could be used to distinguish between states of high and low arousal and high and low valence, based on patterns of event-related frequency band potentials (ERBPs) in response to the presentation of emotionally-salient words or visual stimuli. Importantly, we report novel findings regarding the comparable signal quality of each device and the inability of Epoc to reproduce the results seen using the BV device.

\section{Materials and Methods}

\subsection{Participants}

Participants were three healthy graduate and undergraduate students (mean age 24.66 years old) serving as trained research assistants. This study followed all ethical guidelines on human participant set out by the host institution. All participants were righthanded males with corrected-to-normal $(\mathrm{N}=2)$ or normal $(\mathrm{N}=1)$ vision, and none reported any history of neurological impairment or were currently using psychoactive medications. The average number of hours of sleep on the previous night was 7.5 hours. Testing was conducted from 10 a.m. to 4 p.m. with a one-hour break in between on two separate days. Pre-experimental short adult version of the mood and feelings questionnaire (Messer, Angold, Costello, Loeber,Van Kammen, \& Stouthamer-Loeber, 1995) were administered by an independent research assistant unaware of the hypotheses and goals of the study to screen mood differences or emotional changes before the experimental sessions between days. No remarkable differences were reported as all participants scored similarly (overall score range: $3-5$ ) in the two days and consistently well below the recommended clinical cut-off (possible maximum $=26$; clinical cut-off $=11$ ).

\subsection{Experimental Design}

The participants completed two tasks sitting in front of a computer monitor while undergoing ERP analysis using the 32-electrode Brain Vision EEG system, and repeating both tasks two days later while using the 14-electrode Epoc EEG system.

In the first task, subjects observed a series of selected images from the International Affective Picture System (IAPS). IAPS is a large standardized database of images, where each image carries a rating based on its three dimensions of emotion: valence, arousal, and control/dominance [6]. Emotion-laden pictures that had previously been categorized by the target parameters of valence and arousal were used to designate the image as high or low arousal/valence, previously shown to correlate with known EEG signatures [7]. Similar to this research, a recent fMRI study has shown that the emotions evoked by these images effectively separate the emotions into distinct arousal/valence quadrants [8]. The calibration for these emotion-laden localizations occurred across participants. We pre-selected 100 high arousal and 100 low arousal images (high and low arousal determined by their rating in the database) as well as 100 high valence and 100 
low valence images for use in our study. We presented each participant with a randomized selection of 100 images ( 25 high arousal, 25 low arousal, 25 high valence, 25 low valence) on a computer monitor while recording their electrophysiological activity via the Brain Vision headset. The stimulus presentation program began with a central cross fixation to help participants focus their gaze and reduce eye movements. After $200 \mathrm{~ms}$, the first image was presented for $4500 \mathrm{~ms}$, followed by a $1300 \mathrm{~ms}$ presentation of the cross fix preceding the second image. Participants were specifically instructed to try to form emotional connection with each image/word presented, and to remain as still as possible while viewing images/words. Images filled a $48.3 \mathrm{~cm}$ (19") monitor with a 1024 x 768 pixel resolution. Letters were presented at a font size of 48 points and presented in the center of the monitor.

In the second task, subjects observed a series of selected words from the Affective Norms for English Words (ANEW) database. ANEW is another standardized inventory of normative emotional ratings for a large number of words in the English language; it was developed to complement IAPS [9]. Previous research using the ANEW has plotted the normative ratings in 2-dimensional space and found the distribution effectively complements the IAPS using EEG [8]. We presented each participant with a selection of words from the ANEW database on a computer monitor while recording their electrophysiological activity (the same procedure as with the IAPS images, this time with words); one series of words with the Brain Vision headset, and another series with the Epoc headset.

\subsection{Event-Related Band Potentials \& Signal Amplification}

EEGs were recorded for each participant during the tasks. The Brain Vision EEG recordings were obtained from electrodes held in place by means of an elastic cap (Easy Cap, Brain Vision, Morrisville, NC, USA) adhering to the ten-twenty system of measurements [10] with 32 active low-noise pin-type $\mathrm{Ag}-\mathrm{AgCl}$ electrodes ( $1 \mathrm{~mm}$ each) amplified (sampled up to $1000 \mathrm{~Hz}$ ) and recorded reference-free. Standard external landmarks (i.e. the inion, the nasion, and left and right pre-auricular points) were the basis for the montage distribution with electrodes positioned as percentage distances from these landmark points. All impedances were kept under $25 \mathrm{kOhms}$ so that they provided effective electrical signal with minimal noise. The Epoc recordings were collected as is with no additional materials or measurements other than the device itself.

Electrophysiological signals were amplified and low pass filtered at $100 \mathrm{kHz}$ via the actiCHamp active channel amplifier with a sampling rate of $1000 \mathrm{~Hz}$. All electrodes were offline re-referenced to a common average reference (i.e. the average was subtracted from each electrode for each time point). Offline averaging was performed such that event-related frequency band potentials (ERBP) were averaged separately for each stimulus type and condition for each electrode with an Epoch of $-200 \mathrm{~ms}$ pre-stimulus to $1000 \mathrm{~ms}$ post-stimulus. ERBPs included the individual trials to code high and low arousal, high and low valence.

\subsection{Statistical Analysis}

For our statistical analysis it was necessary to evaluate whole brain ERBP because the precision of the electrode placement in the Epoc could not be confirmed. Hence, there is no validity without this and comparing electrodes of one system with the other is 
fruitless. Additionally, we used a Vincentization technique for the EEG data which involves the averaging of three or more subjects' quantile functions in order to obtain group quantiles [13-15]. This statistical technique allowed us to construct $F$ values to evaluate differences between the five frequency bands.

\section{Ethics Statement}

Upon review of the submission materials, following Article 2.1 the Tri-Council Policy Statement Carleton University Research Ethics Board determined that this study did not require prior review or approval by the Board documentation and signed consent was waived. Document of consent was obtained by recording computerized log-ins during voluntary participation.

\section{Results}

\subsection{Distinguishing Electrophysiological States of Arousal and Valence with the} ANEW Task

The two panels below (Figure 1) summarize the frequency band analysis for examining arousal and valence using the ANEW task. We plotted the means of the frequency band amplitude, measured in microvolts $(\mu \mathrm{V})$ averaged across participants and electrodes. Figure 1A displays the comparison between high and low arousal states using the Brain Vision system and the Epoc system. A two-way analysis of variance revealed that the observed differences in the arousal condition (high arousal vs. low arousal) was not statistically significant for the Brain Vision system $(F(1,31)=0.085 ; p=0.772)$ nor the Epoc system $(\mathrm{F}(1,13)=7.185, \mathrm{p}=0.019)$; differences in frequency band (Delta, Theta, Alpha, Beta, Gamma) were significant for the Brain Vision system only $(\mathrm{F}(1,31)=$ $34.156, \mathrm{p}<0.01)$; and the interaction between arousal condition and frequency was significant for the Brain Vision system $(\mathrm{F}(1,31)=53.339, \mathrm{p}<0.01)$ but not the Epoc system $(F(1,13)=2.191, p=1.54)$. Figure $1 \mathrm{~B}$ displays the high and low valence states using the Brain Vision and Emotiv system. A two-way analysis of variance revealed that the observed differences in condition (high valence vs. low valence) was not statistically significant for either system; differences in frequency band (Delta, Theta, Alpha, Beta, Gamma) were significant for the Brain Vision system only $(F(1,31)=26.690, p<0.01)$; and the interaction between valence condition and frequency was significant for the Brain Vision system only $(\mathrm{F}(1,31)=40.048, \mathrm{p}<0.01)$.

\subsection{Distinguishing Electrophysiological States of Arousal and Valence with the IAPS Task}

The two panels below (Figure 2) summarize the frequency band analysis for examining arousal and valence using the IAPS task. Figure 2A displays the distinguishing of high and low arousal states using the Brain Vision system and Epoc system. A twoway analysis of variance revealed that the observed differences in the arousal condition (high arousal vs. low arousal) was not statistically significant for either system; differences in frequency band (Delta, Theta, Alpha, Beta, Gamma) were significant for the Brain Vision system only $(\mathrm{F}(1,31)=63.224, \mathrm{p}<0.01)$; and the interaction between arousal condition and frequency was also significant for Brain Vision only $(\mathrm{F}(1,31)=$ $12.303, \mathrm{p}<0.01)$. Figure $2 \mathrm{~B}$ displays the comparison between high and low valence states using the Brain Vision system and the distinguishing of high and low valence states using 
the Epoc system. A two-way analysis of variance revealed that the observed differences in the valence condition (high valence vs. low valence) was statistically significant for the Brain Vision system only $(\mathrm{F}(1,31)=19.157, \mathrm{p}<0.01$,); differences in frequency band (Delta, Theta, Alpha, Beta, Gamma) were significant for Brain Vision only $(\mathrm{F}(1,31)=$ $72.550, \mathrm{p}<0.01)$; and the interaction between valence condition and frequency was also significant for Brain Vision $(\mathrm{F}(1,31)=51.138, \mathrm{p}<0.01)$.

\subsection{Alerting Correlations of Epoc and BV}

Correlational data (Table $1 \& 2$ ) below provided some alerting trends among the different systems and tasks. Here we used the approach outlined by Rosnow, Rosenthal, and Rubin (2000) which describes the usefulness of leveraging aggregate correlations of group means rather than individual scores for identifying overall trends and assessing validity [16]. Using this alerting correlation method we identified trends among ERBP and the different task conditions. In both Table 1 and Table 2 it can be noted that the Epoc ERBP has a very poor and often inverse relationship to that of the BV which questions the validity of the Epoc.

\section{Discussion}

The results of this perspective study suggest that the wireless 14-electrode Epoc system can be used to show small differences in general electrical activity between psychophysiological states of high and low arousal, and high and low valence (particularly in the delta, theta, and alpha frequency ranges); but the observed differences are not statistically significant such as with the stationary Brain Vision device. Therefore, the use of the Epoc device definitely lacks the degree of fidelity that is required to properly distinguish these states within a personalized design which would be very undesirable in a research setting. The findings offer evidence against the validity of consumer grade systems for research grade tasks, although their low fidelity ability to distinguish states may still be useful for some EEG-based BCI applications. The Epoc, and similar consumer grade devices with a limited number of electrodes, could be strongly improved simply by designing a product which adheres to conventional EEG electrode placement. Using the common electrode placements would lead to improved comparative capabilities with traditional EEG devices/existing EEG studies (eg. Meta-analysis), better referencing montages, and increased translatability of anatomical related conclusions.

During the ANEW and IAPS tasks where the Brain Vision system detected high levels of gamma frequency band activity, the Epoc system consistently failed to replicate this finding. We hypothesize that this difference can be attributed to technical aspects of the 14-electrode setup such as the lack of a signal amplifier. On the other hand, the Brain Vision system uses an ActiChampTM signal amplifier which enables it to detect higher frequencies of electrophysiological activity - higher beta oscillations and gamma oscillations. In contrast, the Epoc wireless headset transmits electrophysiological recordings directly from the scalp to a USB-A dongle in the signal processing computer. This interface for amplifying signals may be in part responsible for making higher beta and gamma oscillations more difficult to detect. It goes without saying that more sophisticated amplification would drastically improve the signal quality of consumer devices, but the cost required to do so would not be consumer friendly.

For the ANEW task, differences in frequency band amplitude between high and 
low valence states were not significant, whereas the interaction between valence condition and frequency was significant. This discrepancy is likely due to the close proximity of the emotion-evoking ratings in the high valence and low valence conditions. Future studies should examine this result using a greater difference between the mean rating of the words used in the two conditions of the ANEW task.

In conclusion, we demonstrate further evidence for a consumer EEGs' inability to reproduce differences that are distinctly obtainable using a research EEG device [17]. We are able to demonstrate this with a small number of subjects by using the statistical technique of Vincentization. Given that the goal of most emerging approaches using EEGbased machine learning and categorization algorithms is ultimately intended for a personalized individual consumer use, the fact that the present perspective focused on a demonstration based on a very small sample cannot be considered as a major limitation. The experiment was done at the same grade (scale) as that of the intended consumer usage: in other words, it focusses on usability in one person, not groups of people.

Overall, we urge caution to researchers planning to use a consumer device for important research experiments, but do acknowledge that devices like the Emotiv have some benefits such as being wireless, quick to set up, are extremely portable, and could still be useful in some BCI applications where signal quality and validity are not paramount. For instance, exerting BCI control over an external device (eg. Wheelchair, prosthetic arm) can be done by calibrating any signal to a function of the device (eg. Move forward, backward, side to side, extend/retract reach). Notice, in this example the ability for the BCI to differentiate valence/arousal emotional states was not necessary. In this case the goal is to make the system successfully work for the user, not to produce valid signatures of emotion. On the other hand, if the goal of a study is anything to do with emotional states, like using an Epoc to investigate clinical neuropsychiatric populations for emotional dysregulation in mood disorders, or any cognitive neuroscience experiment of emotion in which you need to be confident in the validity of your signal, the consumer grade devices must be cautioned against. Therefore, we argue that the issue is not so much that consumer devices have no place in the research laboratory, but rather that their use and data interpretation need to be done appropriately in contexts in which they are valid. Upon this investigation, using an Epoc EEG device to determine emotional states in individuals would have questionable validity as it is not reproducible using research grade EEG. The validity should not be assumed as simply coming in a box, together with the device!

Supplementary Materials: Additional information regarding the EEG signal processing are available online at www.neuroscirn.org/link.

Acknowledgements: This work was funded by a NSERC/DND partnership grant (Natural Sciences and Engineering Research Council and Department of National Defense of Canada) to $\mathrm{AD}$. No funds were received for covering the costs to publish in open access.

Author Contributions: DMB and JG contributed equally to this paper and were responsible for performing analysis of data and the writing of the paper. $\mathrm{AD}$ conceived, designed, and oversaw the experiments, and revised and edited paper. JG and other assistants performed the experiment. 
Conflicts of Interest: "The authors declare no conflict of interest"

\section{References}

1. Lin, Y. P., Wang, C. H., Jung, T. P., Wu, T. L., Jeng, S. K., Duann, J. R., \& Chen, J. H. (2010). EEG-based Emotion Recognition. IEEE Transactions on Biomedical Engineering. https://doi.org/10.1109/TBME.2010.2048568

2. Ross, W., Morris, A., Ulieru, M., \& Guyard, A. B. RECON: An adaptive human-machine system for supporting intelligence analysis. IEEE International Conference on Systems, Man, and Cybernetics, 782-787 (2013)

3. Maskeliunas, R., Damasevicius, R., Martisius, I., \& Vasiljevas, M. (2016). Consumergrade EEG devices: are they usable for control tasks? PeerJ, 4, e1746. https://doi.org/10.7717/peerj.1746

4. Duvinage, M., Castermans, T., Petieau, M., Hoellinger, T., Cheron, G., \& Dutoit, T. (2013). Performance of the Emotiv Epoc headset for P300-based applications. BioMedical Engineering OnLine, 12(1), 56. https://doi.org/10.1186/1475-925X-12-56

5. Lang, P. J., Greenwald, M. K., Bradley, M. M., \& Hamm, a O. Looking at pictures: affective, facial, visceral, and behavioral reactions. Psychophysiology, 30, 261-273. (1993)

6. Paas, F., Tuovinen, J. E., Tabbers, H., \& Van Gerven, P. W. M. Cognitive Load Measurement as a Means to Advance Cognitive Load Theory. Educational Psychologist, 38(1), 63-71. (2003)

7. Lang, P. J., Bradley, M. M., \& Cuthbert, B. N. International Affective Picture System (IAPS): Technical Manual and Affective Ratings. Psychology, Vol. 77 (1997)

8. Scott, G. G., O’Donnell, P. J., Leuthold, H., \& Sereno, S. C. Early emotion word processing: Evidence from event-related potentials. Biological Psychology, 80, 95-104 (2009)

9. Kassam, K. S., Markey, A. R., Cherkassky, V. L., Loewenstein, G., \& Just, M. A. Identifying Emotions on the Basis of Neural Activation. PLoS ONE, 8(6) (2013)

10. Jurcak, V., Tsuzuki, D., \& Dan, I. (2007). 10/20, 10/10, and 10/5 systems revisited: Their validity as relative head-surface-based positioning systems. NeuroImage. https://doi.org/10.1016/j.neuroimage.2006.09.024

11. Bradley, M.M., \& Lang, P.J. Affective norms for English words (ANEW): Instruction manual and affective ratings. (1999)

12. Messer, S. C., Angold, A., Costello, E.J., Loeber, R., Van Kammen, W., \& StouthamerLoeber, M. (1995). Development of a short questionnaire for use in epidemiological studies of depression in children and adolescents: Factor composition and structure across development. International Journal of Methods in Psychiatric Research, 5, 251-262.

13. Vincent, S. B. (1912). The functions of the vibrissae in the behavior of the white rat. Behavior Monographs. (Vol. 1, No. 5). University of Chicago.

14. Hilgard, ER (1951). Methods and procedures in the study of learning. In Stevens, SS (Ed.) Handbook of experimental psychology. - New York, Wiley, p.517-567.

15. Genest, C. (1992). Vincentization Revisited. The Annals of Statistics. Institute of Mathematical Statistics. (Vol. 20), 1137-1142. https://doi.org/10.2307/2242003

16. Rosnow, C., Rosenthal, R., \& Rubin, D. B. (2000). Contrasts and correlations in effect- 
size estimation. Psychological Science, 11(6), 446-453. https://doi.org/10.1111/14679280.00287

17. Nijboer, F., Van De Laar, B., Gerritsen, S., Nijholt, A., \& Poel, M. (2015). Usability of three electroencephalogram headsets for brain-computer interfaces: A within subject comparison. Interacting with Computers, 27(5), 500-511.

https://doi.org/10.1093/iwc/iwv023 
Table 1. Alerting Pearson Correlations of ERBP for each condition and EEG system (Brain Vision, BV, vs. Epoc Emotiv) using the ANEW task.

\begin{tabular}{|c|c|c|c|c|c|c|c|c|c|}
\hline & $\begin{array}{c}\text { EEG } \\
\text { Frequency }\end{array}$ & $\begin{array}{c}\text { Epoc } \\
\text { Low } \\
\text { Arousal }\end{array}$ & $\begin{array}{l}\text { Epoc High } \\
\text { Arousal }\end{array}$ & $\begin{array}{l}\text { BV Low } \\
\text { Arousal }\end{array}$ & $\begin{array}{l}\text { BV High } \\
\text { Arousal }\end{array}$ & $\begin{array}{l}\text { Epoc Low } \\
\text { Valence }\end{array}$ & $\begin{array}{l}\text { Epoc High } \\
\text { Valence }\end{array}$ & $\begin{array}{l}\text { BV Low } \\
\text { Valence }\end{array}$ & $\begin{array}{l}\text { BV High } \\
\text { Valence }\end{array}$ \\
\hline $\begin{array}{c}\text { EEG } \\
\text { Frequency }\end{array}$ & 1 & & & & & & & & \\
\hline $\begin{array}{c}\text { Epoc Low } \\
\text { Arousal }\end{array}$ & $\begin{array}{l}-0.799 \\
(0.105)\end{array}$ & 1 & & & & & & & \\
\hline $\begin{array}{l}\text { Epoc High } \\
\text { Arousal }\end{array}$ & $\begin{array}{l}-0.809 \\
(0.098)\end{array}$ & $\begin{array}{c}.992^{* *} \\
(0.001)\end{array}$ & 1 & & & & & & \\
\hline $\begin{array}{l}\text { BV Low } \\
\text { Arousal }\end{array}$ & $\begin{array}{r}0.444 \\
(0.454)\end{array}$ & $\begin{array}{r}0.099 \\
(0.874)\end{array}$ & $\begin{array}{r}0.134 \\
(0.830)\end{array}$ & 1 & & & & & \\
\hline $\begin{array}{l}\text { BV High } \\
\text { Arousal }\end{array}$ & $\begin{array}{r}0.682 \\
(0.205)\end{array}$ & $\begin{array}{l}-0.241 \\
(0.696)\end{array}$ & $\begin{array}{l}-0.202 \\
(0.745)\end{array}$ & $\begin{array}{c}.932^{*} \\
(0.021)\end{array}$ & 1 & & & & \\
\hline $\begin{array}{l}\text { Epoc Low } \\
\text { Valence }\end{array}$ & $\begin{array}{l}-0.811 \\
(0.096)\end{array}$ & $\begin{array}{r}0.790 \\
(0.112)\end{array}$ & $\begin{array}{r}0.856 \\
(0.064)\end{array}$ & $\begin{array}{r}0.040 \\
(0.949)\end{array}$ & $\begin{array}{l}-0.234 \\
(0.704)\end{array}$ & 1 & & & \\
\hline $\begin{array}{l}\text { Epoc High } \\
\text { Valence }\end{array}$ & $\begin{array}{c}-.912^{*} \\
(0.031)\end{array}$ & $\begin{array}{c}.928^{*} \\
(0.023)\end{array}$ & $\begin{array}{c}.958^{*} \\
(0.010)\end{array}$ & $\begin{array}{l}-0.037 \\
(0.953)\end{array}$ & $\begin{array}{l}-0.329 \\
(0.589)\end{array}$ & $\begin{array}{c}.926^{*} \\
(0.024)\end{array}$ & 1 & & \\
\hline $\begin{array}{l}\text { BV Low } \\
\text { Valence }\end{array}$ & $\begin{array}{r}0.424 \\
(0.477)\end{array}$ & $\begin{array}{r}0.124 \\
(0.842)\end{array}$ & $\begin{array}{r}0.159 \\
(0.798)\end{array}$ & $\begin{array}{c}.992^{* *} \\
(0.001)\end{array}$ & $\begin{array}{c}.901^{*} \\
(0.037)\end{array}$ & $\begin{array}{r}0.083 \\
(0.894)\end{array}$ & $\begin{array}{l}-0.020 \\
(0.975)\end{array}$ & 1 & \\
\hline $\begin{array}{l}\text { BV High } \\
\text { Valence }\end{array}$ & $\begin{array}{r}0.431 \\
(0.469)\end{array}$ & $\begin{array}{r}0.124 \\
(0.843)\end{array}$ & $\begin{array}{r}0.152 \\
(0.807)\end{array}$ & $\begin{array}{l}.997^{* *} \\
(0.000)\end{array}$ & $\begin{array}{c}.928^{*} \\
(0.023)\end{array}$ & $\begin{array}{r}0.021 \\
(0.973)\end{array}$ & $\begin{array}{l}-0.025 \\
(0.968)\end{array}$ & $\begin{array}{c}.982^{* *} \\
(0.003)\end{array}$ & 1 \\
\hline
\end{tabular}

*. Correlation is significant at the 0.05 level (2-tailed).

**. Correlation is significant at the 0.01 level (2-tailed).

${ }^{1}$ Exact $\mathrm{P}$-Values for correlations are in parentheses. 
Table 2. Alerting Pearson Correlations of ERBP for each condition and EEG system (Brain Vision, BV, vs. Epoc Emotiv) using the IAPS Task.

\begin{tabular}{|c|c|c|c|c|c|c|c|c|c|}
\hline & $\begin{array}{c}\text { EEG } \\
\text { Frequency }\end{array}$ & $\begin{array}{c}\text { Epoc Low } \\
\text { Arousal } \\
\end{array}$ & $\begin{array}{c}\text { Epoc High } \\
\text { Arousal }\end{array}$ & $\begin{array}{l}\text { BV Low } \\
\text { Arousal }\end{array}$ & $\begin{array}{l}\text { BV High } \\
\text { Arousal }\end{array}$ & $\begin{array}{c}\text { Epoc Low } \\
\text { Valence }\end{array}$ & $\begin{array}{c}\text { Epoc High } \\
\text { Valence }\end{array}$ & $\begin{array}{l}\text { BV Low } \\
\text { Valence }\end{array}$ & $\begin{array}{l}\text { BV High } \\
\text { Valence }\end{array}$ \\
\hline $\begin{array}{c}\text { EEG } \\
\text { Frequency }\end{array}$ & 1 & & & & & & & & \\
\hline $\begin{array}{c}\text { Epoc Low } \\
\text { Arousal } \\
\end{array}$ & $\begin{array}{l}-.887^{*} \\
(0.045)\end{array}$ & 1 & & & & & & & \\
\hline $\begin{array}{c}\text { Epoc High } \\
\text { Arousal } \\
\end{array}$ & $\begin{array}{l}-.930^{*} \\
(0.022)\end{array}$ & $\begin{array}{l}.946^{*} \\
(0.015)\end{array}$ & 1 & & & & & & \\
\hline $\begin{array}{l}\text { BV Low } \\
\text { Arousal }\end{array}$ & $\begin{array}{l}0.658 \\
(0.227)\end{array}$ & $\begin{array}{l}-0.468 \\
(0.427)\end{array}$ & $\begin{array}{l}-0.586 \\
(0.299)\end{array}$ & 1 & & & & & \\
\hline $\begin{array}{l}\text { BV High } \\
\text { Arousal }\end{array}$ & $\begin{array}{l}0.666 \\
(0.219)\end{array}$ & $\begin{array}{l}-0.489 \\
(0.403)\end{array}$ & $\begin{array}{l}-0.624 \\
(0.260)\end{array}$ & $\begin{array}{l}.995^{* *} \\
(0.000)\end{array}$ & 1 & & & & \\
\hline $\begin{array}{c}\text { Epoc Low } \\
\text { Valence }\end{array}$ & $\begin{array}{l}-.881^{*} \\
(0.048)\end{array}$ & $\begin{array}{l}.992^{* *} \\
(0.001)\end{array}$ & $\begin{array}{l}.908^{*} \\
(0.033)\end{array}$ & $\begin{array}{l}-0.412 \\
(0.491)\end{array}$ & $\begin{array}{l}-0.423 \\
(0.478)\end{array}$ & 1 & & & \\
\hline $\begin{array}{c}\text { Epoc High } \\
\text { Valence }\end{array}$ & $\begin{array}{l}-.923^{*} \\
(0.025)\end{array}$ & $\begin{array}{l}.989^{* *} \\
(0.001)\end{array}$ & $\begin{array}{l}.983^{* *} \\
(0.003)\end{array}$ & $\begin{array}{l}-0.548 \\
(0.339)\end{array}$ & $\begin{array}{l}-0.575 \\
(0.310)\end{array}$ & $\begin{array}{l}.967^{* *} \\
(0.007)\end{array}$ & 1 & & \\
\hline $\begin{array}{l}\text { BV Low } \\
\text { Valence } \\
\end{array}$ & $\begin{array}{l}0.553 \\
(0.334)\end{array}$ & $\begin{array}{l}-0.297 \\
(0.627)\end{array}$ & $\begin{array}{l}-0.471 \\
(0.423)\end{array}$ & $\begin{array}{l}.975^{* *} \\
(0.005)\end{array}$ & $\begin{array}{l}.974^{* *} \\
(0.005)\end{array}$ & $\begin{array}{l}-0.231 \\
(0.708)\end{array}$ & $\begin{array}{l}-0.400 \\
(0.505)\end{array}$ & 1 & \\
\hline $\begin{array}{l}\text { BV High } \\
\text { Valence }\end{array}$ & $\begin{array}{l}0.523 \\
(0.366)\end{array}$ & $\begin{array}{l}-0.252 \\
(0.683)\end{array}$ & $\begin{array}{l}-0.449 \\
(0.448)\end{array}$ & $\begin{array}{l}.954^{*} \\
(0.012)\end{array}$ & $\begin{array}{l}.958^{*} \\
(0.010)\end{array}$ & $\begin{array}{l}-0.180 \\
(0.772)\end{array}$ & $\begin{array}{l}-0.364 \\
(0.548)\end{array}$ & $\begin{array}{l}.996^{* *} \\
(0.000)\end{array}$ & 1 \\
\hline
\end{tabular}

*. Correlation is significant at the 0.05 level (2-tailed).

$* *$. Correlation is significant at the 0.01 level (2-tailed).

${ }^{1}$ Exact $\mathrm{P}$-Values for correlations are in parentheses. 


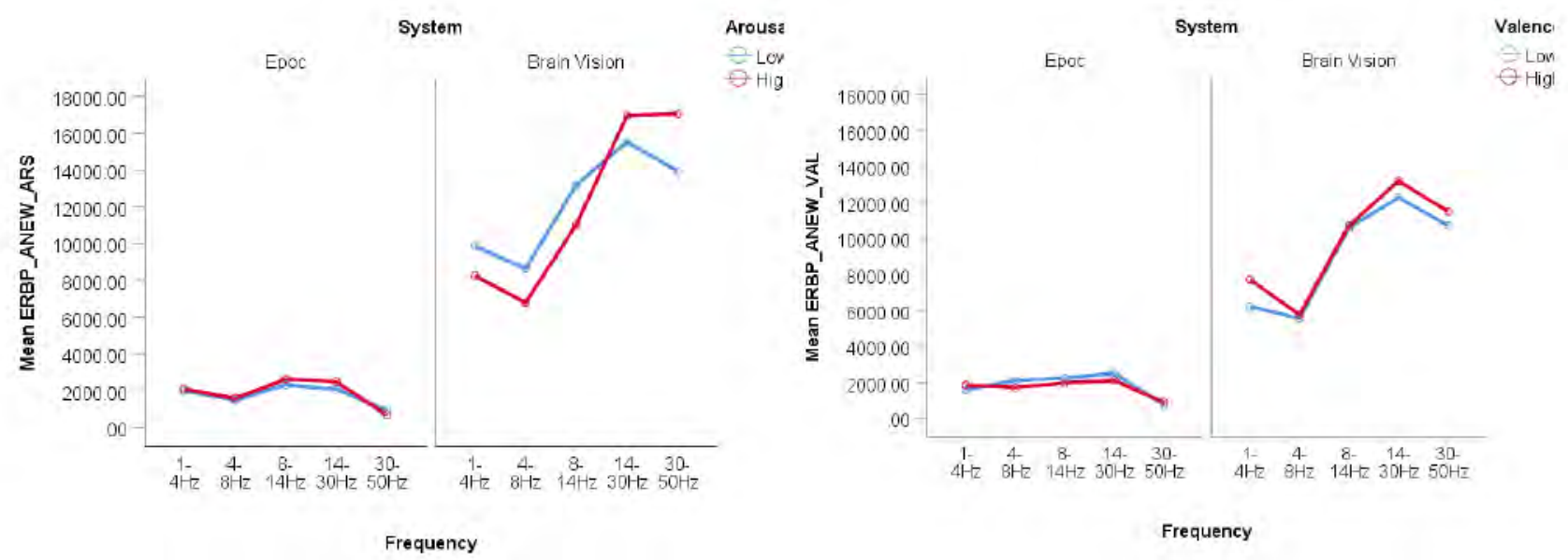

Figure 1. Distinguishing high and low arousal (Panel A) and valence (Panel B) between Epoc versus Brain Vision using event-related frequency band potentials (ERBP) with the ANEW task. $\mathrm{Y}$-axis values are in squared microvolts (EEG Power). From left to right, EEG bands in $\mathrm{x}$-axis correspond to standard nomenclature: delta, theta, alpha, beta and gamma.

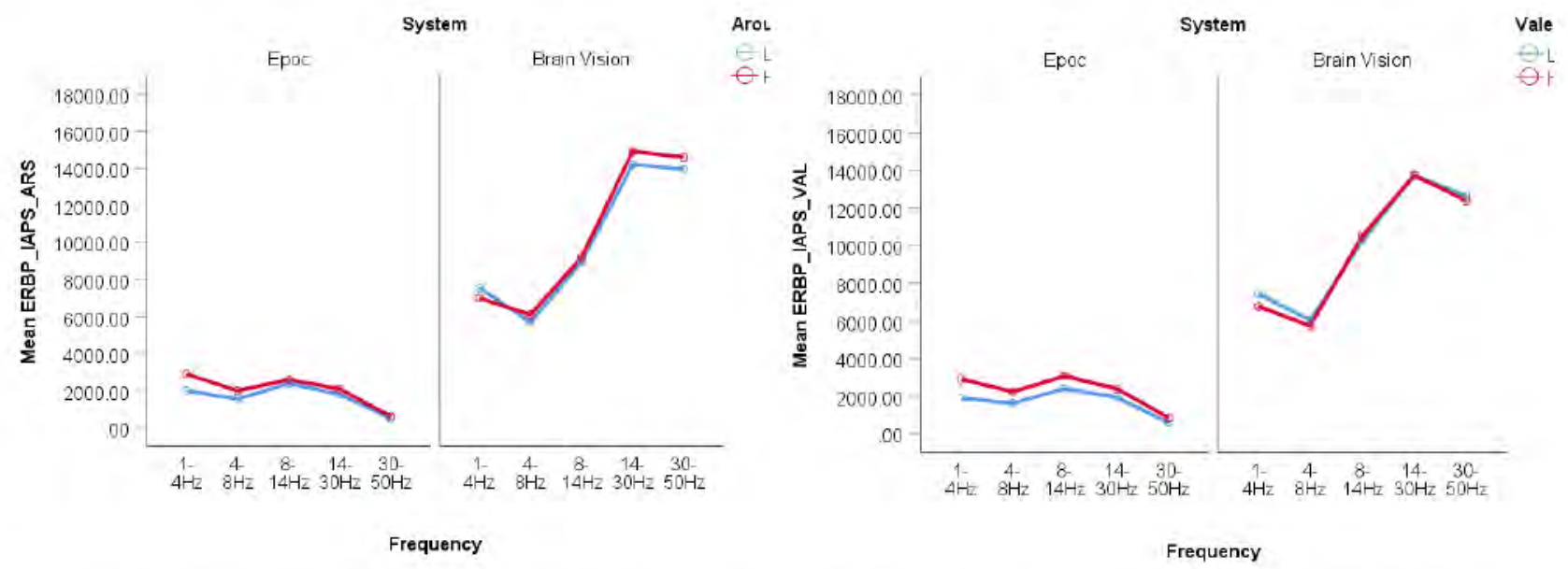

Figure 2. Distinguishing high and low arousal (Panel A) and valence (Panel B) between Epoc versus Brain Vision using event-related frequency band potentials (ERBP) with the IAPS task. $\mathrm{Y}$-axis values are in squared microvolts (EEG Power). From left to right, EEG bands in $\mathrm{x}$-axis correspond to standard nomenclature: delta, theta, alpha, beta and gamma. 